\title{
Applications of Pluripotential
}

\section{Theory to Algebraic Geometry}

\author{
Jean-Pierre Demailly \\ Université de Grenoble I, Institut Fourier
}

July 2011

\begin{abstract}
Plurisubharmonic functions and positive currents are an essential tool of modern complex analysis. Since their inception by Oka and Lelong in the mid 1940's, major applications to algebraic and analytic geometry have been developed in many directions. One of them is the Bochner-Kodaira technique, providing very strong existence theorems for sections of holomorphic vector bundles with positive curvature via $L^{2}$ estimates; one can mention here the foundational work achieved by Bochner, Kodaira, Nakano, Morrey, Kohn, Andreotti-Vesentini, Grauert, Hörmander, Bombieri, Skoda and OhsawaTakegoshi in the course of more than 4 decades. Another development is the theory of holomorphic Morse inequalities (1985), which relate certain curvature integrals with the asymptotic cohomology of large tensor powers of line or vector bundles, and bring a useful complement to the Riemann-Roch formula.

We describe here the main techniques involved in the proof of holomorphic Morse inequalities (chapter I) and their link with Monge-Ampère operators and intersection theory (chapter II). The last two chapters III, IV provide applications to the study of asymptotic cohomology functionals and the Green-Griffiths-Lang conjecture. The latter conjecture asserts that every entire curve drawn on a projective variety of general type should satisfy a global algebraic equation; via a probabilistic curvature calculation, holomorphic Morse inequalities imply that entire curves must at least satisfy a global algebraic differential equation.

The author expresses his warm thanks to the organizers of the CIME School in Pluripotential Theory held in Cetraro in July 2011, Filippo Bracci and John Erik Fornæss, for their invitation and the opportunity to deliver these lectures to an audience of young researchers. The author is also grateful to the referee for his (her) suggestions, and for a very careful reading of the manuscript.
\end{abstract}




\section{Contents}

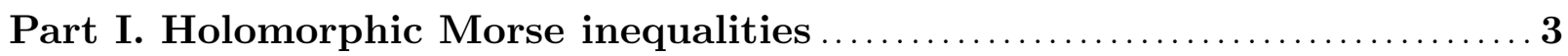

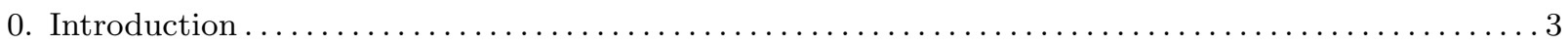

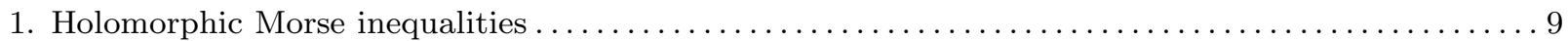

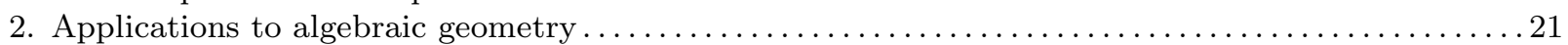

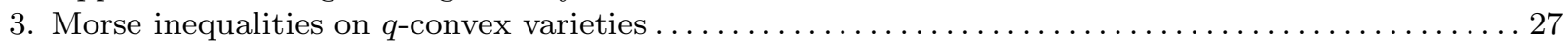

Part II. Approximation of currents and intersection theory ............. 31

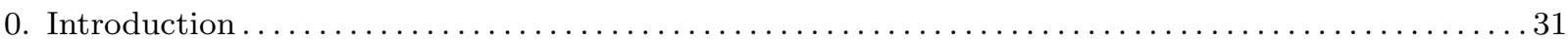

1. Pseudo-effective line bundles and singular Hermitian metrics $\ldots \ldots \ldots \ldots \ldots \ldots \ldots \ldots \ldots \ldots \ldots$

2. Hermitian metrics with minimal singularities and analytic Zariski decomposition . . . . . . . . . 33

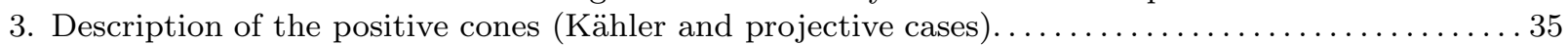

4. Approximation of plurisubharmonic functions via Bergman kernels $\ldots \ldots \ldots \ldots \ldots \ldots \ldots \ldots \ldots$

5. Global approximation of closed $(1,1)$-currents on a compact complex manifold $\ldots \ldots \ldots \ldots \ldots 43$

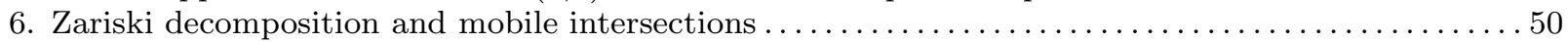

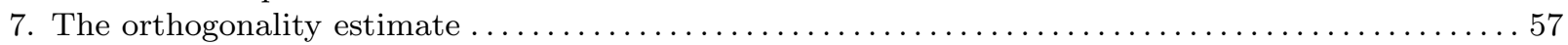

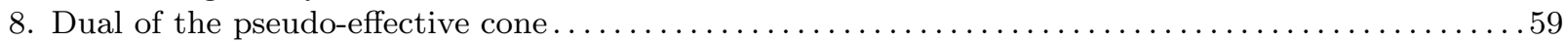

\section{Part III. Asymptotic cohomology functionals}

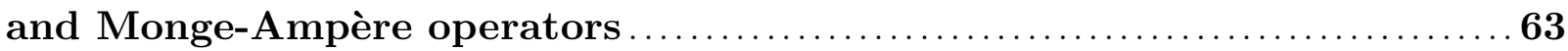

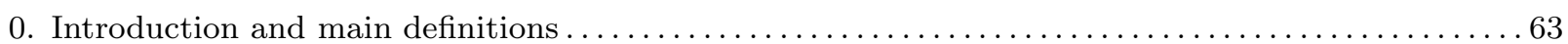

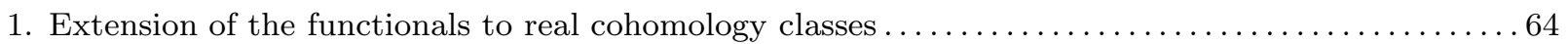

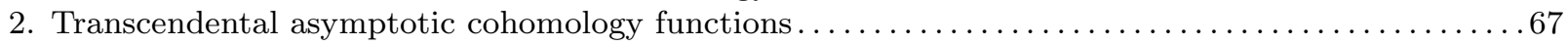

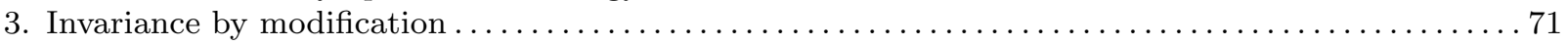

4. Proof of the infimum formula for the volume $\ldots \ldots \ldots \ldots \ldots \ldots \ldots \ldots \ldots \ldots \ldots \ldots \ldots \ldots \ldots \ldots$

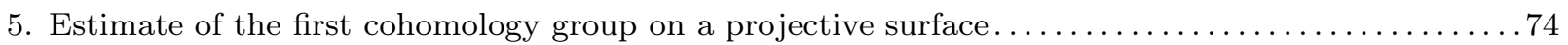

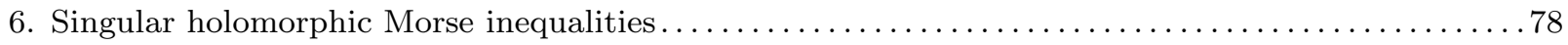

\section{Part IV. Morse inequalities and}

the Green-Griffiths-Lang conjecture $\ldots \ldots \ldots \ldots \ldots \ldots \ldots \ldots \ldots \ldots \ldots \ldots \ldots \ldots$

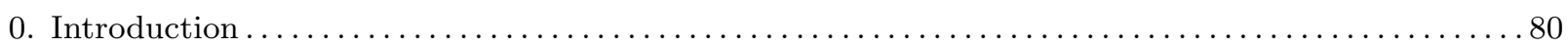

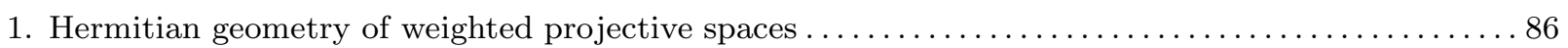

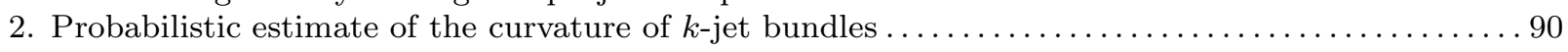

References $\ldots \ldots \ldots \ldots \ldots \ldots \ldots \ldots \ldots \ldots \ldots \ldots \ldots \ldots \ldots \ldots \ldots \ldots \ldots \ldots \ldots \ldots \ldots \ldots \ldots \ldots$ 


\section{Part I}

\section{Holomorphic Morse inequalities}

Holomorphic Morse inequalities provide asymptotic bounds for the cohomology of tensor powers of holomorphic line bundles. They are a very useful complement to the Riemann-Roch formula in many circumstances. They were first introduced in [Dem85], and were largely motivated by Siu's solution [Siu84, Siu85] of the Grauert-Riemenschneider conjecture, which we reprove here as a special case of a stronger statement. The basic tool is a spectral theorem which describes the eigenvalue distribution of complex Laplace-Beltrami operators. The original proof of [Dem85] was based partly on Siu's techniques and partly on an extension of Witten's analytic proof of standard Morse inequalities [Wit82]. Somewhat later Bismut [Bis87] and Getzler [Get89] gave new proofs, both relying on an analysis of the heat kernel in the spirit of the Atiyah-Bott-Patodi proof of the Atiyah-Singer index theorem [ABP73]. Although the basic idea is simple, Bismut used deep results arising from probability theory (the Malliavin calculus), while Getzler relied on his supersymmetric symbolic calculus for spin pseudodifferential operators [Get83].

We present here a slightly more elementary and self-contained proof which was suggested to us by Mohan Ramachadran on the occasion of a visit to Chicago in 1989. The reader is referred to [Dem85, Dem91] for more details.

\section{Introduction}

\section{A. Real Morse inequalities}

Let $M$ be a compact $C^{\infty}$ manifold, $\operatorname{dim}_{\mathbb{R}} M=m$, and $h$ a Morse function, i.e. a function such that all critical points are non degenerate. The standard (real) Morse inequalities relate the Betti numbers $b_{q}=\operatorname{dim} H_{D R}^{q}(M, \mathbb{R})$ and the numbers

$$
s_{q}=\# \text { critical points of index } q \text {, }
$$

where the index of a critical point is the number of negative eigenvalues of the Hessian form $\left(\partial^{2} h / \partial x_{i} \partial x_{j}\right)$. Specifically, the following "strong Morse inequalities" hold :

$$
b_{q}-b_{q-1}+\cdots+(-1)^{q} b_{0} \leqslant s_{q}-s_{q-1}+\cdots+(-1)^{q} s_{0}
$$

for each integer $q \geqslant 0$. As a consequence, one recovers the "weak Morse inequalities" $b_{q} \leqslant s_{q}$ and the expression of the Euler-Poincaré characteristic

$$
\chi(M)=b_{0}-b_{1}+\cdots+(-1)^{m} b_{m}=s_{0}-s_{1}+\cdots+(-1)^{m} s_{m} .
$$


These results are purely topological. They are obtained by showing that $M$ can be reconstructed from the structure of the Morse function by attaching cells according to the index of the critical points; real Morse inequalities are then obtained as a consequence of the Mayer-Vietoris exact sequence (see [Mil63]).

\section{B. Dolbeault cohomology}

Instead of looking at De Rham cohomology, we want to investigate here Dolbeault cohomology, i.e. cohomology of the $\bar{\partial}$-complex. Let $X$ be a compact complex manifold, $n=\operatorname{dim}_{\mathbb{C}} X$ and $E$ be a holomorphic vector bundle over $X$ with $\operatorname{rank} E=r$. Let us recall that there is a canonical $\bar{\partial}$-operator

$$
\bar{\partial}: C^{\infty}\left(X, \Lambda^{p, q} T_{X}^{*} \otimes E\right) \longrightarrow C^{\infty}\left(X, \Lambda^{p, q+1} T_{X}^{*} \otimes E\right)
$$

acting on spaces of $(p, q)$-forms with values in $E$. By the Dolbeault isomorphism theorem, there is an isomorphism

$$
H_{\bar{\partial}}^{p, q}(X, E):=H_{\frac{q}{\partial}}^{q}\left(C^{\infty}\left(X, \Lambda^{p, \bullet} T_{X}^{*} \otimes E\right)\right) \simeq H^{q}\left(X, \Omega_{X}^{p} \otimes \mathscr{G}(E)\right)
$$

from the cohomology of the $\bar{\partial}$-complex onto the cohomology of the sheaf of holomorphic $p$-forms with values in $E$. In particular, we have

$$
H_{\bar{\partial}}^{0, q}(X, E) \simeq H^{q}(X, \mathscr{O}(E)),
$$

and we will denote as usual $h^{q}(X, E)=\operatorname{dim} H^{q}(X, \mathscr{O}(E))$.

\section{C. Connections and curvature}

Leut us consider first a $C^{\infty}$ complex vector bundle $E \rightarrow M$ on a real differential manifold $M$ (without necessarily any holomorphic structure at this point). A connection $D$ on $E$ is a linear differential operator

$$
D: C^{\infty}\left(M, \Lambda^{q} T_{M}^{*} \otimes E\right) \rightarrow C^{\infty}\left(M, \Lambda^{q+1} T_{M}^{*} \otimes E\right)
$$

satisfying the Leibniz rule

$$
D(f \wedge s)=d f \wedge s+(-1)^{\operatorname{deg} f} f \wedge D s
$$

for all forms $f \in C^{\infty}\left(X, \Lambda^{p} T_{M}^{*}\right), s \in C^{\infty}\left(X, \Lambda^{q} T_{M}^{*} \otimes E\right)$. On an open set $U \subset M$ where $E$ is trivial, $E_{\mid U} \simeq U \times \mathbb{C}^{r}$, the Leibniz rule shows that a connection $D$ can be written in a unique way

$$
D s \simeq d s+\Gamma \wedge s
$$

where $\Gamma \in C^{\infty}\left(U, \Lambda^{1} T_{M}^{*} \otimes \operatorname{Hom}\left(\mathbb{C}^{r}, \mathbb{C}^{r}\right)\right)$ is an arbitrary $r \times r$ matrix of 1 -forms and $d$ acts componentwise. It is then easy to check that

$$
D^{2} s \simeq(d \Gamma+\Gamma \wedge \Gamma) \wedge s \quad \text { on } \quad U
$$

Therefore $D^{2} s=\theta_{D} \wedge s$ for some global 2-form $\theta_{D} \in C^{\infty}\left(M, \Lambda^{2} T_{M}^{*} \otimes \operatorname{Hom}(E, E)\right)$, given by $\theta_{D} \simeq d \Gamma_{U}+\Gamma_{U} \wedge \Gamma_{U}$ on any trivializing open set $U$ with a connection matrix $\Gamma_{U}$. 
(0.10) Definition. The (normalized) curvature tensor of $D$ is defined to be $\Theta_{D}=\frac{i}{2 \pi} \theta_{D}$, in other words

$$
\frac{i}{2 \pi} D^{2} s=\Theta_{D} \wedge s
$$

for any section $s \in C^{\infty}\left(M, \Lambda^{q} T_{M}^{*} \otimes E\right)$.

The main reason for the introduction of the factor $\frac{i}{2 \pi}$ is the well known formula for the expression of the Chern classes in the ring of differential forms of even degree: one has

$$
\operatorname{det}\left(\operatorname{Id}+\lambda \Theta_{D}\right)=1+\lambda \gamma_{1}(D)+\lambda^{2} \gamma_{2}(D)+\ldots+\lambda^{r} \gamma_{r}(D)
$$

where $\gamma_{j}(D)$ is a $d$-closed differential form of degree $2 j$. Moreover, $\gamma_{j}(D)$ has integral periods, i.e. the De Rham cohomology class $\left\{\gamma_{j}(D)\right\} \in H^{2 j}(M, \mathbb{R})$ is the image of an integral class, namely the $j$-th Chern class $c_{j}(E) \in H^{2 j}(M, \mathbb{Z})$.

\section{D. Hermitian connections}

Assume now that the fibers of $E$ are endowed with a $C^{\infty}$ Hermitian metric $h$, and that the isomorphism $E_{\mid U} \simeq U \times \mathbb{C}^{r}$ is given by a $C^{\infty}$ frame $\left(e_{\lambda}\right)$. Then we have a canonical sesquilinear pairing

$$
\begin{array}{ccc}
C^{\infty}\left(M, \Lambda^{p} T_{M}^{*} \otimes E\right) \times C^{\infty}\left(M, \Lambda^{q} T_{M}^{*} \otimes E\right) & \longrightarrow & C^{\infty}\left(M, \Lambda^{p+q} T_{M}^{*}\right) \\
(u, v) & \longmapsto & \{u, v\}_{h}
\end{array}
$$

given by

$$
\{u, v\}_{h}=\sum_{\lambda, \mu} u_{\lambda} \wedge \bar{v}_{\mu}\left\langle e_{\lambda}, e_{\mu}\right\rangle_{h} \quad \text { for } \quad u=\sum u_{\lambda} \otimes e_{\lambda}, \quad v=\sum v_{\mu} \otimes e_{\mu} .
$$

The connection $D$ is said to be Hermitian (or compatible with the Hermitian metric $h$ ) if it satisfies the additional property

$$
d\{u, v\}_{h}=\{D u, v\}_{h}+(-1)^{\operatorname{deg} u}\{u, D v\}_{h} .
$$

Assuming that $\left(e_{\lambda}\right)$ is $h$-orthonormal, one easily checks that $D$ is Hermitian if and only if the associated connection matrix $\Gamma$ is skew-symmetric, i.e. $\Gamma^{*}=-\Gamma$. In this case $\theta_{D}=d \Gamma+\Gamma \wedge \Gamma$ also satisfies $\theta_{D}^{*}=-\theta_{D}$, thus

$$
\Theta_{D}=\frac{i}{2 \pi} \theta_{D} \in C^{\infty}\left(M, \Lambda^{2} T_{M}^{*} \otimes \operatorname{Herm}(E, E)\right) .
$$

(0.13) Special case. For a bundle $E$ of rank $r=1$, the connection matrix $\Gamma$ of a Hermitian connection $D$ can be more conveniently written $\Gamma=-i A$ where $A$ is a real 1-form. Then we have

$$
\Theta_{D}=\frac{i}{2 \pi} d \Gamma=\frac{1}{2 \pi} d A
$$

Frequently, especially in physics, the real 2-form $B=d A=2 \pi \Theta_{D} \in C^{\infty}\left(M, \Lambda^{2} T_{M}^{*}\right)$ is referred to as the magnetic field, and the 1-form $A$ as its potential. A phase change $\tilde{s}(x)=s(x) e^{i \alpha(x)}$ in the isomorphism $E_{\mid U} \simeq U \times \mathbb{C}$ replaces $A$ with the new connection form $\tilde{A}=A+d \alpha$. 


\section{E. Connections on a Hermitian holomorphic vector bundle}

If $M=X$ is a complex manifold, every connection $D$ can be split in a unique way as the sum $D=D^{\prime}+D^{\prime \prime}$ of a $(1,0)$-connection $D^{\prime}$ and a $(0,1)$-connection $D^{\prime \prime}$ :

$$
\begin{aligned}
D^{\prime} & : C^{\infty}\left(M, \Lambda^{p, q} T_{X}^{*} \otimes E\right) \longrightarrow C^{\infty}\left(M, \Lambda^{p+1, q} T_{X}^{*} \otimes E\right), \\
D^{\prime \prime}: C^{\infty}\left(M, \Lambda^{p, q} T_{X}^{*} \otimes E\right) & \longrightarrow C^{\infty}\left(M, \Lambda^{p, q+1} T_{X}^{*} \otimes E\right) .
\end{aligned}
$$

In a local trivialization given by a $C^{\infty}$ frame, one can write

$$
\begin{aligned}
D^{\prime} u & =d^{\prime} u+\Gamma^{\prime} \wedge u \\
D^{\prime \prime} u & =d^{\prime \prime} u+\Gamma^{\prime \prime} \wedge u
\end{aligned}
$$

with $\Gamma=\Gamma^{\prime}+\Gamma^{\prime \prime}$ and $d^{\prime}=\partial, d^{\prime \prime}=\bar{\partial}$. If $(E, h)$ is a $C^{\infty}$ Hermitian structure, the connection is Hermitian if and only if $\Gamma^{\prime}=-\left(\Gamma^{\prime \prime}\right)^{*}$ in any $h$-orthonormal frame. Thus there exists a unique Hermitian connection corresponding to a prescribed $(0,1)$ part $D^{\prime \prime}$.

Assume now that the Hermitian bundle $(E, h)$ has a holomorphic structure. The unique Hermitian connection $D$ for which $D^{\prime \prime}=\bar{\partial}$ is called the Chern connection of $(E, h)$. In a local holomorphic frame $\left(e_{\lambda}\right)$ of $E_{\mid U}$, the metric $h$ is given by some Hermitian matrix $H=\left(h_{\lambda \mu}\right)$ where $h_{\lambda \mu}=\left\langle e_{\lambda}, e_{\mu}\right\rangle_{h}$. Standard computations yield the expression of the Chern connection :

$$
\left\{\begin{aligned}
D^{\prime} s & =\partial s+\bar{H}^{-1} \partial \bar{H} \wedge s \\
D^{\prime \prime} s & =\bar{\partial} s \\
\theta_{D} \wedge s & =D^{2} s=\left(D^{\prime} D^{\prime \prime}+D^{\prime \prime} D^{\prime}\right) s=-\bar{\partial}\left(\bar{H}^{-1} \partial \bar{H}\right) \wedge s
\end{aligned}\right.
$$

(0.14) Definition. The Chern curvature tensor of $(E, h)$ is the curvature tensor of its Chern connection, denoted

$$
\theta_{E, h}=D^{\prime} D^{\prime \prime}+D^{\prime \prime} D^{\prime}=-\bar{\partial}\left(\bar{H}^{-1} \partial \bar{H}\right)
$$

In the special case of a rank 1 bundle $E$, the matrix $H$ is simply a positive function, and it is convenient to introduce its weight $\varphi$ such that $H=\left(e^{-\varphi}\right)$ where $\varphi \in C^{\infty}(U, \mathbb{R})$ depends on the given trivialization $E_{\mid U} \simeq U \times \mathbb{C}$. We have in this case

$$
\Theta_{E, h}=\frac{i}{2 \pi} \theta_{E, h}=\frac{i}{2 \pi} \partial \bar{\partial} \varphi \quad \text { on } \quad U
$$

and therefore $\Theta_{E, h}$ is a closed real $(1,1)$-form.

\section{F. Fundamental facts of Hodge theory}

Assume here that $M$ is a Riemannian manifold with metric $g=\sum g_{i j} d x_{i} \otimes d x_{j}$. Given $q$-forms $u, v$ on $M$ with values in $E$, we consider the global $L^{2}$ norm and inner product

$$
\|u\|^{2}=\int_{M}|u(x)|^{2} d \sigma(x), \quad\langle\langle u, v\rangle\rangle=\int_{M}\langle u(x), v(x)\rangle d \sigma(x),
$$


where $|u|$ is the pointwise Hermitian norm and $d \sigma$ the Riemannian volume form. The Laplace Beltrami operator associated with the connection $D$ is

$$
\Delta=D D^{*}+D^{*} D
$$

acting on any of the spaces $C^{\infty}\left(M, \Lambda^{q} T_{M}^{*} \otimes E\right)$; here

$$
D^{*}: C^{\infty}\left(M, \Lambda^{q} T_{M}^{*} \otimes E\right) \longrightarrow C^{\infty}\left(M, \Lambda^{q-1} T_{M}^{*} \otimes E\right)
$$

is the (formal) $L^{2}$ adjoint of $D$. The complex Laplace operators $\Delta^{\prime}=D^{\prime} D^{\prime *}+D^{\prime *} D^{\prime}$ and $\Delta^{\prime \prime}=D^{\prime \prime} D^{\prime \prime *}+D^{\prime \prime *} D^{\prime \prime}$ are defined similarly when $M=X$ is a complex manifold. In degree 0 we simply have $\Delta=D^{*} D$. A well-known calculation shows that the principal symbol of $\Delta$ is $\sigma_{\Delta}(x, \xi)=-|\xi|^{2}$ Id (while $\sigma_{\Delta^{\prime}}(x, \xi)=\sigma_{\Delta^{\prime \prime}}(x, \xi)=-\frac{1}{2}|\xi|^{2}$ Id). As a consequence $\Delta, \Delta^{\prime}, \Delta^{\prime \prime}$ are always elliptic operators.

When $M$ is compact, the operator $\Delta$ acting on any of the spaces $C^{\infty}\left(M, \Lambda^{q} T_{M}^{*} \otimes E\right)$ has a discrete spectrum

$$
\lambda_{1} \leqslant \lambda_{2} \leqslant \cdots \leqslant \lambda_{j} \leqslant \cdots
$$

and corresponding eigenfunctions $\psi_{j} \in C^{\infty}\left(M, \Lambda^{q} T_{M}^{*} \otimes E\right)$, depending of course on $q$.

Our main goal is to obtain asymptotic formulas for the eigenvalues. For this, we will make an essential use of the heat operator $e^{-t \Delta}$. In the above setting, the heat operator is the bounded Hermitian operator associated to the heat kernel

$$
K_{t}(x, y)=\sum_{\nu=1}^{+\infty} e^{-\lambda_{\nu} t} \psi_{\nu}(x) \otimes \psi_{\nu}^{*}(y),
$$

i.e.

$$
\left\langle\left\langle u, e^{-t \Delta} v\right\rangle\right\rangle=\int_{M \times M}\left\langle u(x), K_{t}(x, y) \cdot v(y)\right\rangle d \sigma(x) d \sigma(y) .
$$

Standard results of the theory of elliptic operators show that

$$
K_{t} \in C^{\infty}(] 0,+\infty[\times M \times M, \operatorname{Hom}(E, E))
$$

and that $K_{t}(x, y)$ is the solution of the differential equation

$$
\frac{\partial}{\partial t} K_{t}(x, y)=-\Delta_{x} K_{t}(x, y), \quad \lim _{t \rightarrow 0_{+}} K_{t}(x, y)=\delta_{y}(x) \quad(\text { Dirac at } y)
$$

as follows formally from the fact that $\frac{\partial}{\partial t} e^{-t \Delta}=-\Delta e^{-t \Delta}$ and $e^{-0 \Delta}=\mathrm{Id}$. The asymptotic distribution of eigenvalues can be recovered from the straightforward formula

$$
\sum_{\nu=1}^{+\infty} e^{-\lambda_{\nu} t}=\int_{M} \operatorname{tr}_{E} K_{t}(x, x) d \sigma(x) .
$$

In the sequel, we are especially interested in the 0-eigenspace:

(0.21) Definition. The space of $\Delta$-harmonic forms is defined to be

$$
\mathscr{H}_{\Delta}^{q}(M, E)=\operatorname{Ker} \Delta=\left\{u \in C^{\infty}\left(M, \Lambda^{q} T_{M}^{*} \otimes E\right) ; \Delta u=0\right\} .
$$


When $M$ is compact, an integration by part shows that

$$
\langle\langle\Delta u, u\rangle\rangle=\|D u\|^{2}+\left\|D^{*} u\right\|^{2},
$$

hence $u$ is $\Delta$-harmonic if and only if $D u=D^{*} u=0$. Moreover, as $\Delta$ is a self-ajoint operator, standard elliptic theory implies that

$$
C^{\infty}\left(M, \Lambda^{q} T_{M}^{*} \otimes E\right)=\operatorname{Ker} \Delta \oplus \operatorname{Im} \Delta=\mathscr{H}_{\Delta}^{q}(M, E) \oplus \operatorname{Im} \Delta,
$$

and $\operatorname{Ker} \Delta=\mathscr{H}_{\Delta}^{q}(M, E), \operatorname{Im} \Delta$ are orthogonal with respect to the $L^{2}$ inner product. Clearly $\operatorname{Im} \Delta \subset \operatorname{Im} D+\operatorname{Im} D^{*}$, and both images $\operatorname{Im} D, \operatorname{Im} D^{*}$ are orthogonal to the space of harmonic forms by what we have just seen. As a consequence, we have

$$
\operatorname{Im} \Delta=\operatorname{Im} D+\operatorname{Im} D^{*} .
$$

(0.24) Hodge isomorphism theorem. Assume that $M$ is compact and that $D$ is an integrable connection, i.e. $D^{2}=0$ ( or $\theta_{D}=0$ ). Then $D$ defines on spaces of sections $C^{\infty}\left(M, \Lambda^{q} T_{M}^{*} \otimes E\right)$ a differential complex which can be seen as a generalization of the De Rham complex. The condition $D^{2}=0$ immediately implies that $\operatorname{Im} D \perp \operatorname{Im} D^{*}$ and we conclude from the above discussion that there is an orthogonal direct sum

$$
C^{\infty}\left(M, \Lambda^{q} T_{M}^{*} \otimes E\right)=\mathscr{H}_{\Delta}^{q}(M, E) \oplus \operatorname{Im} D \oplus \operatorname{Im} D^{*} .
$$

If we put $u=h+D v+D^{*} w$ according to this decomposition, then $D u=D D^{*} w=0$ if and only if $\left\|D^{*} w\right\|=\left\langle\left\langle D D^{*} w, w\right\rangle\right\rangle=0$, thus

$$
\operatorname{Ker} D=\mathscr{H}_{\Delta}^{q}(M, E) \oplus \operatorname{Im} D .
$$

This implies the Hodge isomorphism theorem

$$
H_{\mathrm{DR}}^{q}(M, E):=\operatorname{Ker} D / \operatorname{Im} D \simeq \mathscr{H}_{\Delta}^{q}(M, E) .
$$

In case $M=X$ is a compact complex manifold, $(E, h)$ a Hermitian holomorphic vector bundle and $D=D^{\prime}+D^{\prime \prime}$ the Chern connection, the integrability condition $D^{\prime \prime 2}=\bar{\partial}^{2}=0$ is always satisfied. Thus we get an analogous isomorphism

$$
H^{q}(X, \mathscr{G}(E)) \simeq H_{\bar{\partial}}^{0, q}(X, E) \simeq \mathscr{H}_{\Delta^{\prime \prime}}^{0, q}(M, E),
$$

and more generally

$$
H^{q}\left(X, \Omega_{X}^{p} \otimes \mathscr{G}(E)\right) \simeq H_{\frac{\partial}{\partial}}^{p, q}(X, E) \simeq \mathscr{H}_{\Delta^{\prime \prime}}^{p, q}(M, E),
$$

where $\mathscr{H}_{\Delta^{\prime \prime}}^{p, q}(M, E)$ is the space of $\Delta^{\prime \prime}$-harmonic forms of type $(p, q)$ with values in $E$.

(0.28) Corollary (Hodge decomposition theorem). If $(X, \omega)$ is a compact Kähler manifold and $(E, h)$ is a flat Hermitian vector bundle over $X$ (i.e. $\left.D_{E, h}^{2}=0\right)$, then there is an isomorphism

$$
H_{\mathrm{DR}}^{k}(M, E) \simeq \bigoplus_{p+q=k} H_{\frac{p}{\partial}, q}(X, E)
$$

In fact, under the condition that $\omega$ is Kähler, i.e. $d \omega=0$, well-known identities of Kähler geometry imply $\Delta^{\prime}=\Delta^{\prime \prime}=\frac{1}{2} \Delta$, and as a consequence

$$
\mathscr{H}_{\Delta}^{k}(M, E)=\bigoplus_{p+q=k} \mathscr{H}_{\Delta^{\prime \prime}}^{p, q}(X, E) .
$$




\section{Holomorphic Morse inequalities}

\section{A. Main statements}

Let $X$ be a compact complex $n$-dimensional manifold, $L \rightarrow X$ a holomorphic line bundle and $E \rightarrow X$ a holomorphic vector bundle of rank $r=\operatorname{rank} E$. We assume that $L$ is equipped with a smooth Hermitian metric $h$ and denote accordingly $\Theta_{L, h}$ its curvature form; by definition this is a closed real $(1,1)$-form and its cohomology class $c_{1}(L)_{\mathbb{R}}=\left\{\Theta_{L, h}\right\} \in H_{\mathrm{DR}}^{2}(X, \mathbb{R})$ is the first Chern class of $L$.

(1.1) q-index sets. We define the $q$-index sets and $\{\leqslant q\}$-index sets of $(L, h)$ to be

$$
\begin{aligned}
X(L, h, q) & =\left\{x \in X ; \Theta_{L, h}(x) \text { has } \begin{array}{cr}
q & \text { negative eigenvalues } \\
n-q & \text { positive eigenvalues }
\end{array}\right\} \\
X(L, h, \leqslant q) & =\bigcup_{1 \leqslant j \leqslant q} X(L, h, j) .
\end{aligned}
$$

Clearly $X(L, h, q)$ and $X(L, h, \leqslant q)$ are open subsets of $X$, and we have a partition into "chambers" $X=S \cup \bigcup_{0 \leqslant q \leqslant n} X(L, h, q)$ where $S=\left\{x \in X ; \Theta_{L, h}(x)=0\right\}$ is the degeneration set. The following theorem was first proved in [Dem85].

(1.2) Main Theorem. The cohomology groups of tensor powers $E \otimes L^{k}$ satisfy the following asymptotic estimates as $k \rightarrow+\infty$ :

(1.2) $\mathrm{WM}$ Weak Morse inequalities:

$$
h^{q}\left(X, E \otimes L^{k}\right) \leqslant r \frac{k^{n}}{n !} \int_{X(L, h, q)}(-1)^{q} \Theta_{L, h}^{n}+o\left(k^{n}\right) .
$$

$(1.2)_{\mathrm{SM}}$ Strong Morse inequalities:

$$
\sum_{0 \leqslant j \leqslant q}(-1)^{q-j} h^{j}\left(X, E \otimes L^{k}\right) \leqslant r \frac{k^{n}}{n !} \int_{X(L, h, \leqslant q)}(-1)^{q} \Theta_{L, h}^{n}+o\left(k^{n}\right) .
$$

$(1.2)_{\mathrm{RR}}$ Asymptotic Riemann-Roch formula:

$$
\chi\left(X, E \otimes L^{k}\right):=\sum_{0 \leqslant j \leqslant n}(-1)^{j} h^{j}\left(X, E \otimes L^{k}\right)=r \frac{k^{n}}{n !} \int_{X} \Theta_{L, h}^{n}+o\left(k^{n}\right) .
$$

The weak Morse form (1.2) $)_{\mathrm{WM}}$ follows from strong Morse (1.2) $\mathrm{SM}$ by adding consecutive inequalities for the indices $q-1$ and $q$, since the signs $(-1)^{q-j}$ and $(-1)^{q-1-j}$ are opposite. Also, $(1.2)_{\mathrm{RR}}$ is just a weaker formulation of the existence of the Hilbert polynomial, and as such, is a consequence of the Hirzebruch-Riemann-Roch formula; it follows formally from $(1.2)_{\mathrm{SM}}$ with $q=n$ and $q=n+1$, since $h^{n+1}=0$ identically and the signs are reversed. Now, by adding $(1.2)_{\mathrm{SM}}$ for the indices of opposite parity $q+1$ and $q-2$, we find

$$
h^{q+1}\left(X, E \otimes L^{k}\right)-h^{q}(\ldots)+h^{q-1}(\ldots) \leqslant r \frac{k^{n}}{n !} \int_{X(L, h,\{q-1, q, q+1\})}(-1)^{q+1} \Theta_{L, h}^{n}+o\left(k^{n}\right),
$$


where $X(L, h,\{q-1, q, q+1\})$ is meant for the union of chambers of indices $q-1, q$, $q+1$. As a consequence, we get lower bounds for the cohomology groups:

$$
h^{q}\left(X, E \otimes L^{k}\right) \geqslant h^{q}-h^{q+1}-h^{q-1} \geqslant r \frac{k^{n}}{n !} \int_{X(L, h,\{q-1, q, q+1\})}(-1)^{q} \Theta_{L, h}^{n}-o\left(k^{n}\right) .
$$

Another important special case is $(1.2)_{\mathrm{SM}}$ for $q=1$, which yields the lower bound

$$
h^{0}\left(X, E \otimes L^{k}\right) \geqslant h^{0}-h^{1} \geqslant r \frac{k^{n}}{n !} \int_{X(L, h, \leqslant 1)} \Theta_{L, h}^{n}-o\left(k^{n}\right) .
$$

As we will see later in the applications, this lower bound provides a very useful criterion to prove the existence of sections of large tensor powers of a line bundle.

\section{B. Heat kernel and eigenvalue distribution}

We introduce here a basic heat equation technique, from which all asymptotic eigenvalue estimates can be derived via an explicit formula, known as Mehler's formula.

We start with a compact Riemannian manifold $(M, g)$ with $\operatorname{dim}_{\mathbb{R}} M=m$, and denote by $d \sigma$ its Riemannian volume form. Let $\left(L, h_{L}\right)\left(\operatorname{resp} .\left(E, h_{E}\right)\right)$ be a Hermitian complex line (resp. vector bundle) on $M$, equipped with a Hermitian connection $D_{L}$ (resp. $\left.D_{E}\right)$.

We denote by $D_{k}=D_{E \otimes L^{k}}$ the associated connection on $E \otimes L^{k}$, and by $\Delta_{k}=D_{k}^{*} D_{k}$ the Laplace-Beltrami operator acting on sections of $E \otimes L^{k}$ (i.e. forms of degree 0 ). As in (0.13), we introduce the (local) connection form $\Gamma_{L}=-i A$ of $L$ and the corresponding (global) curvature 2 -form $B=d A \in C^{\infty}\left(M, \Lambda^{2} T_{M}^{*}\right)$, i.e. the "magnetic field" ( $\Gamma_{E}$ and the corresponding curvature tensor $\Theta_{E}$ of $D_{E}$ will not play a significant role here). Finally, we assume that an additional section $V \in C^{\infty}(M, \operatorname{Herm}(E, E))$ is given ("electric field") ; for simplicity of notation, we still denote by $V$ the operator $V \otimes \operatorname{Id}_{L^{k}}$ acting on $E \otimes L^{k}$.

If $\Omega \subset M$ is a smoothly bounded open subset of $M$, we consider for $u$ in the Sobolev space $W_{0}^{1}\left(\Omega, E \otimes L^{k}\right)$ the quadratic form

$$
Q_{k, \Omega}(u)=\int_{\Omega} \frac{1}{k}\left|D_{k} u\right|^{2}-\langle V u, u\rangle .
$$

Here $W_{0}^{1}\left(\Omega, E \otimes L^{k}\right)$ is the closure of the space of smooth sections with compact support in $\Omega$, taken in the Hilbert space $W_{\text {loc }}^{1}\left(M, E \otimes L^{k}\right)$ of sections that have $L_{\text {loc }}^{2}$ coefficients as well as their first derivatives. In other words, we consider the densily defined self adjoint operator

$$
\square_{k}=\frac{1}{k} D_{k}^{*} D_{k}-V
$$

acting in the Hilbert space $W_{0}^{1}\left(\Omega, E \otimes L^{k}\right)$, i.e. with Dirichlet boundary conditions. Again, $\square_{k}$ acting on $W_{0}^{1}\left(\Omega, E \otimes L^{k}\right)$ has a discrete spectrum whenever $\Omega$ is relatively compact (and also sometimes when $\Omega$ is unbounded, according to the behavior of $B$ and $V$ at infinity; except otherwise stated, we will assume that we are in this case later on). Then, there is an associated "localized" heat kernel

$$
K_{t, k, \Omega}(x, y)=\sum_{\nu=1}^{+\infty} e^{-\lambda_{\nu, k, \Omega} t} \psi_{\nu, k, \Omega}(x) \otimes \psi_{\nu, k, \Omega}^{*}(y)
$$


where $\psi_{\nu, k, \Omega} \in W_{0}^{1}\left(\Omega, E \otimes L^{k}\right)$ are the eigenfunctions and $\lambda_{\nu, k, \Omega}$ their eigenvalues.

We want to study the asymptotic eigenvalue distribution of $\square_{k}$ as $k \rightarrow+\infty$, and more precisely get an asymptotic formula for the corresponding heat kernel $e^{-t \square_{k}}$. The basic idea is to decompose the proof in three steps :

$(\alpha)$ convince ourselves that the asymptotic estimates can be "localized", up to lower order error terms.

$(\beta)$ show that the local estimates can be obtained by freezing the coefficients of the operators involved at any given point.

$(\gamma)$ compute explicitly the heat kernel in the case of connections with constant curvature, assuming moreover that $\Omega \simeq \mathbb{R}^{m}$ with the flat euclidean metric.

$(\alpha)$ In order to see that the situation can be localized, we fix a partition of unity $\left(\tau_{j}\right)$ relative to an arbitrarily fine finite covering $\left(\Omega_{j}\right)$ of $\bar{\Omega}$, such that $\sum \tau_{j}^{2}=1$ near $\bar{\Omega}$. We consider the continuous injection

$$
I_{\Omega, \Omega_{j}}: W_{0}^{1}\left(\Omega, E \otimes L^{k}\right) \rightarrow \bigoplus_{j} W_{0}^{1}\left(\Omega \cap \Omega_{j}, E \otimes L^{k}\right), \quad u \mapsto\left(\tau_{j} u\right)_{j}
$$

the inverse of which is $\left(u_{j}\right) \mapsto u=\sum \tau_{j} u_{j}$. As $\sum \tau_{j} d \tau_{j}=0$ on $\Omega$, we find

$$
\sum_{j} Q_{k, \Omega_{j}}\left(\tau_{j} u\right)-Q_{k, \Omega}(u)=\frac{1}{k} \int_{\Omega}\left(\sum\left|d \tau_{j}\right|^{2}\right)|u|^{2} \leqslant O\left(\frac{1}{k}\right)|u|^{2}
$$

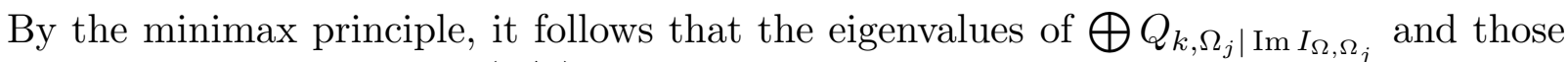
of $Q_{k, \Omega}$ differ by at most $O(1 / k)$ as $k \rightarrow+\infty$. This explains why a localization process is possible, at least as far as the eigenvalue distribution is concerned. For the related heat kernels on small geodesic balls, one can use the following localization principle.

(1.9) Proposition. Let $\Omega_{\rho}=B\left(x^{0}, \rho\right)$ be a geodesic ball of $(M, g)$ of radius $\rho$ where $\rho<$ injectivity radius. Then there exist constants $C_{1}$ and $\varepsilon_{1}>0$ such that for all $\left.t \in] 0, \min \left(k \varepsilon_{1}, k \rho^{2} / 2 m\right)\right]$ and every $x_{0} \in M$ we have

$$
\left|K_{t, k, M}\left(x^{0}, x^{0}\right)-K_{t, k, \Omega_{\rho}}\left(x^{0}, x^{0}\right)\right| \leqslant C_{1}\left(\frac{k}{t}\right)^{m / 2} \exp \left(-\frac{k \rho^{2}}{4 t}+2 t \sup _{\Omega_{\rho}}\|V\|\right) .
$$

A proof of this technical result is given in Thierry Bouche's PhD thesis (cf. [Bou90]). It relies on a use of Kato's inequality (cf. [HeSU80]), which amounts to say that we get an upper bound for $K_{t, k, M}$ in the case when the curvature is trivial; one can then use the calculations given below to get the explicit bound, see e.g. $\left(1.10^{\prime}\right)$.

$(\beta)$ Now, let $x^{0} \in M$ be a given point. We choose coordinates $\left(x_{1}, \ldots, x_{m}\right)$ centered at $x^{0}$ such that $\left(\partial / \partial x_{1}, \ldots, \partial / \partial x_{m}\right)$ is orthonormal at $x^{0}$ with respect to the Riemannian metric $g$. By changing the orthonormal frame of $L$ as in (0.13), we can adjust the connection form $\Gamma_{L}=-i A$ of $L$ to be given by any local potential $A(x)=\sum_{j} A_{j}(x) d x_{j}$ such that $B=d A$, and we can therefore arrange that $A\left(x^{0}\right)=0$. Similarly, we can fix 
a unitary frame of $E$ such that $\Gamma_{E}\left(x^{0}\right)=0$. Set $x^{0}=0$ for simplicity. The first term of our Laplace operator $\square_{k}=\frac{1}{k} D_{k}^{*} D_{k}-V$ is the square of the first order operator

$$
\begin{aligned}
k^{-1 / 2} D_{k} u(x) & =k^{-1 / 2}\left(d u(x)+k \operatorname{Id}_{E} \otimes \Gamma_{L}(x) \cdot u(x)+\operatorname{Id}_{L^{k}} \otimes \Gamma_{E}(x) \cdot u(x)\right) \\
& =k^{-1 / 2} \sum_{j}\left(\frac{\partial u}{\partial x_{j}}-i k^{1 / 2} A_{j}(x) u(x)\right) d x_{j}+k^{-1 / 2} \operatorname{Id}_{L^{k}} \otimes \Gamma_{E}(x) \cdot u(x) .
\end{aligned}
$$

If we use a rescaling $x=k^{-1 / 2} \widetilde{x}$ and set $\widetilde{u}(\widetilde{x})=u(x)=u\left(k^{-1 / 2} \widetilde{x}\right)$, this operator takes the form

$$
\widetilde{D}_{k} \widetilde{u}(\widetilde{x})=\sum_{j}\left(\frac{\partial \widetilde{u}}{\partial \widetilde{x}_{j}}-i k^{1 / 2} A_{j}\left(k^{-1 / 2} \widetilde{x}\right) \widetilde{u}(\widetilde{x})\right) d x_{j}+O\left(k^{-1 / 2}|\widetilde{x}|\right) \widetilde{u}(\widetilde{x}) d x .
$$

As $A_{j}(0)=0$, the term $k^{1 / 2} A_{j}\left(k^{-1 / 2} \widetilde{x}\right)$ converges modulo $O\left(k^{-1 / 2}|\widetilde{x}|^{2}\right)$ terms to the linearized part $\widetilde{A}_{j}(\widetilde{x})=\sum_{i, j} \frac{\partial A_{j}}{\partial x_{i}}(0) \widetilde{x}_{i}$. Observe also that the connection form $\Gamma_{E}$ of $E$ only contributes for terms of the form $O\left(k^{-1 / 2}|\widetilde{x}|\right.$ ) (and thus will be negligible in the end, together with the quadratic terms of $A_{j}$ ). Our initial operator $\square_{k}=\frac{1}{k} D_{k}^{*} D_{k}-V$ becomes

$$
\widetilde{\square}_{k}=\widetilde{D}_{k}^{*} \widetilde{D}_{k}-\widetilde{V}
$$

where $\widetilde{V}(\widetilde{x})=V\left(k^{-1 / 2} \widetilde{x}\right)$ and where the ajoint is computed with respect to the rescaled metric $\widetilde{g}(x)=\sum g_{i j}\left(k^{-1 / 2} \widetilde{x}\right) d \widetilde{x}_{j} d \widetilde{x}_{j}$; here $\widetilde{g} \rightarrow \sum\left(d \widetilde{x}_{j}\right)^{2}$ as $k \rightarrow+\infty$ thanks to the assumption that $g_{i j}(0)=\delta_{i j}$. Modulo lower order terms $O\left(k^{-1 / 2}|\widetilde{x}|^{2}\right), \widetilde{D}_{k}$ is given by a linear connection form

$$
\widetilde{A}(\widetilde{x})=\sum B_{i j} \widetilde{x}_{i} d \widetilde{x}_{j}
$$

assciated with the constant magnetic field $B\left(x^{0}\right)=\sum_{i, j} B_{i j} d x_{i} \wedge d x_{j}$ frozen at $x^{0}=0$. We can moreover choose orthonormal coordinates so that $B\left(x^{0}\right)$ takes the standard form

$$
B\left(x^{0}\right)=\sum_{j=1}^{s} B_{j} d x_{j} \wedge d x_{j+s}
$$

where $2 s \leqslant m$ is the rank of the alternate 2 -form $B\left(x^{0}\right)$ and $B_{j}$ the curvature eigenvalues with respect to $g\left(x^{0}\right)$. The corresponding linearized potential is

$$
\widetilde{A}(\widetilde{x})=\sum_{j=1}^{s} B_{j} \widetilde{x}_{j} d \widetilde{x}_{j+s}
$$

The intuition from Physics is that the eigenfunctions represent "waves" of heat propagation of a certain typical wave length $\lambda$ in the coordinates $\widetilde{x}$, and of a corresponding (much shorter) wave length $\lambda k^{-1 / 2}$ in the original coordinates. At that scale, our space behaves as if the metrics were flat and the curvature constant.

$(\gamma)$ Let us consider the operators obtained by "freezing" the coefficients at any point $x^{0}$, as explained at step $(\beta)$, although we will not perform the rescaling here. More specifically, we assume that 
- $\quad L$ has constant curvature $B=\sum_{j=1}^{s} B_{j} d x_{j} \wedge d x_{j+s}$. Then there is a local trivialization in which

$$
D_{L} u=d u-i A \wedge u, \quad A=\sum_{j=1}^{s} B_{j} x_{j} d x_{j+s}
$$

- $\Omega \simeq \mathbb{R}^{m}$ and the metric $g$ is flat : $g=\sum d x_{j} \otimes d x_{j}$.

- $E \simeq \Omega \times \mathbb{C}^{r}$ is a trivial (flat) Hermitian bundle.

- the Hermitian form $V$ is constant. We choose an orthonormal frame of $E$ in which $V$ is diagonal, i.e.

$$
\langle V u, u\rangle=\sum_{1 \leqslant \lambda \leqslant r} V_{\lambda}\left|u_{\lambda}\right|^{2}
$$

In this ideal situation, the connection $D_{k}$ on $E \otimes L^{k}$ can be written $D_{k} u=d u-i k A \wedge u$ and the quadratic form $Q_{k, \Omega}$ is given by

$Q_{k, \Omega}(u)=\int_{\mathbb{R}^{m}} \frac{1}{k}\left(\sum_{\substack{1 \leqslant j \leqslant s \\ 1 \leqslant \lambda \leqslant r}}\left(\left|\frac{\partial u_{\lambda}}{\partial x_{j}}\right|^{2}+\left|\frac{\partial u_{\lambda}}{\partial x_{j+s}}-i k B_{j} x_{j} u_{\lambda}\right|^{2}\right)+\sum_{\substack{j>2 s \\ 1 \leqslant \lambda \leqslant r}}\left|\frac{d u_{\lambda}}{d x_{j}}\right|^{2}\right)-\sum_{1 \leqslant \lambda \leqslant r} V_{\lambda}\left|u_{\lambda}\right|^{2}$.

In this situation, $Q_{k, \Omega}$ is a direct sum of quadratic forms acting on each component $u_{\lambda}$ and the computation of $e^{-t \square_{k}}$ is reduced to the following model cases (1.10), (1.11) in dimension 1 or 2 :

$$
Q(f)=\int_{\mathbb{R}}\left|\frac{d f}{d x}\right|^{2}, \quad \square f=-\frac{d^{2} f}{d x^{2}}
$$

As is well known (and although the spectrum is not discrete in that case) the kernel of the "elementary" heat operator $e^{-t \square}$ is given by

$$
K_{t, \mathbb{R}}(x, y)=\frac{1}{\sqrt{4 \pi t}} e^{-(x-y)^{2} / 4 t}
$$

as follows from solving equation (0.19). The second model case is :

$$
Q(f)=\int_{\mathbb{R}^{2}}\left|\frac{d f}{d x_{1}}\right|^{2}+\left|\frac{d f}{d x_{2}}-i a x_{1} f\right|^{2}
$$

A partial Fourier transform $\widehat{f}\left(x_{1}, \xi_{2}\right)=\frac{1}{\sqrt{2 \pi}} \int_{\mathbb{R}} f\left(x_{1}, x_{2}\right) e^{-i x_{2} \xi_{2}} d x_{2}$ gives

$$
Q(f)=\int_{\mathbb{R}^{2}}\left|\frac{d \widehat{f}}{d x_{1}}\left(x_{1}, \xi_{2}\right)\right|^{2}+a^{2}\left(x_{1}-\frac{\xi_{2}}{a}\right)^{2}\left|\widehat{f}\left(x_{1}, \xi_{2}\right)\right|^{2}
$$

and the change of variables $x_{1}^{\prime}=x_{1}-\xi_{2} / a, x_{2}^{\prime}=\xi_{2}$ leads (after dropping the second variable $x_{2}^{\prime}$ ) to the so called "harmonic oscillator" energy functional

$$
q(g)=\int_{\mathbb{R}}\left|\frac{d g}{d x}\right|^{2}+a^{2} x^{2}|g|^{2}, \quad \square=-\frac{d^{2}}{d x^{2}}+a^{2} x^{2} .
$$


The heat kernel of this operator is given by Mehler's formula:

$$
k_{t, \mathbb{R}}(x, y)=\sqrt{\frac{a}{2 \pi \sinh 2 a t}} \exp \left(-\frac{a}{2}(\operatorname{coth} 2 a t)(x-y)^{2}-a(\tanh a t) x y\right),
$$

which actually reduces to $\left(1.10^{\prime}\right)$ when $a \rightarrow 0$. One way of obtaining this relation is to observe that the unitary eigenfunctions of $\square$ are

$$
\left(2^{p} p ! \sqrt{\frac{\pi}{a}}\right)^{-1 / 2} \Phi_{p}(\sqrt{a} x), \quad p=0,1,2, \ldots,
$$

with associated eigenvalues $(2 p+1) a$, where $\left(\Phi_{p}\right)$ is the sequence of functions associated with Hermite polynomials:

$$
\Phi_{p}(x)=e^{x^{2} / 2} \frac{d^{p}}{d x^{p}}\left(e^{-x^{2}}\right) .
$$

In fact, for $a=1$, easy calculations bearing on derivatives of $e^{x^{2} / 2}$ show that

$$
\left(-\frac{d^{2}}{d x^{2}}+x^{2}\right) \Phi_{p}(x)=-e^{x^{2} / 2} \frac{d^{p+2}}{d x^{p+2}}\left(e^{-x^{2}}\right)-2 x e^{x^{2} / 2} \frac{d^{p+1}}{d x^{p+1}}\left(e^{-x^{2}}\right)-e^{x^{2} / 2} \frac{d^{p}}{d x^{p}}\left(e^{-x^{2}}\right) .
$$

We can now replace the first term by $e^{x^{2} / 2} \frac{d^{p+1}}{d x^{p+1}}\left(2 x \cdot e^{-x^{2}}\right)$ and use the Leibniz formula for the differentiation of the product to see that $\square \Phi_{p}(x)=(2 p+1) \Phi_{p}(x)$. Therefore

$$
k_{t, \mathbb{R}}(x, y)=\sqrt{\frac{a}{\pi}} e^{a\left(x^{2}+y^{2}\right) / 2} \sum_{p=0}^{+\infty} \frac{e^{-(2 p+1) a t}}{2^{p} p ! a^{p}} \frac{d^{p}}{d x^{p}}\left(e^{-a x^{2}}\right) \frac{d^{p}}{d y^{p}}\left(e^{-a y^{2}}\right)
$$

The above summation $\Sigma(x, y)=\sum_{p=0}^{+\infty} \ldots$ can be computed via its Fourier transform

$$
\begin{aligned}
\widehat{\Sigma}(\xi, \eta) & =\frac{1}{2 a} e^{-a t} \sum_{p=0}^{+\infty} \frac{1}{p !}\left(\frac{e^{-2 a t}}{2 a}\right)^{p}(i \xi)^{p}(i \eta)^{p} e^{-\xi^{2} / 4 a} e^{-\eta^{2} / 4 a} \\
& =\frac{1}{2 a} e^{-a t} \exp \left(-\frac{1}{4 a}\left(\xi^{2}+\eta^{2}+2 e^{-2 a t} \xi \eta\right)\right)
\end{aligned}
$$

thus

$$
\Sigma(x, y)=\frac{e^{-a t}}{\sqrt{1-e^{-4 a t}}} \exp \left(-\frac{a}{1-e^{-4 a t}}\left(x^{2}+y^{2}-2 e^{-2 a t} x y\right)\right) .
$$

and Mehler's formula $\left(1.12^{\prime}\right)$ follows. Through our change of variables, the heat operator of $Q$ is given by

$$
\widehat{K_{t, \mathbb{R}^{2}}} f\left(x_{1}, \xi_{2}\right)=\int_{\mathbb{R}} k_{t, \mathbb{R}}\left(x_{1}-\frac{\xi_{2}}{a}, y_{1}-\frac{\xi_{2}}{a}\right) \widehat{f}\left(y_{1}, \xi_{2}\right) d y_{1} .
$$

By an inverse partial Fourier transform left to the reader, we obtain the desired heat kernel expression

$$
\begin{aligned}
K_{t, \mathbb{R}^{2}}\left(x_{1}, x_{2} ; y_{1}, y_{2}\right)=\frac{a}{4 \pi \sinh a t} \exp \left(-\frac{a}{4}(\operatorname{coth} a t)\right. & \left.\left(\left(x_{1}-y_{1}\right)^{2}+\left(x_{2}-y_{2}\right)^{2}\right)\right) \\
& \times \exp \left(\frac{i}{2} a\left(x_{1}+y_{1}\right)\left(x_{2}-y_{2}\right)\right) .
\end{aligned}
$$


The heat kernel associated with a sum of (pairwise commuting) operators $\square_{1}, \ldots, \square_{m}$ acting on disjoint sets of variables is the product of the corresponding heat kernels $e^{-t \square_{j}}$. Let $K_{t, k, \Omega}^{\lambda}$ be the heat kernel of the component of $Q_{k, \Omega}$ acting on each single entry $u_{\lambda}$. The factor in the heat kernel corresponding to each pair of variables $\left(x_{j}, x_{j+s}\right), 1 \leqslant j \leqslant s$, is obtained by substituting $k B_{j}$ to $a$ and $t / k$ to $t$ (the latter rescaling comes from the initial factor $\frac{1}{k}$ in the expression of $\left.Q_{k, \Omega}\right)$. For the other coordinates $j>2 s$ where $B$ has no coefficients, the kernel falls back to the "elementary" heat kernel $\left(1.10^{\prime}\right)$. Finally, the constant term $-V_{\lambda}\left|u_{\lambda}\right|^{2}$ contributes to multiplying the heat kernel by $e^{t V_{\lambda}}$. Therefore we get for the global heat kernel on $\Omega=\mathbb{R}^{n}$ the explicit formula

$$
\begin{aligned}
& K_{t, k, \mathbb{R}^{n}}^{\lambda}(x, y)=\prod_{j=1}^{s} \frac{k B_{j}}{4 \pi \sinh B_{j} t} \exp \left(-\frac{k B_{j}}{4}\left(\operatorname{coth} B_{j} t\right)\left(\left(x_{2 j-1}-y_{2 j-1}\right)^{2}+\left(x_{2 j}-y_{2 j}\right)^{2}\right)\right. \\
&\left.+\frac{i}{2} k B_{j}\left(x_{2 j-1}+y_{2 j-1}\right)\left(x_{2 j}-y_{2 j}\right)\right) \\
& \times e^{t V_{\lambda}} \times \frac{1}{(4 \pi t / k)^{m-2 s} / 2} \exp \left(-k \sum_{j>2 s}\left(x_{j}-y_{j}\right)^{2} / 4 t\right) .
\end{aligned}
$$

On the diagonal of $\mathbb{R}^{n} \times \mathbb{R}^{n}$, the global heat kernel $K_{t, k, \mathbb{R}^{n}}$ is thus given by the rather simple $\left(\operatorname{Herm}(E) \otimes \operatorname{Id}_{L^{k}}\right)$-valued tensor depending only on $B, V$ and $t / k$ :

$$
K_{t, k, \mathbb{R}^{n}}(x, x)=\left(\frac{k}{4 \pi t}\right)^{m / 2} e^{t V} \prod_{j=1}^{s} \frac{B_{j} t}{\sinh B_{j} t} .
$$

(1.15) Theorem. Consider the general (variable coefficient) case. For $\delta>0$ small, the heat kernel of $\square_{k}$ over $M$ admits an asymptotic estimate

$$
K_{t, k, M}(x, x)=\left(\frac{k}{4 \pi t}\right)^{m / 2} e^{t V(x)} \prod_{j=1}^{s} \frac{B_{j}(x) t}{\sinh B_{j}(x) t}\left(1+O\left(k^{-1 / 2+\delta}\right)\right)
$$

as $k \rightarrow+\infty$, where $O\left(k^{-1 / 2+\delta}\right)$ is uniform with respect to $x \in M$ and $t$ in a bounded interval $] 0, T] \subset] 0,+\infty[$ (moreover, for every open set $\Omega \subset M$, a similar estimate is valid for $K_{t, k, \Omega}$ on relatively compact subsets of $\Omega$ ).

Proof. Notice first that $(t, x) \mapsto \prod_{j=1}^{s} \frac{B_{j}(x) t}{\sinh B_{j}(x) t}$ extends as a smooth positive function on $[0,+\infty[\times M$, equal to 1 when $t=0$ : this is in fact the inverse of the square root of the determinant of the positive definite symmetric matrix

$$
\frac{\sin (t b(x))}{t b(x)}=\sum_{p=0}^{+\infty} \frac{t^{2 p}\left(-b(x)^{2}\right)^{p}}{(2 p+1) !} \geqslant \mathrm{Id},
$$

where $b(x)$ is the antisymmetric endomorphism of $T_{M}$ associated with the alternate 2 -form $B(x)$ and $-b(x)^{2}=b(x)^{\dagger} b(x) \geqslant 0$.

The only thing one has still to get convinced of is that the kernel of $e^{-t \square_{k}}-e^{-t \square_{k}^{0}}$ is $(k / t)^{m / 2} O\left(k^{-1 / 2+\delta}\right)$ uniformly along the diagonal at any point $\left(x^{0}, x^{0}\right) \in M \times M$, where $\square_{k}^{0}$ is the operator $\square_{k}$ "freezed" at $x^{0}$. We can do this in a canonical way by using normal coordinates from the Riemannian exponential mapping

$$
\exp _{x^{0}}: \mathbb{R}^{m} \simeq T_{M, x^{0}} \rightarrow M,
$$


and trivializations of $E$ and $L$ produced by parallel transport along geodesics from $x^{0}$ to any point $x \in B\left(x^{0}, \rho_{0}\right)$, where $\rho_{0}=$ injectivity radius of $M$. In this way, we actually get automatically that $\Gamma_{L}\left(x^{0}\right)=\Gamma_{E}\left(x^{0}\right)=0$. When Supp $u \subset \Omega_{\rho}:=B\left(x^{0}, \rho\right)$, a Taylor expansion yields $D_{k} u-D_{k}^{0} u=O\left(|x|+k|x|^{2}\right) \cdot u$ and we get the estimates

$$
\begin{aligned}
Q_{k, \Omega_{\rho}}(u)-Q_{k, \Omega_{\rho}}^{0}(u) & =\int_{M} \frac{1}{k}\left(\left|D_{k} u\right|^{2}-\left|D_{k}^{0} u\right|^{2}\right)-\left\langle\left(V-V^{0}\right) u, u\right\rangle \\
& =O\left(\int_{M} \frac{1}{k}\left(\left(\rho+k \rho^{2}\right)\left|D_{k}^{0} u\right||u|+\left(\rho+k \rho^{2}\right)^{2}|u|^{2}\right)+\rho|u|^{2}\right) \\
& =O\left(\int_{M} \frac{\varepsilon}{k}\left|D_{k}^{0} u\right|^{2}+\left(\frac{\left(\rho+k \rho^{2}\right)^{2}}{k \varepsilon}+\rho\right)|u|^{2}\right) \\
& =O\left(\varepsilon Q_{k, \Omega_{\rho}}^{0}(u)+\left(\frac{\left(\rho+k \rho^{2}\right)^{2}}{k \varepsilon}+\rho+\varepsilon\right)|u|^{2}\right)
\end{aligned}
$$

whenever $\varepsilon<1$, hence there is a constant $C_{\rho, k, \varepsilon}=O\left(\frac{\left(\rho+k \rho^{2}\right)^{2}}{k \varepsilon}+\rho+\varepsilon\right)$ such that

$$
(1-\varepsilon) Q_{k, \Omega_{\rho}}^{0}(u)-C_{\rho, k, \varepsilon}|u|^{2} \leqslant Q_{k, \Omega_{\rho}}(u) \leqslant(1+\varepsilon) Q_{k, \Omega_{\rho}}^{0}(u)+C_{\rho, k, \varepsilon}|u|^{2} .
$$

From this, we conclude that $e^{-t \square_{k}}$ is squeezed (as a positive bounded self-adjoint operator) between $e^{-C_{\rho, k, \varepsilon} t} e^{-t(1+\varepsilon) \square_{k}^{0}}$ and $e^{C_{\rho, k, \varepsilon} t} e^{-t(1-\varepsilon) \square_{k}^{0}}$. By definition of the heat kernel we have

$$
\begin{aligned}
K_{t, k, \Omega_{\rho}}\left(x^{0}, x^{0}\right) & =\lim _{\nu \rightarrow+\infty} \int_{\Omega_{\rho} \times \Omega_{\rho}} K_{t, k, \Omega_{\rho}}(x, y) u_{\nu}(x) \overline{u_{\nu}(y)} d \sigma(x) d \sigma(y) \\
& =\lim _{\nu \rightarrow+\infty}\left\langle\left\langle e^{-t \square_{k}} u_{\nu}, u_{\nu}\right\rangle\right\rangle
\end{aligned}
$$

when $u_{\nu} \underset{L^{1}}{\longrightarrow} \delta_{x^{0}}$ (Dirac measure), thus

$$
\begin{gathered}
e^{-C_{\rho, k, \varepsilon} T} K_{(1+\varepsilon) t, k, \Omega_{\rho}}^{0}\left(x^{0}, x^{0}\right)-K_{t, k, \Omega_{\rho}}^{0}\left(x^{0}, x^{0}\right) \leqslant K_{t, k, \Omega_{\rho}}\left(x^{0}, x^{0}\right)-K_{t, k, \Omega_{\rho}}^{0}\left(x^{0}, x^{0}\right) \\
\leqslant e^{C_{\rho, k, \varepsilon} T} K_{(1-\varepsilon) t, k, \Omega_{\rho}}^{0}\left(x^{0}, x^{0}\right)-K_{t, k, \Omega_{\rho}}^{0}\left(x^{0}, x^{0}\right) .
\end{gathered}
$$

We take here $\rho=\varepsilon=k^{-1 / 2+\delta}$, so that $C_{\rho, k, \varepsilon}=O\left(k^{-1 / 2+\delta}\right)$. The expected uniform bounds are then obtained by an application of Proposition 1.9, where the choice $\rho=k^{-1 / 2+\delta} \gg k^{-1 / 2}$ ensures that the relative errors

$$
K_{t, k, M}-K_{t, k, \Omega_{\rho}} \quad \text { and } \quad K_{t, k, \mathbb{R}^{m}}^{0}-K_{t, k, \Omega_{\rho}}^{0}
$$

are very small, namely of the order of magnitude $O\left(\exp \left(-k^{\delta} / 4 T\right)\right)$.

As a consequence, we obtain the following estimate for the eigenvalues :

(1.16) Corollary. The eigenvalues $\lambda_{\nu, k, \Omega}$ of $Q_{k, \Omega}$ satisfy for every $t>0$ the estimate

$$
\sum_{\nu=1}^{+\infty} e^{-t \lambda_{\nu, k, \Omega}}=\left(1+O\left(k^{-1 / 2}\right)\right)\left(\frac{k}{4 \pi t}\right)^{m / 2} \int_{\Omega} \operatorname{tr}\left(e^{t V(x)}\right) \prod_{j=1}^{s} \frac{B_{j}(x) t}{\sinh B_{j}(x) t} d \sigma(x) .
$$


This result can be also interpreted in terms of the counting function

$$
N_{k, \Omega}(\lambda)=\#\left\{\nu ; \lambda_{\nu, k, \Omega} \leqslant \lambda\right\}
$$

and of the spectral density measure (a sum of Dirac measures on the real line)

$$
\mu_{k, \Omega}=k^{-m / 2} \frac{d}{d \lambda} N_{k, \Omega}(\lambda)
$$

Notice that the measures $\mu_{k, \Omega}$ are all supported in the fixed interval $\left[-v_{0},+\infty\left[\right.\right.$, where $v_{0}$ is an upper bound for the eigenvalues of $V(x), x \in M$. In these notations, Corollary 1.16 can be restated :

$$
\lim _{k \rightarrow+\infty} \int_{-\infty}^{+\infty} e^{-t \lambda} d \mu_{k, \Omega}(\lambda)=\frac{1}{(4 \pi t)^{m / 2}} \int_{\Omega} \operatorname{tr}\left(e^{t V(x)}\right) \prod_{j=1}^{s} \frac{B_{j}(x) t}{\sinh B_{j}(x) t} d \sigma(x) .
$$

We thus see that the sequence of measures $\mu_{k, \Omega}$ converges weakly to a measure $\mu_{\Omega}$ whose Laplace transform is given by the right hand side. Inverting the formula, one obtains :

(1.17) Corollary. For almost all $\lambda \in \mathbb{R}$

$$
\left.\left.\lim _{k \rightarrow+\infty} k^{-m / 2} N_{k, \Omega}(\lambda)=\mu_{\Omega}(]-\infty, \lambda\right]\right)=\int_{\Omega} \sum_{j=1}^{r} \nu_{B(x)}\left(V_{j}(x)+\lambda\right) d \sigma(x)
$$

where $\nu_{B(x)}(\lambda)$ is the function on $M \times \mathbb{R}$ defined by

$$
\nu_{B}(\lambda)=\frac{2^{s-m} \pi^{-m / 2}}{\Gamma\left(\frac{m}{2}-s+1\right)} B_{1} \cdots B_{s} \sum_{\left(p_{1}, \ldots, p_{s}\right) \in \mathbb{N}^{s}}\left[\lambda-\sum\left(2 p_{j}+1\right) B_{j}\right]_{+}^{\frac{m}{2}-s} .
$$

Proof. We leave as an exercise to the reader to check that the Laplace transform

$$
\int_{-\infty}^{+\infty} e^{-t \lambda} d \nu_{B}(v+\lambda)=e^{t v} \int_{-\infty}^{+\infty} e^{-t \lambda} d \nu_{B}(\lambda)
$$

is actually equal to

$$
\frac{e^{t v}}{(4 \pi)^{m / 2}} \prod_{j=1}^{s} \frac{B_{j}(x) t}{\sinh B_{j}(x) t}
$$

\section{C. Proof of the holomorphic Morse inequalities}

Let $X$ be a compact complex manifold, $L$ and $E$ holomorphic Hermitian vector bundles of rank 1 and $r$ over $X$. If $X$ is endowed with a Hermitian metric $\omega$, Hodge theory shows that the Dolbeault cohomology group $H^{q}\left(X, E \otimes L^{k}\right)$ can be identified with the space of harmonic $(0, q)$-forms with respect to the Laplace-Beltrami operator $\Delta_{k}^{\prime \prime}=\bar{\partial}_{k} \bar{\partial}_{k}^{*}+\bar{\partial}_{k}^{*} \bar{\partial}_{k}$ acting on $E \otimes L^{k}$. We thus have to estimate the zero-eigenspace of $\Delta_{k}^{\prime \prime}$. 
In order to apply corollary 3.2 , we first have to compute $\Delta_{k}^{\prime \prime}$ in terms of the Hermitian connection $\nabla_{k}$ on $E \otimes L^{k} \otimes \Lambda^{0, q} T_{X}^{*}$ deduced from the Chern connections of $L, E, T_{X}$. What plays now the role of $E$ is the (non holomorphic) bundle $E \otimes \Lambda^{0, q} T_{X}^{*}$.

The relation between $\Delta_{k}^{\prime \prime}$ and $\nabla_{k}$ is most easily obtained by means of the BochnerKodaira-Nakano identity. In order to simplify the exposition, we assume here that the metric $\omega$ on $X$ is Kähler. For any Hermitian holomorphic line bundle $G$ on $X$, the operators $\Delta^{\prime}$ and $\Delta^{\prime \prime}$ associated with the Chern connection $D=D_{G}$ are related by the B-K-N identity (cf. [Boc48], [Kod53], [AN54], [Nak55])

$$
\Delta^{\prime \prime}=\Delta^{\prime}+\left[i \theta_{G}, \Lambda\right]
$$

where $\theta_{G}=D_{G}^{2} \in C^{\infty}\left(X, \Lambda^{1,1} T_{X}^{*} \otimes \operatorname{Hom}(G, G)\right)$ is the curvature tensor and $\Lambda=L^{*}$ is the adjoint of the Lefschetz operator $L u=\omega \wedge u$.

The Leibniz rule implies $\theta_{E \otimes L^{k}}=k \theta_{L} \otimes \operatorname{Id}_{E}+\theta_{E} \otimes \operatorname{Id}_{L^{k}}$ (omitting the Hermitian metrics for simplicity of notation), thus

$$
\Delta_{k}^{\prime \prime}=\Delta_{k}^{\prime}+k\left[i \theta_{L}, \Lambda\right]+\left[i \theta_{E}, \Lambda\right]
$$

At a given point $z^{0} \in X$, we can find a coordinate system $\left(z_{1}, \ldots, z_{n}\right)$ such that $\left(\partial / \partial z_{j}\right)$ is an orthonormal basis of $T_{X}$ diagonalizing $i \theta_{L}\left(z^{0}\right)$, in such a way that

$$
\omega\left(z^{0}\right)=\frac{i}{2} \sum_{1 \leqslant j \leqslant n} d z_{j} \wedge d \bar{z}_{j}, \quad i \theta_{L}\left(z^{0}\right)=\frac{i}{2} \sum_{1 \leqslant j \leqslant n} \alpha_{j} d z_{j} \wedge d \bar{z}_{j}
$$

where $\alpha_{1}, \ldots, \alpha_{n}$ are the curvature eigenvalues of $i \theta_{L}\left(z^{0}\right)$. A standard formula gives the expression of the curvature term $\left[i \theta_{L}, \Lambda\right] u$ for any $(p, q)$-form $u$. In fact, for $u=$ $\sum u_{I, J, \lambda} d z_{I} \wedge d \bar{z}_{J} \otimes e_{\lambda}$, we have

$$
\left\langle\left[i \theta_{L}, \Lambda\right] u, u\right\rangle=\sum_{I, J, \lambda}\left(\alpha_{J}-\alpha_{\complement I}\right)\left|u_{I, J, \lambda}\right|^{2}
$$

where $\alpha_{J}=\sum_{j \in J} \alpha_{j}$. In the case of a $(0, q)$-form $u=\sum u_{J, \lambda} d \bar{z}_{J} \otimes e_{\lambda}$ we simply have $\Delta_{k}^{\prime} u=D_{k}^{\prime *} D_{k}^{\prime} u=\nabla_{k}^{\prime *} \nabla_{k}^{\prime} u$ and

$$
\begin{aligned}
\Delta_{k}^{\prime \prime} & =\nabla_{k}^{\prime *} \nabla_{k}^{\prime}-k V^{\prime}+\left[i \theta_{E}, \Lambda\right], \\
\left\langle V^{\prime} u, u\right\rangle & =\sum_{J, \lambda} \alpha_{\complement J}\left|u_{J, \lambda}\right|^{2} \quad(\text { here } I=\emptyset) .
\end{aligned}
$$

This is not yet what was needed, since only the $(1,0)$ part $\nabla_{k}^{\prime}$ appears. To get the $(0,1)$ component, we consider $u$ as a $(n, q)$ form with values in $E \otimes L^{k} \otimes \Lambda^{n} T_{X}$. We then get $\Delta_{k}^{\prime} u=D_{k}^{\prime} D_{k}^{\prime *} u$ where

$$
D_{k}^{\prime *} u=-\sum \partial u_{I, J, \lambda} / \partial \bar{z}_{j} d z_{1} \wedge \cdots \widehat{d z_{j}} \cdots \wedge d z_{n} \wedge d \bar{z}_{J} \otimes e_{\lambda}
$$

in normal coordinates. Thus $\Delta_{k}^{\prime} u=\nabla_{k}^{\prime *} \nabla_{k}^{\prime \prime} u$ and

$$
\Delta_{k}^{\prime \prime}=\nabla_{k}^{\prime \prime *} \nabla_{k}^{\prime \prime}+k V^{\prime \prime}+\left[i \theta_{E \otimes \Lambda^{n} T_{X}}, \Lambda\right]
$$




$$
\left\langle V^{\prime \prime} u, u\right\rangle=\sum_{J, \lambda} \alpha_{J}\left|u_{J, \lambda}\right|^{2} \quad(\text { here } I=\{1, \ldots, n\}) .
$$

If the metric $\omega$ is non Kähler, we get additional torsion terms, but these terms are independent of $k$. A combination of $\left(1.21^{\prime}\right)$ and $\left(1.21^{\prime \prime}\right)$ yields

$$
\frac{2}{k} \Delta_{k}^{\prime \prime}=\frac{1}{k} \nabla_{k}^{*} \nabla_{k}-V+\frac{1}{k} W
$$

where $W$ is a Hermitian form independent of $k$ and

$$
\langle V u, u\rangle=\sum_{J, \lambda}\left(\alpha_{\complement J}-\alpha_{J}\right)\left|u_{J, \lambda}\right|^{2}
$$

Now apply Theorem 1.15 and observe that $W$ does not give any significant contribution to the heat kernel as $k \rightarrow+\infty$. We write here $z_{j}=x_{j}+i y_{j}$ and the "magnetic field"

$$
B=i \theta_{L}=\sum_{1 \leqslant j \leqslant n} \alpha_{j} d x_{j} \wedge d y_{j}
$$

The curvature eigenvalues are given by $B_{j}=\left|\alpha_{j}\right|$. We denote $s=s(x)$ the rank of $B(x)$ and order the eigenvalues so that

$$
\left|\alpha_{1}\right| \geqslant \cdots \geqslant\left|\alpha_{s}\right|>0=\alpha_{s+1}=\cdots=\alpha_{n} .
$$

The eigenvalues of $V$ acting on $E \otimes \Lambda^{n} T_{X}^{*}$ are the coefficients $\alpha_{\complement J}-\alpha_{J}$, counted with multiplicity $r$. Therefore

(1.23) Theorem. The heat kernel associated with $e^{-\frac{2 t}{k} \Delta_{k}^{\prime \prime}}$ in bidegree $(0, q)$ satisfies

$$
K_{t}^{k}(x, x) \sim k^{n} \frac{r \sum_{|J|=q} e^{t\left(\alpha_{C_{J}}(x)-\alpha_{J}(x)\right)}}{(4 \pi)^{n} t^{n-s}} \prod_{j=1}^{s} \frac{\left|\alpha_{j}(x)\right|}{\sinh \left|\alpha_{j}(x)\right| t}
$$

as $k \rightarrow+\infty$. In particular, if $\lambda_{1}^{k, q} \leqslant \lambda_{2}^{k, q} \leqslant \cdots$ are the eigenvalues of $\frac{1}{k} \Delta_{k}^{\prime \prime}$ in bidegree $(0, q)$, we have

$$
\sum_{\nu=1}^{+\infty} e^{-2 t \lambda_{\nu}^{k, q}} \sim r k^{n} \sum_{|J|=q} \int_{X} \frac{e^{t\left(\alpha_{\mathrm{C}_{J}}(x)-\alpha_{J}(x)\right)}}{(4 \pi)^{n} t^{n-s}} \prod_{j=1}^{s} \frac{\left|\alpha_{j}(x)\right|}{\sinh \left|\alpha_{j}(x)\right| t}
$$

for every $t>0$.

At this point, the main idea is to use the eigenspaces to construct a finite dimensional subcomplex of the Dolbeault complex possessing the same cohomology groups. This was already the basic idea in Witten's analytic proof of the standard Morse inequalities [Wit82]. We denote by

$$
\mathscr{H}_{\lambda}^{k, q}, \quad \text { resp. } \mathscr{H}_{\leqslant \lambda}^{k, q}
$$


the $\lambda$-eigenspace of $\frac{1}{k} \Delta_{k}^{\prime \prime}$ acting on $C^{\infty}\left(X, \Lambda^{0, q} T_{X}^{*} \otimes E \otimes L^{k}\right)$, resp. the direct sum of eigenspaces corresponding to all eigenvalues $\leqslant \lambda$. As $\bar{\partial}_{k}$ and $\Delta_{k}^{\prime \prime}$ commute, we see that $\bar{\partial}\left(\mathscr{H}_{\lambda}^{k, q}\right) \subset \mathscr{H}_{\lambda}^{k, q+1}$, thus $\mathscr{H}_{\lambda}^{k, \bullet}$ and $\mathscr{H}_{\leqslant \lambda}^{k, \bullet}$ are finite dimensional subcomplexes of the Dolbeault complex

$$
\bar{\partial}: C^{\infty}\left(X, \Lambda^{0, \bullet} T_{X}^{*} E \otimes L^{k}\right) .
$$

Since $\bar{\partial}_{k} \bar{\partial}_{k}^{*}+\bar{\partial}_{k}^{*} \bar{\partial}_{k}=\Delta_{k}^{\prime \prime}=k \lambda$ Id on $\mathscr{H}_{\lambda}^{k, \bullet}$, we see that $\mathscr{H}_{\lambda}^{k, \bullet}$ has trivial cohomology for $\lambda \neq 0$. Since $\mathscr{H}_{0}^{k, \bullet}$ is the space of harmonic forms, we see that $\mathscr{H}_{\leqslant \lambda}^{k, \bullet}$ has the same cohomology as the Dolbeault complex for $\lambda>0$. We will call this complex the Witten $\bar{\partial}$-complex. We need an elementary lemma of linear algebra.

(1.24) Lemma. Set $h_{k}^{q}=\operatorname{dim} H^{q}\left(X, E \otimes L^{k}\right)$. Then for every $t>0$

$$
h_{k}^{q}-h_{k}^{q-1}+\cdots+(-1)^{q} h_{k}^{0} \leqslant \sum_{\ell=0}^{q}(-1)^{q-\ell} \sum_{j=1}^{+\infty} e^{-t \lambda_{j}^{k, \ell}} .
$$

Proof. The left hand side is the contribution of the 0 eigenvalues in the right hand side. All we have to check is that the contribution of the other eigenvalues is $\geqslant 0$. The contribution of the eigenvalues such that $\lambda_{j}^{k, \ell}=\lambda>0$ is

$$
e^{-t \lambda} \sum_{\ell=0}^{q}(-1)^{q-\ell} \operatorname{dim} \mathscr{H}_{\lambda}^{k, \ell}
$$

As $\mathscr{H}_{\lambda}^{k, \bullet}$ is exact, one easily sees that the last sum is equal to the dimension of $\bar{\partial} \mathscr{H}_{\lambda}^{k, q} \subset$ $\mathscr{H}_{\lambda}^{k, q+1}$, hence $\geqslant 0$.

Combining Theorem 1.23 with Lemma 1.24, we get $h_{k}^{q}-h_{k}^{q-1}+\cdots+(-1)^{q} h_{k}^{0} \leqslant o\left(k^{n}\right)+$

$$
r k^{n} \sum_{\ell=0}^{q}(-1)^{q-\ell} \sum_{|J|=\ell} \int_{X} \frac{\prod_{j \leqslant s}\left|\alpha_{j}\right| \cdot e^{t\left(\alpha_{\mathrm{C}_{J}}-\alpha_{J}-\sum\left|\alpha_{j}\right|\right)}}{2^{2 n-s} \pi^{n} t^{n-s} \prod_{j \leqslant s}\left(1-e^{-2 t\left|\alpha_{j}\right|}\right)} .
$$

This inequality is valid for any $t>0$, so we can let $t$ tend to $+\infty$. It is clear that $\alpha_{C J}-\alpha_{J}-\sum\left|\alpha_{j}\right|$ is always $\leqslant 0$, thus the integrand tends to 0 at every point where $s<n$. When $s=n$, we have $\left.\alpha_{\complement}(x)-\alpha_{J} x\right)-\sum\left|\alpha_{j}(x)\right|=0$ if and only if $\alpha_{j}(x)>0$ for every $j \in \complement J$ and $\alpha_{j}(x)<0$ for every $j \in J$. This implies $x \in X(L, h, \ell)$; in this case there is only one multi-index $J$ satisfying the above conditions and the limit is

$$
(2 \pi)^{-n}\left|\alpha_{1} \cdots \alpha_{n}\right|=(2 \pi)^{-n}\left|\left(i \theta_{L, h}\right)^{n}\right|=\left|\Theta_{L, h}^{n}\right|,
$$

as $\Theta_{L, h}=\frac{i}{2 \pi} \theta_{L, h}$ by definition. By the monotone convergence theorem, our sum of integrals converges to

$$
\sum_{\ell=0}^{q}(-1)^{q-\ell} \int_{X(L, h, \ell)}(2 \pi)^{-n}\left|\alpha_{1} \cdots \alpha_{n}\right| d \sigma=\frac{1}{n !} \int_{X(L, h, \leqslant q)}(-1)^{q} \Theta_{L, h}^{n} .
$$

The Main Theorem 1.2 follows. 


\section{Applications to algebraic geometry}

\section{A. Solution of the Grauert-Riemenschneider conjecture}

Let $L$ be a holomorphic line bundle over a compact connected complex manifold $X$ of dimension $n$ and $V_{k}=H^{0}\left(X, L^{k}\right)$. Denote by $Z\left(V_{k}\right)$ the set of common zeroes of all sections in $V_{k}$, and fix a basis $\left(\sigma_{0}, \ldots, \sigma_{N}\right)$ of $V_{k}$. There is a canonical holomorphic map

$$
\Phi_{k L}: X \backslash Z\left(V_{k}\right) \longrightarrow \mathbb{P}\left(V_{k}\right), \quad x \mapsto\left[\sigma_{0}(x): \ldots: \sigma_{N}(x)\right]
$$

sending a point $x \in X \backslash Z\left(V_{k}\right)$ to the hyperplane $H \subset V_{k}$ of sections $\sigma=\sum \lambda_{j} \sigma_{j} \in V_{k}$ such that $\sigma(x)=\sum \lambda_{j} \sigma_{j}(x)=0$; it is therefore given by $x \mapsto\left[\sigma_{0}(x): \ldots: \sigma_{N}(x)\right]$ in projective coordinates on $\mathbb{P}\left(V_{k}\right) \simeq \mathbb{P}^{N}$. The pull-back $\Phi_{k L}^{*} \mathscr{O}(d)$ can be identified with the restriction of $L^{k d}$ to $X \backslash Z\left(V_{k}\right)$; indeed, to any homogeneous polynomial $P\left(w_{0}, \ldots, w_{N}\right) \in$ $H^{0}\left(\mathbb{P}^{N}, \mathscr{\sigma}(d)\right)$ of degree $d$, one can associate a section

$$
s=P\left(\sigma_{0}, \ldots, \sigma_{N}\right) \in H^{0}\left(X, L^{k d}\right) .
$$

When $L$ possesses a smooth Hermitian metric $h$ with $\Theta_{L, h}>0$, one can construct many sections of high tensor powers $L^{k}$ (e.g. by Hörmander's $L^{2}$ estimates [Hör65], [AV65] for $\bar{\partial})$. For $k \geqslant k_{0}$ large enough, the "base locus" $Z\left(V_{k}\right)$ is empty, the sections in $V_{k}$ separate any two points of $X$ and generate all 1-jets at any point. Then $\Phi_{k L}$ gives an embedding of $X$ in some projective space $\mathbb{P}^{N}$, for $N=N(k)$ and $k \geqslant k_{0}$. In this way, the theory of $L^{2}$ estimates implies the Kodaira embedding theorem: a compact complex manifold $X$ is projective algebraic if and only if $X$ possesses a Hermitian line bundle $(L, h)$ with $C^{\infty}$ positive curvature.

The Grauert-Riemenschneider conjecture [GR70] is an attempt to characterize the more general class of Moishezon varieties in terms of semi-positive line bundles. Let us first recall a few definitions. The algebraic dimension $a(X)$ is the transcendence degree of the field $\mathscr{M}(X)$ of meromorphic functions on $X$. A well-known theorem of Siegel [Sie55] asserts that $0 \leqslant a(X) \leqslant n$ (see Corollary 2.6 below). A compact manifold or variety $X$ is said to be Moishezon if $a(X)=n$.

By definition, the Kodaira dimension $\kappa(L)$ is the supremum of the dimension of the images $Y_{k}=\Phi_{k L}\left(X \backslash Z\left(V_{k}\right)\right) \subset \mathbb{P}\left(V_{k}^{*}\right)$ for all integers $k>0$ [one defines $\kappa(L)=$ $-\infty$ when $V_{k}=0$ for all $k$, in which case we always have $\left.Y_{k}=\emptyset\right]$. Since the field of meromorphic functions on $X$ obtained by restriction of rational functions of $\mathbb{P}\left(V_{k}^{*}\right)$ to $Y_{k}$ has transcendence degree at least equal to $\operatorname{dim} Y_{k}$, we infer that

$$
-\infty \leqslant \kappa(L)=\sup \operatorname{dim} Y_{k} \leqslant a(X) \leqslant n .
$$

(2.4) Definition. The line bundle $L \rightarrow X$ is said to be big if $\kappa(L)$ is maximal, i.e. $\kappa(L)=n=\operatorname{dim} X$.

The following standard lemma is needed (cf. [Ser54], [Sie55]).

(2.5) Lemma (Serre-Siegel). For every line bundle $L \rightarrow X$, there exist constants $C \geqslant c>0$ and $k_{0} \in \mathbb{N}^{*}$ such that

$$
\begin{array}{ll}
\operatorname{dim} H^{0}\left(X, L^{k}\right) \leqslant C k^{\kappa(L)} & \text { for all } k \geqslant 1, \\
\operatorname{dim} H^{0}\left(X, L^{k}\right) \geqslant c k^{\kappa(L)} & \text { for all } k \geqslant 1 \text { multiple of } k_{0} .
\end{array}
$$


Proof. The lower bound is obtained by taking $k_{0}$ such that $p:=\operatorname{dim} Y_{k_{0}}=\kappa(L)$. Then, by the rank theorem, there exists a point $x_{0} \in X \backslash Z\left(V_{k_{0}}\right)$ and a basis $\left(\sigma_{0}, \ldots, \sigma_{N}\right)$ of $H^{0}\left(X, L^{k_{0}}\right)$ such that $\sigma_{0}\left(x_{0}\right) \neq 0$ and $\left(d\left(\sigma_{1} / \sigma_{0}\right) \wedge \ldots \wedge d\left(\sigma_{p} / \sigma_{0}\right)\right)\left(x_{0}\right) \neq 0$. Then by taking $s=P\left(\sigma_{0}, \ldots, \sigma_{p}, 0, \ldots, 0\right)$ in $(2.2)$, we obtain an injection of the space of homogeneous polynomials of degree $d$ in $p+1$ variables into $H^{0}\left(X, L^{k_{0} d}\right)$, whence

$$
h^{0}\left(X, L^{k_{0} d}\right) \geqslant\left(\begin{array}{c}
d+p \\
p
\end{array}\right) \geqslant d^{p} / p !
$$

The proof of the upper bound proceeds as follows : select a Hermitian metric $h$, on $L$ and a finite family of coordinate balls $B_{j}=B\left(z_{j}, r_{j}\right)$ such that $B_{j}^{\prime}=B\left(z_{j}, r_{j} / 2\right)$ cover $X$, and $L_{\mid B_{j}}$ is trivial for each $j$. By moving a little bit the points $z_{j}$, we may assume that $\Phi_{k L}$ has maximal rank at all points $z_{j}$ for all $k$ (the bad set is at most a countable union of analytic sets, so it is nowhere dense). If $L^{k}$ has many sections, one can solve a linear system in many unknowns to get a section $s$ vanishing at a high order $m$ at all centers $z_{j}$. Then the Schwarz lemma gives

$$
\|s\|_{h, \infty}=\sup _{j}\|s\|_{h, B_{j}^{\prime}} \leqslant 2^{-m} C(h)^{k} \sup _{j}\|s\|_{h, B_{j}} \leqslant 2^{-m} C(h)^{k}\|s\|_{h, \infty}
$$

where $C(h)$ is a bound for the oscillation of the metric $h$ on $B_{j}$, which we may assume to be finite after possibly shrinking $B_{j}$. Thus $m \leqslant k \log C(h) / \log 2$ if $s \neq 0$. Since the sections of $L^{k}$ are constant along the fibers of $\Phi_{k L}$, only $m^{\operatorname{dim} Y_{k}} \#\left\{z_{j}\right\}$ equations transversally to the fibers are needed to make $s$ vanish at order $m$. Therefore we can choose $m \approx\left(h^{0}\left(X, L^{k}\right) / \#\left\{z_{j}\right\}\right)^{1 / \operatorname{dim} Y_{k}}$ and still get a non zero section, so that

$$
h^{0}\left(X, L^{k}\right) \approx \#\left\{z_{j}\right\} \cdot m^{\operatorname{dim} Y_{k}} \leqslant C k^{\kappa(L)} .
$$

(2.6) Corollary (Siegel). For every compact complex manifold $X$

$$
a(X):=\operatorname{tr} \operatorname{deg}_{\mathbb{C}} \mathscr{M}(X) \leqslant n
$$

Proof. Fix $s$ algebraically independent elements $f_{1}, \ldots, f_{s} \in \mathscr{M}(X)$ and let $D$ be the sup of the pole divisors of the $f_{j}$ 's. To every polynomial $P\left(f_{1}, \ldots, f_{s}\right)$ of degree $\leqslant k$ corresponds injectively a section $\sigma_{P}=P\left(f_{1}, \ldots, f_{s}\right) \in H^{0}(X, \mathscr{O}(k D))$. A dimension count implies

$$
\frac{k^{s}}{s !} \leqslant\left(\begin{array}{c}
k+s \\
s
\end{array}\right) \leqslant C k^{\kappa(\Theta(D))} \leqslant C k^{n}
$$

by Lemma 2.5. Therefore $s \leqslant n$.

Now, the Grauert-Riemenschneider conjecture [GR70] can be stated as follows.

(2.7) Grauert-Riemenschneider conjecture. A compact complex variety $Y$ is Moishezon if and only if there is a proper non singular modification $X \rightarrow Y$ and a Hermitian line bundle $(L, h)$ over $X$ such that the curvature form $\Theta_{L, h}$ is $>0$ on a dense open subset of $X$. 
Proof. When $Y$ is Moishezon, it is well known that there exists a projective algebraic modification $X$; therefore we can even take $L$ to be ample and then there exists $h$ such that $\Theta_{L, h}>0$ everywhere on $X$.

The converse statement was proved by Siu in [Siu84, Siu85], assuming only $\Theta_{L, h} \geqslant 0$ everywhere and $\Theta_{L, h}>0$ in at least one point. Morse inequalities provide in fact a much stronger criterion, requiring only the positivity of some curvature integral:

(2.8) Theorem. If a Hermitian line bundle $(L, h)$ on $X$ satisfies the integral condition

$$
\int_{X(L, h, \leqslant 1)}\left(\Theta_{L, h}\right)^{n}>0
$$

then $\kappa(L)=n$, in particular $X$ is Moishezon.

In fact, the lower bound (1.4) applied with $E=\mathscr{O}_{X}$ implies immediately that $h^{0}\left(X, L^{k}\right) \geqslant c k^{n}$, hence $\kappa(L)=n$. Now, if $X$ is a modification of $Y$, we have $M(Y) \simeq$ $M(X)$, so $a(X)=a(Y)$, and $Y$ has to be Moishezon.

\section{B. Cohomology estimates for nef line bundles}

On a projective algebraic manifold $X$, a line bundle $L$ is said to be nef if $L \cdot C \geqslant 0$ for every algebraic curve $C \subset X$. If $\omega$ is a given Kähler or Hermitian $(1,1)$-form on $X$, it can be shown (cf. [Dem90]) that $L$ is nef if and only if for every $\varepsilon>0$ there exists a smooth Hermitian metric $h_{\varepsilon}$ such that $\Theta_{L, h_{\varepsilon}} \geqslant-\varepsilon \omega$ on $X$; in fact, the latter property clearly implies

$$
L \cdot C=\int_{C} \Theta_{L, h_{\varepsilon}} \geqslant-\varepsilon \int_{C} \omega \Longrightarrow L \cdot C \geqslant 0
$$

for every curve $C$. Conversely, if $L \cdot C \geqslant 0$ for every curve $C$, the well-known Kleiman criterion (cf. [Har70]) implies that $k L+A$ is ample for every ample divisor $A$. Hence there exists a smooth Hermitian metric $h_{k}$ on $L$ such that

$$
\Theta_{k L+A}=k \Theta_{L, h_{k}}+\Theta_{A, h_{A}}>0 \Longrightarrow \Theta_{L, h_{k}} \geqslant-\frac{1}{k} \omega, \text { where } \omega=\Theta_{A, h_{A}}>0 .
$$

Therefore, one can introduce the following definition of nefness on an arbitrary compact complex manifold.

(2.9) Definition. Let $X$ be a compact complex manifold and $\omega$ a given smooth positive $(1,1)$-form on $X$. A line bundle $L \rightarrow X$ is said to be nef if for every $\varepsilon>0$ there exists a smooth Hermitian metric $h_{\varepsilon}$ on $L$ such that $\Theta_{L, h_{\varepsilon}} \geqslant-\varepsilon \omega$ everywhere on $X$.

(2.10) A consequence of holomorphic Morse inequalities. If $X$ is compact Kähler and $L$ is nef, for every holomorphic vector bundle $E$ on $X$ one has

$$
h^{q}(X, \mathscr{O}(E) \otimes \mathscr{O}(k L))=o\left(k^{n}\right) \quad \text { for all } q \geqslant 1 .
$$

Proof. Let $\omega$ be a Kähler metric. The nefness of $L$ implies that there exists a smooth Hermitian metric $h_{\varepsilon}$ on $L$ such that $\Theta_{L, h_{\varepsilon}} \geqslant-\varepsilon \omega$. On $X\left(L, h_{\varepsilon}, 1\right)$ we have exactly 
1 negative eigenvalue $\lambda_{1}$ which is belongs to $\left[-\varepsilon, 0\left[\right.\right.$ and the other ones $\lambda_{j}(j \geqslant 2)$ are positive. The product $\lambda_{1} \cdots \lambda_{n}$ satisfies $\left|\lambda_{1} \cdots \lambda_{n}\right| \leqslant \varepsilon \prod_{j \geqslant 2}\left(\lambda_{j}+\varepsilon\right)$, hence

$$
\frac{1}{n !}\left|\Theta_{L, h_{\varepsilon}}^{n}\right| \leqslant \frac{1}{(n-1) !} \varepsilon \omega \wedge\left(\Theta_{L, h_{\varepsilon}}+\varepsilon \omega\right)^{n-1} \quad \text { on } X\left(L, h_{\varepsilon}, 1\right) .
$$

By integrating, we find

$$
\int_{X\left(L, h_{\varepsilon}, 1\right)} \Theta_{L, h_{\varepsilon}}^{n} \leqslant n \varepsilon \int_{X} \omega \wedge\left(c_{1}(L)+\varepsilon \omega\right)^{n-1}
$$

and the result follows.

(2.11) Note. When $X$ is non Kähler, D. Popovici [Pop08] has announced bounds for the Monge-Ampère masses of $\Theta_{L, h_{\varepsilon}}$ which still imply the result, but the proof is much harder in that case. On the other hand, when $X$ is projective algebraic, an elementary hyperplane section argument and an induction on dimension easily implies the stronger upper bounds

$$
h^{q}(X, \mathscr{O}(E) \otimes \mathscr{O}(k L))=O\left(k^{n-q}\right) \quad \text { for all } q \geqslant 0
$$

Hint. By Serre duality, it is enough to show that

$$
h^{q}(X, \mathcal{O}(F) \otimes \mathscr{O}(-k L))=O\left(k^{q}\right) \quad \text { for every } q \geqslant 0
$$

and every holomorphic vector bundle $F$. Choose a very ample line bundle $A$ so big that $F^{\prime}=F^{*} \otimes \mathscr{O}(A)$ is Nakano positive, and apply the Nakano vanishing theorem and Serre duality to see that $H^{q}(X, \mathscr{O}(F) \otimes \mathscr{O}(-A) \otimes \mathscr{O}(-k L))=0$ for all $k$ and $q \geqslant 1$. Use the exact sequence $0 \rightarrow \mathscr{O}_{X}(-A) \rightarrow \mathscr{O}_{X} \rightarrow \mathscr{G}_{A} \rightarrow 0$, take the tensor product with $\mathscr{O}(F) \otimes \mathscr{O}(-k L)$ and apply induction.

It is unknown whether the accurate bound (2.12) holds true on a general compact complex manifold, even when $X$ is assumed to be Kähler.

\section{C. Distortion inequalities for asymptotic Fubini-Study metrics}

Another application of the heat kernel estimates is a generalization of G. Kempf's distortion inequalities ([Kem89], [Ji89]) to all projective algebraic manifolds. In this generality, the result was obtained by Th. Bouche [Bou90], and in less generality (but with somewhat stronger estimates) by G. Tian [Tia90].

Let $L$ be a positive Hermitian line bundle over a projective manifold $X$, equipped with a Hermitian metric $\omega$. Then $V_{k}=H^{0}\left(X, L^{k}\right)$ has a natural Hermitian metric given by the global $L^{2}$ norm of sections. For $k \geqslant k_{0}$ large enough, $\Phi_{k L}$ is an embedding and $L^{k}$ can be identified to the pull-back $\Phi_{k}^{*} O(1)$. We want to compare the original metric $|\bullet|$ of $L$ and the metric $|\cdot|_{\text {FS }}$ induced by the Fubini-Study metric of $O(1)$.

Let $\left(s_{1}, \ldots, s_{N}\right)$ be an orthonormal basis of $H^{0}\left(X, L^{k}\right)$. It is not difficult to check that

$$
|\xi|_{\mathrm{FS}}^{2}=\frac{|\xi|^{2}}{\left|s_{1}(x)\right|^{2}+\cdots+\left|s_{N}(x)\right|^{2}} \text { for } \xi \in L_{x}^{k}
$$


thus all that we need is to get an estimate of $\sum\left|s_{j}(x)\right|^{2}$. However, this sum is the contribution of the 0 eigenvalue in the heat kernel

$$
K_{t}^{k}(x, x)=\sum_{j=1}^{+\infty} e^{-2 t \lambda_{j}^{k}}\left|\psi_{j}(x)\right|^{2}
$$

associated to $\frac{2}{k} \square_{k}^{\prime \prime}$ in bidegree $(0,0)$. We observe that non zero eigenvalues $\lambda_{j}^{k}$ are also eigenvalues in bidegree $(0,1)$, since $\bar{\partial}$ is injective on the corresponding eigenspaces. The associated eigenfunctions are $\bar{\partial} \psi_{j} / \sqrt{k \lambda_{j}^{k}}$, for

$$
\left\|\bar{\partial} \psi_{j}\right\|^{2}=\left\langle\left\langle\Delta_{k}^{\prime \prime} \psi_{j}, \psi_{j}\right\rangle\right\rangle=k \lambda_{j}^{k}
$$

Thus the summation

$$
\sum_{j=1}^{+\infty} e^{-2 t \lambda_{j}^{k}}\left|\bar{\partial} \psi_{j}(x)\right|^{2}
$$

is bounded by the heat kernel in bidegree $(0,1)$, which is itself bounded by $k^{n} e^{-c t}$ with $c>0$ (note that $\alpha_{\complement}-\alpha_{J}-\sum\left|\alpha_{j}\right|<0$ on $X$ for $|J|=1$ ). Taking $t=k^{\varepsilon}$ with $\varepsilon$ small, one can check that all estimates remain uniformly valid and that the contribution of the non zero eigenfunctions in $K_{t}^{k}(x, x)$ becomes negligible in $C^{0}$ norm. Then theorem 1.23 shows that

$$
\sum\left|s_{j}(x)\right|^{2} \sim K_{t}^{k}(x, x) \sim k^{n}(2 \pi)^{-n}\left|\alpha_{1}(x) \cdots \alpha_{n}(x)\right|
$$

as $t=k^{\varepsilon} \rightarrow+\infty$. For $\xi \in L_{x}^{k}$ we get therefore the $C^{0}$ uniform estimate

$$
\frac{|\xi|^{2}}{|\xi|_{\mathrm{FS}}^{2}} \sim\left(\frac{k}{2 \pi}\right)^{n}\left|\alpha_{1}(x) \cdots \alpha_{n}(x)\right| \quad \text { as } \quad k \rightarrow+\infty .
$$

As a consequence, the Fubini-Study metric on $L$ induced by $\Phi_{k L}$ converges uniformly to the original metric. G. Tian [Tia90] proved that this last convergence statement holds in norm $C^{4}$. It is now known that there is in fact an asymptotic expansion in $1 / k$, and therefore $C^{\infty}$ convergence; this holds true even in the almost complex setting, see [BU00] and [SZ02].

\section{D. Algebraic counterparts of the holomorphic Morse inequalities}

One difficulty in the application of the analytic form of the inequalities is that the curvature integral is in general quite uneasy to compute, since it is neither a topological nor an algebraic invariant. However, the Morse inequalities can be reformulated in a more algebraic setting in which only algebraic invariants are involved. We give here two such reformulations - after they were found via analysis in [Dem94], F. Angelini [Ang96] gave a purely algebraic proof (see also [Siu93] and [Tra95] for related ideas).

(2.14) Theorem. Let $L=F-G$ be a holomorphic line bundle over a compact Kähler manifold $X$, where $F$ and $G$ are numerically effective line bundles. Then for every $q=0,1, \ldots, n=\operatorname{dim} X$, there is an asymptotic strong Morse inequality

$$
\sum_{0 \leqslant j \leqslant q}(-1)^{q-j} h^{j}(X, k L) \leqslant \frac{k^{n}}{n !} \sum_{0 \leqslant j \leqslant q}(-1)^{q-j}\left(\begin{array}{l}
n \\
j
\end{array}\right) F^{n-j} \cdot G^{j}+o\left(k^{n}\right) .
$$


Proof. By adding $\varepsilon$ times a Kähler metric $\omega$ to the curvature forms of $F$ and $G, \varepsilon>0$ one can write $\Theta_{L}=\tilde{\Theta}_{F, \varepsilon}-\tilde{\Theta}_{G, \varepsilon}$ where $\tilde{\Theta}_{F, \varepsilon}=\frac{i}{2 \pi} \Theta_{F}+\varepsilon \omega$ and $\tilde{\Theta}_{G, \varepsilon}=\frac{i}{2 \pi} \Theta_{G}+\varepsilon \omega$ are positive definite. Let $\lambda_{1} \geqslant \cdots \geqslant \lambda_{n}>0$ be the eigenvalues of $\tilde{\Theta}_{G, \varepsilon}$ with respect to $\tilde{\Theta}_{F, \varepsilon}$. Then the eigenvalues of $\frac{i}{2 \pi} \Theta_{L}$ with respect to $\tilde{\Theta}_{F, \varepsilon}$ are the real numbers $1-\lambda_{j}$ and the set $X(L, h, \leqslant q)$ is the set $\left\{\lambda_{q+1}<1\right\}$ of points $x \in X$ such that $\lambda_{q+1}(x)<1$. The strong Morse inequalities yield

$$
\sum_{0 \leqslant j \leqslant q}(-1)^{q-j} h^{j}(X, k L) \leqslant \frac{k^{n}}{n !} \int_{\left\{\lambda_{q+1}<1\right\}}(-1)^{q} \prod_{1 \leqslant j \leqslant n}\left(1-\lambda_{j}\right) \tilde{\Theta}_{F, \varepsilon}^{n}+o\left(k^{n}\right) .
$$

On the other hand we have

$$
\left(\begin{array}{c}
n \\
j
\end{array}\right) \tilde{\Theta}_{F, \varepsilon}^{n-j} \wedge \tilde{\Theta}_{G, \varepsilon}^{j}=\sigma_{n}^{j}(\lambda) \tilde{\Theta}_{F, \varepsilon}^{n}
$$

where $\sigma_{n}^{j}(\lambda)$ is the $j$-th elementary symmetric function in $\lambda_{1}, \ldots, \lambda_{n}$, hence

$$
\sum_{0 \leqslant j \leqslant q}(-1)^{q-j}\left(\begin{array}{c}
n \\
j
\end{array}\right) F^{n-j} \cdot G^{j}=\lim _{\varepsilon \rightarrow 0} \int_{X} \sum_{0 \leqslant j \leqslant q}(-1)^{q-j} \sigma_{n}^{j}(\lambda) \tilde{\Theta}_{F, \varepsilon}^{n} \cdot
$$

Thus, to prove the lemma, we only have to check that

$$
\sum_{0 \leqslant j \leqslant n}(-1)^{q-j} \sigma_{n}^{j}(\lambda)-\mathbb{1}_{\left\{\lambda_{q+1}<1\right\}}(-1)^{q} \prod_{1 \leqslant j \leqslant n}\left(1-\lambda_{j}\right) \geqslant 0
$$

for all $\lambda_{1} \geqslant \cdots \geqslant \lambda_{n} \geqslant 0$, where $\mathbb{1}_{\{\ldots\}}$ denotes the characteristic function of a set. This is easily done by induction on $n$ (just split apart the parameter $\lambda_{n}$ and write $\left.\sigma_{n}^{j}(\lambda)=\sigma_{n-1}^{j}(\lambda)+\sigma_{n-1}^{j-1}(\lambda) \lambda_{n}\right)$.

In the case $q=1$, we get an especially interesting lower bound (this bound has been observed and used by S. Trapani [Tra95] in a similar context).

(2.15) Consequence. $h^{0}(X, k L)-h^{1}(X, k L) \geqslant \frac{k^{n}}{n !}\left(F^{n}-n F^{n-1} \cdot G\right)-o\left(k^{n}\right)$. Therefore some multiple $k L$ has a section as soon as $F^{n}-n F^{n-1} \cdot G>0$.

(2.16) Remark. The weaker inequality

$$
h^{0}(X, k L) \geqslant \frac{k^{n}}{n !}\left(F^{n}-n F^{n-1} \cdot G\right)-o\left(k^{n}\right)
$$

is easy to prove if $X$ is projective algebraic. Indeed, by adding a small ample $\mathbb{Q}$-divisor to $F$ and $G$, we may assume that $F, G$ are ample. Let $m_{0} G$ be very ample and let $k^{\prime}$ be the smallest integer $\geqslant k / m_{0}$. Then $h^{0}(X, k L) \geqslant h^{0}\left(X, k F-k^{\prime} m_{0} G\right)$. We select $k^{\prime}$ smooth members $G_{j}, 1 \leqslant j \leqslant k^{\prime}$ in the linear system $\left|m_{0} G\right|$ and use the exact sequence

$$
0 \rightarrow H^{0}\left(X, k F-\sum G_{j}\right) \rightarrow H^{0}(X, k F) \rightarrow \bigoplus H^{0}\left(G_{j}, k F_{\mid G_{j}}\right)
$$


Kodaira's vanishing theorem yields $H^{q}(X, k F)=0$ and $H^{q}\left(G_{j}, k F_{\mid G_{j}}\right)=0$ for $q \geqslant 1$ and $k \geqslant k_{0}$. By the exact sequence combined with Riemann-Roch, we get

$$
\begin{aligned}
h^{0}(X, k L) & \geqslant h^{0}\left(X, k F-\sum G_{j}\right) \\
& \geqslant \frac{k^{n}}{n !} F^{n}-O\left(k^{n-1}\right)-\sum\left(\frac{k^{n-1}}{(n-1) !} F^{n-1} \cdot G_{j}-O\left(k^{n-2}\right)\right) \\
& \geqslant \frac{k^{n}}{n !}\left(F^{n}-n \frac{k^{\prime} m_{0}}{k} F^{n-1} \cdot G\right)-O\left(k^{n-1}\right) \\
& \geqslant \frac{k^{n}}{n !}\left(F^{n}-n F^{n-1} \cdot G\right)-O\left(k^{n-1}\right) .
\end{aligned}
$$

(This simple proof is due to F. Catanese.)

(2.17) Corollary. Suppose that $F$ and $G$ are nef and that $F$ is big. Some multiple of $m F-G$ has a section as soon as

$$
m>n \frac{F^{n-1} \cdot G}{F^{n}}
$$

In the last condition, the factor $n$ is sharp: this is easily seen by taking $X=\mathbb{P}_{1}^{n}$ and $F=\mathscr{G}(a, \ldots, a)$ and $G=\mathscr{O}\left(b_{1}, \ldots, b_{n}\right)$ over $\mathbb{P}_{1}^{n} ;$ the condition of the corollary is then $m>\sum b_{j} / a$, whereas $k(m F-G)$ has a section if and only if $m \geqslant \sup b_{j} / a$; this shows that we cannot replace $n$ by $n(1-\varepsilon)$.

\section{Morse inequalities on $q$-convex varieties}

Thierry Bouche [Bou89] has obtained an extension of holomorphic Morse inequalities to the case of strongly $q$-convex manifolds. We explain here the main ideas involved.

A complex (non compact) manifold $X$ of dimension $n$ is strongly $q$-convex in the sense of Andreotti and Grauert [AG62] if there exists a $C^{\infty}$ exhaustion function $\psi$ on $X$ such that $i \partial \bar{\partial} \psi$ has at least $n-q+1$ positive eigenvalues outside a compact subset of $X$. In this case, the Andreotti-Grauert theorem shows that all cohomology groups $H^{m}(X, \mathscr{F})$ with values in a coherent analytic sheaf are finite dimensional for $m \geqslant q$.

(3.1) Theorem. Let $L, E$ be holomorphic vector bundles over $X$ with $\operatorname{rank} L=1$, rank $E=r$. Assume that $X$ is strongly $q$-convex and that $L$ has a Hermitian metric $h$ for which $\Theta_{L, h}$ has at least $n-p+1$ nonnegative eigenvalues outside a compact subset $K \subset X$. Then for all $m \geqslant p+q-1$ the following strong Morse inequalities hold:

$$
\sum_{\ell=m}^{n}(-1)^{\ell-m} \operatorname{dim} H^{\ell}\left(X, E \otimes L^{k}\right) \leqslant r \frac{k^{n}}{n !} \int_{X(L, h, \geqslant m)}(-1)^{m} \Theta_{L, h}^{n}+o\left(k^{n}\right) .
$$

Proof. For every $c \in \mathbb{R}$, we consider the sublevel sets

$$
X_{c}=\{x \in X ; \psi(x)<c\} .
$$


Select $c_{0}$ such that $i \partial \bar{\partial} \psi$ has $n-q+1$ positive eigenvalues on $X \backslash X_{c}$. One can choose a Hermitian metric $\omega_{0}$ on $X$ in such a way that the eigenvalues $\gamma_{1}^{0} \leqslant \cdots \leqslant \gamma_{n}^{0}$ of $i \partial \bar{\partial} \psi$ with respect to $\omega_{0}$ satisfy

$$
-\frac{1}{n} \leqslant \gamma_{1}^{0} \leqslant \cdots \leqslant \gamma_{q-1}^{0} \leqslant 1 \quad \text { and } \quad \gamma_{q}^{0}=\cdots=\gamma_{n}^{0}=1 \text { on } X \backslash X_{c_{0}}
$$

this can be achieved by taking $\omega_{0}$ equal to $i \partial \bar{\partial} \psi$ on a $C^{\infty}$ subbundle of $T_{X}$ of rank $n-q+1$ on which $i \partial \bar{\partial} \psi$ is positive, and $\omega_{0}$ very large on the orthogonal complement. We set $\omega=e^{\rho} \omega_{0}$ where $\rho$ is a function increasing so fast at infinity that $\omega$ will be complete.

More important, we multiply the metric of $L$ by a weight $e^{-\chi \circ \psi}$ where $\chi$ is a convex increasing function. The resulting Hermitian line bundle is denoted $\left(L_{\chi}, h_{\chi}\right)$. For any $(0, m)$ form $u$ with values in $E \otimes L^{k}$, viewed as an $(n, m)$ form with values in $E \otimes L^{k} \otimes$ $\Lambda^{n} T_{X}$, the Bochner-Kodaira-Nakano formula implies an inequality

$$
\left.\left\langle\left\langle\Delta_{k}^{\prime \prime} u, u\right\rangle\right\rangle \geqslant \int_{X} k\left\langle\left[i \theta_{L_{\chi}, h_{\chi}}\right), \Lambda\right] u, u\right\rangle+\langle W u, u\rangle
$$

where $W$ depends only on the curvature of $E \otimes \Lambda^{n} T_{X}$ and the torsion of $\omega$. By the formulas of $\S 1$.C, we have

$$
\left.\left\langle\left[i \theta_{L_{\chi}, h_{\chi}}\right), \Lambda\right] u, u\right\rangle \geqslant\left(\alpha_{1}+\cdots+\alpha_{m}\right)|u|^{2}
$$

where $\alpha_{1} \leqslant \cdots \leqslant \alpha_{n}$ are the eigenvalues of

$$
i \theta_{L_{\chi}, h_{\chi}}=i \theta_{L, h}+i \partial \bar{\partial}(\chi \circ \psi) \geqslant i \theta_{L, h}+\left(\chi^{\prime} \circ \psi\right) i \partial \bar{\partial} \psi
$$

If $\beta$ is the lowest eigenvalue of $i \theta_{L, h}$ with respect to $\omega$, we find

$$
\begin{aligned}
\alpha_{j} & \geqslant \beta+\left(\chi^{\prime} \circ \psi\right) \gamma_{j}^{0} / e^{\rho} \\
\alpha_{1}+\cdots+\alpha_{m} & \geqslant m \beta+\left(\chi^{\prime} \circ \psi\right)\left(\gamma_{1}^{0}+\cdots+\gamma_{m}^{0}\right) / e^{\rho}
\end{aligned}
$$

and by (3.2) we get for all $m \geqslant q$ :

$$
\alpha_{1}+\cdots+\alpha_{m} \geqslant m \beta+\frac{1}{n} e^{-\rho} \chi^{\prime} \circ \psi \text { on } X \backslash X_{c_{0}}
$$

It follows that one can choose $\chi$ increasing very fast in such a way that the Bochner inequality becomes

$$
\left\langle\Delta_{k}^{\prime \prime} u, u\right\rangle \geqslant k \int_{X \backslash X_{c_{0}}} A(x)|u(x)|^{2}-C_{1} \int_{X}|u(x)|^{2}
$$

where $A \geqslant 1$ is a function tending to $+\infty$ at infinity on $X$ and $C_{1} \geqslant 0$. Now, Rellich's lemma easily shows that $\Delta_{k}^{\prime \prime}$ has a compact resolvent. Hence the spectrum of $\Delta_{k}^{\prime \prime}$ is discrete and its eigenspaces are finite dimensional. Standard arguments also show the following : 
(3.4) Lemma. When $\chi$ increases sufficiently fast at infinity, the space $\mathscr{H}^{m}\left(X, L_{\chi}^{k} \otimes E\right)$ of $L^{2}$-harmonic forms of bidegree $(0, m)$ for $\Delta_{k}^{\prime \prime}$ is isomorphic to the cohomology group $H^{m}\left(X, E \otimes L^{k}\right)$ for all $k \in \mathbb{N}$ and $m \geqslant q$.

For a domain $\Omega \subset \subset X$, we consider the quadratic form

$$
Q_{\Omega}^{k, m}(u)=\frac{1}{k} \int_{\Omega}\left|\bar{\partial}_{k} u\right|^{2}+\left|\bar{\partial}_{k}^{*} u\right|^{2}
$$

with Dirichlet boundary conditions on $\partial \Omega$. We denote by $\mathscr{H}_{\leqslant \lambda, \Omega}^{k, m}$ the direct sum of all eigenspaces of $Q_{\Omega}^{k, m}$ corresponding to eigenvalues $\leqslant \lambda$ (i.e. $\leqslant k \lambda$ for $\Delta_{k}^{\prime \prime}$ ).

(3.5) Lemma. For every $\lambda \geqslant 0$ and $\varepsilon>0$, there exists a domain $\Omega \subset \subset X$ and an integer $k_{0}$ such that

$$
\operatorname{dim} \mathscr{H}_{\leqslant \lambda, \Omega}^{k, m} \leqslant \operatorname{dim} \mathscr{H}_{\leqslant \lambda, X}^{k, m} \leqslant \operatorname{dim} \mathscr{H}_{\leqslant \lambda+\varepsilon, \Omega}^{k, m} \text { for } k \geqslant k_{0} .
$$

Proof. The left hand inequality is a straightforward consequence of the minimax principle, because the domain of the global quadratic form $Q_{\Omega}^{k, m}$ is contained in the domain of $Q_{X}^{k, m}$.

For the other inequality, let $u \in \mathscr{H}_{\leqslant \lambda, X}^{k, m}$. Then (3.3) gives

$$
k \int_{X \backslash X_{c_{0}}} A|u|^{2}-C_{1} \int_{X_{c_{0}}}|u|^{2} \leqslant k \lambda \int_{X}|u|^{2} .
$$

Choose $c_{2}>c_{1}>c_{0}$ so that $A(x) \geqslant a$ on $X \backslash X_{c_{1}}$ and a cut-off function $\varphi$ with compact support in $X_{c_{2}}$ such that $0 \leqslant \varphi \leqslant 1$ and $\varphi=1$ on $X_{c_{1}}$. Then we find

$$
\int_{X \backslash X_{c_{1}}}|u|^{2} \leqslant \frac{C_{1}+k \lambda}{k a} \int_{X}|u|^{2} .
$$

For a large enough, we get $\int_{X \backslash X_{c_{1}}}|u|^{2} \leqslant \varepsilon\|u\|^{2}$. Set $\Omega=X_{c_{2}}$. Then

$$
\begin{aligned}
Q_{\Omega}^{k, m}(\varphi u) & \left.=\frac{1}{k} \int_{\Omega}\left|\bar{\partial} \varphi \wedge u+\varphi \bar{\partial}_{k} u\right|^{2}+\mid \varphi \bar{\partial}_{k}^{*} u-\partial \varphi\right\lrcorner\left. u\right|^{2} \\
& \leqslant(1+\varepsilon) Q_{X}^{k, m}(u)+\frac{C_{2}}{k}\left(1+\frac{1}{\varepsilon}\right)\|u\|^{2} \\
& \leqslant(1+\varepsilon)\left(\lambda+\frac{C_{2}}{k \varepsilon}\right)\|u\|^{2}
\end{aligned}
$$

As $\|\varphi u\|^{2} \geqslant \int_{X_{c_{1}}}|u|^{2} \geqslant(1-\varepsilon)\|u\|^{2}$, we infer

$$
Q_{\Omega}^{k, m}(\varphi u) \leqslant \frac{1+\varepsilon}{1-\varepsilon}\left(\lambda+\frac{C_{2}}{k \varepsilon}\right)\|\varphi u\|^{2}
$$

If $\varepsilon$ is replaced by a suitable smaller number and $k$ taken large enough, we obtain $Q_{\Omega}^{k, m}(v) \leqslant(\lambda+\varepsilon)\|v\|^{2}$ for all $v \in \varphi \mathscr{H}_{\leqslant \lambda, X}^{k, m}$. Then the right hand inequality in lemma 3.5 follows by the minimax principle. 
Now, Corollary 1.17 easily computes the counting function $N_{\Omega}^{k, m}$ for the eigenvalues :

$$
\left.\lim _{\lambda \rightarrow 0_{+}} \lim _{k \rightarrow+\infty} k^{-n} N_{\Omega}^{k, m}(\lambda)=\frac{r}{n !} \int_{X\left(L_{\chi}, h_{\chi}, m\right)}(-1)^{m}\left(\frac{i}{2 \pi} \theta_{L_{\chi}, h_{\chi}}\right)\right)^{n} .
$$

Applying this to the Witten complex $\mathscr{H}_{\leqslant \lambda, X}^{k, \bullet}$, we easily infer the inequality of theorem 3.1 , except that $c(L)$ is replaced by $c\left(L_{\chi}\right)$. However, up to now, the inequality is valid for all $m \geqslant q$. Take the convex function $\chi$ equal to 0 on $\left.]-\infty, c_{0}\right]$. Then

$$
\Theta_{L_{\chi}, h_{\chi}}=\frac{i}{2 \pi} \theta_{L_{\chi}, h_{\chi}}=\Theta_{L, h}+\frac{i}{2 \pi} \partial \bar{\partial}(\chi \circ \psi)
$$

coincides with $\Theta_{L, h}$ on $X_{c_{0}}$ and has at most $(p-1)+(q-1)$ negative eigenvalues on $X \backslash X_{c_{0}}$. Hence $X\left(L_{\chi}, h_{\chi}, m\right)=X(L, h, m)$ for $m \geqslant p+q-1$ and $\Theta_{L_{\chi}, h_{\chi}}=\Theta_{L, h}$ on these sets. Theorem 3.1 is proved.

As a corollary, one obtains a general a priori estimate for the Monge-Ampère operator $(i \partial \bar{\partial})^{n}$ on $q$-convex manifolds.

(3.6) Corollary: calculus inequalities. Let $X$ be a strongly $q$-convex manifold and $\varphi$ a $C^{\infty}$ function on $X$, weakly $p$-convex outside a compact subset of $X$. For $\ell=0,1, \ldots, n$, let $G_{\ell}$ be the open set of points where $i \partial \bar{\partial} \varphi$ is non degenerate and admits $\ell$ negative eigenvalues. Then for all $m \geqslant p=q-1$

$$
\sum_{\ell=m}^{n} \int_{G_{\ell}}(i \partial \bar{\partial} \varphi)^{m} \text { has the sign of }(-1)^{m} \text {. }
$$

This result has been first obtained by Y.T. Siu [Siu90] for $q$-convex domains in a Stein manifold. At that time, the $q$-convex case of the inequalities was not yet available and Siu had to rely on a rather sophisticated approximation argument of Stein manifolds by algebraic varieties ; the proof could then be reduced to the compact case.

The general statement given above is in fact a direct consequence of Theorem 3.1 : take for $L$ the trivial bundle $L=\mathscr{\sigma}_{X}$ equipped with the metric defined by the weight $e^{-\varphi}$ and $E=\mathscr{G}_{X}$. Since $H^{m}\left(X, L^{k}\right)=H^{m}\left(X, \mathscr{O}_{X}\right)$ is independent of $k$ and finite dimensional, Theorem 3.1 implies

$$
k^{n} \sum_{\ell=m}^{n} \int_{G_{\ell}}(-1)^{m}(i \partial \bar{\partial} \varphi)^{n} \geqslant \text { constant }-o\left(k^{n}\right)
$$

for all $k \geqslant k_{0}$ and $m \geqslant p+q-1$, whence the result. 


\section{Part II}

\section{Approximation of currents and intersection theory}

\section{Introduction}

Many concepts described in this chapter (e.g. pseudo-effectivity) are quite general and make sense on an arbitrary compact complex manifold $X$ - no projective or Kähler assumption is needed. In this general context, it is better to work with $\partial \bar{\partial}$-cohomology classes instead of De Rham cohomology classes: we define the Bott-Chern cohomology of $X$ to be

$$
H_{\mathrm{BC}}^{p, q}(X, \mathbb{C})=\{d \text {-closed }(p, q) \text {-forms }\} /\{\partial \bar{\partial} \text {-exact }(p, q) \text {-forms }\} .
$$

It is easily shown that these cohomology groups are finite dimensional and can be computed either with spaces of smooth forms or with currents; in fact, they can be computed by certain complexes of sheaves of forms or currents that both provide fine resolutions of the same sheaves of holomorphic or anti-holomorphic forms. Our statement therefore follows formally from general results of sheaf theory. Also, finiteness can be obtained by the usual Cartan-Serre proof based on Montel's theorem for Čech cohomology. In both cases, the quotient topology of $H_{\mathrm{BC}}^{p, q}(X, \mathbb{C})$ induced by the Fréchet topology of smooth forms or by the weak topology of currents is Hausdorff. Clearly, $H_{\mathrm{BC}}^{\bullet}(X, \mathbb{C})$ is a bigraded algebra, and it is trivial by definition that there are always canonical morphisms

$$
H_{\mathrm{BC}}^{p, q}(X, \mathbb{C}) \rightarrow H_{\frac{p}{\partial}}^{p, q}(X, \mathbb{C}), \quad \bigoplus_{p+q=k} H_{\mathrm{BC}}^{p, q}(X, \mathbb{C}) \rightarrow H_{\mathrm{DR}}^{k}(X, \mathbb{C}) .
$$

By Hodge decomposition and by the well-known $\partial \bar{\partial}$-lemma of Kähler geometry, these morphisms are isomorphisms when $X$ is Kähler; especially, we get a canonical algebra isomorphism

$$
H_{\mathrm{DR}}^{\bullet}(X, \mathbb{C}) \simeq \bigoplus_{p, q} H_{\bar{\partial}}^{p, q}(X, \mathbb{C}) \quad \text { if } X \text { is Kähler. }
$$

We will see in Section 5 (Remark 5.15) that this is true more generally if $X$ is in the Fujiki class $\mathscr{C}$, i.e., the class of manifolds bimeromorphic to Kähler manifolds.

\section{Pseudo-effective line bundles and singular Hermitian metrics}

Let $L$ be a holomorphic line bundle on a compact complex manifold $X$. It is important for many applications to allow singular Hermitian metrics. 
(1.1) Definition. A singular Hermitian metric $h$ on $L$ is a Hermitian metric such that, for any trivialisation $L_{\mid U} \simeq U \times \mathbb{C}$, the metric is given by $h=e^{-\varphi}, \varphi \in L_{\mathrm{loc}}^{1}(U)$.

The curvature tensor

$$
\Theta_{L, h}=\frac{i}{2 \pi} \partial \bar{\partial} \varphi=-\frac{i}{2 \pi} \partial \bar{\partial} \log h
$$

can then be computed in the sense of distributions, and defines in this way a (global) closed $(1,1)$-current on $X$. It defines a (real) cohomology class $\left\{\Theta_{L, h}\right\} \in H_{\mathrm{BC}}^{1,1}(X, \mathbb{C})$ which is mapped to the first Chern class $c_{1}(L)$ by the canonical morphisms $(0.2)$. We will therefore still denote this Bott-Chern class by $c_{1}(L)$. The positive case is of special interest.

(1.3) Definition. We say that $L$ pseudo-effective if $c_{1}(L) \in H_{\mathrm{BC}}^{1,1}(X, \mathbb{C})$ is the cohomology class of some closed positive current $T$, i.e. if $L$ can be equipped with a singular Hermitian metric $h$ with $T=\Theta_{L, h} \geqslant 0$ as a current, in other words, if the weight functions $\varphi$ can be chosen to be plurisubharmonic on each trivialization open set $U$.

The locus where $h$ has singularities turns out to be extremely important. One way is to introduce multiplier ideal sheaves following A. Nadel [Nad89]. The main idea actually goes back to the fundamental works of Bombieri [Bom70] and H. Skoda [Sko72a].

(1.4) Definition. Let $\varphi$ be a psh (plurisubharmonic) function on an open subset $\Omega \subset X$. To $\varphi$ we associate the ideal subsheaf $\mathscr{H}(\varphi) \subset \mathscr{G}_{\Omega}$ of germs of holomorphic functions $f \in \mathscr{G}_{\Omega, x}$ such that $|f|^{2} e^{-\varphi}$ is integrable with respect to the Lebesgue measure in some local coordinates near $x$.

The zero variety $V(\mathscr{F}(\varphi))$ is thus the set of points in a neighborhood of which $e^{-\varphi}$ is non integrable. The following result implies that this is always an analytic set.

(1.5) Proposition ([Nad89]). For any psh function $\varphi$ on $\Omega \subset X$, the sheaf $\mathscr{J}(\varphi)$ is a coherent sheaf of ideals over $\Omega$. Moreover, if $\Omega$ is a bounded Stein open set, the sheaf $\mathscr{F}(\varphi)$ is generated by any Hilbert basis of the $L^{2}$ space $\mathscr{H}^{2}(\Omega, \varphi)$ of holomorphic functions $f$ on $\Omega$ such that $\int_{\Omega}|f|^{2} e^{-\varphi} d \lambda<+\infty$.

Proof. Since the result is local, we may assume that $\Omega$ is a bounded pseudoconvex open set in $\mathbb{C}^{n}$. By the strong noetherian property of coherent sheaves, the family of sheaves generated by finite subsets of $\mathscr{H}^{2}(\Omega, \varphi)$ has a maximal element on each compact subset of $\Omega$, hence $\mathscr{H}^{2}(\Omega, \varphi)$ generates a coherent ideal sheaf $\mathscr{J} \subset \mathscr{O}_{\Omega}$. It is clear that $\mathscr{J} \subset \mathscr{F}(\varphi)$; in order to prove the equality, we need only check that $\mathscr{J}_{x}+\mathscr{I}(\varphi)_{x} \cap \mathfrak{m}_{\Omega, x}^{s+1}=\mathscr{J}(\varphi)_{x}$ for every integer $s$, in view of the Krull lemma. Let $f \in \mathscr{F}(\varphi)_{x}$ be defined in a neighborhood $V$ of $x$ and let $\theta$ be a cut-off function with support in $V$ such that $\theta=1$ in a neighborhood of $x$. We solve the equation $\bar{\partial} u=g:=\bar{\partial}(\theta f)$ by means of Hörmander's $L^{2}$ estimates [Hör65, AV65], applied with the strictly psh weight

$$
\widetilde{\varphi}(z)=\varphi(z)+(n+s) \log |z-x|^{2}+|z|^{2} .
$$

We get a solution $u$ such that $\int_{\Omega}|u|^{2} e^{-\varphi}|z-x|^{-2(n+s)} d \lambda<\infty$, thus $F=\theta f-u$ is holomorphic, $F \in \mathscr{H}^{2}(\Omega, \varphi)$ and $f_{x}-F_{x}=u_{x} \in \mathscr{F}(\varphi)_{x} \cap \mathfrak{m}_{\Omega, x}^{s+1}$. This proves the coherence. 
Now, $\mathscr{F}$ is generated by any Hilbert basis of $\mathscr{H}^{2}(\Omega, \varphi)$, because it is well-known that the space of sections of any coherent sheaf is a Fréchet space, therefore closed under local $L^{2}$ convergence.

Another important way of measuring singularities is via Lelong numbers - a natural generalization of the concept of multiplicity to psh functions. Recall that the Lelong number of a function $\varphi \in \operatorname{Psh}(\Omega)$ at a point $x_{0}$ is defined to be

$$
\nu\left(\varphi, x_{0}\right)=\liminf _{z \rightarrow x_{0}} \frac{\varphi(z)}{\log \left|z-x_{0}\right|}=\lim _{r \rightarrow 0_{+}} \frac{\sup _{B\left(x_{0}, r\right)} \varphi}{\log r} .
$$

In particular, if $\varphi=\log |f|$ with $f \in \mathscr{O}(\Omega)$, then $\nu\left(\varphi, x_{0}\right)$ is equal to the vanishing order

$$
\operatorname{ord}_{x_{0}}(f)=\sup \left\{k \in \mathbb{N} ; D^{\alpha} f\left(x_{0}\right)=0, \forall|\alpha|<k\right\} .
$$

The link with multiplier ideal sheaves is provided by the following standard result due to Skoda [Sko72a].

(1.7) Lemma. Let $\varphi$ be a psh function on an open set $\Omega$ and let $x \in \Omega$.

(a) If $\nu(\varphi, x)<2$, then $e^{-\varphi}$ is Lebesgue integrable on a neighborhood of $x$, in particular $\mathscr{J}(\varphi)_{x}=\mathscr{G}_{\Omega, x}$.

(b) More generally, if $\nu(\varphi, x) \geqslant 2(n+s)$ for some integer $s \geqslant 0$, then

$$
e^{-\varphi} \geqslant c|z-x|^{-2 n-2 s}, \quad c>0
$$

in a neighborhood of $x$, and $\mathscr{F}(\varphi)_{x} \subset \mathfrak{m}_{\Omega, x}^{s+1}$, where $\mathfrak{m}_{\Omega, x}$ is the maximal ideal of $\mathscr{O}_{\Omega, x}$. In particular $e^{-\varphi}$ is non integrable at $x$ if $\nu(\varphi, x) \geqslant 2 n$.

(c) The zero variety $V(\mathscr{I}(\varphi))$ of $\mathscr{J}(\varphi)$ satisfies

$$
V_{2 n}(\varphi) \subset V(\mathscr{F}(\varphi)) \subset E_{2}(\varphi)
$$

where $E_{c}(\varphi)=\{x \in X ; \nu(\varphi, x) \geqslant c\}$ is the c-upperlevel set of Lelong numbers of $\varphi$.

The only non trivial part is 1.7 (a); the proof relies on the Bochner-Martinelli representation formula for $T=\frac{i}{\pi} \partial \bar{\partial} \varphi$ (see [Sko72a]). One should observe that 1.7 (a) (resp. (b)) is optimal, as one can see by taking $\varphi(z)=\lambda \log \left|z_{1}\right|$, resp. $\varphi(z)=\lambda \log |z|$, on $\Omega=\mathbb{C}^{n}$.

\section{Hermitian metrics with minimal singularities and analytic Zariski decomposition}

We show here by a general "abstract" method that a pseudo-effective line bundle always has a Hermitian metric $h_{\text {min }}$ with minimal singularities among those with nonnegative curvature $\Theta_{L, h} \geqslant 0$ in the sense of currents. The following definition was introduced in [DPS01].

(2.1) Definition. Let $L$ be a pseudo-effective line bundle on a compact complex manifold $X$. Consider two Hermitian metrics $h_{1}, h_{2}$ on $L$ with curvature $\Theta_{L, h_{j}} \geqslant 0$ in the sense of currents. 
(a) We will write $h_{1} \preccurlyeq h_{2}$, and say that $h_{1}$ is less singular than $h_{2}$, if there exists a constant $C>0$ such that $h_{1} \leqslant C h_{2}$.

(b) We will write $h_{1} \sim h_{2}$, and say that $h_{1}, h_{2}$ are equivalent with respect to singularities, if there exists a constant $C>0$ such that $C^{-1} h_{2} \leqslant h_{1} \leqslant C h_{2}$.

Of course $h_{1} \preccurlyeq h_{2}$ if and only if the associated weights in suitable trivializations locally satisfy $\varphi_{2} \leqslant \varphi_{1}+C$. This implies in particular $\nu\left(\varphi_{1}, x\right) \leqslant \nu\left(\varphi_{2}, x\right)$ at each point. The above definition is motivated by the following observation.

(2.2) Theorem. For every pseudo-effective line bundle $L$ over a compact complex manifold $X$, there exists up to equivalence of singularities a unique class of Hermitian metrics $h$ with minimal singularities such that $\Theta_{L, h} \geqslant 0$.

Proof. The proof is almost trivial. We fix once for all a smooth metric $h_{\infty}$ (whose curvature is of random sign and signature), and we write singular metrics of $L$ under the form $h=h_{\infty} e^{-\psi}$. The condition $\Theta_{L, h} \geqslant 0$ is equivalent to $\frac{i}{2 \pi} \partial \bar{\partial} \psi \geqslant-u$ where $u=\Theta_{L, h_{\infty}}$. This condition implies that $\psi$ is plurisubharmonic up to the addition of the weight $\varphi_{\infty}$ of $h_{\infty}$, and therefore locally bounded from above. Since we are concerned with metrics only up to equivalence of singularities, it is always possible to adjust $\psi$ by a constant in such a way that $\sup _{X} \psi=0$. We now set

$$
h_{\min }=h_{\infty} e^{-\psi_{\min }}, \quad \psi_{\min }(x)=\sup _{\psi} \psi(x)
$$

where the supremum is extended to all functions $\psi$ such that $\sup _{X} \psi=0$ and $\frac{i}{2 \pi} \partial \bar{\partial} \psi \geqslant$ $-u$. By standard results on plurisubharmonic functions (see Lelong [Lel69]), $\psi_{\min }$ still satisfies $\frac{i}{2 \pi} \partial \bar{\partial} \psi_{\min } \geqslant-u$ (i.e. the weight $\varphi_{\infty}+\psi_{\min }$ of $h_{\min }$ is plurisubharmonic), and $h_{\min }$ is obviously the metric with minimal singularities that we were looking for. [In principle one should take the upper semicontinuous regularization $\psi_{\min }^{*}$ of $\psi_{\min }$ to really get a plurisubharmonic weight, but since $\psi_{\min }^{*}$ also participates to the upper envelope, we obtain here $\psi_{\min }=\psi_{\min }^{*}$ automatically].

(2.3) Remark. In general, the supremum $\psi=\sup _{j \in I} \psi_{j}$ of a locally dominated family of plurisubharmonic functions $\psi_{j}$ is not plurisubharmonic strictly speaking, but its "up-

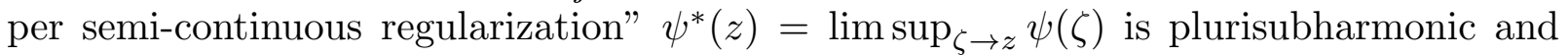
coincides almost everywhere with $\psi$, with $\psi^{*} \geqslant \psi$. However, in the context of $(2.3), \psi^{*}$ still satisfies $\psi^{*} \leqslant 0$ and $\frac{i}{2 \pi} \partial \bar{\partial} \psi \geqslant-u$, hence $\psi^{*}$ participates to the upper envelope. As a consequence, we have $\psi^{*} \leqslant \psi$ and thus $\psi=\psi^{*}$ is indeed plurisubharmonic. Under a strict positivity assumption, namely if $L$ is a big line bundle (i.e. the curvature can be taken to be strictly positive in the sense of currents, see Definition (3.3 d) and Theorem $(3.4 \mathrm{~b})$, then $h_{\min }$ can be shown to possess some regularity properties. The reader may consult [BmD09] for a rather general (but certainly non trivial) proof that $\psi_{\text {min }}$ possesses locally bounded second derivatives $\partial^{2} \psi_{\min } / \partial z_{j} \partial \bar{z}_{k}$ outside an analytic set $Z \subset X$; in other words, $\Theta_{L, h_{\min }}$ has locally bounded coefficients on $X \backslash Z$.

(2.4) Definition. Let $L$ be a pseudo-effective line bundle. If $h$ is a singular Hermitian metric such that $\Theta_{L, h} \geqslant 0$ and

$$
H^{0}\left(X, m L \otimes \mathscr{F}\left(h^{\otimes m}\right)\right) \simeq H^{0}(X, m L) \quad \text { for all } m \geqslant 0,
$$


we say that $h$ is an analytic Zariski decomposition of $L$.

In other words, we require that $h$ has singularities so mild that the vanishing conditions prescribed by the multiplier ideal sheaves $\mathscr{F}\left(h^{\otimes m}\right)$ do not kill any sections of $L$ and its multiples.

(2.5) Exercise. A special case is when there is an isomorphism $p L=A+E$ where $A$ and $E$ are effective divisors such that $H^{0}(X, m p L)=H^{0}(X, m A)$ for all $m$ and $\mathscr{O}(A)$ is generated by sections. Then $A$ possesses a smooth Hermitian metric $h_{A}$, and this metric defines a singular Hermitian metric $h$ on $L$ with poles $\frac{1}{p} E$ and curvature $\frac{1}{p} \Theta_{A, h_{A}}+\frac{1}{p}[E]$. Show that this metric $h$ is an analytic Zariski decomposition.

Note: when $X$ projective and there is a decomposition $p L=A+E$ with $A$ nef (see (I 2.9)), $E$ effective and $H^{0}(X, m p L)=H^{0}(X, m A)$ for all $m$, one says that the $\mathbb{Q}$ divisor equality $L=\frac{1}{p} A+\frac{1}{p} E$ is an algebraic Zariski decomposition of $L$. It can be shown that Zariski decompositions exist in dimension 2, but in higher dimension they do not exist in general.

(2.6) Theorem. The metric $h_{\min }$ with minimal singularities provides an analytic Zariski decomposition.

It follows that an analytic Zariski decomposition always exists (while algebraic decompositions do not exist in general, especially in dimension 3 and more).

Proof. Let $\sigma \in H^{0}(X, m L)$ be any section. Then we get a singular metric $h$ on $L$ by putting $|\xi|_{h}=\left|\xi / \sigma(x)^{1 / m}\right|$ for $\xi \in L_{x}$, and it is clear that $|\sigma|_{h^{m}}=1$ for this metric. Hence $\sigma \in H^{0}\left(X, m L \otimes \mathscr{F}\left(h^{\otimes m}\right)\right)$, and a fortiori $\sigma \in H^{0}\left(X, m L \otimes \mathscr{F}\left(h_{\min }^{\otimes m}\right)\right)$ since $h_{\min }$ is less singular than $h$.

\section{Description of the positive cones (Kähler and projective cases)}

Let us recall that an integral cohomology class in $H^{2}(X, \mathbb{Z})$ is the first Chern class of a holomorphic (or algebraic) line bundle if and only if it lies in the Neron-Severi group

$$
\mathrm{NS}(X)=\operatorname{Ker}\left(H^{2}(X, \mathbb{Z}) \rightarrow H^{2}\left(X, \mathscr{O}_{X}\right)\right)
$$

(this fact is just an elementary consequence of the exponential exact sequence $0 \rightarrow \mathbb{Z} \rightarrow \mathscr{O} \rightarrow \mathscr{O}^{*} \rightarrow 0$ ). If $X$ is compact Kähler, as we will suppose from now on in this section, this is the same as saying that the class is of type $(1,1)$ with respect to Hodge decomposition.

Let us consider the real vector space $\mathrm{NS}_{\mathbb{R}}(X)=\mathrm{NS}(X) \otimes_{\mathbb{Z}} \mathbb{R}$, which can be viewed as a subspace of the space $H^{1,1}(X, \mathbb{R})$ of real $(1,1)$ cohomology classes. Its dimension is by definition the Picard number

$$
\rho(X)=\operatorname{rank}_{\mathbb{Z}} \mathrm{NS}(X)=\operatorname{dim}_{\mathbb{R}} \mathrm{NS}_{\mathbb{R}}(X) .
$$

We thus have $0 \leqslant \rho(X) \leqslant h^{1,1}(X)$, and the example of complex tori shows that all intermediate values can occur when $n=\operatorname{dim} X \geqslant 2$. 
The positivity concepts for line bundles considered in section I 2.B and II 1 possess in fact natural generalizations to $(1,1)$ classes which are not necessarily integral or rational and this works at least in the category of compact Kähler manifolds (in fact, by using Bott-Chern cohomology, one could even extend these concepts to arbitrary compact complex manifolds).

(3.3) Definition. Let $(X, \omega)$ be a compact Kähler manifold.

(a) The Kähler cone is the set $\mathscr{K} \subset H^{1,1}(X, \mathbb{R})$ of cohomology classes $\{\omega\}$ of Kähler forms. This is an open convex cone.

(b) The closure $\overline{\mathscr{K}}$ of the Kähler cone consists of classes $\{\alpha\} \in H^{1,1}(X, \mathbb{R})$ such that for every $\varepsilon>0$ the sum $\{\alpha+\varepsilon \omega\}$ is Kähler, or equivalently, for every $\varepsilon>0$, there exists a smooth function $\varphi_{\varepsilon}$ on $X$ such that $\alpha+i \partial \bar{\partial} \varphi_{\varepsilon} \geqslant-\varepsilon \omega$. We say that $\overline{\mathscr{K}}$ is the cone of nef $(1,1)$-classes.

(c) The pseudo-effective cone is the set $\mathscr{E} \subset H^{1,1}(X, \mathbb{R})$ of cohomology classes $\{T\}$ of closed positive currents of type $(1,1)$. This is a closed convex cone.

(d) The interior $\mathscr{E}^{\circ}$ of $\mathscr{E}$ consists of classes which still contain a closed positive current after one subtracts $\varepsilon\{\omega\}$ for $\varepsilon>0$ small, in other words, they are classes of closed $(1,1)$-currents $T$ such that $T \geqslant \varepsilon \omega$. Such a current will be called a Kähler current, and we say that $\{T\} \in H^{1,1}(X, \mathbb{R})$ is a big $(1,1)$-class.

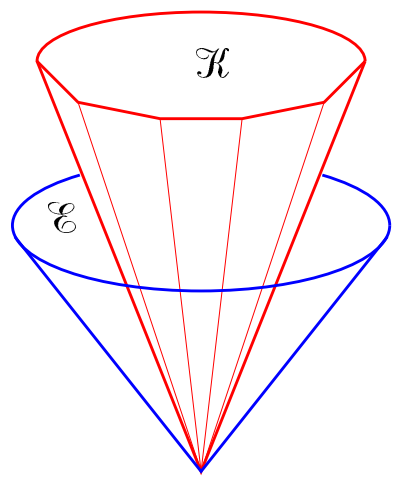

$\mathscr{K}=$ Kähler cone in $H^{1,1}(X, \mathbb{R})$ [open]

$\overline{\mathscr{K}}=$ nef cone in $H^{1,1}(X, \mathbb{R})$ [closure of $\mathscr{K}$ ]

$\mathscr{E}=$ pseudo-effective cone in $H^{1,1}(X, \mathbb{R})$ [closed]

$\mathscr{E}^{\circ}=$ big cone in $H^{1,1}(X, \mathbb{R})$ [interior of $\mathscr{E}$ ]

The openness of $\mathscr{K}$ is clear by definition, and the closedness of $\mathscr{E}$ is a consequence of the fact that bounded sets of currents are weakly compact (as follows from the similar weak compactness property for bounded sets of positive measures). It is then clear that $\overline{\mathscr{K}} \subset \mathscr{E}$.

In spite of the fact that cohomology groups can be defined either in terms of forms or currents, it turns out that the cones $\overline{\mathscr{K}}$ and $\mathscr{E}$ are in general different. To see this, it is enough to observe that a Kähler class $\{\alpha\}$ satisfies $\int_{Y} \alpha^{p}>0$ for every $p$-dimensional analytic set. On the other hand, if $X$ is the surface obtained by blowing-up $\mathbb{P}^{2}$ in one point, then the exceptional divisor $E \simeq \mathbb{P}^{1}$ has a cohomology class $\{\alpha\}$ such that $\int_{E} \alpha=E^{2}=-1$, hence $\{\alpha\} \notin \overline{\mathscr{K}}$, although $\{\alpha\}=\{[E]\} \in \mathscr{E}$.

In case $X$ is projective, all Chern classes $c_{1}(L)$ of line bundles lie by definition in $\mathrm{NS}(X)$, and likewise, all classes of real divisors $D=\sum c_{j} D_{j}, c_{j} \in \mathbb{R}$, lie in $\operatorname{NS}_{\mathbb{R}}(X)$. In 
order to deal with such algebraic classes, we therefore introduce the intersections

$$
\mathscr{K}_{\mathrm{NS}}=\mathscr{K} \cap \mathrm{NS}_{\mathbb{R}}(X), \quad \mathscr{E}_{\mathrm{NS}}=\mathscr{E} \cap \mathrm{NS}_{\mathbb{R}}(X),
$$

and refer to classes of $H^{1,1}(X, \mathbb{R})$ not contained in $\mathrm{NS}_{\mathbb{R}}(X)$ as transcendental classes.

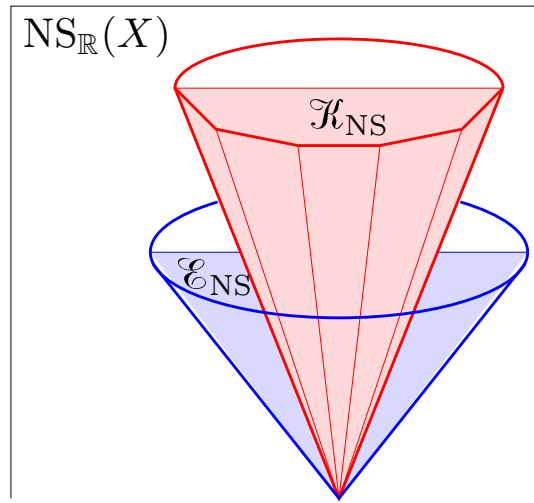

A very important fact is that all four cones $\mathscr{K}_{\mathrm{NS}}, \mathscr{E}_{\mathrm{NS}}, \overline{\mathscr{K}}_{\mathrm{NS}}, \mathscr{E}_{\mathrm{NS}}^{\circ}$ have simple algebraic interpretations.

(3.4) Theorem. Let $X$ be a projective manifold. Then

(a) $\mathscr{K}_{\mathrm{NS}}$ is equal to the open cone $\operatorname{Amp}(X)$ generated by classes of ample (or very ample) divisors $A$ (recall that a divisor $A$ is said to be very ample if the linear system $H^{0}(X, \odot(A))$ provides an embedding of $X$ in projective space).

(b) The interior $\mathscr{E}_{\mathrm{NS}}^{\circ}$ is the cone $\operatorname{Big}(X)$ generated by classes of big divisors, namely divisors $D$ such that $h^{0}(X, \mathscr{O}(k D)) \geqslant c k^{\operatorname{dim} X}$ for $k$ large.

(c) $\mathscr{E}_{\mathrm{NS}}$ is the closure $\overline{\mathrm{Eff}(X)}$ of the cone generated by classes of effective divisors, i.e. divisors $D=\sum c_{j} D_{j}, c_{j} \in \mathbb{R}_{+}$.

(d) The closed cone $\overline{\mathscr{K}}_{\mathrm{NS}}$ consists of the closure $\overline{\operatorname{Nef}(X)}$ of the cone generated by nef divisors $D$ (or nef line bundles $L$ ), namely effective integral divisors $D$ such that $D \cdot C \geqslant 0$ for every curve $C$, also equal to $\overline{\operatorname{Amp}(X)}$.

In other words, the terminology "nef", "big", "pseudo-effective" used for classes of the full transcendental cones appear to be a natural extrapolation of the algebraic case.

Proof. First notice that since all of our cones $\mathscr{C}$ have non empty interior in $\mathrm{NS}_{\mathbb{R}}(X)$ (which is a rational vector space in terms of a basis of elements in $\left.H^{2}(X, \mathbb{Q})\right)$, the rational points $\mathscr{C}_{\mathbb{Q}}:=\mathscr{C} \cap \mathrm{NS}_{\mathbb{Q}}(X), \mathrm{NS}_{\mathbb{Q}}(X)=\mathrm{NS}(X) \otimes_{\mathbb{Z}} \mathbb{Q}$, are dense in each of them.

(a) is therefore just Kodaira's embedding theorem when we look at rational points, and properties (b) and (d) are obtained easily by passing to the closure of the open cones. We will now give details of the proof only for (b) which is possibly slightly more involved.

By looking at points of $\mathscr{C}_{\mathbb{Q}}^{\circ}=\mathscr{E}^{\circ} \cap \mathrm{NS}_{\mathbb{Q}}(X)$ and multiplying by a denominator, it is enough to check that a line bundle $L$ such that $c_{1}(L) \in \mathscr{E}^{\circ}$ is big. However, this means that $L$ possesses a singular Hermitian metric $h_{L}$ such that $\Theta_{L, h_{L}} \geqslant \varepsilon \omega$ for some 
Kähler metric $\omega$. For some integer $p_{0}>0$, we can then produce a singular Hermitian metric with positive curvature and with a given logarithmic pole $h_{L}^{p_{0}} e^{-\theta(z) \log \left|z-x_{0}\right|^{2}}$ in a neighborhood of every point $x_{0} \in X$ (here $\theta$ is a smooth cut-off function supported on a neighborhood of $x_{0}$ ). Then Hörmander's $L^{2}$ existence theorem [Hör65, AV65] can be used to produce sections of $L^{k}$ which generate all jets of order $\left(k / p_{0}\right)-n$ at points $x_{0}$, so that $L$ is big.

Conversely, if $L$ is big and $A$ is a (smooth) very ample divisor, the exact sequence $0 \rightarrow \mathscr{O}_{X}(k L-A) \rightarrow \mathscr{O}_{X}(k L) \rightarrow \mathscr{\sigma}_{A}\left(k L_{\uparrow A}\right) \rightarrow 0$ and the estimates $h^{0}\left(X, \mathscr{\sigma}_{X}(k L)\right) \geqslant c k^{n}$, $h^{0}\left(A, \mathscr{O}_{A}\left(k L_{\uparrow A}\right)\right)=O\left(k^{n-1}\right)$ imply that $\mathscr{O}_{X}(k L-A)$ has a section for $k$ large, thus $k L-A \equiv E$ for some effective divisor $E$. This means that there exists a singular metric $h_{L}$ on $L$ such that

$$
\Theta_{L, h_{L}}=\frac{1}{k}\left(\Theta_{A, h_{A}}+[E]\right) \geqslant \frac{1}{k} \omega
$$

where $\omega=\Theta_{A, h_{A}}$, hence $c_{1}(L) \in \mathscr{E}^{\circ}$.

(3.5) Corollary. If $L$ is nef, then $L$ is big (i.e. $\kappa(L)=n$ ) if and only if $L^{n}>0$. Moreover, if $L$ is nef and big, then for every $\delta>0, L$ has a singular metric $h=e^{-\varphi}$ such that $\max _{x \in X} \nu(\varphi, x) \leqslant \delta$ and $i \Theta_{L, h} \geqslant \varepsilon \omega$ for some $\varepsilon>0$. The metric $h$ can be chosen to be smooth on the complement of a fixed divisor E, with logarithmic poles along $E$.

Proof. By (I 2.10) and the Riemann-Roch formula, we have

$$
h^{0}(X, k L)=\chi(X, k L)+o\left(k^{n}\right)=k^{n} L^{n} / n !+o\left(k^{n}\right),
$$

whence the first statement. By the proof of Theorem 3.4 (b), there exists a singular metric $h_{1}$ on $L$ such that

$$
\frac{i}{2 \pi} \Theta_{L, h_{1}}=\frac{1}{k}\left(\frac{i}{2 \pi} \Theta_{A, h_{A}}+[E]\right) \geqslant \frac{1}{k} \omega, \quad \omega=\frac{i}{2 \pi} \Theta_{A, h_{A}} .
$$

Now, for every $\varepsilon>0$, there is a smooth metric $h_{\varepsilon}$ on $L$ such that $\frac{i}{2 \pi} \Theta_{L, h_{\varepsilon}} \geqslant-\varepsilon \omega$. The convex combination of metrics $h_{\varepsilon}^{\prime}=h_{1}^{k \varepsilon} h_{\varepsilon}^{1-k \varepsilon}$ is a singular metric with poles along $E$ which satisfies

$$
\frac{i}{2 \pi} \Theta_{L, h_{\varepsilon}^{\prime}} \geqslant \varepsilon(\omega+[E])-(1-k \varepsilon) \varepsilon \omega \geqslant k \varepsilon^{2} \omega
$$

Its Lelong numbers are $\varepsilon \nu(E, x)$ and they can be made smaller than $\delta$ by choosing $\varepsilon>0$ small.

We still need a few elementary facts about the numerical dimension of nef line bundles.

(3.6) Definition. Let $L$ be a nef line bundle on a compact Kähler manifold $X$. One defines the numerical dimension of $L$ to be

$$
\operatorname{nd}(L)=\max \left\{k=0, \ldots, n ; c_{1}(L)^{k} \neq 0 \text { in } H^{2 k}(X, \mathbb{R})\right\} .
$$

Notice that if $L$ is nef, each power $c_{1}(L)^{k}$ can be represented by a closed positive current $\Theta_{k} \in c_{1}(L)^{k}$ obtained as a weak limit of powers of smooth positive forms

$$
\Theta_{k}=\lim _{m \rightarrow+\infty}\left(\alpha+\frac{1}{m} \omega+\partial \bar{\partial} \varphi_{m}\right)^{k}, \quad \alpha \in c_{1}(L) .
$$


Such a weak limit exists since $\int_{X}\left(\alpha+\frac{1}{m} \omega+\partial \bar{\partial} \varphi_{m}\right)^{k} \wedge \omega^{n-k}$ is uniformly bounded as $m \rightarrow+\infty$. Then we see that

$$
\int_{X} c_{1}(L)^{k} \wedge \omega^{n-k}=\int_{X} \Theta_{k} \wedge \omega^{n-k}>0 \Longleftrightarrow \Theta_{k} \neq 0 \Longleftrightarrow c_{1}(L)^{k} \neq 0 .
$$

By Corollary 3.5, we have $\kappa(L)=n$ if and only if $\operatorname{nd}(L)=n$. In general, we merely have an inequality.

(3.7) Proposition. If $L$ is a nef line bundle on a compact Kähler manifold $(X, \omega)$, then $\kappa(L) \leqslant \operatorname{nd}(L)$.

Proof. We consider arbitrary irreducible analytic subsets $Z \subset X$ and prove by induction on $p=\operatorname{dim} Z$ that $\kappa\left(L_{\mid Z}\right) \leqslant \operatorname{nd}\left(L_{\mid Z}\right)$ where $\operatorname{nd}\left(L_{\mid Z}\right)$ is the supremum of all integers $k$ such that $c_{1}\left(L_{\mid Z}\right)^{k} \neq 0$, i.e. $\int_{X}[Z] \wedge c_{1}(L)^{k} \wedge \omega^{p-k}>0$. This will prove our statement when $Z=X, p=n$. The statement is trivial if $p=0$, so we suppose now that $p>0$. We can also assume that $r=\kappa\left(L_{\mid Z}\right)>0$, otherwise there is nothing to prove. This implies that a sufficient large multiple $m_{0} L$ has at least two independent sections $\sigma_{0}, \sigma_{1}$ on $Z$. Consider the linear system $\left|a_{0} \sigma_{0}+a_{1} \sigma_{1}\right|, a=\left[a_{0}: a_{1}\right] \in \mathbb{P}_{\mathbb{C}}^{1}$, and take $Y=Y_{a} \subset Z$ to be an irreducible component of the divisor of $\sigma_{a}:=a_{0} \sigma_{0}+a_{1} \sigma_{1}$ which is not a fixed component when $a$ varies. For $m$ sufficiently divisible, $\Phi_{m L_{\mid Z}}$ has rank $r$ at a generic (smooth) point of $Z$, hence the rank of $\left(\Phi_{m L_{\mid Z}}\right)_{\mid Y}$ is $\geqslant r^{\prime}:=\min (r, p-1)$ if $a \in \mathbb{P}_{\mathbb{C}}^{1}$ is itself generic. A fortiori $\operatorname{rank}\left(\Phi_{m L_{\mid Y}}\right) \geqslant r^{\prime}$ (we may even have sections on $Y$ which do not extend to $Z$ ). By the induction hypothesis we find

$$
\int_{X}[Y] \wedge c_{1}(L)^{r^{\prime}} \wedge \omega^{p-1-r^{\prime}}>0 .
$$

Now, we use the fact that $[Z] \wedge c_{1}\left(m_{0} L\right)-[Y]$ can be represented by an effective cycle (the sum of all components $\neq Y$ in the divisor of our generic section $\sigma_{a}$ ). This implies

$$
\int_{X}[Z] \wedge c_{1}(L)^{r^{\prime}+1} \wedge \omega^{p-1-r^{\prime}} \geqslant \frac{1}{m_{0}} \int_{X}[Y] \wedge c_{1}(L)^{r^{\prime}} \wedge \omega^{p-1-r^{\prime}}>0 .
$$

If $r=p$, we have $r^{\prime}=p-1$, hence $r^{\prime}+1=r$ and we are done. If $r<p$, we have $r^{\prime}=r$ and then we use the obvious inequality $\alpha \leqslant C_{0} \omega$ for some representative $\alpha \in c_{1}(L)$ and some $C_{0}>0$ to conclude that

$$
\int_{X}[Z] \wedge c_{1}(L)^{r} \wedge \omega^{p-r} \geqslant \frac{1}{C_{0}} \int_{X}[Z] \wedge c_{1}(L)^{r+1} \wedge \omega^{p-1-r}>0 .
$$

(3.8) Remark. It may happen that $\kappa(L)<\operatorname{nd}(L)$ : take e.g.

$$
L \rightarrow X=X_{1} \times X_{2}
$$

equal to the total tensor product of an ample line bundle $L_{1}$ on a projective manifold $X_{1}$ and of a unitary flat line bundle $L_{2}$ on an elliptic curve $X_{2}$ given by a representation $\pi_{1}\left(X_{2}\right) \rightarrow U(1)$ such that no multiple $k L_{2}$ with $k \neq 0$ is trivial. Then $H^{0}(X, k L)=$ $H^{0}\left(X_{1}, k L_{1}\right) \otimes H^{0}\left(X_{2}, k L_{2}\right)=0$ for $k>0$, and thus $\kappa(L)=-\infty$. However $c_{1}(L)=$ $\operatorname{pr}_{1}^{*} c_{1}\left(L_{1}\right)$ has numerical dimension equal to $\operatorname{dim} X_{1}$. The same example shows that the Kodaira dimension may increase by restriction to a subvariety (if $Y=X_{1} \times\{$ point , then $\left.\kappa\left(L_{\uparrow Y}\right)=\operatorname{dim} Y\right)$. 


\section{Approximation of plurisubharmonic functions via Bergman kernels}

We prove here, as an application of the Ohsawa-Takegoshi $L^{2}$ extension theorem [OT87], that every psh function on a pseudoconvex open set $\Omega \subset \mathbb{C}^{n}$ can be approximated very accurately by functions of the form $c \log |f|$, where $c>0$ and $f$ is a holomorphic function. The main idea is taken from [Dem92]. For other applications to algebraic geometry, see [Dem93] and Demailly-Kollár [DK01]. We first recall the statement of the generalized $L^{2}$ extension theorem; its proof relies on a subtle enhancement of the Bochner-Kodaira technique, and we refer to the litterature for details.

(4.1) Theorem (Ohsawa-Takegoshi [OT87], Manivel [Man93]). Let X be a complex n-dimensional manifold possessing a smooth plurisubharmonic exhaustion function ("weakly pseudoconvex" or "weakly 1-convex" manifold), and a Kähler metric $\omega$. Let $L$ (resp. E) be a Hermitian holomorphic line bundle (resp. a Hermitian holomorphic vector bundle of rank $r$ over $X$ ), and s a global holomorphic section of $E$. Assume that $s$ is generically transverse to the zero section, and let

$$
Y=\left\{x \in X ; s(x)=0, \Lambda^{r} d s(x) \neq 0\right\}, \quad p=\operatorname{dim} Y=n-r
$$

Finally, let $\varphi$ be an arbitrary plurisubharmonic function on $X$. Assume that the $(1,1)$ form $\Theta_{L}+r \frac{i}{2 \pi} \partial \bar{\partial}\left(\log |s|^{2}+\varphi\right)$ is semi-positive and that there is a continuous function $\alpha \geqslant 1$ such that the following two inequalities hold everywhere on $X$ :

(a) $\Theta_{L}+r \frac{i}{2 \pi} \partial \bar{\partial}\left(\log |s|^{2}+\varphi\right) \geqslant \alpha^{-1} \frac{\left\{\Theta_{E} s, s\right\}}{|s|^{2}}$,

(b) $|s| \leqslant e^{-\alpha}$.

Then for every holomorphic section $f_{Y}$ of the line bundle $\Lambda^{n} T_{X}^{*} \otimes L$ over $Y$ such that $\int_{Y}\left|f_{Y}\right|^{2} e^{-\varphi}\left|\Lambda^{r}(d s)\right|^{-2} d V_{\omega}<+\infty$, there exists a holomorphic extension $f_{X}$ of $f_{Y}$ over $X$ such that

$$
\int_{X} \frac{\left|f_{X}\right|^{2} e^{-\varphi}}{|s|^{2 r}(-\log |s|)^{2}} d V_{X, \omega} \leqslant C_{r} \int_{Y} \frac{\left|f_{Y}\right|^{2} e^{-\varphi}}{\left|\Lambda^{r}(d s)\right|^{2}} d V_{Y, \omega}
$$

where $C_{r}$ is a numerical constant depending only on $r$.

(4.2) Theorem. Let $\varphi$ be a plurisubharmonic function on a bounded pseudoconvex open set $\Omega \subset \mathbb{C}^{n}$. For every $m>0$, let $\mathscr{H}_{\Omega}(m \varphi)$ be the Hilbert space of holomorphic functions $f$ on $\Omega$ such that $\int_{\Omega}|f|^{2} e^{-2 m \varphi} d \lambda<+\infty$ and let $\varphi_{m}=\frac{1}{2 m} \log \sum\left|\sigma_{\ell}\right|^{2}$ where $\left(\sigma_{\ell}\right)$ is an orthonormal basis of $\mathscr{H}_{\Omega}(m \varphi)$. Then there are constants $C_{1}, C_{2}>0$ independent of $m$ such that

(a) $\varphi(z)-\frac{C_{1}}{m} \leqslant \varphi_{m}(z) \leqslant \sup _{|\zeta-z|<r} \varphi(\zeta)+\frac{1}{m} \log \frac{C_{2}}{r^{n}}$ for every $z \in \Omega$ and $r<d(z, \partial \Omega)$. In particular, $\varphi_{m}$ converges to $\varphi$ pointwise and in $L_{\text {loc }}^{1}$ topology on $\Omega$ when $m \rightarrow+\infty$ and

(b) $\nu(\varphi, z)-\frac{n}{m} \leqslant \nu\left(\varphi_{m}, z\right) \leqslant \nu(\varphi, z)$ for every $z \in \Omega$.

Proof. (a) Note that $\sum\left|\sigma_{\ell}(z)\right|^{2}$ is the square of the norm of the evaluation linear form $\mathrm{ev}_{z}: f \mapsto f(z)$ on $\mathscr{H}_{\Omega}(m \varphi)$, since $\sigma_{\ell}(z)=\mathrm{ev}_{z}\left(\sigma_{\ell}\right)$ is the $\ell$-th coordinate of $\mathrm{ev}_{z}$ in the 
orthonormal basis $\left(\sigma_{\ell}\right)$. In other words, we have

$$
\sum\left|\sigma_{\ell}(z)\right|^{2}=\sup _{f \in B(1)}|f(z)|^{2}
$$

where $B(1)$ is the unit ball of $\mathscr{H}_{\Omega}(m \varphi)$ (The sum is called the Bergman kernel associated with $\left.\mathscr{H}_{\Omega}(m \varphi)\right)$. As $\varphi$ is locally bounded from above, the $L^{2}$ topology is actually stronger than the topology of uniform convergence on compact subsets of $\Omega$. It follows that the series $\sum\left|\sigma_{\ell}\right|^{2}$ converges uniformly on $\Omega$ and that its sum is real analytic. Moreover, by what we just explained, we have

$$
\varphi_{m}(z)=\sup _{f \in B(1)} \frac{1}{m} \log |f(z)| .
$$

For $z_{0} \in \Omega$ and $r<d\left(z_{0}, \partial \Omega\right)$, the mean value inequality applied to the psh function $|f|^{2}$ implies

$$
\begin{aligned}
\left|f\left(z_{0}\right)\right|^{2} & \leqslant \frac{1}{\pi^{n} r^{2 n} / n !} \int_{|z-z-0|<r}|f(z)|^{2} d \lambda(z) \\
& \leqslant \frac{1}{\pi^{n} r^{2 n} / n !} \exp \left(2 m \sup _{\left|z-z_{0}\right|<r} \varphi(z)\right) \int_{\Omega}|f|^{2} e^{-2 m \varphi} d \lambda .
\end{aligned}
$$

If we take the supremum over all $f \in B(1)$ we get

$$
\varphi_{m}\left(z_{0}\right) \leqslant \sup _{\left|z-z_{0}\right|<r} \varphi(z)+\frac{1}{2 m} \log \frac{1}{\pi^{n} r^{2 n} / n !}
$$

and the second inequality in (a) is proved - as we see, this is an easy consequence of the mean value inequality. Conversely, the Ohsawa-Takegoshi $L^{2}$ extension theorem 4.1 applied to the 0-dimensional subvariety $\left\{z_{0}\right\} \subset \Omega$ and to the trivial bundles $L=\Omega \times \mathbb{C}$ and $E=\Omega \times \mathbb{C}^{n}$, with the section $s(z)=z-z_{0}$ of $E$, shows that for any $a \in \mathbb{C}$ there is a holomorphic function $f$ on $\Omega$ such that $f\left(z_{0}\right)=a$ and

$$
\int_{\Omega}|f|^{2} e^{-2 m \varphi} d \lambda \leqslant C_{3}|a|^{2} e^{-2 m \varphi\left(z_{0}\right)}
$$

where $C_{3}$ only depends on $n$ and $\operatorname{diam} \Omega$. We fix $a$ such that the right hand side is 1 . Then $\|f\| \leqslant 1$ and so we get

$$
\varphi_{m}\left(z_{0}\right) \geqslant \frac{1}{m} \log \left|f\left(z_{0}\right)\right|=\frac{1}{m} \log |a|=\varphi(z)-\frac{\log C_{3}}{2 m} .
$$

The inequalities given in (a) are thus proved. Taking $r=1 / m$, we find that

$$
\lim _{m \rightarrow+\infty} \sup _{|\zeta-z|<1 / m} \varphi(\zeta)=\varphi(z)
$$

by the upper semicontinuity of $\varphi$, and thus $\lim \varphi_{m}(z)=\varphi(z)$, since $\lim \frac{1}{m} \log \left(C_{2} m^{n}\right)=0$.

(b) The above estimates imply

$$
\sup _{\left|z-z_{0}\right|<r} \varphi(z)-\frac{C_{1}}{m} \leqslant \sup _{\left|z-z_{0}\right|<r} \varphi_{m}(z) \leqslant \sup _{\left|z-z_{0}\right|<2 r} \varphi(z)+\frac{1}{m} \log \frac{C_{2}}{r^{n}} .
$$


After dividing by $\log r<0$ when $r \rightarrow 0$, we infer

$$
\frac{\sup _{\left|z-z_{0}\right|<2 r} \varphi(z)+\frac{1}{m} \log \frac{C_{2}}{r^{n}}}{\log r} \leqslant \frac{\sup _{\left|z-z_{0}\right|<r} \varphi_{m}(z)}{\log r} \leqslant \frac{\sup _{\left|z-z_{0}\right|<r} \varphi(z)-\frac{C_{1}}{m}}{\log r},
$$

and from this and definition (1.6), it follows immediately that

$$
\nu(\varphi, x)-\frac{n}{m} \leqslant \nu\left(\varphi_{m}, z\right) \leqslant \nu(\varphi, z) .
$$

Theorem 4.2 implies in a straightforward manner the deep result of [Siu74] on the analyticity of the Lelong number upperlevel sets.

(4.3) Corollary ([Siu74]). Let $\varphi$ be a plurisubharmonic function on a complex manifold $X$. Then, for every $c>0$, the Lelong number upperlevel set

$$
E_{c}(\varphi)=\{z \in X ; \nu(\varphi, z) \geqslant c\}
$$

is an analytic subset of $X$.

Proof. Since analyticity is a local property, it is enough to consider the case of a psh function $\varphi$ on a pseudoconvex open set $\Omega \subset \mathbb{C}^{n}$. The inequalities obtained in Theorem 4.2 (b) imply that

$$
E_{c}(\varphi)=\bigcap_{m \geqslant m_{0}} E_{c-n / m}\left(\varphi_{m}\right)
$$

Now, it is clear that $E_{c}\left(\varphi_{m}\right)$ is the analytic set defined by the equations $\sigma_{\ell}^{(\alpha)}(z)=0$ for all multi-indices $\alpha$ such that $|\alpha|<m c$. Thus $E_{c}(\varphi)$ is analytic as a (countable) intersection of analytic sets.

(4.4) Remark. It can be easily shown that the Lelong numbers of any closed positive $(p, p)$-current coincide (at least locally) with the Lelong numbers of a suitable plurisubharmonic potential $\varphi$ (see [Sko72a]). Hence Siu's theorem also holds true for the Lelong number upperlevel sets $E_{c}(T)$ of any closed positive $(p, p)$-current $T$.

Theorem 4.2 motivates the following definition.

(4.5) Definition. A plurisubharmonic function $\varphi$ on a complex manifold $X$ is said to have analytic singularities if it can be written locally near every point $x_{0} \in X$ as

$$
\varphi(z)=c \log \sum_{1 \leqslant j \leqslant N}\left|g_{j}(z)\right|^{2}+O(1), \quad \text { i.e. up to equivalence of singularities, }
$$

with a family of holomorphic functions $\left(g_{j}\right)$ defined near $x_{0}$ and $c>0$. Also, a closed positive $(1,1)$ current $T$ is said to have analytic singularities if its plurisubharmonic potential has analytic singularities. We also refer to this situation by saying that $\varphi$ or $T$ have logarithmic poles. When $X$ is algebraic, we say that the singularities are algebraic if $c \in \mathbb{Q}_{+}$and the $\left(g_{j}\right)$ are sections of some algebraic line bundle $\mathscr{O}(D), x_{0} \notin \operatorname{Supp} D$.

Notice that by Noetherianity, a convergent series $\log \sum_{j \in \mathbb{N}}\left|g_{j}\right|^{2}$ can be replaced by a finite sum up to equivalence of singularities, thus Theorem 4.2 always produces plurisubharmonic functions $\varphi_{m}$ with analytic singularities. 


\section{Global approximation of closed $(1,1)$-currents on a compact complex manifold}

We take here $X$ to be an arbitrary compact complex manifold (no Kähler assumption is needed). Now, let $T$ be a closed $(1,1)$-current on $X$. We assume that $T$ is quasi-positive, i.e. that there exists a $(1,1)$-form $\gamma$ with continuous coefficients such that $T \geqslant \gamma$; the case of positive currents $(\gamma=0)$ is of course the most important.

(5.1) Lemma. There exists a smooth closed (1,1)-form $\alpha$ representing the same $\partial \bar{\partial}$ cohomology class as $T$ and a quasi-psh function $\varphi$ on $X$ such that $T=\alpha+\frac{i}{\pi} \partial \bar{\partial} \varphi$. (We say that a function $\varphi$ is quasi-psh if its complex Hessian is bounded below by a $(1,1)$-form

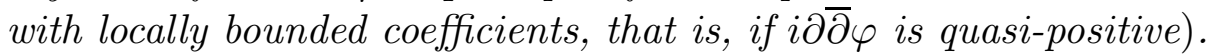

Proof. Select an open covering $\left(U_{j}\right)$ of $X$ by coordinate balls such that $T=\frac{i}{\pi} \partial \bar{\partial} \varphi_{j}$ over $U_{j}$, and construct a global function $\varphi=\sum \theta_{j} \varphi_{j}$ by means of a partition of unity $\left\{\theta_{j}\right\}$ subordinate to $U_{j}$. Now, we observe that $\varphi-\varphi_{k}$ is smooth on $U_{k}$ because all differences $\varphi_{j}-\varphi_{k}$ are smooth in the intersections $U_{j} \cap U_{k}$, and we have the equality $\varphi-\varphi_{k}=\sum \theta_{j}\left(\varphi_{j}-\varphi_{k}\right)$. Therefore $\alpha:=T-\frac{i}{\pi} \partial \bar{\partial} \varphi$ is smooth.

By replacing $T$ with $T-\alpha$ and $\gamma$ with $\gamma-\alpha$, we can assume without loss of generality that $\{T\}=0$, i.e. that $T=\frac{i}{\pi} \partial \bar{\partial} \varphi$ with a quasi-psh function $\varphi$ on $X$ such that $\frac{i}{\pi} \partial \bar{\partial} \varphi \geqslant \gamma$.

Our goal is to approximate $T$ in the weak topology by currents $T_{m}=\frac{i}{\pi} \partial \bar{\partial} \varphi_{m}$ such their potentials $\varphi_{m}$ have analytic singularities in the sense of Definition 4.5 , more precisely, defined on a neighborhood $V_{x_{0}}$ of any point $x_{0} \in X$ in the form $\varphi_{m}(z)=$ $c_{m} \log \sum_{j}\left|\sigma_{j, m}\right|^{2}+O(1)$, where $c_{m}>0$ and the $\sigma_{j, m}$ are holomorphic functions on $V_{x_{0}}$.

We select a finite covering $\left(W_{\nu}\right)$ of $X$ with open coordinate charts, and shrink them a little to be on the safe side. Given $\delta>0$, we take in each $W_{\nu}$ a maximal family of points with (coordinate) distance to the boundary $\geqslant 3 \delta$ and mutual distance $\geqslant \delta / 2$. In this way, we get for $\delta>0$ small a finite covering of $X$ by open balls $U_{j}^{\prime}$ of radius $\delta$ (actually every point is even at distance $\leqslant \delta / 2$ of one of the centers, otherwise the family of points would not be maximal), such that the concentric ball $U_{j}$ of radius $2 \delta$ is relatively compact in the corresponding chart $W_{\nu}$. Let $\tau_{j}: U_{j} \longrightarrow B\left(a_{j}, 2 \delta\right)$ be the isomorphism given by the coordinates of $W_{\nu}$; by taking $\delta>0$ small enough, we can assume that the coordinates of $U_{j}$ extend to $U_{j} \cup U_{k}$ whenever $U_{j} \cap U_{k} \neq \emptyset$. Let $\varepsilon(\delta)$ be a modulus of continuity for $\gamma$ on the sets $U_{j}$, such that $\lim _{\delta \rightarrow 0} \varepsilon(\delta)=0$ and $\gamma_{x}-\gamma_{x^{\prime}} \leqslant \frac{1}{2} \varepsilon(\delta) \omega_{x}$ for all $x, x^{\prime} \in U_{j}$. We denote by $\gamma_{j}$ the $(1,1)$-form with constant coefficients on $B\left(a_{j}, 2 \delta\right)$ such that $\tau_{j}^{*} \gamma_{j}$ coincides with $\gamma-\varepsilon(\delta) \omega$ at $\tau_{j}^{-1}\left(a_{j}\right)$. Then we have

$$
0 \leqslant \gamma-\tau_{j}^{*} \gamma_{j} \leqslant 2 \varepsilon(\delta) \omega \quad \text { on } U_{j}
$$

for $\delta>0$ small. We set $\varphi_{j}=\varphi \circ \tau_{j}^{-1}$ on $B\left(a_{j}, 2 \delta\right)$ and let $q_{j}$ be the homogeneous quadratic function in $z-a_{j}$ such that $\frac{i}{\pi} \partial \bar{\partial} q_{j}=\gamma_{j}$ on $B\left(a_{j}, 2 \delta\right)$. Then $\varphi_{j}-q_{j}$ is plurisubharmonic on $B\left(a_{j}, 2 \delta\right)$ since

$$
\frac{i}{\pi} \partial \bar{\partial}\left(\left(\varphi_{j}-q_{j}\right) \circ \tau_{j}\right)=T-\tau_{j}^{*} \gamma_{j} \geqslant \gamma-\tau_{j}^{*} \gamma_{j} \geqslant 0
$$

We let $U_{j}^{\prime} \subset \subset U_{j}^{\prime \prime} \subset \subset U_{j}$ be the concentric balls of radii $\delta, 1.5 \delta, 2 \delta$ respectively. On each open set $U_{j}$ the function $\psi_{j}:=\varphi-q_{j} \circ \tau_{j}=\left(\varphi_{j}-q_{j}\right) \circ \tau_{j}$ is plurisubharmonic, so 
Theorem 4.2 applied with $\Omega=U_{j} \simeq B\left(a_{j}, 2 \delta\right)$ produces functions

$$
\psi_{j, m}=\frac{1}{2 m} \log \sum_{\ell}\left|\sigma_{j, \ell}\right|^{2}, \quad\left(\sigma_{j, \ell}\right)=\text { basis of } \mathscr{H}_{U_{j}}\left(m \psi_{j}\right) .
$$

The functions $\psi_{j, m}+q_{j} \circ \tau_{j}$ on $U_{j}$ then have to be glued together by a partition of unity technique. For this, we rely on the following "discrepancy" lemma, estimating the variation of the approximating functions on overlapping balls.

(5.5) Lemma. There is a constant $C$ independent of $m$ and $\delta$ such that the quasi-psh functions $w_{j, m}=2 m\left(\psi_{j, m}+q_{j} \circ \tau_{j}\right)$, i.e.

$$
w_{j, m}(x)=2 m q_{j} \circ \tau_{j}(x)+\log \sum_{\ell}\left|\sigma_{j, \ell}(x)\right|^{2}, \quad x \in U_{j}^{\prime \prime},
$$

satisfy

$$
\left|w_{j, m}-w_{k, m}\right| \leqslant C\left(\log \delta^{-1}+m \varepsilon(\delta) \delta^{2}\right) \quad \text { on } \quad U_{j}^{\prime \prime} \cap U_{k}^{\prime \prime}
$$

Proof. The details will be left as an exercise to the reader. The main idea is the following: for any holomorphic function $f_{j} \in \mathscr{H}_{U_{j}}\left(m \psi_{j}\right)$, a $\bar{\partial}$ equation $\bar{\partial} u=\bar{\partial}\left(\theta f_{j}\right)$ can be solved on $U_{k}$, where $\theta$ is a cut-off function with support in $U_{j}^{\prime \prime} \cap U_{k}^{\prime \prime}$, on a ball of radius $<\delta / 4$, equal to 1 on the ball of radius $\delta / 8$ centered at a given point $x_{0} \in U_{j}^{\prime \prime} \cap U_{k}^{\prime \prime}$, with $|\bar{\partial} \theta|=O\left(\delta^{-1}\right)$. We apply the $L^{2}$ estimate with respect to the weight $(n+1) \log \left|x-x_{0}\right|^{2}+2 m \psi_{k}$, where the first term is picked up so as to force the solution $u$ to vanish at $x_{0}$, in such a way that $F_{k}=u-\theta f_{j}$ is holomorphic and $F_{k}\left(x_{0}\right)=f_{j}\left(x_{0}\right)$. The discrepancy between the weights on $U_{j}^{\prime \prime}$ and $U_{k}^{\prime \prime}$ is given by

$$
\psi_{j}-\psi_{k}=-\left(q_{j} \circ \tau_{j}-q_{k} \circ \tau_{k}\right) .
$$

By re-centering the quadratic functions at $\tau_{j}\left(x_{0}\right)$, resp. $\tau_{k}\left(x_{0}\right)$, we can write

$$
q_{j} \circ \tau_{j}-q_{k} \circ \tau_{k}=\operatorname{Re} G_{j k}+R_{j k}
$$

where $G_{j k}$ is holomorphic on $U_{j} \cup U_{k}$ [equal to a difference of linear forms in the coordinates of $B\left(a_{j}, 2 \delta\right)$ and $\left.B\left(a_{k}, 2 \delta\right)\right], G_{j k}\left(x_{0}\right)=q_{j} \circ \tau_{j}\left(x_{0}\right)-q_{k} \circ \tau_{k}\left(x_{0}\right)$ and $R_{j k}=O\left(\varepsilon(\delta) \delta^{2}\right)$ is a remainder term coming from the change of coordinates and the slight discrepancy between $\partial \bar{\partial}\left(q_{j} \circ \tau_{j}\right)$ and $\partial \bar{\partial}\left(q_{k} \circ \tau_{k}\right)$ at the common point $x_{0}$, with $R_{j k}\left(x_{0}\right)=0$. In this way, we get

$$
\left|e^{m G_{j k}}\right|^{2} e^{-m \psi_{k}}=e^{-m \psi_{j}-2 m R_{j k}},
$$

so that we have a uniform control of the $L^{2}$ norm of the solution $f_{k}=e^{m G_{j k}} F_{k}=$ $e^{m G_{j k}}\left(u-\theta f_{j}\right)$ of the form

$$
\int_{U_{k}}\left|f_{k}\right|^{2} e^{-2 m \psi_{k}} \leqslant C \delta^{-2 n-4} e^{m O\left(\varepsilon(\delta) \delta^{2}\right)} \int_{U_{j}}\left|f_{j}\right|^{2} e^{-2 m \psi_{j}} .
$$

The required estimate follows, using the equality

$$
e^{2 m \psi_{j, m}(x)}=\sum_{\ell}\left|\sigma_{j, \ell}(x)\right|^{2}=\sup _{f \in \mathscr{H}_{U_{j}}\left(m \psi_{j}\right),\|f\| \leqslant 1}|f(x)|^{2} \quad \text { on } U_{j},
$$


and the analogous equality on $U_{k}$.

Now, the actual glueing of our quasi-psh functions is performed using the following elementary partition of unity calculation.

(5.6) Lemma. Let $U_{j}^{\prime} \subset \subset U_{j}^{\prime \prime}$ be locally finite open coverings of a complex manifold $X$ by relatively compact open sets, and let $\theta_{j}$ be smooth nonnegative functions with support in $U_{j}^{\prime \prime}$, such that $\theta_{j} \leqslant 1$ on $U_{j}^{\prime \prime}$ and $\theta_{j}=1$ on $U_{j}^{\prime}$. Let $A_{j} \geqslant 0$ be such that

$$
i\left(\theta_{j} \partial \bar{\partial} \theta_{j}-\partial \theta_{j} \wedge \bar{\partial} \theta_{j}\right) \geqslant-A_{j} \omega \quad \text { on } \quad U_{j}^{\prime \prime} \backslash U_{j}^{\prime}
$$

for some positive $(1,1)$-form $\omega$. Finally, let $w_{j}$ be quasi-psh functions on $U_{j}$ with the property that $i \partial \bar{\partial} w_{j} \geqslant \gamma$ for some real $(1,1)$-form $\gamma$ on $M$, and let $C_{j}$ be constants such that

$$
w_{j}(x) \leqslant C_{j}+\sup _{k \neq j, U_{k}^{\prime} \ni x} w_{k}(x) \quad \text { on } \quad U_{j}^{\prime \prime} \backslash U_{j}^{\prime} .
$$

Then the function $w=\log \left(\sum \theta_{j}^{2} e^{w_{j}}\right)$ is quasi-psh and satisfies

$$
i \partial \bar{\partial} w \geqslant \gamma-2\left(\sum_{j} \mathbb{1}_{U_{j}^{\prime \prime} \backslash U_{j}^{\prime}} A_{j} e^{C_{j}}\right) \omega .
$$

Proof. If we set $\alpha_{j}=\theta_{j} \partial w_{j}+2 \partial \theta_{j}$, a straightforward computation shows that

$$
\begin{aligned}
\partial w & =\frac{\sum\left(\theta_{j}^{2} \partial w_{j}+2 \theta_{j} \partial \theta_{j}\right) e^{w_{j}}}{\sum \theta_{j}^{2} e^{w_{j}}}=\frac{\sum \theta_{j} e^{w_{j}} \alpha_{j}}{\sum \theta_{j}^{2} e^{w_{j}}}, \\
\partial \bar{\partial} w & =\frac{\sum\left(\alpha_{j} \wedge \bar{\alpha}_{j}+\theta_{j}^{2} \partial \bar{\partial} w_{j}+2 \theta_{j} \partial \bar{\partial} \theta_{j}-2 \partial \theta_{j} \wedge \bar{\partial} \theta_{j}\right) e^{w_{j}}}{\sum \theta_{j}^{2} e^{w_{j}}}-\frac{\sum_{j, k} \theta_{j} e^{w_{j}} \theta_{k} e^{w_{k}} \alpha_{j} \wedge \bar{\alpha}_{k}}{\left(\sum \theta_{j}^{2} e^{w_{j}}\right)^{2}} \\
& =\frac{\sum_{j<k}\left|\theta_{j} \alpha_{k}-\theta_{k} \alpha_{j}\right|^{2} e^{w_{j}} e^{w_{k}}}{\left(\sum \theta_{j}^{2} e^{w_{j}}\right)^{2}}+\frac{\sum \theta_{j}^{2} e^{w_{j}} \partial \bar{\partial} w_{j}}{\sum \theta_{j}^{2} e^{w_{j}}}+\frac{\sum\left(2 \theta_{j} \partial \bar{\partial} \theta_{j}-2 \partial \theta_{j} \wedge \bar{\partial} \theta_{j}\right) e^{w_{j}}}{\sum \theta_{j}^{2} e^{w_{j}}}
\end{aligned}
$$

by using the Legendre identity. The first term in the last line is nonnegative and the second one is $\geqslant \gamma$. In the third term, if $x$ is in the support of $\theta_{j} \partial \bar{\partial} \theta_{j}-\partial \theta_{j} \wedge \bar{\partial} \theta_{j}$, then $x \in U_{j}^{\prime \prime} \backslash U_{j}^{\prime}$ and so $w_{j}(x) \leqslant C_{j}+w_{k}(x)$ for some $k \neq j$ with $U_{k}^{\prime} \ni x$ and $\theta_{k}(x)=1$. This gives

$$
i \frac{\sum\left(2 \theta_{j} \partial \bar{\partial} \theta_{j}-2 \partial \theta_{j} \wedge \bar{\partial} \theta_{j}\right) e^{w_{j}}}{\sum \theta_{j}^{2} e^{w_{j}}} \geqslant-2 \sum_{j} \mathbb{1}_{U_{j}^{\prime \prime} \backslash U_{j}^{\prime}} e^{C_{j}} A_{j} \omega .
$$

The expected lower bound follows.

We apply Lemma 5.6 to functions $\widetilde{w}_{j, m}$ which are just slight modifications of the functions $w_{j, m}=2 m\left(\psi_{j, m}+q_{j} \circ \tau_{j}\right)$ occurring in Lemma 5.5 :

$$
\begin{aligned}
\widetilde{w}_{j, m}(x) & =w_{j, m}(x)+2 m\left(\frac{C_{1}}{m}+C_{3} \varepsilon(\delta)\left(\delta^{2} / 2-\left|\tau_{j}(x)\right|^{2}\right)\right) \\
& =2 m\left(\psi_{j, m}(x)+q_{j} \circ \tau_{j}(x)+\frac{C_{1}}{m}+C_{3} \varepsilon(\delta)\left(\delta^{2} / 2-\left|\tau_{j}(x)\right|^{2}\right)\right)
\end{aligned}
$$


where $x \mapsto z=\tau_{j}(x)$ is a local coordinate identifying $U_{j}$ to $B(0,2 \delta), C_{1}$ is the constant occurring in Lemma 5.5 and $C_{3}$ is a sufficiently large constant. It is easy to see that we can take $A_{j}=C_{4} \delta^{-2}$ in Lemma 5.6. We have

$$
\widetilde{w}_{j, m} \geqslant w_{j, m}+2 C_{1}+m \frac{C_{3}}{2} \varepsilon(\delta) \delta^{2} \quad \text { on } B\left(x_{j}, \delta / 2\right) \subset U_{j}^{\prime},
$$

since $\left|\tau_{j}(x)\right| \leqslant \delta / 2$ on $B\left(x_{j}, \delta / 2\right)$, while

$$
\widetilde{w}_{j, m} \leqslant w_{j, m}+2 C_{1}-m C_{3} \varepsilon(\delta) \delta^{2} \quad \text { on } U_{j}^{\prime \prime} \backslash U_{j}^{\prime} .
$$

For $m \geqslant m_{0}(\delta)=\left(\log \delta^{-1} /\left(\varepsilon(\delta) \delta^{2}\right)\right.$, Lemma 5.5 implies $\left|w_{j, m}-w_{k, m}\right| \leqslant C_{5} m \varepsilon(\delta) \delta^{2}$ on $U_{j}^{\prime \prime} \cap U_{k}^{\prime \prime}$. Hence, for $C_{3}$ large enough, we get

$$
\widetilde{w}_{j, m}(x) \leqslant \sup _{k \neq j, B\left(x_{k}, \delta / 2\right) \ni x} w_{k, m}(x) \leqslant \sup _{k \neq j, U_{k}^{\prime} \ni x} w_{k, m}(x) \quad \text { on } \quad U_{j}^{\prime \prime} \backslash U_{j}^{\prime}
$$

and we can take $C_{j}=0$ in the hypotheses of Lemma 5.6. The associated function $w=\log \left(\sum \theta_{j}^{2} e^{\widetilde{w}_{j, m}}\right)$ is given by

$$
w=\log \sum_{j} \theta_{j}^{2} \exp \left(2 m\left(\psi_{j, m}+q_{j} \circ \tau_{j}+\frac{C_{1}}{m}+C_{3} \varepsilon(\delta)\left(\delta^{2} / 2-\left|\tau_{j}\right|^{2}\right)\right)\right) .
$$

If we define $\varphi_{m}=\frac{1}{2 m} w$, we get

$$
\varphi_{m}(x):=\frac{1}{2 m} w(x) \geqslant \psi_{j, m}(x)+q_{j} \circ \tau_{j}(x)+\frac{C_{1}}{m}+\frac{C_{3}}{4} \varepsilon(\delta) \delta^{2}>\varphi(x)
$$

in view of Lemma 5.5 , by picking an index $j$ such that $x \in B\left(x_{j}, \delta / 2\right)$. In the opposite direction, the maximum number $N$ of overlapping balls $U_{j}$ does not depend on $\delta$, and we thus get

$$
w \leqslant \log N+2 m\left(\max _{j}\left\{\psi_{j, m}(x)+q_{j} \circ \tau_{j}(x)\right\}+\frac{C_{1}}{m}+\frac{C_{3}}{2} \varepsilon(\delta) \delta^{2}\right) .
$$

By definition of $\psi_{j}$ we have $\sup _{|\zeta-x|<r} \psi_{j}(\zeta) \leqslant \sup _{|\zeta-x|<r} \varphi(\zeta)-q_{j} \circ \tau_{j}(x)+C_{5} r$ thanks to the uniform Lipschitz continuity of $q_{j} \circ \tau_{j}$, thus by Lamme 5.5 again we find

$$
\varphi_{m}(x) \leqslant \frac{\log N}{2 m}+\sup _{|\zeta-x|<r} \varphi(\zeta)+\frac{C_{1}}{m}+\frac{1}{m} \log \frac{C_{2}}{r^{n}}+\frac{C_{3}}{2} \varepsilon(\delta) \delta^{2}+C_{5} r .
$$

By taking for instance $r=1 / m$ and $\delta=\delta_{m} \rightarrow 0$, we see that $\varphi_{m}$ converges to $\varphi$. On the other hand (5.2) implies $\frac{i}{\pi} \partial \bar{\partial} q_{j} \circ \tau_{j}(x)=\tau_{j}^{*} \gamma_{j} \geqslant \gamma-2 \varepsilon(\delta) \omega$, thus

$$
\frac{i}{\pi} \partial \bar{\partial} \widetilde{w}_{j, m} \geqslant 2 m\left(\gamma-C_{6} \varepsilon(\delta) \omega\right)
$$

Lemma 5.6 then produces the lower bound

$$
\frac{i}{\pi} \partial \bar{\partial} w \geqslant 2 m\left(\gamma-C_{6} \varepsilon(\delta) \omega\right)-C_{7} \delta^{-2} \omega
$$


whence

$$
\frac{i}{\pi} \partial \bar{\partial} \varphi_{m} \geqslant \gamma-C_{8} \varepsilon(\delta) \omega
$$

for $m \geqslant m_{0}(\delta)=\left(\log \delta^{-1}\right) /\left(\varepsilon(\delta) \delta^{2}\right)$. We can fix $\delta=\delta_{m}$ to be the smallest value of $\delta>0$ such that $m_{0}(\delta) \leqslant m$, then $\delta_{m} \rightarrow 0$ and we have obtained a sequence of quasi-psh functions $\varphi_{m}$ satisfying the following properties.

(5.7) Theorem. Let $\varphi$ be a quasi-psh function on a compact complex manifold $X$ such that $\frac{i}{\pi} \partial \bar{\partial} \varphi \geqslant \gamma$ for some continuous $(1,1)$-form $\gamma$. Then there is a sequence of quasi-psh functions $\varphi_{m}$ such that $\varphi_{m}$ has the same singularities as a logarithm of a sum of squares of holomorphic functions and a decreasing sequence $\varepsilon_{m}>0$ converging to 0 such that

(a) $\varphi(x)<\varphi_{m}(x) \leqslant \sup _{|\zeta-x|<r} \varphi(\zeta)+C\left(\frac{|\log r|}{m}+r+\varepsilon_{m}\right)$

with respect to coordinate open sets covering $X$. In particular, $\varphi_{m}$ converges to $\varphi$ pointwise and in $L^{1}(X)$ and

(b) $\nu(\varphi, x)-\frac{n}{m} \leqslant \nu\left(\varphi_{m}, x\right) \leqslant \nu(\varphi, x)$ for every $x \in X$;

(c) $\frac{i}{\pi} \partial \bar{\partial} \varphi_{m} \geqslant \gamma-\varepsilon_{m} \omega$.

In particular, we can apply this to an arbitrary positive or quasi-positive closed $(1,1)$-current $T=\alpha+\frac{i}{\pi} \partial \bar{\partial} \varphi$.

(5.8) Corollary. Let $T$ be a quasi-positive closed (1,1)-current on a compact complex manifold $X$ such that $T \geqslant \gamma$ for some continuous $(1,1)$-form $\gamma$. Then there is a sequence of currents $T_{m}$ whose local potentials have the same singularities as $1 / \mathrm{m}$ times a logarithm of a sum of squares of holomorphic functions and a decreasing sequence $\varepsilon_{m}>0$ converging to 0 such that

(a) $T_{m}$ converges weakly to $T$,

(b) $\nu(T, x)-\frac{n}{m} \leqslant \nu\left(T_{m}, x\right) \leqslant \nu(T, x)$ for every $x \in X$;

(c) $T_{m} \geqslant \gamma-\varepsilon_{m} \omega$.

We say that our currents $T_{m}$ are approximations of $T$ with logarithmic poles.

By using blow-ups of $X$, the structure of the currents $T_{m}$ can be better understood. In fact, consider the coherent ideals $\mathscr{F}_{m}$ generated locally by the holomorphic functions $\left(\sigma_{j, m}^{(k)}\right)$ on $U_{k}$ in the local approximations

$$
\varphi_{k, m}=\frac{1}{2 m} \log \sum_{j}\left|\sigma_{j, m}^{(k)}\right|^{2}+O(1)
$$

of the potential $\varphi$ of $T$ on $U_{k}$. These ideals are in fact globally defined, because the local ideals $\mathscr{f}_{m}^{(k)}=\left(\sigma_{j, m}^{(k)}\right)$ are integrally closed, and they coincide on the intersections $U_{k} \cap U_{\ell}$ as they have the same order of vanishing by the proof of Lemma 5.5. By Hironaka [Hir64], we can find a composition of blow-ups with smooth centers $\mu_{m}: \widetilde{X}_{m} \rightarrow X$ such 
that $\mu_{m}^{*} \mathscr{F}_{m}$ is an invertible ideal sheaf associated with a normal crossing divisor $E_{m}$. Now, we can write

$$
\mu_{m}^{*} \varphi_{k, m}=\varphi_{k, m} \circ \mu_{m}=\frac{1}{m} \log \left|s_{E_{m}}\right|+\widetilde{\varphi}_{k, m}
$$

where $s_{E_{m}}$ is the canonical section of $\mathscr{O}\left(-E_{m}\right)$ and $\widetilde{\varphi}_{k, m}$ is a smooth potential. This implies

$$
\mu_{m}^{*} T_{m}=\frac{1}{m}\left[E_{m}\right]+\beta_{m}
$$

where $\left[E_{m}\right]$ is the current of integration over $E_{m}$ and $\beta_{m}$ is a smooth closed (1,1)-form which satisfies the lower bound $\beta_{m} \geqslant \mu_{m}^{*}\left(\gamma-\varepsilon_{m} \omega\right)$. (Recall that the pull-back of a closed $(1,1)$-current by a holomorphic map $f$ is always well-defined, by taking a local plurisubharmonic potential $\varphi$ such that $T=i \partial \bar{\partial} \varphi$ and writing $\left.f^{*} T=i \partial \bar{\partial}(\varphi \circ f)\right)$. In the remainder of this section, we derive from this a rather important geometric consequence, first appeared in [DP04]). We need two related definitions.

(5.10) Definition. A Kähler current on a compact complex space $X$ is a closed positive current $T$ of bidegree $(1,1)$ which satisfies $T \geqslant \varepsilon \omega$ for some $\varepsilon>0$ and some smooth positive Hermitian form $\omega$ on $X$.

(5.11) Definition. A compact complex manifold is said to be in the Fujiki class $\mathscr{C}$ if it is bimeromorphic to a Kähler manifold (or equivalently, using Hironaka's desingularization theorem, if it admits a proper Kähler modification).

(5.12) Theorem. A compact complex manifold $X$ is bimeromorphic to a Kähler manifold (i.e. $X \in \mathscr{C}$ ) if and only if it admits a Kähler current.

Proof. If $X$ is bimeromorphic to a Kähler manifold $Y$, Hironaka's desingularization theorem implies that there exists a blow-up $\widetilde{Y}$ of $Y$ (obtained by a sequence of blow-ups with smooth centers) such that the bimeromorphic map from $Y$ to $X$ can be resolved into a modification $\mu: \widetilde{Y} \rightarrow X$. Then $\widetilde{Y}$ is Kähler and the push-forward $T=\mu_{*} \widetilde{\omega}$ of a Kähler form $\widetilde{\omega}$ on $\widetilde{Y}$ provides a Kähler current on $X$. In fact, if $\omega$ is a smooth Hermitian form on $X$, there is a constant $C$ such that $\mu^{*} \omega \leqslant C \widetilde{\omega}$ (by compactness of $\widetilde{Y}$ ), hence

$$
T=\mu_{*} \widetilde{\omega} \geqslant \mu_{*}\left(C^{-1} \mu^{*} \omega\right)=C^{-1} \omega .
$$

Conversely, assume that $X$ admits a Kähler current $T \geqslant \varepsilon \omega$. By Theorem 5.8 (c), there exists a Kähler current $\widetilde{T}=T_{m} \geqslant \frac{\varepsilon}{2} \omega$ (with $m \gg 1$ so large that $\varepsilon_{m} \leqslant \varepsilon / 2$ ) in the same $\partial \bar{\partial}$-cohomology class as $T$, possessing logarithmic poles. Observation (5.9) implies the existence of a composition of blow-ups $\mu: \widetilde{X} \rightarrow X$ such that

$$
\mu^{*} \widetilde{T}=[\widetilde{E}]+\widetilde{\beta} \quad \text { on } \widetilde{X},
$$

where $\widetilde{E}$ is a $\mathbb{Q}$-divisor with normal crossings and $\widetilde{\beta}$ a smooth closed $(1,1)$-form such that $\widetilde{\beta} \geqslant \frac{\varepsilon}{2} \mu^{*} \omega$. In particular $\widetilde{\beta}$ is positive outside the exceptional locus of $\mu$. This is not enough yet to produce a Kähler form on $\tilde{X}$, but we are not very far. Suppose that $\tilde{X}$ is obtained as a tower of blow-ups

$$
\widetilde{X}=X_{N} \rightarrow X_{N-1} \rightarrow \cdots \rightarrow X_{1} \rightarrow X_{0}=X
$$


where $X_{j+1}$ is the blow-up of $X_{j}$ along a smooth center $Y_{j} \subset X_{j}$. Denote by $S_{j+1} \subset X_{j+1}$ the exceptional divisor, and let $\mu_{j}: X_{j+1} \rightarrow X_{j}$ be the blow-up map. Now, we use the following simple

(5.13) Lemma. For every Kähler current $T_{j}$ on $X_{j}$, there exists $\varepsilon_{j+1}>0$ and a smooth form $u_{j+1}$ in the $\partial \bar{\partial}$-cohomology class of $\left[S_{j+1}\right]$ such that

$$
T_{j+1}=\mu_{j}^{*} T_{j}-\varepsilon_{j+1} u_{j+1}
$$

is a Kähler current on $X_{j+1}$.

Proof. The line bundle $\mathcal{O}\left(-S_{j+1}\right) \mid S_{j+1}$ is equal to $\mathscr{O}_{P\left(N_{j}\right)}(1)$ where $N_{j}$ is the normal bundle to $Y_{j}$ in $X_{j}$. Pick an arbitrary smooth Hermitian metric on $N_{j}$, use this metric to get an induced Fubini-Study metric on $\mathscr{\sigma}_{P\left(N_{j}\right)}(1)$, and finally extend this metric as a smooth Hermitian metric on the line bundle $\mathscr{G}\left(-S_{j+1}\right)$. Such a metric has positive curvature along tangent vectors of $X_{j+1}$ which are tangent to the fibers of $S_{j+1}=$ $P\left(N_{j}\right) \rightarrow Y_{j}$. Assume furthermore that $T_{j} \geqslant \delta_{j} \omega_{j}$ for some Hermitian form $\omega_{j}$ on $X_{j}$ and a suitable $0<\delta_{j} \ll 1$. Then

$$
\mu_{j}^{*} T_{j}-\varepsilon_{j+1} u_{j+1} \geqslant \delta_{j} \mu_{j}^{*} \omega_{j}-\varepsilon_{j+1} u_{j+1}
$$

where $\mu_{j}^{*} \omega_{j}$ is semi-positive on $X_{j+1}$, positive definite on $X_{j+1} \backslash S_{j+1}$, and also positive definite on tangent vectors of $T_{X_{j+1} \mid S_{j+1}}$ which are not tangent to the fibers of $S_{j+1} \rightarrow Y_{j}$. The statement is then easily proved by taking $\varepsilon_{j+1} \ll \delta_{j}$ and by using an elementary compactness argument on the unit sphere bundle of $T_{X_{j+1}}$ associated with any given Hermitian metric.

End of proof of Theorem 5.12. If $\widetilde{u}_{j}$ is the pull-back of $u_{j}$ to the final blow-up $\tilde{X}$, we conclude inductively that $\mu^{*} \widetilde{T}-\sum \varepsilon_{j} \widetilde{u}_{j}$ is a Kähler current. Therefore the smooth form

$$
\widetilde{\omega}:=\widetilde{\beta}-\sum \varepsilon_{j} \widetilde{u}_{j}=\mu^{*} \widetilde{T}-\sum \varepsilon_{j} \widetilde{u}_{j}-[\widetilde{E}]
$$

is Kähler and we see that $\widetilde{X}$ is a Kähler manifold.

(5.14) Remark. A special case of Theorem 5.12 is the following characterization of Moishezon varieties (i.e. manifolds which are bimeromorphic to projective algebraic varieties or, equivalently, whose algebraic dimension is equal to their complex dimension):

A compact complex manifold $X$ is Moishezon if and only if $X$ possesses a Kähler current $T$ such that the De Rham cohomology class $\{T\}$ is rational, i.e. $\{T\} \in H^{2}(X, \mathbb{Q})$.

In fact, in the above proof, we get an integral current $T$ if we take the push forward $T=\mu_{*} \widetilde{\omega}$ of an integral ample class $\{\widetilde{\omega}\}$ on $Y$, where $\mu: Y \rightarrow X$ is a projective model of $Y$. Conversely, if $\{T\}$ is rational, we can take the $\varepsilon_{j}$ 's to be rational in Lemma 5.13. This produces at the end a Kähler metric $\widetilde{\omega}$ with rational De Rham cohomology class on $\widetilde{X}$. Therefore $\widetilde{X}$ is projective by the Kodaira embedding theorem. This result was already observed in [JS93] (see also [Bon93, Bon98] and Section III 6 for a more general perspective based on a singular holomorphic Morse inequalities). 
(5.15) Remark. Hodge decomposition also holds true for manifolds $X \in \mathscr{C}$. In fact let $\mu: \widetilde{X} \rightarrow X$ be a modification such that $\widetilde{X}$ is Kähler. Then there are natural morphisms

$$
\mu^{*}: H_{\frac{p}{\partial}}^{p, q}(X, \mathbb{C}) \rightarrow H_{\frac{p}{\partial}}^{p, q}(\widetilde{X}, \mathbb{C}), \quad \mu_{*}: H_{\bar{\partial}}^{p, q}(\widetilde{X}, \mathbb{C}) \rightarrow H_{\frac{p}{\partial}}^{p, q}(X, \mathbb{C})
$$

induced respectively by the pull-back of smooth forms (resp. the direct image of currents). Clearly, $\mu_{*} \circ \mu^{*}=\mathrm{Id}$, therefore $\mu^{*}$ is injective and $\mu_{*}$ surjective, and similar results hold true for Bott-Chern cohomology or De Rham cohomology. It follows easily from this that the $\partial \bar{\partial}$-lemma still holds true for $X \in \mathscr{C}$, and that there are isomorphisms

$$
H_{\mathrm{BC}}^{p, q}(X, \mathbb{C}) \rightarrow H_{\frac{p}{\partial}}^{p, q}(X, \mathbb{C}), \quad \bigoplus_{p+q=k} H_{\mathrm{BC}}^{p, q}(X, \mathbb{C}) \rightarrow H_{\mathrm{DR}}^{k}(X, \mathbb{C})
$$

\section{Zariski decomposition and mobile intersections}

Let $X$ be compact Kähler and let $\alpha \in \mathscr{E}^{\circ}$ be in the interior of the pseudo-effective cone. In analogy with the algebraic context such a class $\alpha$ is called "big", and it can then be represented by a Kähler current $T$, i.e. a closed positive $(1,1)$-current $T$ such that $T \geqslant \delta \omega$ for some smooth Hermitian metric $\omega$ and a constant $\delta \ll 1$. We first need a variant of the approximation theorem proved in Section 5.

(6.1) Regularization theorem for currents. Let $X$ be a compact complex manifold equipped with a Hermitian metric $\omega$. Let $T=\alpha+i \partial \bar{\partial} \varphi$ be a closed $(1,1)$-current on $X$, where $\alpha$ is smooth and $\varphi$ is a quasi-plurisubharmonic function. Assume that $T \geqslant \gamma$ for some real $(1,1)$-form $\gamma$ on $X$ with real coefficients. Then there exists a sequence $T_{m}=\alpha+i \partial \bar{\partial} \varphi_{m}$ of closed $(1,1)$-currents such that

(a) $\varphi_{m}$ (and thus $T_{m}$ ) is smooth on the complement $X \backslash Z_{m}$ of an analytic set $Z_{m}$, and the $Z_{m}$ 's form an increasing sequence

$$
Z_{0} \subset Z_{1} \subset \cdots \subset Z_{m} \subset \cdots \subset X
$$

(b) There is a uniform estimate $T_{m} \geqslant \gamma-\delta_{m} \omega$ with $\lim \downarrow \delta_{m}=0$ as $m$ tends to $+\infty$.

(c) The sequence $\left(\varphi_{m}\right)$ is non increasing, and we have $\lim \downarrow \varphi_{m}=\varphi$. As a consequence, $T_{m}$ converges weakly to $T$ as $m$ tends to $+\infty$.

(d) Near $Z_{m}$, the potential $\varphi_{m}$ has logarithmic poles, namely, for every $x_{0} \in Z_{m}$, there is a neighborhood $U$ of $x_{0}$ such that $\varphi_{m}(z)=\lambda_{m} \log \sum_{\ell}\left|g_{m, \ell}\right|^{2}+O(1)$ for suitable holomorphic functions $\left(g_{m, \ell}\right)$ on $U$ and $\lambda_{m}>0$. Moreover, there is a (global) proper modification $\mu_{m}: \widetilde{X}_{m} \rightarrow X$ of $X$, obtained as a sequence of blow-ups with smooth centers, such that $\varphi_{m} \circ \mu_{m}$ can be written locally on $\widetilde{X}_{m}$ as

$$
\varphi_{m} \circ \mu_{m}(w)=\lambda_{m}\left(\sum n_{\ell} \log \left|\widetilde{g}_{\ell}\right|^{2}+f(w)\right)
$$

where $\left(\widetilde{g}_{\ell}=0\right)$ are local generators of suitable (global) divisors $E_{\ell}$ on $\widetilde{X}_{m}$ such that $\sum E_{\ell}$ has normal crossings, $n_{\ell}$ are positive integers, and the $f$ 's are smooth functions on $\tilde{X}_{m}$. 
Sketch of proof. We essentially repeat the proofs of Theorems 4.2 and 5.7 with additional considerations. One fact that does not follow readily from these proofs is the monotonicity of the sequence $\varphi_{m}$ (which we will not really need anyway - it can be obtained by applying Theorem 4.2 with $2^{m}$ instead of $m$, and by using the Ohsawa-Takegoshi $L^{2}$ extension theorem 4.1 for potentials $2^{m} \varphi(x)+2^{m} \varphi(y)$ on the diagonal of $X \times X$, so that the restriction is $2^{m+1} \varphi(x)$ on the diagonal; we refer e.g. to [DPS01] for details). The map $\mu_{m}$ is obtained by blowing-up the (global) ideals $\mathscr{F}_{m}$ defined by the holomorphic functions $\left(g_{j, m}\right)$ in the local approximations $\varphi_{m} \sim \frac{1}{2 m} \log \sum_{j}\left|g_{j, m}\right|^{2}$. By Hironaka [Hir64], we can achieve that $\mu_{m}^{*} \mathscr{f}_{m}$ is an invertible ideal sheaf associated with a normal crossing divisor.

(6.2) Corollary. If $T$ is a Kähler current, then one can write $T=\lim T_{m}$ for a sequence of Kähler currents $T_{m}$ which have logarithmic poles with coefficients in $\frac{1}{m} \mathbb{Z}$, i.e. there are modifications $\mu_{m}: X_{m} \rightarrow X$ such that

$$
\mu_{m}^{*} T_{m}=\left[E_{m}\right]+\beta_{m}
$$

where $E_{m}$ is an effective $\mathbb{Q}$-divisor on $X_{m}$ with coefficients in $\frac{1}{m} \mathbb{Z}$ (the "fixed part") and $\beta_{m}$ is a closed semi-positive form (the "mobile part").

Proof. We apply Theorem 6.1 with $\gamma=\varepsilon \omega$ and $m$ so large that $\delta_{m} \leqslant \varepsilon / 2$. Then $T_{m}$ has analytic singularities and $T_{m} \geqslant \frac{\varepsilon}{2} \omega$, so we get a composition of blow-ups $\mu_{m}: X_{m} \rightarrow X$ such

$$
\mu_{m}^{*} T_{m}=\left[E_{m}\right]+\beta_{m},
$$

where $E_{m}$ is an effective $\mathbb{Q}$-divisor and $\beta_{m} \geqslant \frac{\varepsilon}{2} \mu_{m}^{*} \omega$. In particular, $\beta_{m}$ is strictly positive outside the exceptional divisors, by playing with the multiplicities of the components of the exceptional divisors in $E_{m}$, we could even achieve that $\beta_{m}$ is a Kähler class on $X_{m}$. Notice also that by construction, $\mu_{m}$ is obtained by blowing-up the multiplier ideal sheaves $\mathscr{I}(m T)=\mathscr{F}(m \varphi)$ associated to a potential $\varphi$ of $T$.

The more familiar algebraic analogue would be to take $\alpha=c_{1}(L)$ with a big line bundle $L$ and to blow-up the base locus of $|m L|, m \gg 1$, to get a $\mathbb{Q}$-divisor decomposition

$$
\mu_{m}^{*} L \sim E_{m}+D_{m}, \quad E_{m} \text { effective, } D_{m} \text { base point free. }
$$

(One says that $D_{m}$ is base point free if $H^{0}\left(X, \mathscr{O}\left(D_{m}\right)\right.$ is generated by sections, in other words if $D_{m}$ is entirely "mobile" in the linear system $\left.\left|D_{m}\right|\right)$. Such a blow-up is usually referred to as a "log resolution" of the linear system $|m L|$, and we say that $E_{m}+D_{m}$ is an approximate Zariski decomposition of $L$. We will also use this terminology for Kähler currents with logarithmic poles.

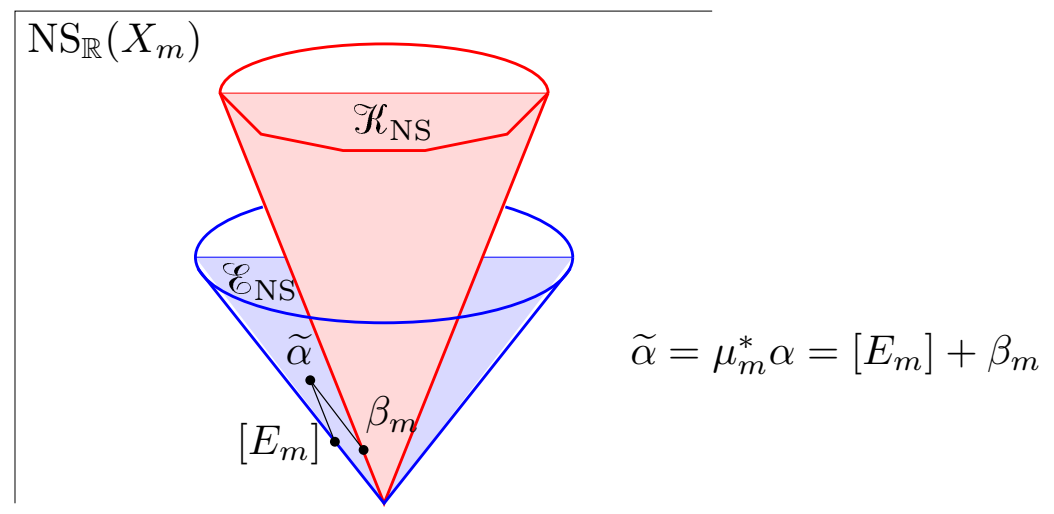


(6.4) Definition. We define the volume, or mobile self-intersection of a class $\alpha \in$ $H^{1,1}(X, \mathbb{R})$ to be

$$
\operatorname{Vol}(\alpha)=\sup _{T \in \alpha} \int_{X \backslash \operatorname{Sing}(T)} T^{n}=\sup _{T \in \alpha} \int_{\widetilde{X}} \beta^{n}>0
$$

where the supremum is taken over all Kähler currents $T \in \alpha$ with logarithmic poles, and $\mu^{*} T=[E]+\beta$ with respect to some modification $\mu: \widetilde{X} \rightarrow X$. Correspondingly, we set

$$
\operatorname{Vol}(\alpha)=0 \quad \text { if } \alpha \notin \mathscr{E}^{\circ} \text {. }
$$

In the special case where $\alpha=c_{1}(L)$ is an integral class, we have the following interpretation of the volume.

(6.5) Theorem. If $L$ is a big line bundle and $\mu_{m}^{*} L \sim E_{m}+D_{m}$ is a log resolution of $|m L|$, we have

$$
\operatorname{Vol}\left(c_{1}(L)\right)=\lim _{m \rightarrow+\infty} D_{m}^{n}=\lim _{m \rightarrow+\infty} \frac{n !}{m^{n}} h^{0}(X, m L)
$$

Sketch of proof. Given a Kähler current $T \in c_{1}(L)$ with logarithmic pole, we can always take a blow-up $\mu: \tilde{X} \rightarrow X$ so that $\mu^{*} T=[E]+\beta$ where $E$ is an effective $\mathbb{R}$-divisor and $\beta \geqslant 0$. By using a perturbation technique as in Lemma 5.13, we can always assume that $E$ is a $\mathbb{Q}$-divisor and that $\beta$ is Kähler. Then $\{\beta\}=\mu^{*} c_{1}(L)-\{[E]\}$ is a rational class and therefore $\beta$ is the first Chern class $c_{1}(A)$ of an ample $\mathbb{Q}$-divisor on $\widetilde{X}$. When $m$ is a multiple of a suitable denominator $m_{0}$ and $m=q m_{0}+r, 0 \leqslant r<m_{0}$, we get by the elementary Riemann-Roch formula

$$
h^{0}(X, m L) \geqslant h^{0}\left(\widetilde{X}, m \mu^{*} L-m_{0}\left[m / m_{0}\right] E\right)=h^{0}\left(\widetilde{X}, m_{0}\left[m / m_{0}\right] A+r \mu^{*} L\right) \sim \frac{m^{n}}{n !} \int_{\widetilde{X}} \beta^{n},
$$

hence $\lim \inf \frac{n !}{m^{n}} h^{0}(X, m L) \geqslant \operatorname{Vol}\left(c_{1}(L)\right)$ by taking the supremum over all such currents $T$. In the other direction, the inequality $\lim \sup \frac{n !}{m^{n}} h^{0}(X, m L) \leqslant \operatorname{Vol}\left(c_{1}(L)\right)$ is obtained by subtracting a small rational multiple $\varepsilon A$ of an ample line bundle $A$. One shows that multiples of $L-\varepsilon A$ roughly have the same number of sections as those of $L$ by an exact sequence argument similar to what was done in the proof of 3.4 (b). By a result of Fujita [Fuj94] (cf. also [DEL00]), the volume of the base point free part $D_{m, \varepsilon}$ in a $\log$ resolution of $|m(L-\varepsilon A)|$ approximates $\lim \sup \frac{n !}{m^{n}} h^{0}(X, m(L-\varepsilon A))$, so we get $\mu_{m, \varepsilon}^{*} L=E_{m, \varepsilon}+\left(D_{m, \varepsilon}+\varepsilon A\right)$ where $D_{m, \varepsilon}+A$ is ample. The positive $(1,1)$-current $T_{m, \varepsilon}=\left(\mu_{m, \varepsilon}\right)_{*} \Theta_{D_{m, \varepsilon}+\varepsilon A}$ is a Kähler current with logarithmic poles and its volume approaches $\lim \sup \frac{n !}{m^{n}} h^{0}(X, m L)$ when $\varepsilon \ll 1$ and $m$ is large.

In these terms, we get the following statement.

(6.6) Proposition. Let $L$ be a big line bundle on the projective manifold $X$. Let $\varepsilon>0$. Then there exists a modification $\mu: X_{\varepsilon} \rightarrow X$ and a decomposition $\mu^{*}(L)=E+\beta$ with $E$ an effective $\mathbb{Q}$-divisor and $\beta$ a big and nef $\mathbb{Q}$-divisor such that

$$
\operatorname{Vol}(L)-\varepsilon \leqslant \operatorname{Vol}(\beta) \leqslant \operatorname{Vol}(L) .
$$


It is very useful to observe that the supremum in Definition 6.4 is actually achieved by a collection of currents whose singularities satisfy a filtering property. Namely, if $T_{1}=\alpha+i \partial \bar{\partial} \varphi_{1}$ and $T_{2}=\alpha+i \partial \bar{\partial} \varphi_{2}$ are two Kähler currents with logarithmic poles in the class of $\alpha$, then

$$
T=\alpha+i \partial \bar{\partial} \varphi, \quad \varphi=\max \left(\varphi_{1}, \varphi_{2}\right)
$$

is again a Kähler current with weaker singularities than $T_{1}$ and $T_{2}$. One could define as well

$$
T=\alpha+i \partial \bar{\partial} \varphi, \quad \varphi=\frac{1}{2 m} \log \left(e^{2 m \varphi_{1}}+e^{2 m \varphi_{2}}\right)
$$

where $m=\operatorname{lcm}\left(m_{1}, m_{2}\right)$ is the lowest common multiple of the denominators occuring in $T_{1}, T_{2}$. Now, take a simultaneous log-resolution $\mu_{m}: X_{m} \rightarrow X$ for which the singularities of $T_{1}$ and $T_{2}$ are resolved as $\mathbb{Q}$-divisors $E_{1}$ and $E_{2}$. Then clearly the associated divisor in the decomposition $\mu_{m}^{*} T=[E]+\beta$ is given by $E=\min \left(E_{1}, E_{2}\right)$. By doing so, the volume $\int_{X_{m}} \beta^{n}$ gets increased, as we shall see in the proof of Theorem 6.8 below.

(6.8) Theorem (Boucksom [Bck02]). Let X be a compact Kähler manifold. We denote here by $H_{\geqslant 0}^{k, k}(X)$ the cone of cohomology classes of type $(k, k)$ which have non-negative intersection with all closed semi-positive smooth forms of bidegree $(n-k, n-k)$.

(a) For each integer $k=1,2, \ldots, n$, there exists a canonical "mobile intersection product"

$$
\mathscr{E} \times \cdots \times \mathscr{E} \rightarrow H_{\geqslant 0}^{k, k}(X), \quad\left(\alpha_{1}, \ldots, \alpha_{k}\right) \mapsto\left\langle\alpha_{1} \cdot \alpha_{2} \cdot \cdots \cdot \alpha_{k-1} \cdot \alpha_{k}\right\rangle
$$

such that $\operatorname{Vol}(\alpha)=\left\langle\alpha^{n}\right\rangle$ whenever $\alpha$ is a big class.

(b) The product is increasing, homogeneous of degree 1 and superadditive in each argument, i.e.

$$
\left\langle\alpha_{1} \cdots\left(\alpha_{j}^{\prime}+\alpha_{j}^{\prime \prime}\right) \cdots \alpha_{k}\right\rangle \geqslant\left\langle\alpha_{1} \cdots \alpha_{j}^{\prime} \cdots \alpha_{k}\right\rangle+\left\langle\alpha_{1} \cdots \alpha_{j}^{\prime \prime} \cdots \alpha_{k}\right\rangle
$$

It coincides with the ordinary intersection product when the $\alpha_{j} \in \overline{\mathscr{K}}$ are nef classes.

(c) The mobile intersection product satisfies the Hovanskii-Teissier inequalities ([Hov79], [Tei79, Tei82])

$$
\left\langle\alpha_{1} \cdot \alpha_{2} \cdot \cdots \cdot \alpha_{n}\right\rangle \geqslant\left(\left\langle\alpha_{1}^{n}\right\rangle\right)^{1 / n} \cdots\left(\left\langle\alpha_{n}^{n}\right\rangle\right)^{1 / n} \quad\left(\text { with }\left\langle\alpha_{j}^{n}\right\rangle=\operatorname{Vol}\left(\alpha_{j}\right)\right) .
$$

(d) For $k=1$, the above "product" reduces to a (non linear) projection operator

$$
\mathscr{E} \rightarrow \mathscr{E}_{1}, \quad \alpha \rightarrow\langle\alpha\rangle
$$

onto a certain convex subcone $\mathscr{E}_{1}$ of $\mathscr{E}$ such that $\overline{\mathscr{K}} \subset \mathscr{E}_{1} \subset \mathscr{E}$. Moreover, there is a "divisorial Zariski decomposition"

$$
\alpha=\{N(\alpha)\}+\langle\alpha\rangle
$$


where $N(\alpha)$ is a uniquely defined effective divisor which is called the "negative divisorial part" of $\alpha$. The map $\alpha \mapsto N(\alpha)$ is homogeneous and subadditive, and $N(\alpha)=0$ if and only if $\alpha \in \mathscr{E}_{1}$.

(e) The components of $N(\alpha)$ always consist of divisors whose cohomology classes are linearly independent, especially $N(\alpha)$ has at most $\rho=\operatorname{rank}_{\mathbb{Z}} \mathrm{NS}(X)$ components.

Proof. We essentially repeat the arguments developped in [Bck02], with some simplifications arising from the fact that $X$ is supposed to be Kähler from the beginning.

(a) First assume that all classes $\alpha_{j}$ are big, i.e. $\alpha_{j} \in \mathscr{E}^{\circ}$. Fix a smooth closed $(n-k, n-k)$ semi-positive form $u$ on $X$. We select Kähler currents $T_{j} \in \alpha_{j}$ with logarithmic poles, and a simultaneous log-resolution $\mu: \widetilde{X} \rightarrow X$ such that

$$
\mu^{*} T_{j}=\left[E_{j}\right]+\beta_{j}
$$

We consider the direct image current $\mu_{*}\left(\beta_{1} \wedge \cdots \wedge \beta_{k}\right)$ (which is a closed positive current of bidegree $(k, k)$ on $X)$ and the corresponding integrals

$$
\int_{\widetilde{X}} \beta_{1} \wedge \cdots \wedge \beta_{k} \wedge \mu^{*} u \geqslant 0
$$

If we change the representative $T_{j}$ with another current $T_{j}^{\prime}$, we may always take a simultaneous log-resolution such that $\mu^{*} T_{j}^{\prime}=\left[E_{j}^{\prime}\right]+\beta_{j}^{\prime}$, and by using $\left(6.7^{\prime}\right)$ we can always assume that $E_{j}^{\prime} \leqslant E_{j}$. Then $D_{j}=E_{j}-E_{j}^{\prime}$ is an effective divisor and we find $\left[E_{j}\right]+\beta_{j} \equiv\left[E_{j}^{\prime}\right]+\beta_{j}^{\prime}$, hence $\beta_{j}^{\prime} \equiv \beta_{j}+\left[D_{j}\right]$. A substitution in the integral implies

$$
\begin{aligned}
\int_{\widetilde{X}} \beta_{1}^{\prime} \wedge \beta_{2} & \wedge \cdots \wedge \beta_{k} \wedge \mu^{*} u \\
& =\int_{\widetilde{X}} \beta_{1} \wedge \beta_{2} \wedge \cdots \wedge \beta_{k} \wedge \mu^{*} u+\int_{\widetilde{X}}\left[D_{1}\right] \wedge \beta_{2} \wedge \cdots \wedge \beta_{k} \wedge \mu^{*} u \\
& \geqslant \int_{\widetilde{X}} \beta_{1} \wedge \beta_{2} \wedge \cdots \wedge \beta_{k} \wedge \mu^{*} u .
\end{aligned}
$$

Similarly, we can replace successively all forms $\beta_{j}$ by the $\beta_{j}^{\prime}$, and by doing so, we find

$$
\int_{\widetilde{X}} \beta_{1}^{\prime} \wedge \beta_{2}^{\prime} \wedge \cdots \wedge \beta_{k}^{\prime} \wedge \mu^{*} u \geqslant \int_{\widetilde{X}} \beta_{1} \wedge \beta_{2} \wedge \cdots \wedge \beta_{k} \wedge \mu^{*} u
$$

We claim that the closed positive currents $\mu_{*}\left(\beta_{1} \wedge \cdots \wedge \beta_{k}\right)$ are uniformly bounded in mass. In fact, if $\omega$ is a Kähler metric in $X$, there exists a constant $C_{j} \geqslant 0$ such that $C_{j}\{\omega\}-\alpha_{j}$ is a Kähler class. Hence $C_{j} \omega-T_{j} \equiv \gamma_{j}$ for some Kähler form $\gamma_{j}$ on $X$. By pulling back with $\mu$, we find $C_{j} \mu^{*} \omega-\left(\left[E_{j}\right]+\beta_{j}\right) \equiv \mu^{*} \gamma_{j}$, hence

$$
\beta_{j} \equiv C_{j} \mu^{*} \omega-\left(\left[E_{j}\right]+\mu^{*} \gamma_{j}\right)
$$

By performing again a substitution in the integrals, we find

$$
\int_{\widetilde{X}} \beta_{1} \wedge \cdots \wedge \beta_{k} \wedge \mu^{*} u \leqslant C_{1} \cdots C_{k} \int_{\widetilde{X}} \mu^{*} \omega^{k} \wedge \mu^{*} u=C_{1} \cdots C_{k} \int_{X} \omega^{k} \wedge u
$$


and this is true especially for $u=\omega^{n-k}$. We can now arrange that for each of the integrals associated with a countable dense family of forms $u$, the supremum is achieved by a sequence of currents $\left(\mu_{m}\right)_{*}\left(\beta_{1, m} \wedge \cdots \wedge \beta_{k, m}\right)$ obtained as direct images by a suitable sequence of modifications $\mu_{m}: \widetilde{X}_{m} \rightarrow X$. By extracting a subsequence, we can achieve that this sequence is weakly convergent and we set

$$
\left\langle\alpha_{1} \cdot \alpha_{2} \cdot \cdots \cdot \alpha_{k}\right\rangle=\lim _{m \rightarrow+\infty}\left\{\left(\mu_{m}\right)_{*}\left(\beta_{1, m} \wedge \beta_{2, m} \wedge \cdots \wedge \beta_{k, m}\right)\right\}
$$

(the monotonicity is not in terms of the currents themselves, but in terms of the integrals obtained when we evaluate against a smooth closed semi-positive form $u$ ). By evaluating against a basis of positive classes $\{u\} \in H^{n-k, n-k}(X)$, we infer by Serre duality that the class of $\left\langle\alpha_{1} \cdot \alpha_{2} \cdot \cdots \cdot \alpha_{k}\right\rangle$ is uniquely defined (although, in general, the representing current is not unique).

(b) It is indeed clear from the definition that the mobile intersection product is homogeneous, increasing and superadditive in each argument, at least when the $\alpha_{j}$ 's are in $\mathscr{E}^{\circ}$. However, we can extend the product to the closed cone $\mathscr{E}$ by monotonicity, by setting

$$
\left\langle\alpha_{1} \cdot \alpha_{2} \cdots \alpha_{k}\right\rangle=\lim _{\delta \downarrow 0} \downarrow\left\langle\left(\alpha_{1}+\delta \omega\right) \cdot\left(\alpha_{2}+\delta \omega\right) \cdot \cdots \cdot\left(\alpha_{k}+\delta \omega\right)\right\rangle
$$

for arbitrary classes $\alpha_{j} \in \mathscr{E}$ (again, monotonicity occurs only where we evaluate against closed semi-positive forms $u$ ). By weak compactness, the mobile intersection product can always be represented by a closed positive current of bidegree $(k, k)$.

(c) The Hovanskii-Teissier inequalities are a direct consequence of the fact that they hold true for nef classes, so we just have to apply them to the classes $\beta_{j, m}$ on $\widetilde{X}_{m}$ and pass to the limit.

(d) When $k=1$ and $\alpha \in \mathscr{E}^{0}$, we have

$$
\alpha=\lim _{m \rightarrow+\infty}\left\{\left(\mu_{m}\right)_{*} T_{m}\right\}=\lim _{m \rightarrow+\infty}\left(\mu_{m}\right)_{*}\left[E_{m}\right]+\left\{\left(\mu_{m}\right)_{*} \beta_{m}\right\}
$$

and $\langle\alpha\rangle=\lim _{m \rightarrow+\infty}\left\{\left(\mu_{m}\right)_{*} \beta_{m}\right\}$ by definition. However, the images $F_{m}=\left(\mu_{m}\right)_{*}$ $F_{m}$ are effective $\mathbb{Q}$-divisors in $X$, and the filtering property implies that $F_{m}$ is a decreasing sequence. It must therefore converge to a (uniquely defined) $\operatorname{limit} F=\lim F_{m}:=N(\alpha)$ which is an effective $\mathbb{R}$-divisor, and we get the asserted decomposition in the limit.

Since $N(\alpha)=\alpha-\langle\alpha\rangle$ we easily see that $N(\alpha)$ is subadditive and that $N(\alpha)=0$ if $\alpha$ is the class of a smooth semi-positive form. When $\alpha$ is no longer a big class, we define

$$
\langle\alpha\rangle=\lim _{\delta \downarrow 0} \downarrow\langle\alpha+\delta \omega\rangle, \quad N(\alpha)=\lim _{\delta \downarrow 0} \uparrow N(\alpha+\delta \omega)
$$

(the subadditivity of $N$ implies $N(\alpha+(\delta+\varepsilon) \omega) \leqslant N(\alpha+\delta \omega)$ ). The divisorial Zariski decomposition follows except maybe for the fact that $N(\alpha)$ might be a convergent countable sum of divisors. However, this will be ruled out when (e) is proved. As $N(\bullet)$ is subadditive and homogeneous, the set $\mathscr{E}_{1}=\{\alpha \in \mathscr{E} ; N(\alpha)=0\}$ is a closed convex cone, and we find that $\alpha \mapsto\langle\alpha\rangle$ is a projection of $\mathscr{E}_{\text {onto }} \mathscr{E}_{1}$ (according to [Bck02], $\mathscr{E}_{1}$ consists of those pseudo-effective classes which are "nef in codimension 1"). 
(e) Let $\alpha \in \mathscr{E}^{\circ}$, and assume that $N(\alpha)$ contains linearly dependent components $F_{j}$. Then already all currents $T \in \alpha$ should be such that $\mu^{*} T=[E]+\beta$ where $F=\mu_{*} E$ contains those linearly dependent components. Write $F=\sum \lambda_{j} F_{j}, \lambda_{j}>0$ and assume that

$$
\sum_{j \in J} c_{j} F_{j} \equiv 0
$$

for a certain non trivial linear combination. Then some of the coefficients $c_{j}$ must be negative (and some other positive). Then $E$ is numerically equivalent to

$$
E^{\prime} \equiv E+t \mu^{*}\left(\sum \lambda_{j} F_{j}\right)
$$

and by choosing $t>0$ appropriate, we obtain an effective divisor $E^{\prime}$ which has a zero coefficient on one of the components $\mu^{*} F_{j_{0}}$. By replacing $E$ with $\min \left(E, E^{\prime}\right)$ via $\left(6.7^{\prime}\right)$, we eliminate the component $\mu^{*} F_{j_{0}}$. This is a contradiction since $N(\alpha)$ was supposed to contain $F_{j_{0}}$.

(6.9) Definition. For a class $\alpha \in H^{1,1}(X, \mathbb{R})$, we define the numerical dimension nd $(\alpha)$ to be $\operatorname{nd}(\alpha)=-\infty$ if $\alpha$ is not pseudo-effective, and

$$
\operatorname{nd}(\alpha)=\max \left\{p \in \mathbb{N} ;\left\langle\alpha^{p}\right\rangle \neq 0\right\}, \quad \operatorname{nd}(\alpha) \in\{0,1, \ldots, n\}
$$

if $\alpha$ is pseudo-effective.

By the results of [DP04], a class is big $\left(\alpha \in \mathscr{E}^{\circ}\right)$ if and only if $\operatorname{nd}(\alpha)=n$. Classes of numerical dimension 0 can be described much more precisely, again following Boucksom [Bck02].

(6.10) Theorem. Let $X$ be a compact Kähler manifold. Then the subset $\mathscr{D}_{0}$ of irreducible divisors $D$ in $X$ such that $\operatorname{nd}(D)=0$ is countable, and these divisors are rigid as well as their multiples. If $\alpha \in \mathscr{E}$ is a pseudo-effective class of numerical dimension 0 , then $\alpha$ is numerically equivalent to an effective $\mathbb{R}$-divisor $D=\sum_{j \in J} \lambda_{j} D_{j}$, for some finite subset $\left(D_{j}\right)_{j \in J} \subset \mathscr{D}_{0}$ such that the cohomology classes $\left\{D_{j}\right\}$ are linearly independent and some $\lambda_{j}>0$. If such a linear combination is of numerical dimension 0 , then so is any other linear combination of the same divisors.

Proof. It is immediate from the definition that a pseudo-effective class is of numerical dimension 0 if and only if $\langle\alpha\rangle=0$, in other words if $\alpha=N(\alpha)$. Thus $\alpha \equiv \sum \lambda_{j} D_{j}$ as described in 6.10, and since $\lambda_{j}\left\langle D_{j}\right\rangle \leqslant\langle\alpha\rangle$, the divisors $D_{j}$ must themselves have numerical dimension 0 . There is at most one such divisor $D$ in any given cohomology class in $N S(X) \cap \mathscr{E} \subset H^{2}(X, \mathbb{Z})$, otherwise two such divisors $D \equiv D^{\prime}$ would yield a blow-up $\mu: \widetilde{X} \rightarrow X$ resolving the intersection, and by taking $\min \left(\mu^{*} D, \mu^{*} D^{\prime}\right)$ via $\left(6.7^{\prime}\right)$, we would find $\mu^{*} D \equiv E+\beta, \beta \neq 0$, so that $\{D\}$ would not be of numerical dimension 0 . This implies that there are at most countably many divisors of numerical dimension 0 , and that these divisors are rigid as well as their multiples.

(6.11) Remark. If $L$ is an arbitrary holomorphic line bundle, we define its numerical dimension to be $\operatorname{nd}(L)=\operatorname{nd}\left(c_{1}(L)\right)$. Using the canonical maps $\Phi_{|m L|}$ and pulling-back the Fubini-Study metric it is immediate to see that $\operatorname{nd}(L) \geqslant \kappa(L)$. 
The above general concept of numerical dimension leads to a very natural formulation of the abundance conjecture for Kähler varieties.

(6.12) Generalized Abundance Conjecture. Let $X$ be an arbitrary compact Kähler manifold $X$.

(a) The Kodaira dimension of $X$ should be equal to its numerical dimension: $\kappa\left(K_{X}\right)=$ $\operatorname{nd}\left(K_{X}\right)$.

(b) More generally, let $\Delta$ be a $\mathbb{Q}$-divisor which is klt (Kawamata log terminal, i.e. such that $\left.c_{X}(\Delta)>1\right)$. Then $\kappa\left(K_{X}+\Delta\right)=\operatorname{nd}\left(K_{X}+\Delta\right)$.

(6.13) Remark. It is obvious that abundance holds in the case $\operatorname{nd}\left(K_{X}\right)=-\infty$ (if $L$ is not pseudo-effective, no multiple of $L$ can have sections), or in the case $\operatorname{nd}\left(K_{X}\right)=n$ which implies $K_{X}$ big (the latter property follows e.g. from the solution of the GrauertRiemenschneider conjecture in the form proven in [Dem85], see also [DP04]).

In the remaining cases, the most tractable situation is the case when $\mathrm{nd}\left(K_{X}\right)=0$. In fact Theorem 6.10 then gives $K_{X} \equiv \sum \lambda_{j} D_{j}$ for some effective divisor with numerically independent components, $\operatorname{nd}\left(D_{j}\right)=0$. It follows that the $\lambda_{j}$ are rational and therefore

$$
K_{X} \sim \sum \lambda_{j} D_{j}+F \quad \text { where } \lambda_{j} \in \mathbb{Q}^{+}, \operatorname{nd}\left(D_{j}\right)=0 \text { and } F \in \operatorname{Pic}^{0}(X) .
$$

If we assume additionally that $q(X)=h^{0,1}(X)$ is zero, then $m K_{X}$ is linearly equivalent to an integral divisor for some multiple $m$, and it follows immediately that $\kappa(X)=0$. The case of a general projective manifold with $\operatorname{nd}\left(K_{X}\right)=0$ and positive irregularity $q(X)>0$ has been solved by Campana-Peternell [CP04], Proposition 3.7. It would be interesting to understand the Kähler case as well.

\section{The orthogonality estimate}

The goal of this section is to show that, in an appropriate sense, approximate Zariski decompositions are almost orthogonal.

(7.1) Theorem. Let $X$ be a projective manifold, and let $\alpha=\{T\} \in \mathscr{E}_{\mathrm{NS}}^{\circ}$ be a big class represented by a Kähler current $T$. Consider an approximate Zariski decomposition

$$
\mu_{m}^{*} T_{m}=\left[E_{m}\right]+\left[D_{m}\right]
$$

Then

$$
\left(D_{m}^{n-1} \cdot E_{m}\right)^{2} \leqslant 20(C \omega)^{n}\left(\operatorname{Vol}(\alpha)-D_{m}^{n}\right)
$$

where $\omega=c_{1}(H)$ is a Kähler form and $C \geqslant 0$ is a constant such that $\pm \alpha$ is dominated by $C \omega$ (i.e., $C \omega \pm \alpha$ is nef). In other words, $E_{m}$ and $D_{m}$ become "more and more orthogonal" as $D_{m}^{n}$ approaches the volume.

Proof. For every $t \in[0,1]$, we have

$$
\operatorname{Vol}(\alpha)=\operatorname{Vol}\left(E_{m}+D_{m}\right) \geqslant \operatorname{Vol}\left(t E_{m}+D_{m}\right)
$$


Now, by our choice of $C$, we can write $E_{m}$ as a difference of two nef divisors

$$
E_{m}=\mu^{*} \alpha-D_{m}=\mu_{m}^{*}(\alpha+C \omega)-\left(D_{m}+C \mu_{m}^{*} \omega\right)
$$

(7.2) Lemma. For all nef $\mathbb{R}$-divisors $A, B$ we have

$$
\operatorname{Vol}(A-B) \geqslant A^{n}-n A^{n-1} \cdot B
$$

as soon as the right hand side is positive.

Proof. In case $A$ and $B$ are integral divisors, this is a consequence of holomorphic Morse inequalities (cf. (I 2.15)). If $A$ and $B$ are $\mathbb{Q}$-divisors, we conclude by the homogeneity of the volume. The general case of $\mathbb{R}$-divisors follows by approximation (actually, as it is defined to be a supremum, the volume function can easily be shown to be lower semi-continuous, but it is in fact even continuous, cf. [Bck02, 3.1.26]).

(7.3) Remark. We hope that Lemma 7.2 also holds true on an arbitrary Kähler manifold for arbitrary nef (non necessarily integral) classes. This would follow from Conjecture (III 2.11) generalizing holomorphic Morse inequalities to non integral classes, exactly by the same proof as Theorem (I 2.14).

(7.4) Lemma. Let $\beta_{1}, \ldots, \beta_{n}$ and $\beta_{1}^{\prime}, \ldots, \beta_{n}^{\prime}$ be nef classes on a compact Kähler manifold $\widetilde{X}$ such that each difference $\beta_{j}^{\prime}-\beta_{j}$ is pseudo-effective. Then the $n$-th intersection products satisfy

$$
\beta_{1} \cdots \beta_{n} \leqslant \beta_{1}^{\prime} \cdots \beta_{n}^{\prime}
$$

Proof. We can proceed step by step and replace just one $\beta_{j}$ by $\beta^{\prime} j \equiv \beta_{j}+T_{j}$ where $T_{j}$ is a closed positive $(1,1)$-current and the other classes $\beta_{k}^{\prime}=\beta_{k}, k \neq j$ are limits of Kähler forms. The inequality is then obvious.

End of proof of Theorem 7.1. In order to exploit the lower bound of the volume, we write

$$
t E_{m}+D_{m}=A-B, \quad A=D_{m}+t \mu_{m}^{*}(\alpha+C \omega), \quad B=t\left(D_{m}+C \mu_{m}^{*} \omega\right) .
$$

By our choice of the constant $C$, both $A$ and $B$ are nef. Lemma 7.2 and the binomial formula imply

$$
\begin{aligned}
\operatorname{Vol}\left(t E_{m}+D_{m}\right) \geqslant & A^{n}-n A^{n-1} \cdot B \\
=D_{m}^{n} & +n t D_{m}^{n-1} \cdot \mu_{m}^{*}(\alpha+C \omega)+\sum_{k=2}^{n} t^{k}\left(\begin{array}{l}
n \\
k
\end{array}\right) D_{m}^{n-k} \cdot \mu_{m}^{*}(\alpha+C \omega)^{k} \\
& -n t D_{m}^{n-1} \cdot\left(D_{m}+C \mu_{m}^{*} \omega\right) \\
& -n t^{2} \sum_{k=1}^{n-1} t^{k-1}\left(\begin{array}{c}
n-1 \\
k
\end{array}\right) D_{m}^{n-1-k} \cdot \mu_{m}^{*}(\alpha+C \omega)^{k} \cdot\left(D_{m}+C \mu_{m}^{*} \omega\right) .
\end{aligned}
$$

Now, we use the obvious inequalities

$$
D_{m} \leqslant \mu_{m}^{*}(C \omega), \quad \mu_{m}^{*}(\alpha+C \omega) \leqslant 2 \mu_{m}^{*}(C \omega), \quad D_{m}+C \mu_{m}^{*} \omega \leqslant 2 \mu_{m}^{*}(C \omega)
$$


in which all members are nef (and where the inequality $\leqslant$ means that the difference of classes is pseudo-effective). We use Lemma 7.4 to bound the last summation in the estimate of the volume, and in this way we get

$$
\operatorname{Vol}\left(t E_{m}+D_{m}\right) \geqslant D_{m}^{n}+n t D_{m}^{n-1} \cdot E_{m}-n t^{2} \sum_{k=1}^{n-1} 2^{k+1} t^{k-1}\left(\begin{array}{c}
n-1 \\
k
\end{array}\right)(C \omega)^{n}
$$

We will always take $t$ smaller than $1 / 10 n$ so that the last summation is bounded by $4(n-1)(1+1 / 5 n)^{n-2}<4 n e^{1 / 5}<5 n$. This implies

$$
\operatorname{Vol}\left(t E_{m}+D_{m}\right) \geqslant D_{m}^{n}+n t D_{m}^{n-1} \cdot E_{m}-5 n^{2} t^{2}(C \omega)^{n} .
$$

Now, the choice $t=\frac{1}{10 n}\left(D_{m}^{n-1} \cdot E_{m}\right)\left((C \omega)^{n}\right)^{-1}$ gives by substituting

$$
\frac{1}{20} \frac{\left(D_{m}^{n-1} \cdot E_{m}\right)^{2}}{(C \omega)^{n}} \leqslant \operatorname{Vol}\left(E_{m}+D_{m}\right)-D_{m}^{n} \leqslant \operatorname{Vol}(\alpha)-D_{m}^{n}
$$

(and we have indeed $t \leqslant \frac{1}{10 n}$ by Lemma 7.4), whence Theorem 7.1. Of course, the constant 20 is certainly not optimal.

(7.5) Corollary. If $\alpha \in \mathscr{E}_{\mathrm{NS}}$, then the divisorial Zariski decomposition $\alpha=N(\alpha)+\langle\alpha\rangle$ is such that

$$
\left\langle\alpha^{n-1}\right\rangle \cdot N(\alpha)=0
$$

Proof. By replacing $\alpha$ with $\alpha+\delta c_{1}(H)$, one sees that it is sufficient to consider the case where $\alpha$ is big. Then the orthogonality estimate implies

$$
\begin{aligned}
\left(\mu_{m}\right)_{*}\left(D_{m}^{n-1}\right) \cdot\left(\mu_{m}\right)_{*} E_{m} & =D_{m}^{n-1} \cdot\left(\mu_{m}\right)^{*}\left(\mu_{m}\right)_{*} E_{m} \\
& \leqslant D_{m}^{n-1} \cdot E_{m} \leqslant C\left(\operatorname{Vol}(\alpha)-D_{m}^{n}\right)^{1 / 2} .
\end{aligned}
$$

Since $\left\langle\alpha^{n-1}\right\rangle=\lim \left(\mu_{m}\right)_{*}\left(D_{m}^{n-1}\right), N(\alpha)=\lim \left(\mu_{m}\right)_{*} E_{m}$ and $\lim D_{m}^{n}=\operatorname{Vol}(\alpha)$, we get the desired conclusion in the limit.

\section{Dual of the pseudo-effective cone}

We consider here the Serre duality pairing

$$
H^{1,1}(X, \mathbb{R}) \times H^{n-1, n-1}(X, \mathbb{R}) \longrightarrow \mathbb{R}, \quad(\alpha, \beta) \longmapsto \alpha \cdot \beta=\int_{X} \alpha \wedge \beta .
$$

When restricted to real vector subspaces generated by integral classes, it defines a perfect pairing

$$
\mathrm{NS}_{\mathbb{R}} \times \mathrm{NS}_{\mathbb{R}}^{n-1, n-1}(X) \longrightarrow \mathbb{R}
$$

where $\mathrm{NS}_{\mathbb{R}} \subset H^{1,1}(X, \mathbb{R})$ and $\mathrm{NS}_{\mathbb{R}}^{n-1, n-1}(X) \subset H^{n-1, n-1}(X, \mathbb{R})$. Next, we introduce the concept of mobile curves. 
(8.3) Definition. Let $X$ be a smooth projective variety.

(a) One defines $\mathrm{NE}(X) \subset \mathrm{NS}_{\mathbb{R}}^{n-1, n-1}(X)$ to be the convex cone generated by cohomology classes of all effective curves in $H^{n-1, n-1}(X, \mathbb{R})$.

(b) We say that $C$ is a mobile curve if $C=C_{t_{0}}$ is a member of an analytic family $\left\{C_{t}\right\}_{t \in S}$ such that $\bigcup_{t \in S} C_{t}=X$ and, as such, is a reduced irreducible 1-cycle. We define the mobile cone $\mathrm{ME}(X)$, to be the convex cone generated by all mobile curves.

(c) If $X$ is projective, we say that an effective 1-cycle $C$ is a strongly mobile if we have

$$
C=\mu_{*}\left(\widetilde{A}_{1} \cap \cdots \cap \widetilde{A}_{n-1}\right)
$$

for suitable very ample divisors $\widetilde{A}_{j}$ on $\tilde{X}$, where $\mu: \widetilde{X} \rightarrow X$ is a modification. We let $\mathrm{ME}^{s}(X)$ be the convex cone generated by all strongly mobile effective 1-cycles (notice that by taking $\widetilde{A}_{j}$ general enough these classes can be represented by reduced irreducible curves; also, by Hironaka, one could just restrict oneself to compositions of blow-ups with smooth centers).

Clearly, we have

$$
\operatorname{ME}^{s}(X) \subset \mathrm{ME}(X) \subset \mathrm{NE}(X) \subset \mathrm{NS}_{\mathbb{R}}^{n-1, n-1}(X) .
$$

Another simple observation is:

(8.5) Proposition. One has $\alpha \cdot C \geqslant 0$ whenever $\{\alpha\} \in \mathscr{E}$ and $\{C\} \in \operatorname{ME}(X)$. In other words $\mathscr{E}_{\mathrm{NS}}=\mathscr{E} \cap \mathrm{NS}_{\mathbb{R}}(X)$ is contained in the dual cone $(\mathrm{ME}(X))^{\vee}$.

Proof. If the class $\{\alpha\}$ is represented by a closed positive current $T$ and $C=C_{t_{0}}$ belongs to a covering family $\left(C_{t}\right)_{t \in S}$, it is easy to see that $T_{\mid C_{t}}$ is locally well defined and nonnegative as soon as $C_{t}$ is not contained in the set of poles of a local potential $\varphi$ of $T$. However, this occurs only when $t$ belongs to a pluripolar set $P \subset S$, hence for $t \in S \backslash P$ we have

$$
\alpha \cdot C=\int_{C_{t}} T_{\mid C_{t}} \geqslant 0
$$

The following statement was first proved in [BDPP04].

(8.6) Theorem. If $X$ is projective, the cones $\mathscr{E}_{\mathrm{NS}}=\overline{\mathrm{Eff}(X)}$ and $\overline{\mathrm{ME}^{s}(X)}$ are dual with respect to Serre duality, and we have $\overline{\mathrm{ME}^{s}(X)}=\overline{\mathrm{ME}(X)}$.

In other words, a line bundle $L$ is pseudo-effective if (and only if) $L \cdot C \geqslant 0$ for all mobile curves, i.e., $L \cdot C \geqslant 0$ for every very generic curve $C$ (not contained in a countable union of algebraic subvarieties). In fact, by definition of $\operatorname{ME}^{s}(X)$, it is enough to consider only those curves $C$ which are images of generic complete intersection of very ample divisors on some variety $\widetilde{X}$, under a modification $\mu: \widetilde{X} \rightarrow X$. By a standard blowing-up argument, it also follows that a line bundle $L$ on a normal Moishezon variety is pseudo-effective if and only if $L \cdot C \geqslant 0$ for every mobile curve $C$. 
Proof. By (8.5) we have $\mathscr{E}_{\mathrm{NS}} \subset(\mathrm{ME}(X))^{\vee}$ and (8.4) implies $(\mathrm{ME}(X))^{\vee} \subset\left(\mathrm{ME}^{s}(X)\right)^{\vee}$, therefore

$$
\mathscr{E}_{\mathrm{NS}} \subset\left(\mathrm{ME}^{s}(X)\right)^{\vee}
$$

If we show that $\mathscr{E}_{\mathrm{NS}}=\left(\mathrm{ME}^{s}(X)\right)^{\vee}$, we get at the same time $\left(\mathrm{ME}^{s}(X)\right)^{\vee}=(\operatorname{ME}(X))^{\vee}$, and therefore by biduality (Hahn-Banach theorem) we will infer $\overline{\operatorname{ME}^{s}(X)}=\overline{\operatorname{ME}(X)}$. Now, if the inclusion were strict in (8.7), there would be an element $\alpha \in \partial \mathscr{E}_{\mathrm{NS}}$ on the boundary of $\mathscr{E}_{\mathrm{NS}}$ which is in the interior of $\mathrm{ME}^{s}(X)^{\vee}$.
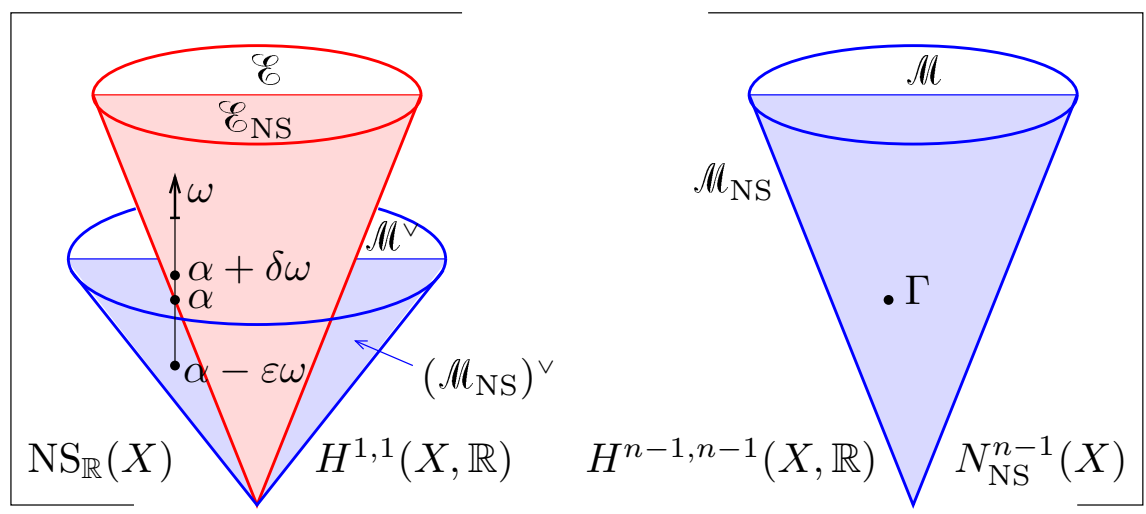

Let $\omega=c_{1}(H)$ be an ample class. Since $\alpha \in \partial \mathscr{E}_{\mathrm{NS}}$, the class $\alpha+\delta \omega$ is big for every $\delta>0$, and since $\alpha \in\left(\left(\operatorname{ME}^{s}(X)\right)^{\vee}\right)^{\circ}$ we still have $\alpha-\varepsilon \omega \in\left(\operatorname{ME}^{s}(X)\right)^{\vee}$ for $\varepsilon>0$ small. Therefore

$$
\alpha \cdot \Gamma \geqslant \varepsilon \omega \cdot \Gamma
$$

for every strongly mobile curve $\Gamma$, and therefore for every $\Gamma \in \overline{\operatorname{ME}^{s}(X)}$. We are going to contradict (8.8). Since $\alpha+\delta \omega$ is big, we have an approximate Zariski decomposition

$$
\mu_{\delta}^{*}(\alpha+\delta \omega)=E_{\delta}+D_{\delta}
$$

We pick $\Gamma=\left(\mu_{\delta}\right)_{*}\left(D_{\delta}^{n-1}\right) \in \overline{\mathrm{ME}^{s}(X)}$. By the Hovanskii-Teissier concavity inequality

$$
\omega \cdot \Gamma \geqslant\left(\omega^{n}\right)^{1 / n}\left(D_{\delta}^{n}\right)^{(n-1) / n} .
$$

On the other hand

$$
\begin{aligned}
\alpha \cdot \Gamma & =\alpha \cdot\left(\mu_{\delta}\right)_{*}\left(D_{\delta}^{n-1}\right) \\
& =\mu_{\delta}^{*} \alpha \cdot D_{\delta}^{n-1} \leqslant \mu_{\delta}^{*}(\alpha+\delta \omega) \cdot D_{\delta}^{n-1} \\
& =\left(E_{\delta}+D_{\delta}\right) \cdot D_{\delta}^{n-1}=D_{\delta}^{n}+D_{\delta}^{n-1} \cdot E_{\delta} .
\end{aligned}
$$

By the orthogonality estimate, we find

$$
\begin{aligned}
\frac{\alpha \cdot \Gamma}{\omega \cdot \Gamma} & \leqslant \frac{D_{\delta}^{n}+\left(20(C \omega)^{n}\left(\operatorname{Vol}(\alpha+\delta \omega)-D_{\delta}^{n}\right)\right)^{1 / 2}}{\left(\omega^{n}\right)^{1 / n}\left(D_{\delta}^{n}\right)^{(n-1) / n}} \\
& \leqslant C^{\prime}\left(D_{\delta}^{n}\right)^{1 / n}+C^{\prime \prime} \frac{\left(\operatorname{Vol}(\alpha+\delta \omega)-D_{\delta}^{n}\right)^{1 / 2}}{\left(D_{\delta}^{n}\right)^{(n-1) / n}}
\end{aligned}
$$


However, since $\alpha \in \partial \mathscr{E}_{\mathrm{NS}}$, the class $\alpha$ cannot be big so

$$
\lim _{\delta \rightarrow 0} D_{\delta}^{n}=\operatorname{Vol}(\alpha)=0
$$

We can also take $D_{\delta}$ to approximate $\operatorname{Vol}(\alpha+\delta \omega)$ in such a way that $\left(\operatorname{Vol}(\alpha+\delta \omega)-D_{\delta}^{n}\right)^{1 / 2}$ tends to 0 much faster than $D_{\delta}^{n}$. Notice that $D_{\delta}^{n} \geqslant \delta^{n} \omega^{n}$, so in fact it is enough to take

$$
\operatorname{Vol}(\alpha+\delta \omega)-D_{\delta}^{n} \leqslant \delta^{2 n}
$$

which gives $(\alpha \cdot \Gamma) /(\omega \cdot \Gamma) \leqslant\left(C^{\prime}+C^{\prime \prime}\right) \delta$. This contradicts (8.8) for $\delta$ small. 


\section{Part III}

\section{Asymptotic cohomology functionals and Monge-Ampère operators}

The goal of this chapter is to show that there are strong relations between certain Monge-Ampère integrals appearing in holomorphic Morse inequalities, and asymptotic cohomology estimates for tensor powers of holomorphic line bundles. Especially, we prove that these relations hold without restriction for projective surfaces, and in the special case of the volume, i.e. of asymptotic 0-cohomology, for all projective manifolds. These results can be seen as a partial converse to the Andreotti-Grauert vanishing theorem.

\section{Introduction and main definitions}

Throughout this chapter, $X$ denotes a compact complex manifold, $n=\operatorname{dim}_{\mathbb{C}} X$ its complex dimension and $L \rightarrow X$ a holomorphic line bundle. In order to estimate the growth of cohomology groups, it is interesting to consider appropriate "asymptotic cohomology functions". Following partly notation and concepts introduced by A. Küronya [Kür06, FKL07], we introduce

(0.1) Definition. Let $X$ be a compact complex manifold and let $L \rightarrow X$ be a holomorphic line bundle.

(a) The q-th asymptotic cohomology functional is defined as

$$
\widehat{h}^{q}(X, L):=\limsup _{k \rightarrow+\infty} \frac{n !}{k^{n}} h^{q}\left(X, L^{\otimes k}\right) .
$$

(b) The q-th asymptotic holomorphic Morse sum of $L$ is

$$
\widehat{h}^{\leq q}(X, L):=\limsup _{k \rightarrow+\infty} \frac{n !}{k^{n}} \sum_{0 \leqslant j \leqslant q}(-1)^{q-j} h^{j}\left(X, L^{\otimes k}\right) .
$$

When the limsup's are limits, we have the obvious relation

$$
\widehat{h}^{\leq q}(X, L)=\sum_{0 \leqslant j \leqslant q}(-1)^{q-j} \widehat{h}^{j}(X, L) .
$$

Clearly, Definition 0.1 can also be given for a $\mathbb{Q}$-line bundle $L$ or a $\mathbb{Q}$-divisor $D$, and in the case $q=0$ one gets by (II 6.5) what is called the volume of $L$ (see also [DEL00], [Bck02], [Laz04]):

$$
\operatorname{Vol}(X, L):=\widehat{h}^{0}(X, L)=\limsup _{k \rightarrow+\infty} \frac{n !}{k^{n}} h^{0}\left(X, L^{\otimes k}\right) .
$$




\section{Extension of the functionals to real cohomology classes}

We are going to show that the $\widehat{h}^{q}$ functional induces a continuous map

$$
\operatorname{DNS}_{\mathbb{R}}(X) \ni \alpha \mapsto \widehat{h}_{\mathrm{DNS}}^{q}(X, \alpha),
$$

which is defined on the "divisorial Néron-Severi space" $\operatorname{DNS}_{\mathbb{R}}(X) \subset H_{\mathrm{BC}}^{1,1}(X, \mathbb{R})$, i.e. the vector space spanned by real linear combinations of classes of divisors in the real Bott-Chern cohomology group of bidegree $(1,1)$. Here $H_{\mathrm{BC}}^{p, q}(X, \mathbb{C})$ is defined as the quotient of $d$-closed $(p, q)$-forms by $\partial \bar{\partial}$-exact $(p, q)$-forms, and there is a natural conjugation $H_{\mathrm{BC}}^{p, q}(X, \mathbb{C}) \rightarrow H_{\mathrm{BC}}^{q, p}(X, \mathbb{C})$ which allows us to speak of real classes when $q=p$. Notice that $H_{\mathrm{BC}}^{p, q}(X, \mathbb{C})$ coincides with the usual Dolbeault cohomology group $H^{p, q}(X, \mathbb{C})$ when $X$ is Kähler, and that $\operatorname{DNS}_{\mathbb{R}}(X)$ coincides with the usual Néron-Severi space

$$
\mathrm{NS}_{\mathbb{R}}(X)=\mathbb{R} \otimes_{\mathbb{Q}}\left(H^{2}(X, \mathbb{Q}) \cap H^{1,1}(X, \mathbb{C})\right)
$$

when $X$ is projective (the inclusion can be strict in general, e.g. on complex 2-tori which only have indefinite integral $(1,1)$-classes, cf. [BL04]).

For $\alpha \in \mathrm{NS}_{\mathbb{R}}(X)$ (resp. $\alpha \in \mathrm{DNS}_{\mathbb{R}}(X)$ ), we set

$$
\begin{aligned}
\widehat{h}_{\mathrm{NS}}^{q}(X, \alpha) \quad\left(\operatorname{resp} . \widehat{h}_{\mathrm{DNS}}^{q}(X, \alpha)\right) & =\limsup _{k \rightarrow+\infty, \frac{1}{k} c_{1}(L) \rightarrow \alpha} \frac{n !}{k^{n}} h^{q}(X, L) \\
& =\inf _{\varepsilon>0, k_{0}>0} \sup _{k \geqslant k_{0},\left\|\frac{1}{k} c_{1}(L)-\alpha\right\| \leqslant \varepsilon} \frac{n !}{k^{n}} h^{q}(X, L) .
\end{aligned}
$$

when the pair $(k, L)$ runs over $\mathbb{N}^{*} \times \operatorname{Pic}(X)$, resp. over $\mathbb{N}^{*} \times \operatorname{Pic}_{D}(X)$ where $\operatorname{Pic}_{D}(X) \subset$ $\operatorname{Pic}(X)$ is the subgroup generated by "divisorial line bundles", i.e. line bundles of the form $\sigma_{X}(D)$. Similar definitions can be given for the Morse sum functionals $\widehat{h}_{\mathrm{NS}}^{\leqslant q}(X, \alpha)$ and $\widehat{h}_{\mathrm{DNS}}^{\leqslant q}(X, \alpha)$. Clearly $\widehat{h}_{\mathrm{DNS}}^{\leqslant q}(X, \alpha) \leqslant \widehat{h}_{\mathrm{NS}}^{\leqslant q}(X, \alpha)$ on $\operatorname{DNS}_{\mathbb{R}}(X)$, but we do not know at this point whether this is always an equality. From the very definition, $\widehat{h}_{\mathrm{NS}}^{q}, \widehat{h}_{\mathrm{NS}}^{\leqslant q}$ (and likewise $\widehat{h}_{\mathrm{DNS}}^{q}, \widehat{h}_{\mathrm{DNS}}^{\leqslant q}$ ) are upper semi-continuous functions which are positively homogeneous of degree $n$, namely

$$
\widehat{h}_{\mathrm{NS}}^{q}(X, \lambda \alpha)=\lambda^{n} \widehat{h}_{\mathrm{NS}}^{q}(X, \alpha)
$$

for all $\alpha \in \mathrm{NS}_{\mathbb{R}}(X)$ and all $\lambda \geqslant 0$. Notice that $\widehat{h}_{\mathrm{NS}}^{q}(X, \alpha)$ and $\widehat{h}_{\mathrm{NS}}^{\leqslant q}(X, \alpha)$ are always finite thanks to holomorphic Morse inequalities (see below).

\section{(1.5) Proposition.}

(a) For $L \in \operatorname{Pic}_{D}(X)$, one has $\widehat{h}^{q}(X, L)=\widehat{h}^{q}\left(X, c_{1}(L)\right)$ and $\widehat{h}^{\leqslant q}(X, L)=\widehat{h}_{\mathrm{DNS}}^{\leqslant q}\left(X, c_{1}(L)\right)$, in particular asymptotic cohomology depends only on the numerical class of $L$.

(b) The map $\alpha \mapsto \widehat{h}_{\mathrm{DNS}}^{q}(X, \alpha)$ is (locally) Lipschitz continuous on $\operatorname{DNS}_{\mathbb{R}}(X)$.

(c) When $q=0, \widehat{h}_{\mathrm{DNS}}^{0}(X, \alpha)$ and $\widehat{h}_{\mathrm{NS}}^{0}(X, \alpha)$ coincide on $\operatorname{DNS}_{\mathbb{R}}(X)$ and the limsups are limits.

The proof is derived from arguments quite similar to those already developed in [Kür06] (see also [Dem10a] for the non projective situation). If $D=\sum p_{j} D_{j}$ is an 
integral divisor, we define its norm to be $\|D\|=\sum\left|p_{j}\right| \operatorname{Vol}_{\omega}\left(D_{j}\right)$, where the volume of an irreducible divisor is computed by means of a given Hermitian metric $\omega$ on $X$; in other words, this is precisely the mass of the current of integration $[D]$ with respect to $\omega$. Clearly, since $X$ is compact, we get equivalent norms for all choices of Hermitian metrics $\omega$ on $X$. We can also use $\omega$ to fix a normalized metric on $H_{\mathrm{BC}}^{1,1}(X, \mathbb{R})$. Elementary properties of potential theory show that $\left\|c_{1}(\mathscr{G}(D))\right\| \leqslant C\|D\|$ for some constant $C>0$ (but the converse inequality is of course wrong in most cases). Proposition 1.5 is a simple consequence of the more precise cohomology estimates (1.9) which will be obtained below. The special case $q=0$ is easier, in fact, one can get non zero values for $\widehat{h}^{0}(X, L)$ only when $L$ is big, i.e. when $X$ is Moishezon (so that we are always reduced to the divisorial situation); the fact that limsups are limits was proved in II (6.5). We postpone the proof to section 19, which will provide stronger results based on approximate Zariski decomposition.

(1.6) Lemma. Let $X$ be a compact complex $n$-fold. Then for every coherent sheaf $\mathscr{F}$ on $X$, there is a constant $C_{\mathscr{F}}>0$ such that for every holomorphic line bundle $L$ on $X$ we have

$$
h^{q}\left(X, \mathscr{F} \otimes \mathscr{O}_{X}(L)\right) \leqslant C_{\mathscr{F}}\left(\left\|c_{1}(L)\right\|+1\right)^{p}
$$

where $p=\operatorname{dim} \operatorname{Supp} \mathscr{F}$.

Proof. We prove the result by induction on $p$; it is indeed clear for $p=0$ since we then have cohomology only in degree 0 and the dimension of $H^{0}\left(X, \mathscr{F} \otimes \mathscr{\sigma}_{X}(L)\right)$ does not depend on $L$ when $\mathscr{F}$ has finite support. Let us consider the support $Y$ of $\mathscr{F}$ and a resolution of singularity $\mu: \widehat{Y} \rightarrow Y$ of the corresponding (reduced) analytic space. Then $\mathscr{F}$ is an $\mathscr{\sigma}_{Y}$-module for some non necessarily reduced complex structure $\mathscr{\sigma}_{Y}=\mathscr{G}_{X} / \mathcal{F}$ on $Y$. We can look at the reduced structure $\mathscr{G}_{Y \text {,red }}=\mathscr{O}_{X} / \mathscr{F}, \mathscr{F}=\sqrt{\mathscr{T}}$, and filter $\mathscr{F}$ by $\mathscr{G}^{k} \mathscr{F}, k \geqslant 0$. Since $\mathscr{F}^{k} \mathscr{F} / \mathscr{F}^{k+1} \mathscr{F}$ is a coherent $\mathscr{O}_{Y, \text { red }}$-module, we can easily reduce the situation to the case where $Y$ is reduced and $\mathscr{F}$ is an $\mathscr{O}_{Y}$-module. In that case the cohomology

$$
H^{q}\left(X, \mathscr{F} \otimes \mathscr{O}_{X}(L)\right)=H^{q}\left(Y, \mathscr{F} \otimes \mathscr{O}_{Y}\left(L_{\mid Y}\right)\right)
$$

just lives on the reduced space $Y$.

Now, we have an injective sheaf morphism $\mathscr{F} \rightarrow \mu_{*} \mu^{*} \mathscr{F}$ whose cokernel $\mathscr{G}$ has support in dimension $<p$. By induction on $p$, we conclude from the exact sequence that

$$
\left|h^{q}\left(X, \mathscr{F} \otimes \mathscr{O}_{X}(L)\right)-h^{q}\left(X, \mu_{*} \mu^{*} \mathscr{F} \otimes \mathscr{O}_{X}(L)\right)\right| \leqslant C_{1}\left(\left\|c_{1}(L)\right\|+1\right)^{p-1} .
$$

The functorial morphisms

$$
\begin{aligned}
& \mu^{*}: H^{q}\left(Y, \mathscr{F} \otimes \mathscr{G}_{Y}\left(L_{\mid Y}\right)\right) \rightarrow H^{q}\left(\widehat{Y}, \mu^{*} \mathscr{F} \otimes \mathscr{G}_{\widehat{Y}}\left(\mu^{*} L\right)_{\mid Y}\right), \\
& \mu_{*}: H^{q}\left(\widehat{Y}, \mu^{*} \mathscr{F} \otimes \mathscr{G}_{\widehat{Y}}\left(\mu^{*} L\right)_{\mid Y}\right) \rightarrow H^{q}\left(Y, \mu_{*} \mu^{*} \mathscr{F} \otimes \mathscr{G}_{Y}\left(L_{\mid Y}\right)\right)
\end{aligned}
$$

yield a composition

$$
\mu_{*} \circ \mu^{*}: H^{q}\left(Y, \mathscr{F} \otimes \mathscr{\sigma}_{Y}\left(L_{\mid Y}\right)\right) \rightarrow H^{q}\left(Y, \mu_{*} \mu^{*} \mathscr{F} \otimes \mathscr{O}_{Y}\left(L_{\mid Y}\right)\right)
$$

induced by the natural injection $\mathscr{F} \rightarrow \mu_{*} \mu^{*} \mathscr{F}$. This implies

$$
h^{q}\left(Y, \mathscr{F} \otimes \mathscr{O}_{Y}\left(L_{\mid Y}\right)\right) \leqslant h^{q}\left(\widehat{Y}, \mu^{*} \mathscr{F} \otimes \mathscr{O}_{\widehat{Y}}\left(\mu^{*} L_{\mid Y}\right)\right)+C_{1}\left(\left\|c_{1}(L)\right\|+1\right)^{p-1} .
$$


By taking a suitable modification $\mu^{\prime}: Y^{\prime} \rightarrow Y$ of the desingularization $\widehat{Y}$, we can assume that $\left(\mu^{\prime}\right)^{*} \mathscr{F}$ is locally free modulo torsion. Then we are reduced to the case where $\mathscr{F}^{\prime}=$ $\left(\mu^{\prime}\right)^{*} \mathscr{F}$ is a locally free sheaf on a smooth manifold $Y^{\prime}$, and $L^{\prime}=\left(\mu^{\prime}\right)^{*} L_{\mid Y}$. In this case, we apply Morse inequalities to conclude that $h^{q}\left(Y^{\prime}, \mathscr{F}^{\prime} \otimes \mathscr{O}_{Y^{\prime}}\left(L^{\prime}\right)\right) \leqslant C_{2}\left(\left\|c_{1}\left(L^{\prime}\right)\right\|+1\right)^{p}$. Since $\left\|c_{1}\left(L^{\prime}\right)\right\| \leqslant C_{3}\left\|c_{1}(L)\right\|$ by pulling-back, the statement follows easily.

(1.7) Corollary. For every irreducible divisor $D$ on $X$, there exists a constant $C_{D}$ such that

$$
h^{q}\left(D, \mathscr{\sigma}_{D}\left(L_{\mid D}\right)\right) \leqslant C_{D}\left(\left\|c_{1}(L)\right\|+1\right)^{n-1}
$$

Proof. It is enough to apply Lemma 1.6 with $\mathscr{F}=\left(i_{D}\right)_{*}{ }^{\sigma_{D}}$ where $i_{D}: D \rightarrow X$ is the injection.

(1.8) Remark. It is very likely that one can get an "elementary" proof of Lemma 1.6 without invoking resolutions of singularities, e.g. by combining the Cartan-Serre finiteness argument along with the standard Serre-Siegel proof based ultimately on the Schwarz lemma. In this context, one would invoke $L^{2}$ estimates to get explicit bounds for the homotopy operators between Čech complexes relative to two coverings $\boldsymbol{U}=\left(B\left(x_{j}, r_{j}\right)\right)$, $\mathcal{U}^{\prime}=\left(B\left(x_{j}, r_{j} / 2\right)\right)$ of $X$ by concentric balls. By exercising enough care in the estimates, it is likely that one could reach an explicit dependence $C_{D} \leqslant C^{\prime}\|D\|$ for the constant $C_{D}$ of Corollary 1.7. The proof would of course become much more technical than the rather naive brute force approach we have used.

(1.9) Theorem. Let $X$ be a compact complex manifold. Fix a finitely generated subgroup $\Gamma$ of the group of $\mathbb{Z}$-divisors on $X$. Then there are constants $C, C^{\prime}$ depending only on $X$, its Hermitian metric $\omega$ and the subgroup $\Gamma$, satisfying the following properties.

(a) Let $L$ and $L^{\prime}=L \otimes \mathscr{O}(D)$ be holomorphic line bundles on $X$, where $D \in \Gamma$ is an integral divisor. Then

$$
\left|h^{q}\left(X, L^{\prime}\right)-h^{q}(X, L)\right| \leqslant C\left(\left\|c_{1}(L)\right\|+\|D\|\right)^{n-1}\|D\| \text {. }
$$

(b) On the subspace $\operatorname{DNS}_{\mathbb{R}}(X)$, the asymptotic q-cohomology function $\widehat{h}_{\mathrm{DNS}}^{q}$ satisfies a global estimate

$$
\left|\widehat{h}_{\mathrm{DNS}}^{q}(X, \beta)-\widehat{h}_{\mathrm{DNS}}^{q}(X, \alpha)\right| \leqslant C^{\prime}(\|\alpha\|+\|\beta\|)^{n-1}\|\beta-\alpha\| \text {. }
$$

In particular (without any further assumption on $X), \widehat{h}_{\mathrm{DNS}}^{q}$ is locally Lipschitz continuous on $\operatorname{DNS}_{\mathbb{R}}(X)$.

Proof. (a) We want to compare the cohomology of $L$ and $L^{\prime}=L \otimes \mathscr{O}(D)$ on $X$. For this we write $D=D_{+}-D_{-}$, and compare the cohomology of the pairs $L$ and $L_{1}=$ $L \otimes \mathscr{O}\left(-D_{-}\right)$one one hand, and of $L^{\prime}$ and $L_{1}=L^{\prime} \otimes \mathscr{O}\left(-D_{+}\right)$on the other hand. Since $\left\|c_{1}(\mathscr{G}(D))\right\| \leqslant C\|D\|$ by elementary potential theory, we see that is is enough to consider the case of a negative divisor, i.e. $L^{\prime}=L \otimes \sigma(-D), D \geqslant 0$. If $D$ is an irreducible divisor, we use the exact sequence

$$
0 \rightarrow L \otimes \mathscr{O}(-D) \rightarrow L \rightarrow \mathscr{O}_{D} \otimes L_{\mid D} \rightarrow 0
$$


and conclude by Corollary 1.7 that

$$
\begin{aligned}
& \left|h^{q}(X, L \otimes \mathscr{G}(-D))-h^{q}(X, L)\right| \leqslant h^{q}\left(D, \mathscr{O}_{D} \otimes L_{\mid D}\right)+h^{q-1}\left(D, \mathscr{O}_{D} \otimes L_{\mid D}\right) \\
& \leqslant 2 C_{D}\left(\left\|c_{1}(L)\right\|+1\right)^{n-1} \text {. }
\end{aligned}
$$

For $D=\sum p_{j} D_{j} \geqslant 0$, we easily get by induction

$$
\left|h^{q}(X, L \otimes \Theta(-D))-h^{q}(X, L)\right| \leqslant 2 \sum_{j} p_{j} C_{D_{j}}\left(\left\|c_{1}(L)\right\|+\sum_{k} p_{k}\left\|\nabla_{k}\right\|+1\right)^{n-1} .
$$

If we knew that $C_{D} \leqslant C^{\prime}\|D\|$ as expected in Remark 1.6, then the argument would be complete without any restriction on $D$. The trouble disappears if we fix $D$ in a finitely generated subgroup $\Gamma$ of divisors, because only finitely many irreducible components appear in that case, and so we have to deal with only finitely many constants $C_{D_{j}}$. Property 1.9 (a) is proved.

(b) Fix once for all a finite set of divisors $\left(\Delta_{j}\right)_{1 \leqslant j \leqslant t}$ providing a basis of $\operatorname{DNS}_{\mathbb{R}}(X) \subset$ $H_{\mathrm{BC}}^{1,1}(X, \mathbb{R})$. Take two elements $\alpha$ and $\beta$ in $\operatorname{DNS}_{\mathbb{R}}(X)$, and fix $\varepsilon>0$. Then $\beta-\alpha$ can be $\varepsilon$-approximated by a $\mathbb{Q}$-divisor $\sum \lambda_{j} D_{j}, \lambda_{j} \in \mathbb{Q}$, and we can find a pair $(k, L)$ with $k$ arbitrary large such that $\frac{1}{k} c_{1}(L)$ is $\varepsilon$-close to $\alpha$ and $n ! / k^{n} h^{q}(X, L)$ approaches $\widehat{h}_{\mathrm{DNS}}^{q}(X, \alpha)$ by $\varepsilon$. Then $\frac{1}{k} L+\sum \lambda_{j} \Delta_{j}$ approaches $\beta$ as closely as we want. When approximating $\beta-\alpha$, we can arrange that $k \lambda_{j}$ is an integer by taking $k$ large enough. Then $\beta$ is approximated by $\frac{1}{k} c_{1}\left(L^{\prime}\right)$ with $L^{\prime}=L \otimes \mathscr{O}\left(\sum k \lambda_{j} \Delta_{j}\right)$. Property (a) implies

$$
\begin{aligned}
h^{q}\left(X, L^{\prime}\right)-h^{q}(X, L) & \geqslant-C\left(\left\|c_{1}(L)\right\|+\left\|\sum k \lambda_{j} \Delta_{j}\right\|\right)^{n-1}\left\|\sum k \lambda_{j} \Delta_{j}\right\| \\
& \geqslant-C k^{n}(\|\alpha\|+\varepsilon+\|\beta-\alpha\|+\varepsilon)^{n-1}(\|\beta-\alpha\|+\varepsilon) .
\end{aligned}
$$

We multiply the previous inequality by $n ! / k^{n}$ and get in this way

$$
\frac{n !}{k^{n}} h^{q}\left(X, L^{\prime}\right) \geqslant \widehat{h}_{\mathrm{DNS}}^{q}(X, \alpha)-\varepsilon-C^{\prime}(\|\alpha\|+\|\beta\|+\varepsilon)^{n-1}(\|\beta-\alpha\|+\varepsilon) .
$$

By taking the limsup and letting $\varepsilon \rightarrow 0$, we finally obtain

$$
\widehat{h}_{\mathrm{DNS}}^{q}(X, \beta)-\widehat{h}_{\mathrm{DNS}}^{q}(X, \alpha) \geqslant-C^{\prime}(\|\alpha\|+\|\beta\|)^{n-1}\|\beta-\alpha\| .
$$

Property 1.9 (b) follows by exchanging the roles of $\alpha$ and $\beta$.

\section{Transcendental asymptotic cohomology functions}

Our ambition is to extend the function $\widehat{h}_{\mathrm{NS}}^{q}$ in a natural way to the full cohomology group $H_{\mathrm{BC}}^{1,1}(X, \mathbb{R})$. The main trouble, already when $X$ is projective algebraic, is that the Picard number $\rho(X)=\operatorname{dim}_{\mathbb{R}} \mathrm{NS}_{\mathbb{R}}(X)$ may be much smaller than $\operatorname{dim}_{\mathbb{R}} H_{\mathrm{BC}}^{1,1}(X, \mathbb{R})$, namely, there can be rather few integral classes of type $(1,1)$ on $X$. It is well known for instance that $\rho(X)=0$ for a generic complex torus of dimension $n \geqslant 2$, while $\operatorname{dim}_{\mathbb{R}} H_{\mathrm{BC}}^{1,1}(X, \mathbb{R})=n^{2}$. However, if we look at the natural morphism

$$
H_{\mathrm{BC}}^{1,1}(X, \mathbb{R}) \rightarrow H_{\mathrm{DR}}^{2}(X, \mathbb{R}) \simeq H^{2}(X, \mathbb{R})
$$


to de Rham cohomology, then $H^{2}(X, \mathbb{Q})$ is dense in $H^{2}(X, \mathbb{R})$. Therefore, given a class $\alpha \in H_{\mathrm{BC}}^{1,1}(X, \mathbb{R})$ and a smooth $d$-closed $(1,1)$-form $u$ in $\alpha$, we can find an infinite sequence $\frac{1}{k} L_{k}(k \in S \subset \mathbb{N})$ of topological $\mathbb{Q}$-line bundles, equipped with Hermitian metrics $h_{k}$ and compatible connections $\nabla_{k}$ such that the curvature forms $\frac{1}{k} \Theta_{\nabla_{k}}$ converge to $u$. By using Kronecker's approximation with respect to the integral lattice $H^{2}(X, \mathbb{Z}) /$ torsion $\subset$ $H^{2}(X, \mathbb{R})$, we can even achieve a fast diophantine approximation

$$
\left\|\Theta_{\nabla_{k}}-k u\right\| \leqslant C k^{-1 / b_{2}}
$$

for a suitable infinite subset $k \in S \subset \mathbb{N}$ of multipliers. Then in particular

$$
\left\|\Theta_{\nabla_{k}}^{0,2}\right\|=\left\|\Theta_{\nabla_{k}}^{0,2}-k u^{0,2}\right\| \leqslant C k^{-1 / b_{2}}
$$

and we see that $\left(L_{k}, h_{k}, \nabla_{k}\right)$ is a $C^{\infty}$ Hermitian line bundle which is extremely close to being holomorphic, since $\left(\nabla_{k}^{0,1}\right)^{2}=\Theta_{\nabla_{k}}^{0,2}$ is very small. We fix a Hermitian metric $\omega$ on $X$ and introduce the complex Laplace-Beltrami operator

$$
\bar{\square}_{k, q}=\left(\nabla_{k}^{0,1}\right)\left(\nabla_{k}^{0,1}\right)^{*}+\left(\nabla_{k}^{0,1}\right)^{*}\left(\nabla_{k}^{0,1}\right) \quad \text { acting on } L^{2}\left(X, \Lambda^{0, q} T_{X}^{*} \otimes L_{k}\right) \text {. }
$$

We look at its eigenspaces with respect to the $L^{2}$ metric induced by $\omega$ on $X$ and $h_{k}$ on $L_{k}$. In the holomorphic case, Hodge theory tells us that the 0-eigenspace is isomorphic to $H^{q}\left(X, \odot\left(L_{k}\right)\right)$, but in the "almost holomorphic case" the 0-eigenvalues deviate from 0 , essentially by a shift of the order of magnitude of $\left\|\Theta_{\nabla_{k}}^{0,2}\right\| \sim k^{-1 / b_{2}}$ (see also the $\mathrm{PhD}$ thesis of L. Laeng [Lae02], Chapter 4, for more details). It is thus natural to introduce in this case

(2.3) Definition. Let $X$ be a compact complex manifold and $\alpha \in H_{\mathrm{BC}}^{1,1}(X, \mathbb{R})$ an arbitrary Bott-Chern $(1,1)$-class. We define the "transcendental" asymptotic q-cohomology functions to be

$$
\begin{array}{ccc}
\widehat{h}_{\mathrm{tr}}^{q}(X, \alpha)=\inf _{u \in \alpha} & \limsup _{\substack{\varepsilon \rightarrow 0, k \rightarrow+\infty, L_{k}, h_{k}, \nabla_{k}, \frac{1}{k} \Theta_{\nabla_{k}} \rightarrow u \\
k^{n}}} & \frac{n !}{k^{n}} N\left(\bar{\square}_{k, q}, \leqslant \varepsilon\right) \\
\widehat{h}_{\mathrm{tr}}^{\leqslant q}(X, \alpha)=\inf _{u \in \alpha} & \limsup _{\substack{\varepsilon \rightarrow 0, k \rightarrow+\infty, L_{k}, h_{k}, \nabla_{k}, \frac{1}{k} \Theta_{\nabla_{k}} \rightarrow u \\
k^{n}}} \frac{n !}{\sum_{0 \leqslant j \leqslant q}(-1)^{q-j} N\left(\bar{\square}_{k, j}, \leqslant k \varepsilon\right)}
\end{array}
$$

where the limsup runs over all 5 -tuples $\left(\varepsilon, k, L_{k}, h_{k}, \nabla_{k}\right)$, and where $N\left(\bar{\square}_{k, q}, k \varepsilon\right)$ denotes the sum of dimensions of all eigenspaces of eigenvalues at most equal to $k \varepsilon$ for the LaplaceBeltrami operator $\bar{\square}_{k, q}$ on $L^{2}\left(X, \Lambda^{0, q} T_{X}^{*} \otimes L_{k}\right)$ associated with $\left(L_{k}, h_{k}, \nabla_{k}\right)$ and the base Hermitian metric $\omega$.

The word "transcendental" refers here to the fact that we deal with classes $\alpha$ of type $(1,1)$ which are not algebraic or even analytic. Of course, in the definition, we could have restricted the limsup to families satisfying a better approximation property $\left\|\frac{1}{k} \Theta_{\nabla_{k}}-u\right\| \leqslant$ $C k^{-1-1 / b_{2}}$ for some large constant $C$ (this would lead a priori to a smaller limsup, but there is enough stability in the parameter dependence of the spectrum for making such a change irrelevant). The minimax principle easily shows that Definition 2.1 does not depend on $\omega$, as the eigenvalues are at most multiplied or divided by constants under a 
change of base metric. When $\alpha \in \mathrm{NS}_{\mathbb{R}}(X)$, by restricting our families $\left\{\left(\varepsilon, k, L_{k}, h_{k}, \nabla_{k}\right)\right\}$ to the case of holomorphic line bundles only, we get the obvious inequalities

$$
\begin{array}{ll}
\widehat{h}_{\mathrm{NS}}^{q}(X, \alpha) \leqslant \widehat{h}_{\mathrm{tr}}^{q}(X, \alpha), & \forall \alpha \in \mathrm{NS}_{\mathbb{R}}(X), \\
\widehat{h}_{\mathrm{NS}}^{\leqslant q}(X, \alpha) \leqslant \widehat{h}_{\mathrm{tr}}^{\leqslant q}(X, \alpha), \quad \forall \alpha \in \mathrm{NS}_{\mathbb{R}}(X) .
\end{array}
$$

It is natural to raise the question whether these inequalities are always equalities. Hopefully, the calculation of the quantities $\lim _{k \rightarrow+\infty} \frac{n !}{k^{n}} N\left(\bar{\square}_{k, q}, \leqslant k \varepsilon\right)$ is a problem of spectral theory which is completely understood thanks to Chapter I (see also [Dem85, 91]). In fact, by Corollary I (1.13), the above limit can be evaluated explicitly for any value of $\varepsilon \in \mathbb{R}$, except possibly for a countable number of values of $\varepsilon$ for which jumps occur; one only has to take care that the non-integrability of $\bar{\partial}$ due to the diophantine approximation does not contribute asymptotically to the eigenvalue distribution, a fact which follows immediately from (2.2) (cf. [Lae02]).

(2.5) Theorem. With the above notations and assumptions, let us introduce at each point $x$ in $X$ the "spectral density function", defined as a finite sum

$$
\nu_{u}(\lambda)=\frac{n !(4 \pi)^{s-n}}{(n-s) !}\left|u_{1}\right| \ldots\left|u_{s}\right| \sum_{\left(p_{1}, \ldots, p_{s}\right) \in \mathbb{N}^{s}}\left(\lambda-\sum_{j=1}^{s}\left(2 p_{j}+1\right)\left|u_{j}\right|\right)_{+}^{n-s}
$$

where $s=s(x)$ is the rank of the real $(1,1)$-form $u$ at $x$, and $u_{j}, 1 \leqslant j \leqslant s$, its non zero eigenvalues with respect to the base Hermitian metric $\omega$, and $u_{s+1}=\ldots=u_{n}=0$. For each multi-index $J \subset\{1,2, \ldots, n\}$, let us set $u_{J}=\sum_{j \in J} u_{j}$. Then the asymptotic spectrum of $\bar{\square}_{k, q}$ admits the estimate

$$
\lim _{k \rightarrow+\infty} \frac{n !}{k^{n}} N\left(\bar{\square}_{k, q}, \leqslant k \lambda\right)=\int_{X} \sum_{|J|=q} \nu_{u}\left(\lambda+u_{\mathrm{C} J}-u_{J}\right) d V_{\omega}
$$

except possibly for a countable number of values of $\lambda$ which are discontinuities of the right hand integral as an increasing integral of $\lambda$.

(2.6) Corollary. We have (as a limit rather than just a lim sup) the spectral estimate

$$
\lim _{\varepsilon \rightarrow 0, k \rightarrow+\infty, L_{k}, h_{k}, \nabla_{k}, \frac{1}{k} \Theta_{\nabla_{k}} \rightarrow u} \frac{n !}{k^{n}} N\left(\bar{\square}_{k, q}, \leqslant k \varepsilon\right)=\int_{X(u, q)}(-1)^{q} u^{n} .
$$

Coming back to the transcendental asymptotic cohomology functions, we get the following fundamental result, which gives in some sense an explicit formula for $\widehat{h}_{\mathrm{tr}}^{q}(X, \alpha)$ and $\widehat{h}_{\mathrm{tr}}^{\leqslant q}(X, \alpha)$ in terms of Monge-Ampère operators.

(2.7) Theorem. The limsup's defining $\widehat{h}_{\mathrm{tr}}^{q}(X, \alpha)$ and $\widehat{h}_{\mathrm{tr}}^{\leqslant q}(X, \alpha)$ are limits, and we have

$$
\begin{array}{ll}
\widehat{h}_{\mathrm{tr}}^{q}(X, \alpha) & =\inf _{u \in \alpha} \int_{X(u, q)}(-1)^{q} u^{n} \quad \text { (u smooth). } \\
\text { (b) } \widehat{h}_{\mathrm{tr}}^{\leqslant q}(X, \alpha) & \left.=\inf _{u \in \alpha} \int_{X(u, \leqslant q)}(-1)^{q} u^{n} \quad \text { (u smooth }\right) .
\end{array}
$$


Now, if $L \rightarrow X$ is a holomorphic line bundle, we have by definition

$$
\widehat{h}^{\leqslant q}(X, L) \leqslant \widehat{h}_{\mathrm{DNS}}^{\leqslant q}\left(X, c_{1}(L)\right) \leqslant \widehat{h}_{\mathrm{NS}}^{\leqslant q}\left(X, c_{1}(L)\right) \leqslant \inf _{u \in c_{1}(L)} \int_{X(u, \leqslant q)}(-1)^{q} u^{n}
$$

( $u$ smooth), where the last inequality is a consequence of holomorphic Morse inequalities. We hope for the following conjecture which would imply that we always have equalities.

(2.9) Conjecture. For every holomorphic line bundle $L \rightarrow X$ on a compact complex manifold $X$, we have

$$
\begin{aligned}
& \widehat{h}^{q}(X, L)=\inf _{u \in \alpha} \int_{X(u, q)}(-1)^{q} u^{n}, \quad \text { u smooth, } \\
& \widehat{h}^{\leqslant q}(X, L)=\inf _{u \in \alpha} \int_{X(u, \leqslant q)}(-1)^{q} u^{n}, \quad \text { u smooth. }
\end{aligned}
$$

Since the right hand side is easily seen to depend continuously on $\alpha \in H_{\mathrm{BC}}^{1,1}(X, \mathbb{C})$, one would get :

(2.10) Corollary of the conjecture. If (2.9) holds true, then

$$
\widehat{h}_{\mathrm{NS}}^{q}(X, \alpha)=\widehat{h}_{\mathrm{tr}}^{q}(X, \alpha) \quad \text { and }
$$

(b) $\quad \widehat{h}_{\mathrm{NS}}^{\leqslant q}(X, \alpha)=\widehat{h}_{\mathrm{tr}}^{\leqslant q}(X, \alpha)$

for all classes $\alpha \in \mathrm{NS}_{\mathbb{R}}(X)$.

In general, equalities $2.9(\mathrm{a}, \mathrm{b})$ seem rather hard to prove. In some sense, they would stand as an asymptotic converse of the Andreotti-Grauert theorem [AG62] : under a suitable $q$-convexity assumption, the latter asserts the vanishing of related cohomology groups in degree $q$; here, conversely, assuming a known growth of these groups in degree $q$, we expect to be able to say something about the $q$-index sets of suitable Hermitian metrics on the line bundles under consideration. The only cases where we have a positive answer to Question 2.8 are when $X$ is projective and $q=0$ or $\operatorname{dim} X \leqslant 2$ (see Theorems 4.1 and 5.1 below). In the general setting of compact complex manifolds, we also hope for the following "transcendental" case of holomorphic Morse inequalities.

(2.11) Conjecture. Let $X$ be a compact complex $n$-fold and $\alpha$ an arbitrary cohomology class in $H_{\mathrm{BC}}^{1,1}(X, \mathbb{R})$. Then the volume, defined as the supremum

$$
\operatorname{Vol}(\alpha):=\sup _{0<T \in \alpha} \int_{X \backslash \operatorname{Sing}(T)} T^{n}
$$

extended to all Kähler currents $T \in \alpha$ with analytic singularities (see Definition II (4.4)), satisfies

$$
\operatorname{Vol}(\alpha) \geqslant \sup _{u \in \alpha} \int_{X(u, 0) \cup X(u, 1)} u^{n}
$$

where $u$ runs over all smooth closed $(1,1)$ forms. In particular, if the right hand side is positive, then a contains a Kähler current. 
By the holomorphic Morse inequalities, Conjecture 2.11 holds true in case $\alpha$ is an integral class. Our hope is that the general case can be attained by the diophantine approximation technique described earlier; there are however major hurdles, see [Lae02] for a few hints on these issues.

\section{Invariance by modification}

We end this section by the observation that the asymptotic cohomology functions are invariant by modification, namely that for every modification $\mu: \widetilde{X} \rightarrow X$ and every line bundle $L$ we have e.g.

$$
\widehat{h}^{q}(X, L)=\widehat{h}^{q}\left(\widetilde{X}, \mu^{*} L\right) .
$$

In fact the Leray spectral sequence provides an $E_{2}$ term

$$
E_{2}^{p, q}=H^{p}\left(X, R^{q} \mu_{*} \mathscr{\sigma}_{\widetilde{X}}\left(\mu^{*} L^{\otimes k}\right)\right)=H^{p}\left(X, \mathscr{\sigma}_{X}\left(L^{\otimes k}\right) \otimes R^{q} \mu_{*} \mathscr{G}_{\widetilde{X}}\right) .
$$

Since $R^{q} \mu_{*} \mathscr{G}_{\widetilde{X}}$ is equal to $\mathscr{G}_{X}$ for $q=0$ and is supported on a proper analytic subset of $X$ for $q \geqslant 1$, one infers that $h^{p}\left(X, \mathscr{\sigma}_{X}\left(L^{\otimes k} \otimes R^{q} \mu_{*} \mathscr{G}_{\widetilde{X}}\right)=O\left(k^{n-1}\right)\right.$ for all $q \geqslant 1$. The spectral sequence implies that

$$
h^{q}\left(X, L^{\otimes k}\right)-\widehat{h}^{q}\left(\widetilde{X}, \mu^{*} L^{\otimes k}\right)=O\left(k^{n-1}\right) .
$$

We claim that the Morse integral infimums are also invariant by modification.

(3.2) Proposition. Let $(X, \omega)$ be a compact Kähler manifold, $\alpha \in H^{1,1}(X, \mathbb{R})$ a real cohomology class and $\mu: \widetilde{X} \rightarrow X$ a modification. Then

$$
\begin{aligned}
& \inf _{u \in \alpha} \int_{X(u, q)}(-1)^{q} u^{n}=\inf _{v \in \mu^{*} \alpha} \int_{X(v, q)}(-1)^{q} v^{n}, \\
& \inf _{u \in \alpha} \int_{X(u, \leqslant q)}(-1)^{q} u^{n}=\inf _{v \in \mu^{*} \alpha} \int_{X(v, \leqslant q)}(-1)^{q} v^{n} .
\end{aligned}
$$

Proof. Given $u \in \alpha$ on $X$, we obtain Morse integrals with the same values by taking $v=\mu^{*} u$ on $\widetilde{X}$, hence the infimum on $\widetilde{X}$ is smaller or equal to what is on $X$. Conversely, we have to show that given a smooth representative $v \in \mu^{*} \alpha$ on $\tilde{X}$, one can find a smooth representative $u \in X$ such that the Morse integrals do not differ much. We can always assume that $\widetilde{X}$ itself is Kähler, since by Hironaka [Hir64] any modification $\widetilde{X}$ is dominated by a composition of blow-ups of $X$. Let us fix some $u_{0} \in \alpha$ and write

$$
v=\mu^{*} u_{0}+d d^{c} \varphi
$$

where $\varphi$ is a smooth function on $\widetilde{X}$. We adjust $\varphi$ by a constant in such a way that $\varphi \geqslant 1$ on $\widetilde{X}$. There exists an analytic set $S \subset X$ such that $\mu: \widetilde{X} \backslash \mu^{-1}(S) \rightarrow X \backslash S$ is a biholomorphism, and a quasi-psh function $\psi_{S}$ which is smooth on $X \backslash S$ and has $-\infty$ logarithmic poles on $S$ (see e.g. [Dem82]). We define

$$
\widetilde{u}=\mu^{*} u_{0}+d d^{c} \max _{\varepsilon_{0}}\left(\varphi+\delta \psi_{S} \circ \mu, \quad 0\right)=v+d d^{c} \max _{\varepsilon_{0}}\left(\delta \psi_{S} \circ \mu,-\varphi\right)
$$


where $\max _{\varepsilon_{0}}, 0<\varepsilon_{0}<1$, is a regularized max function and $\delta>0$ is very small. By construction $\widetilde{u}$ coincides with $\mu^{*} u_{0}$ in a neighborhood of $\mu^{-1}(S)$ and therefore $\widetilde{u}$ descends to a smooth closed $(1,1)$-form $u$ on $X$ which coincides with $u_{0}$ near $S$, so that $\widetilde{u}=\mu^{*} u$. Clearly $\widetilde{u}$ converges uniformly to $v$ on every compact subset of $\widetilde{X} \backslash \mu^{-1}(S)$ as $\delta \rightarrow 0$, so we only have to show that the Morse integrals are small (uniformly in $\delta$ ) when restricted to a suitable small neighborhood of the exceptional set $E=\mu^{-1}(S)$. Take a sufficiently large Kähler metric $\widetilde{\omega}$ on $\widetilde{X}$ such that

$$
-\frac{1}{2} \widetilde{\omega} \leqslant v \leqslant \frac{1}{2} \widetilde{\omega}, \quad-\frac{1}{2} \widetilde{\omega} \leqslant d d^{c} \varphi \leqslant \frac{1}{2} \widetilde{\omega}, \quad-\widetilde{\omega} \leqslant d d^{c} \psi_{S} \circ \mu .
$$

Then $\widetilde{u} \geqslant-\widetilde{\omega}$ and $\widetilde{u} \leqslant \widetilde{\omega}+\delta d d^{c} \psi_{S} \circ \mu$ everywhere on $\widetilde{X}$. As a consequence

$$
\left|\widetilde{u}^{n}\right| \leqslant\left(\widetilde{\omega}+\delta\left(\widetilde{\omega}+d d^{c} \psi_{S} \circ \mu\right)\right)^{n} \leqslant \widetilde{\omega}^{n}+n \delta\left(\widetilde{\omega}+d d^{c} \psi_{S} \circ \mu\right) \wedge\left(\widetilde{\omega}+\delta\left(\widetilde{\omega}+d d^{c} \psi_{S} \circ \mu\right)\right)^{n-1}
$$

thanks to the inequality $(a+b)^{n} \leqslant a^{n}+n b(a+b)^{n-1}$. For any neighborhood $V$ of $\mu^{-1}(S)$ this implies

$$
\int_{V}\left|\widetilde{u}^{n}\right| \leqslant \int_{V} \widetilde{\omega}^{n}+n \delta(1+\delta)^{n-1} \int_{\widetilde{X}} \widetilde{\omega}^{n}
$$

by Stokes formula. We thus see that the integrals are small if $V$ and $\delta$ are small. The reader may be concerned that Monge-Ampère integrals were used with an unbounded potential $\psi_{S}$, but in fact, for any given $\delta$, all the above formulas and estimates are still valid when we replace $\psi_{S}$ by $\max _{\varepsilon_{0}}\left(\psi_{S},-(M+2) / \delta\right)$ with $M=\max _{\widetilde{X}} \varphi$, especially formula (3.3) shows that the form $\widetilde{u}$ is unchanged. Therefore our calculations can be handled by using merely smooth potentials.

\section{Proof of the infimum formula for the volume}

We prove here

(4.1) Theorem. Let $L \rightarrow X$ be a holomorphic line bundle on a projective algebraic manifold $X$. Then

$$
\operatorname{Vol}(X, L)=\inf _{u \in c_{1}(L)} \int_{X(u, 0)} u^{n}
$$

It is enough to show the inequality

$$
\inf _{u \in c_{1}(L)} \int_{X(u, 0)} u^{n} \leqslant \operatorname{Vol}(X, L)
$$

and for this, we have to construct metrics approximating the volume. Let us first assume that $L$ is a big line bundle, i.e. that $\operatorname{Vol}(X, L)>0$. We have seen in II (6.4-6.5) (cf. also [Bck02]) that $\operatorname{Vol}(X, L)$ is obtained as the supremum of $\int_{X \backslash \operatorname{Sing}(T)} T^{n}$ for Kähler currents $T=-\frac{i}{2 \pi} \partial \bar{\partial} h$ with analytic singularities in $c_{1}(L)$; this means that locally $h=e^{-\varphi}$ where $\varphi$ is a strictly plurisubharmonic function which has the same singularities as $c \log \sum\left|g_{j}\right|^{2}$ where $c>0$ and the $g_{j}$ are holomorphic functions. By [Dem92], there exists a blow-up 
$\mu: \tilde{X} \rightarrow X$ such that $\mu^{*} T=[E]+\beta$ where $E$ is a normal crossing divisor on $\tilde{X}$ and $\beta \geqslant 0$ smooth. Moreover, by [BDPP04] we have the orthogonality estimate

$$
[E] \cdot \beta^{n-1}=\int_{E} \beta^{n-1} \leqslant C\left(\operatorname{Vol}(X, L)-\beta^{n}\right)^{1 / 2}
$$

while

$$
\beta^{n}=\int_{\widetilde{X}} \beta^{n}=\int_{X \backslash \operatorname{Sing}(T)} T^{n} \quad \text { approaches } \operatorname{Vol}(X, L) .
$$

In other words, $E$ and $\beta$ become "more and more orthogonal" as $\beta^{n}$ approaches the volume (these properties are summarized by saying that $\mu^{*} T=[E]+\beta$ defines an approximate Zariski decomposition of $c_{1}(L)$, cf. also [Fuj94]). By subtracting to $\beta$ a small linear combination of the exceptional divisors and increasing accordingly the coefficients of $E$, we can achieve that the cohomology class $\{\beta\}$ contains a positive definite form $\beta^{\prime}$ on $\widetilde{X}$ (i.e. the fundamental form of a Kähler metric); we refer e.g. to ([DP04], proof of Lemma 3.5) for details. This means that we can replace $T$ by a cohomologous current such that the corresponding form $\beta$ is actually a Kähler metric, and we will assume for simplicity of notation that this situation occurs right away for $T$. Under this assumption, there exists a smooth closed $(1,1)$-form $v$ belonging to the Bott-Chern cohomology class of $[E]$, such that we have identically $(v-\delta \beta) \wedge \beta^{n-1}=0$ where

$$
\delta=\frac{[E] \cdot \beta^{n-1}}{\beta^{n}} \leqslant C^{\prime}\left(\operatorname{Vol}(X, L)-\beta^{n}\right)^{1 / 2}
$$

for some constant $C^{\prime}>0$. In fact, given an arbitrary smooth representative $v_{0} \in\{[E]\}$, the existence of $v=v_{0}+i \partial \bar{\partial} \psi$ amounts to solving a Laplace equation $\Delta \psi=f$ with respect to the Kähler metric $\beta$, and the choice of $\delta$ ensures that we have $\int_{X} f \beta^{n}=0$ and hence that the equation is solvable. Then $\widetilde{u}:=v+\beta$ is a smooth closed $(1,1)$-form in the cohomology class $\mu^{*} c_{1}(L)$, and its eigenvalues with respect to $\beta$ are of the form $1+\lambda_{j}$ where $\lambda_{j}$ are the eigenvalues of $v$. The Laplace equation is equivalent to the identity $\sum \lambda_{j}=n \delta$. Therefore

$$
\sum_{1 \leqslant j \leqslant n} \lambda_{j} \leqslant C^{\prime \prime}\left(\operatorname{Vol}(X, L)-\beta^{n}\right)^{1 / 2}
$$

The inequality between arithmetic means and geometric means implies

$$
\prod_{1 \leqslant j \leqslant n}\left(1+\lambda_{j}\right) \leqslant\left(1+\frac{1}{n} \sum_{1 \leqslant j \leqslant n} \lambda_{j}\right)^{n} \leqslant 1+C_{3}\left(\operatorname{Vol}(X, L)-\beta^{n}\right)^{1 / 2}
$$

whenever all factors $\left(1+\lambda_{j}\right)$ are nonnegative. By 2.2 (i) we get

$$
\begin{aligned}
\inf _{u \in c_{1}(L)} \int_{X(u, 0)} u^{n} & \leqslant \int_{\widetilde{X}(\widetilde{u}, 0)} \widetilde{u}^{n} \\
& \leqslant \int_{\widetilde{X}} \beta^{n}\left(1+C_{3}\left(\operatorname{Vol}(X, L)-\beta^{n}\right)^{1 / 2}\right) \\
& \leqslant \operatorname{Vol}(X, L)+C_{4}\left(\operatorname{Vol}(X, L)-\beta^{n}\right)^{1 / 2}
\end{aligned}
$$


As $\beta^{n}$ approches $\operatorname{Vol}(X, L)$, this implies inequality (4.1).

We still have to treat the case when $L$ is not big, i.e. $\operatorname{Vol}(X, L)=0$. Let $A$ be an ample line bundle and let $t_{0} \geqslant 0$ be the infimum of real numbers such that $L+t A$ is a big $\mathbb{Q}$-line bundle for $t$ rational, $t>t_{0}$. The continuity of the volume function implies that $0<\operatorname{Vol}(X, L+t A) \leqslant \varepsilon$ for $t>t_{0}$ sufficiently close to $t_{0}$. By what we have just proved, there exists a smooth form $u_{t} \in c_{1}(L+t A)$ such that $\int_{X\left(u_{t}, 0\right)} u_{t}^{n} \leqslant 2 \varepsilon$. Take a Kähler metric $\omega \in c_{1}(A)$ and define $u=u_{t}-t \omega$. Then clearly

$$
\int_{X(u, 0)} u^{n} \leqslant \int_{X\left(u_{t}, 0\right)} u_{t}^{n} \leqslant 2 \varepsilon
$$

hence

$$
\inf _{u \in c_{1}(L)} \int_{X(u, 0)} u^{n}=0 .
$$

Inequality (4.1) is now proved in all cases.

\section{Estimate of the first cohomology group on a projective surface}

Our goal here is to show the following result.

(5.1) Theorem. Let $L \rightarrow X$ be a holomorphic line bundle on a complex projective surface. Then both weak and strong inequalities (1.3) (i) and (1.3) (ii) are equalities for $q=0,1,2$, and the limsup's involved in $\widehat{h}^{q}(X, L)$ and $\widehat{h} \leq q(X, L)$ are limits.

We start with a projective non singular variety $X$ of arbitrary dimension $n$, and will later restrict ourselves to the case when $X$ is a surface. The proof again consists of using (approximate) Zariski decomposition, but now we try to compute more explicitly the resulting curvature forms and Morse integrals; this will turn out to be much easier on surfaces.

Assume first that $L$ is a big line bundle on $X$. As in section 3 , we can find an approximate Zariski decomposition, i.e. a blow-up $\mu: \widetilde{X} \rightarrow X$ and a current $T \in c_{1}(L)$ such $\mu^{*} T=[E]+\beta$, where $E$ an effective divisor and $\beta$ a Kähler metric on $\widetilde{X}$ such that

$$
\operatorname{Vol}(X, L)-\eta<\beta^{n}<\operatorname{Vol}(X, L), \quad \eta \ll 1
$$

(On a projective surface, one could even get exact Zariski decomposition, but we want to remain general as long as possible). By blowing-up further, we may assume that $E$ is a normal crossing divisor. We select a Hermitian metric $h$ on $\mathscr{O}(E)$ and take

$$
u_{\varepsilon}=\frac{i}{2 \pi} \partial \bar{\partial} \log \left(\left|\sigma_{E}\right|_{h}^{2}+\varepsilon^{2}\right)+\Theta_{\Theta(E), h}+\beta \quad \in \quad \mu^{*} c_{1}(L)
$$

where $\sigma_{E} \in H^{0}(\widetilde{X}, \mathscr{O}(E))$ is the canonical section and $\Theta_{\mathscr{O}(E), h}$ the Chern curvature form. Clearly, by the Lelong-Poincaré equation, $u_{\varepsilon}$ converges to $[E]+\beta$ in the weak topology as $\varepsilon \rightarrow 0$. Straightforward calculations yield

$$
u_{\varepsilon}=\frac{i}{2 \pi} \frac{\varepsilon^{2} D_{h}^{1,0} \sigma_{E} \wedge \overline{D_{h}^{1,0} \sigma_{E}}}{\left(\varepsilon^{2}+\left|\sigma_{E}\right|^{2}\right)^{2}}+\frac{\varepsilon^{2}}{\varepsilon^{2}+\left|\sigma_{E}\right|^{2}} \Theta_{E, h}+\beta .
$$


The first term converges to $[E]$ in the weak topology, while the second, which is close to $\Theta_{E, h}$ near $E$, converges pointwise everywhere to 0 on $\widetilde{X} \backslash E$. A simple asymptotic analysis shows that

$$
\left(\frac{i}{2 \pi} \frac{\varepsilon^{2} D_{h}^{1,0} \sigma_{E} \wedge \overline{D_{h}^{1,0} \sigma_{E}}}{\left(\varepsilon^{2}+\left|\sigma_{E}\right|^{2}\right)^{2}}+\frac{\varepsilon^{2}}{\varepsilon^{2}+\left|\sigma_{E}\right|^{2}} \Theta_{E, h}\right)^{p} \rightarrow[E] \wedge \Theta_{E, h}^{p-1}
$$

in the weak topology for $p \geqslant 1$, hence

$$
\lim _{\varepsilon \rightarrow 0} u_{\varepsilon}^{n}=\beta^{n}+\sum_{p=1}^{n}\left(\begin{array}{l}
n \\
p
\end{array}\right)[E] \wedge \Theta_{E, h}^{p-1} \wedge \beta^{n-p} .
$$

In arbitrary dimension, the signature of $u_{\varepsilon}$ is hard to evaluate, and it is also non trivial to decide the sign of the limiting measure $\lim u_{\varepsilon}^{n}$. However, when $n=2$, we get the simpler formula

$$
\lim _{\varepsilon \rightarrow 0} u_{\varepsilon}^{2}=\beta^{2}+2[E] \wedge \beta+[E] \wedge \Theta_{E, h} .
$$

In this case, $E$ can be assumed to be an exceptional divisor (otherwise some part of it would be nef and could be removed from the poles of $T$ ). Hence the matrix $\left(E_{j} \cdot E_{k}\right)$ is negative definite and we can find a smooth Hermitian metric $h$ on $\mathscr{O}(E)$ such that $\left(\Theta_{E, h}\right)_{\mid E}<0$, i.e. $\Theta_{E, h}$ has one negative eigenvalue everywhere along $E$.

(5.5) Lemma. One can adjust the metric $h$ of $\mathscr{O}(E)$ in such a way that $\Theta_{E, h}$ is negative definite on a neighborhood of the support $|E|$ of the exceptional divisor, and $\Theta_{E, h}+\beta$ has signature $(1,1)$ there. (We do not care about the signature far away from $|E|$ ).

Proof. At a given point $x_{0} \in X$, let us fix coordinates and a positive quadratic form $q$ on $\mathbb{C}^{2}$. If we put $\psi_{\varepsilon}(z)=\varepsilon \chi(z) \log \left(1+\varepsilon^{-1} q(z)\right)$ with a suitable cut-off function $\chi$, then the Hessian form of $\psi_{\varepsilon}$ is equal to $q$ at $x_{0}$ and decays rapidly to $O(\varepsilon \log \varepsilon)|d z|^{2}$ away from $x_{0}$. In this way, after multiplying $h$ with $e^{ \pm \psi_{\varepsilon}(z)}$, we can replace the curvature $\Theta_{E, h}\left(x_{0}\right)$ with $\Theta_{E, h}\left(x_{0}\right) \pm q$ without substantially modifying the form away from $x_{0}$. This allows to adjust $\Theta_{E, h}$ to be equal to (say) $-\frac{1}{4} \beta\left(x_{0}\right)$ at any singular point $x_{0} \in E_{j} \cap E_{k}$ in the support of $|E|$, while keeping $\Theta_{E, h}$ negative definite along $E$. In order to adjust the curvature at smooth points $x \in|E|$, we replace the metric $h$ with $h^{\prime}(z)=h(z) \exp \left(-c(z)\left|\sigma_{E}(z)\right|^{2}\right)$. Then the curvature form $\Theta_{E, h}$ is replaced by $\Theta_{E, h^{\prime}}(x)=\Theta_{E_{h}}(x)+c(x)\left|d \sigma_{E}\right|^{2}$ at $x \in|E|$ (notice that $d \sigma_{E}(x)=0$ if $x \in \operatorname{Sing}|E|$ ), and we can always select a real function $c$ so that $\Theta_{E, h^{\prime}}$ is negative definite with one negative eigenvalue between $-1 / 2$ and 0 at any point of $|E|$. Then $\Theta_{E, h^{\prime}}+\beta$ has signature $(1,1)$ near $|E|$.

With this choice of the metric, we see that for $\varepsilon>0$ small, the sum

$$
\frac{\varepsilon^{2}}{\varepsilon^{2}+\left|\sigma_{E}\right|^{2}} \Theta_{E, h}+\beta
$$

is of signature $(2,0)$ or $(1,1)$ (or degenerate of signature $(1,0)$ ), the non positive definite points being concentrated in a neighborhood of $E$. In particular the index set $X\left(u_{\varepsilon}, 2\right)$ is empty, and also

$$
u_{\varepsilon} \leqslant \frac{i}{2 \pi} \frac{\varepsilon^{2} D_{h}^{1,0} \sigma_{E} \wedge \overline{D_{h}^{1,0} \sigma_{E}}}{\left(\varepsilon^{2}+\left|\sigma_{E}\right|^{2}\right)^{2}}+\beta
$$


on a neighborhood $V$ of $|E|$, while $u_{\varepsilon}$ converges uniformly to $\beta$ on $\widetilde{X} \backslash V$. This implies that

$$
\beta^{2} \leqslant \liminf _{\varepsilon \rightarrow 0} \int_{X\left(u_{\varepsilon}, 0\right)} u_{\varepsilon}^{2} \leqslant \limsup _{\varepsilon \rightarrow 0} \int_{X\left(u_{\varepsilon}, 0\right)} u_{\varepsilon}^{2} \leqslant \beta^{2}+2 \beta \cdot E .
$$

Since $\int_{\widetilde{X}} u_{\varepsilon}^{2}=L^{2}=\beta^{2}+2 \beta \cdot E+E^{2}$ we conclude by taking the difference that

$$
-E^{2}-2 \beta \cdot E \leqslant \liminf _{\varepsilon \rightarrow 0} \int_{X\left(u_{\varepsilon}, 1\right)}-u_{\varepsilon}^{2} \leqslant \limsup _{\varepsilon \rightarrow 0} \int_{X\left(u_{\varepsilon}, 1\right)}-u_{\varepsilon}^{2} \leqslant-E^{2} .
$$

Let us recall that $\beta \cdot E \leqslant C\left(\operatorname{Vol}(X, L)-\beta^{2}\right)^{1 / 2}=0\left(\eta^{1 / 2}\right)$ is small by (5.3) and the orthogonality estimate. The asymptotic cohomology is given here by $\widehat{h}^{2}(X, L)=0$ since $h^{2}\left(X, L^{\otimes k}\right)=H^{0}\left(X, K_{X} \otimes L^{\otimes-k}\right)=0$ for $k \geqslant k_{0}$, and we have by Riemann-Roch

$$
\widehat{h}^{1}(X, L)=\widehat{h}^{0}(X, L)-L^{2}=\operatorname{Vol}(X, L)-L^{2}=-E^{2}-\beta \cdot E+O(\eta) .
$$

Here we use the fact that $\frac{n !}{k^{n}} h^{0}\left(X, L^{\otimes k}\right)$ converges to the volume when $L$ is big. All this shows that equality occurs in the Morse inequalities (1.3) when we pass to the infimum. By taking limits in the Neron-Severi space $\mathrm{NS}_{\mathbb{R}}(X) \subset H^{1,1}(X, \mathbb{R})$, we further see that equality occurs as soon as $L$ is pseudo-effective, and the same is true if $-L$ is pseudoeffective by Serre duality.

It remains to treat the case when neither $L$ nor $-L$ are pseudo-effective. Then $\widehat{h}^{0}(X, L)=\widehat{h}^{2}(X, L)=0$, and asymptotic cohomology appears only in degree 1 , with $\widehat{h}^{1}(X, L)=-L^{2}$ by Riemann-Roch. Fix an ample line bundle $A$ and let $t_{0}>0$ be the infimum of real numbers such that $L+t A$ is big for $t$ rational, $t>t_{0}$, resp. let $t_{0}^{\prime}>0$ be the infimum of real numbers $t^{\prime}$ such that $-L+t^{\prime} A$ is big for $t^{\prime}>t_{0}^{\prime}$. Then for $t>t_{0}$ and $t^{\prime}>t_{0}^{\prime}$, we can find a modification $\mu: \widetilde{X} \rightarrow X$ and currents $T \in c_{1}(L+t A)$, $T^{\prime} \in c_{1}\left(-L+t^{\prime} A\right)$ such that

$$
\mu^{*} T=[E]+\beta, \quad \mu^{*} T^{\prime}=[F]+\gamma
$$

where $\beta, \gamma$ are Kähler forms and $E, F$ normal crossing divisors. By taking a suitable linear combination $t^{\prime}(L+t A)-t\left(-L+t^{\prime} A\right)$ the ample divisor $A$ disappears, and we get

$$
\frac{1}{t+t^{\prime}}\left(t^{\prime}[E]+t^{\prime} \beta-t[F]-t \gamma\right) \in \mu^{*} c_{1}(L)
$$

After replacing $E, F, \beta, \gamma$ by suitable multiples, we obtain an equality

$$
[E]-[F]+\beta-\gamma \in \mu^{*} c_{1}(L) .
$$

We may further assume by subtracting that the divisors $E, F$ have no common components. The construction shows that $\beta^{2} \leqslant \operatorname{Vol}(X, L+t A)$ can be taken arbitrarily small (as well of course as $\gamma^{2}$ ), and the orthogonality estimate implies that we can assume $\beta \cdot E$ and $\gamma \cdot F$ to be arbitrarily small. Let us introduce metrics $h_{E}$ on $\mathscr{O}(E)$ and $h_{F}$ on $\mathscr{O}(F)$ as in Lemma 5.5, and consider the forms

$$
\begin{aligned}
u_{\varepsilon}= & +\frac{i}{2 \pi} \frac{\varepsilon^{2} D_{h_{E}}^{1,0} \sigma_{E} \wedge \overline{D_{h_{E}}^{1,0} \sigma_{E}}}{\left(\varepsilon^{2}+\left|\sigma_{E}\right|^{2}\right)^{2}}+\frac{\varepsilon^{2}}{\varepsilon^{2}+\left|\sigma_{E}\right|^{2}} \Theta_{E, h_{E}}+\beta \\
& -\frac{i}{2 \pi} \frac{\varepsilon^{2} D_{h_{F}}^{1,0} \sigma_{F} \wedge \overline{D_{h_{F}}^{1,0} \sigma_{F}}}{\left(\varepsilon^{2}+\left|\sigma_{F}\right|^{2}\right)^{2}}-\frac{\varepsilon^{2}}{\varepsilon^{2}+\left|\sigma_{F}\right|^{2}} \Theta_{F, h_{F}}-\gamma \in \mu^{*} c_{1}(L) .
\end{aligned}
$$


Observe that $u_{\varepsilon}$ converges uniformly to $\beta-\gamma$ outside of every neighborhood of $|E| \cup|F|$. Assume that $\Theta_{E, h_{E}}<0$ on $V_{E}=\left\{\left|\sigma_{E}\right|<\varepsilon_{0}\right\}$ and $\Theta_{F, h_{F}}<0$ on $V_{F}=\left\{\left|\sigma_{F}\right|<\varepsilon_{0}\right\}$. On $V_{E} \cup V_{F}$ we have

$$
u_{\varepsilon} \leqslant \frac{i}{2 \pi} \frac{\varepsilon^{2} D_{h_{E}}^{1,0} \sigma_{E} \wedge \overline{D_{h_{E}}^{1,0} \sigma_{E}}}{\left(\varepsilon^{2}+\left|\sigma_{E}\right|^{2}\right)^{2}}-\frac{\varepsilon^{2}}{\varepsilon^{2}+\left|\sigma_{F}\right|^{2}} \Theta_{F, h_{F}}+\beta+\frac{\varepsilon^{2}}{\varepsilon_{0}^{2}} \Theta_{E, h_{E}}^{+}
$$

where $\Theta_{E, h_{E}}^{+}$is the positive part of $\Theta_{E, h_{E}}$ with respect to $\beta$. One sees immediately that this term is negligible. The first term is the only one which is not uniformly bounded, and actually it converges weakly to the current $[E]$. By squaring, we find

$$
\limsup _{\varepsilon \rightarrow 0} \int_{X\left(u_{\varepsilon}, 0\right)} u_{\varepsilon}^{2} \leqslant \int_{X(\beta-\gamma, 0)}(\beta-\gamma)^{2}+2 \beta \cdot E .
$$

Notice that the term $-\frac{\varepsilon^{2}}{\varepsilon^{2}+\left|\sigma_{F}\right|^{2}} \Theta_{F, h_{F}}$ does not contribute to the limit as it converges boundedly almost everywhere to 0 , the exceptions being points of $|F|$, but this set is of measure zero with respect to the current $[E]$. Clearly we have $\int_{X(\beta-\gamma, 0)}(\beta-\gamma)^{2} \leqslant \beta^{2}$ and therefore

$$
\limsup _{\varepsilon \rightarrow 0} \int_{X\left(u_{\varepsilon}, 0\right)} u_{\varepsilon}^{2} \leqslant \beta^{2}+2 \beta \cdot E \text {. }
$$

Similarly, by looking at $-u_{\varepsilon}$, we find

$$
\limsup _{\varepsilon \rightarrow 0} \int_{X\left(u_{\varepsilon}, 2\right)} u_{\varepsilon}^{2} \leqslant \gamma^{2}+2 \gamma \cdot F .
$$

These limsup's are small and we conclude that the essential part of the mass is concentrated on the 1-index set, as desired.

(5.6) Remark. It is interesting to put these results in perspective with the algebraic version (I 2.14) of holomorphic Morse inequalities. When $X$ is projective, the algebraic Morse inequalities used in combination with the birational invariance of the Morse integrals imply the inequalities

$$
\begin{aligned}
& \inf _{u \in c_{1}(L)} \int_{X(u, q)}(-1)^{q} u^{n} \leq \inf _{\mu^{*}(L) \simeq \mathscr{O}(F-G)}\left(\begin{array}{l}
n \\
q
\end{array}\right) F^{n-q} \cdot G^{q}, \\
& \inf _{u \in c_{1}(L)} \int_{X(u, \leqslant q)}(-1)^{q} u^{n} \leq \inf _{\mu^{*}(L) \simeq \mathscr{G}(F-G)} \sum_{0 \leqslant j \leqslant q}(-1)^{q-j}\left(\begin{array}{c}
n \\
j
\end{array}\right) F^{n-j} \cdot G^{j},
\end{aligned}
$$

where the infimums on the right hand side are taken over all modifications $\mu: \widetilde{X} \rightarrow X$ and all decompositions $\mu^{*} L=\mathscr{O}(F-G)$ of $\mu^{*} L$ as a difference of two nef $\mathbb{Q}$-divisors $F, G$ on $\widetilde{X}$. Again, a natural question is to know whether these infimums derived from algebraic intersection numbers are equal to the asymptotic cohomology functionals $\widehat{h}^{q}(X, L)$ and $\widehat{h} \leq q(X, L)$. A positive answer would of course automatically yield a positive answer to the equality cases in 2.9 (a) and (b). However, the Zariski decompositions involved in our proofs of equality for $q=0$ or $n \leqslant 2$ produce certain effective exceptional divisors which are not nef. It is unclear how to write those effective divisors as a difference of nef divisors. This fact raises a lot of doubts upon the sufficiency of taking merely differences of nef divisors in the infimums 5.6 (a) and 5.6 (b), and it is likely that one needs a more subtle formula. 


\section{Singular holomorphic Morse inequalities}

The goal of this short section is to extend holomorphic Morse inequalities to the case of singular Hermitian metrics, following Bonavero's PhD thesis [Bon93] (cf. also [Bon98]).We always assume that our Hermitian metrics $h$ are given by quasi-psh weights $\varphi$. By Theorem (II 5.7), one can always approximate the weight by an arbitrary close quasi-psh weight $\varphi$ with analytic singularities, modulo smooth functions.

(6.1) Theorem. Let $(L, h)$ be a holomorphic line bundle on a compact complex $n$ fold $X$, and let $E$ be an arbitrary holomorphic vector bundle of rank $r$. Assume that locally $h=e^{-\varphi}$ has analytic singularities, and that $\varphi$ is quasi-psh of the form

$$
h=c \log \sum\left|g_{j}\right|^{2} \bmod C^{\infty}, \quad c>0
$$

in such a way that for a suitable modification $\mu: \widetilde{X} \rightarrow X$ one has $\mu^{*} \Theta_{L, h}=[D]+\beta$ where $D$ is an effective divisor and $\beta$ a smooth form on $\widetilde{X}$. Let $S=\mu(\operatorname{Supp} D)$ be the singular set of $h$. Then we have the following asymptotic estimates for the cohomology twisted by the appropriate multiplier ideal sheaves :

$$
\begin{aligned}
h^{q}\left(X, E \otimes L^{k} \otimes \mathscr{I}\left(h^{k}\right)\right) & \leqslant r \frac{k^{n}}{n !} \int_{X(L, h, q) \backslash S}(-1)^{q} \Theta_{L, h}^{n}+o\left(k^{n}\right) . \\
\sum_{0 \leqslant j \leqslant q}(-1)^{q-j} h^{j}\left(X, E \otimes L^{k} \otimes \mathscr{I}\left(h^{k}\right)\right) & \leqslant r \frac{k^{n}}{n !} \int_{X(L, h, \leqslant q) \backslash S}(-1)^{q} \Theta_{L, h}^{n}+o\left(k^{n}\right) .
\end{aligned}
$$

Proof. For this, we observe that the Morse integrals are given by

$$
\int_{\widetilde{X}(\beta, q)}(-1)^{q} \beta^{n},
$$

thanks to a change of variable $z=\mu(x)$. In fact, by our assumption $\Theta_{L, h}$ is smooth on $X \backslash S$, and its pull-back $\mu^{*} \Theta_{L, h}$ coincides with the smooth form $\beta$ on the complement $\widetilde{X} \backslash \operatorname{Supp} D$ (and Supp $D$ is a negligible set with respect to the integration of the smooth $(n, n)$ form $\beta^{n}$ on $\widetilde{X}$.) Now, a straightforward $L^{2}$ argument in the change of variable (cf. [Dem01]) yields the direct image formula

$$
K_{X} \otimes \mathscr{F}\left(h^{k}\right)=\mu_{*}\left(K_{\widetilde{X}} \otimes \mathscr{F}\left(\mu^{*} h^{k}\right)\right) .
$$

Let us introduce the relative canonical sheaf $K_{\widetilde{X} / X}=K_{\widetilde{X}} \otimes \mu^{*} K_{X}^{-1}=\mathscr{O}(\operatorname{div}(\operatorname{Jac} \mu))$ and let us put

$$
\widetilde{L}=\mu^{*} L, \quad \widetilde{h}=\mu^{*} h, \quad \widetilde{E}=\mu^{*} E \otimes K_{\widetilde{X} / X} .
$$

Then $\widetilde{h}$ has divisorial singularities and therefore $\mathscr{F}\left(\widetilde{h}^{k}\right)=\mathscr{G}(-\lfloor k D\rfloor)$ where $\lfloor\ldots\rfloor$ means the integral part of a divisor. The projection formula for direct images yields

$$
\begin{aligned}
\mu_{*}\left(\widetilde{E} \otimes \widetilde{L}^{k} \otimes \mathscr{F}\left(\widetilde{h}^{k}\right)\right) & =E \otimes L^{k} \otimes \mathscr{F}\left(h^{k}\right), \\
R^{q} \mu_{*}\left(\widetilde{E} \otimes \widetilde{L}^{k} \otimes \mathscr{F}\left(\widetilde{h}^{k}\right)\right) & =E \otimes L^{k} \otimes K_{X}^{-1} \otimes R^{q} \mu_{*}\left(K_{\widetilde{X}} \otimes \mathscr{F}\left(\widetilde{h}^{k}\right)\right) .
\end{aligned}
$$

However, for $k \geqslant k_{0}$ large enough, the multiplicities of $\lfloor k D\rfloor$ are all $>0$ for each of the components of $D$, hence $\mathscr{J}\left(h^{k}\right)=\mathscr{O}(-\lfloor k D\rfloor)$ is relatively ample with respect to the 
morphism $\mu: \widetilde{X} \rightarrow X$. From this, e.g. by an application of Hörmander's $L^{2}$ estimates (see [Bon93] for more details), we conclude that $R^{q} \mu_{*}\left(K_{\widetilde{X}} \otimes \mathscr{F}\left(\widetilde{h}^{k}\right)\right)=0$ for $k \geqslant k_{0}$. The Leray spectral sequence then implies

$$
H^{q}\left(X, E \otimes L^{k} \otimes \mathscr{I}\left(h^{k}\right)\right) \simeq H^{q}\left(\widetilde{X}, \widetilde{E} \otimes \widetilde{L}^{k} \otimes \mathscr{I}\left(\widetilde{h}^{k}\right)\right) .
$$

This reduces the proof to the case of divisorial singularities. Let us next assume that $D$ is a $\mathbb{Q}$-divisor. Let $a$ be a denominator for $D$, and put $k=a \ell+b, 0 \leqslant b \leqslant a-1$. Then

$$
\widetilde{E} \otimes \widetilde{L}^{k} \otimes \mathscr{F}\left(\widetilde{h}^{k}\right)=\widetilde{E} \otimes \widetilde{L}^{a \ell+b} \otimes \mathscr{G}(-a \ell D-\lfloor b D\rfloor)=F_{b} \otimes G^{\ell}
$$

where

$$
F_{b}=\widetilde{E} \otimes \widetilde{L}^{b} \otimes \mathscr{O}(-\lfloor b D\rfloor), \quad G=\widetilde{L}^{a} \otimes \mathscr{O}(-a D) .
$$

By construction, we get a smooth Hermitian metric $h_{G}$ on $G$ such that $\Theta_{G, h_{G}}=a \beta$. In this case, the proof is reduced to the standard case of holomorphic Morse inequalities, applied to the smooth Hermitian line bundle $\left(G, h_{G}\right)$ on $\widetilde{X}$ and the finite family of rank $r$ vector bundles $F_{b}, 0 \leqslant b \leqslant a-1$. The result is true even when $D$ is a real divisor. In fact, we can then perturb the coefficients of $D$ by small $\varepsilon$ 's to get a rational divisor $D_{\varepsilon}$, and we then have to change the smooth part of $\Theta_{\widetilde{L}, \widetilde{h}}$ to $\beta_{\varepsilon}=\beta+O(\varepsilon)$ (again smooth); actually $\beta_{\varepsilon}-\beta$ can be taken to be a linear combination by coefficients $O(\varepsilon)$ of given smooth forms representing the Chern classes $c_{1}\left(\mathscr{O}\left(D_{j}\right)\right)$ of the components of $D$. The Morse integrals are then perturbed by $O(\varepsilon)$. On the other hand, Theorem 1.9 shows that the cohomology groups in the right hand side of (6.3) are perturbed by $\varepsilon k^{n}$. The result follows as $\varepsilon \rightarrow 0$, thanks to the already settled rational case. 


\section{Part IV}

\section{Morse inequalities and the Green-Griffiths-Lang conjecture}

The goal of this section is to study the existence and properties of entire curves $f: \mathbb{C} \rightarrow X$ drawn in a complex irreducible $n$-dimensional variety $X$, and more specifically to show that they must satisfy certain global algebraic or differential equations as soon as $X$ is projective of general type. By means of holomorphic Morse inequalities and a probabilistic analysis of the cohomology of jet spaces, we are able to prove a significant step of a generalized version of the Green-Griffiths-Lang conjecture on the algebraic degeneracy of entire curves.

\section{Introduction}

Let $X$ be a complex $n$-dimensional manifold; most of the time we will assume that $X$ is compact and even projective algebraic. By an "entire curve" we always mean a non constant holomorphic map defined on the whole complex line $\mathbb{C}$, and we say that it is algebraically degenerate if its image is contained in a proper algebraic subvariety of the ambient variety. If $\mu: \widetilde{X} \rightarrow X$ is a modification and $f: \mathbb{C} \rightarrow X$ is an entire curve whose image $f(\mathbb{C})$ is not contained in the image $\mu(E)$ of the exceptional locus, then $f$ admits a unique lifting $\widetilde{f}: \mathbb{C} \rightarrow \widetilde{X}$. For this reason, the study of the algebraic degeneration of $f$ is a birationally invariant problem, and singularities do not play an essential role at this stage. We will therefore assume that $X$ is non singular, possibly after performing a suitable composition of blow-ups. We are interested more generally in the situation where the tangent bundle $T_{X}$ is equipped with a linear subspace $V \subset T_{X}$, that is, an irreducible complex analytic subset of the total space of $T_{X}$ such that

(0.1) all fibers $V_{x}:=V \cap T_{X, x}$ are vector subspaces of $T_{X, x}$.

Then the problem is to study entire curves $f: \mathbb{C} \rightarrow X$ which are tangent to $V$, i.e. such that $f_{*} T_{\mathbb{C}} \subset V$. We will refer to a pair $(X, V)$ as being a directed variety (or directed manifold $)$. A morphism of directed varieties $\Phi:(X, V) \rightarrow(Y, W)$ is a holomorphic map $\Phi: X \rightarrow Y$ such that $\Phi_{*} V \subset W$; by the irreducibility, it is enough to check this condition over the dense open subset $X \backslash \operatorname{Sing}(V)$ where $V$ is actually a subbundle. Here $\operatorname{Sing}(V)$ denotes the indeterminacy set of the associated meromorphic map $\alpha: X \rightarrow G_{r}\left(T_{X}\right)$ to the Grassmannian bbundle of $r$-planes in $T_{X}, r=\operatorname{rank} V$; we thus have $V_{\mid X \backslash \operatorname{Sing}(V)}=$ $\alpha^{*} S$ where $S \rightarrow G_{r}\left(T_{X}\right)$ is the tautological subbundle of $G_{r}\left(T_{X}\right)$. In that way, we get a category, and we will be mostly interested in the subcategory whose objects $(X, V)$ are projective algebraic manifolds equipped with algebraic linear subspaces. Notice that an entire curve $f: \mathbb{C} \rightarrow X$ tangent to $V$ is just a morphism $f:\left(\mathbb{C}, T_{\mathbb{C}}\right) \rightarrow(X, V)$. 
The case where $V=T_{X / S}$ is the relative tangent space of some fibration $X \rightarrow S$ is of special interest, and so is the case of a foliated variety (this is the situation where the sheaf of sections $\mathscr{O}(V)$ satisfies the Frobenius integrability condition $[\mathscr{O}(V), \mathscr{O}(V)] \subset \mathscr{O}(V))$; however, it is very useful to allow as well non integrable linear subspaces $V$. We refer to $V=T_{X}$ as being the absolute case. Our main target is the following deep conjecture concerning the algebraic degeneracy of entire curves, which generalizes similar statements made in [GG79] (see also [Lang86, Lang87]).

(0.2) Generalized Green-Griffiths-Lang conjecture. Let $(X, V)$ be a projective directed manifold such that the canonical sheaf $K_{V}$ is big (in the absolute case $V=T_{X}$, this means that $X$ is a variety of general type, and in the relative case we will say that $(X, V)$ is of general type). Then there should exist an algebraic subvariety $Y \subsetneq X$ such that every non constant entire curve $f: \mathbb{C} \rightarrow X$ tangent to $V$ is contained in $Y$.

The precise meaning of $K_{V}$ and of its bigness will be explained below - our definition does not coincide with other frequently used definitions and is in our view better suited to the study of entire curves of $(X, V)$. One says that $(X, V)$ is Brody-hyperbolic when there are no entire curves tangent to $V$. According to (generalized versions of) conjectures of Kobayashi [Kob70, Kob76] the hyperbolicity of $(X, V)$ should imply that $K_{V}$ is big, and even possibly ample, in a suitable sense. It would then follow from conjecture $(0.2)$ that $(X, V)$ is hyperbolic if and only if for every irreducible variety $Y \subset X$, the linear subspace $V_{\widetilde{Y}}=\overline{T_{\widetilde{Y} \backslash E} \cap \mu_{*}^{-1} V} \subset T_{\widetilde{Y}}$ has a big canonical sheaf whenever $\mu: \widetilde{Y} \rightarrow Y$ is a desingularization and $E$ is the exceptional locus.

The most striking fact known at this date on the Green-Griffiths-Lang conjecture is a recent result of Diverio, Merker and Rousseau [DMR10] in the absolute case, confirming the statement when $X \subset \mathbb{P}_{\mathbb{C}}^{n+1}$ is a generic non singular hypersurface of large degree $d$, with a (non optimal) sufficient lower bound $d \geqslant 2^{n^{5}}$. Their proof is based in an essential way on a strategy developed by Siu [Siu02, Siu04], combined with techniques of [Dem95]. Notice that if the Green-Griffiths-Lang conjecture holds true, a much stronger and probably optimal result would be true, namely all smooth hypersurfaces of degree $d \geqslant n+3$ would satisfy the expected algebraic degeneracy statement. Moreover, by results of Clemens [Cle86] and Voisin [Voi96], a (very) generic hypersurface of degree $d \geqslant 2 n+1$ would in fact be hyperbolic for every $n \geqslant 2$. Such a generic hyperbolicity statement has been obtained unconditionally by McQuillan [McQ98, McQ99] when $n=2$ and $d \geqslant 35$, and by Demailly-El Goul [DEG00] when $n=2$ and $d \geqslant 21$. Recently Diverio-Trapani [DT10] proved the same result when $n=3$ and $d \geqslant 593$. By definition, proving the algebraic degeneracy means finding a non zero polynomial $P$ on $X$ such that all entire curves $f: \mathbb{C} \rightarrow X$ satisfy $P(f)=0$. All known methods of proof are based on establishing first the existence of certain algebraic differential equations $P\left(f ; f^{\prime}, f^{\prime \prime}, \ldots, f^{(k)}\right)=0$ of some order $k$, and then trying to find enough such equations so that they cut out a proper algebraic locus $Y \subsetneq X$.

Let $J_{k} V$ be the space of $k$-jets of curves $f:(\mathbb{C}, 0) \rightarrow X$ tangent to $V$. One defines the sheaf $\mathscr{G}\left(E_{k, m}^{\mathrm{GG}} V^{*}\right)$ of jet differentials of order $k$ and degree $m$ to be the sheaf of holomorphic functions $P\left(z ; \xi_{1}, \ldots \xi_{k}\right)$ on $J_{k} V$ which are homogeneous polynomials of degree $m$ on the fibers of $J^{k} V \rightarrow X$ with respect to local coordinate derivatives $\xi_{j}=f^{(j)}(0)$ (see below in case $V$ has singularities). The degree $m$ considered here is the weighted degree with respect to the natural $\mathbb{C}^{*}$ action on $J^{k} V$ defined by $\lambda \cdot f(t):=f(\lambda t)$, i.e. by 
reparametrizing the curve with a homothetic change of variable. Since $(\lambda \cdot f)^{(j)}(t)=$ $\lambda^{j} f^{(j)}(\lambda t)$, the weighted action is given in coordinates by

$$
\lambda \cdot\left(\xi_{1}, \xi_{2}, \ldots, \xi_{k}\right)=\left(\lambda \xi_{1}, \lambda^{2} \xi_{2}, \ldots, \lambda^{k} \xi_{k}\right) .
$$

One of the major tool of the theory is the following result due to Green-Griffiths [GG79] (see also [Blo26], [Dem95, Dem97], [SY96a, SY96b], [Siu97]).

(0.4) Fundamental vanishing theorem. Let $(X, V)$ be a directed projective variety and $f:\left(\mathbb{C}, T_{\mathbb{C}}\right) \rightarrow(X, V)$ an entire curve tangent to $V$. Then for every global section $P \in H^{0}\left(X, E_{k, m}^{\mathrm{GG}} V^{*} \otimes \mathscr{O}(-A)\right)$ where $A$ is an ample divisor of $X$, one has $P\left(f ; f^{\prime}, f^{\prime \prime}, \ldots, f^{(k)}\right)=0$.

Let us give the proof of (0.4) in a special case. We interpret here $E_{k, m}^{\mathrm{GG}} V^{*} \otimes \mathscr{O}(-A)$ as the bundle of differential operators whose coefficients vanish along $A$. By a wellknown theorem of Brody [Bro78], for every entire curve $f:\left(\mathbb{C}, T_{\mathbb{C}}\right) \rightarrow(X, V)$, one can extract a convergent "renormalized" sequence $g=\lim f \circ h_{\nu}$ where $h_{\nu}$ are suitable homographic functions, in such a way that $g$ is an entire curve with bounded derivative $\sup _{t \in \mathbb{C}}\left\|g^{\prime}(t)\right\|_{\omega}<+\infty$ (with respect to any given Hermitian metric $\omega$ on $X$ ); the image $g(\mathbb{C})$ is then contained in the cluster set $\overline{f(\mathbb{C})}$, but it is possible that $\overline{g(\mathbb{C})} \subsetneq \overline{f(\mathbb{C})}$. Then Cauchy inequalities imply that all derivatives $g^{(j)}$ are bounded, and therefore, by compactness of $X, u=P\left(g ; g^{\prime}, g^{\prime \prime}, \ldots, g^{(k)}\right)$ is a bounded holomorphic function on $\mathbb{C}$. However, after raising $P$ to a power, we may assume that $A$ is very ample, and after moving $A \in|A|$, that $\operatorname{Supp} A$ intersects $g(\mathbb{C})$. Then $u$ vanishes somewhere, hence $u \equiv 0$ by Liouville's theorem. The proof for the general case is more subtle and makes use of Nevanlinna's second main theorem (see the above references).

It is expected that the global sections of $H^{0}\left(X, E_{k, m}^{\mathrm{GG}} V^{*} \otimes \sigma(-A)\right)$ are precisely those which ultimately define the algebraic locus $Y \subsetneq X$ where the curve $f$ should lie. The problem is then reduced to the question of showing that there are many non zero sections of $H^{0}\left(X, E_{k, m}^{\mathrm{GG}} V^{*} \otimes \mathcal{O}(-A)\right)$, and further, understanding what is their joint base locus. The first part of this program is the main result of this chapter.

(0.5) Theorem. Let $(X, V)$ be a directed projective variety such that $K_{V}$ is big and let $A$ be an ample divisor. Then for $k \gg 1$ and $\delta \in \mathbb{Q}_{+}$small enough, $\delta \leqslant c(\log k) / k$, the number of sections $h^{0}\left(X, E_{k, m}^{\mathrm{GG}} V^{*} \otimes \mathscr{O}(-m \delta A)\right)$ has maximal growth, i.e. is larger that $c_{k} m^{n+k r-1}$ for some $m \geqslant m_{k}$, where $c, c_{k}>0, n=\operatorname{dim} X$ and $r=\operatorname{rank} V$. In particular, entire curves $f:\left(\mathbb{C}, T_{\mathbb{C}}\right) \rightarrow(X, V)$ satisfy (many) algebraic differential equations.

The statement is very elementary to check when $r=\operatorname{rank} V=1$, and therefore when $n=\operatorname{dim} X=1$. In higher dimensions $n \geqslant 2$, only very partial results were known at this point, concerning merely the absolute case $V=T_{X}$. In dimension 2 , Theorem 0.5 is a consequence of the Riemann-Roch calculation of Green-Griffiths [GG79], combined with a vanishing theorem due to Bogomolov [Bog79] - the latter actually only applies to the top cohomology group $H^{n}$, and things become much more delicate when extimates of intermediate cohomology groups are needed. In higher dimensions, Diverio [Div08, Div09] proved the existence of sections of $H^{0}\left(X, E_{k, m}^{\mathrm{GG}} V^{*} \otimes \mathscr{G}(-1)\right)$ whenever $X$ is a hypersurface of $\mathbb{P}_{\mathbb{C}}^{n+1}$ of high degree $d \geqslant d_{n}$, assuming $k \geqslant n$ and $m \geqslant m_{n}$. More recently, Merker [Mer10] was able to treat the case of arbitrary hypersurfaces of general type, i.e. 
$d \geqslant n+3$, assuming this time $k$ to be very large. The latter result is obtained through explicit algebraic calculations of the spaces of sections, and the proof is computationally very intensive. Bérczi [Ber10] also obtained related results with a different approach based on residue formulas, assuming $d \geqslant 2^{7 n \log n}$.

All these approaches are algebraic in nature, and use only the algebraic version of holomorphic Morse inequalities (section I 2.D). Here, however, our techniques are based on more elaborate curvature estimates in the spirit of Cowen-Griffiths [CG76]. They require the stronger analytic form of holomorphic Morse inequalities (see Chapter I and Section III 6) - and we do not know how to translate our method in an algebraic setting. Notice that holomorphic Morse inequalities are essentially insensitive to singularities, as we can pass to non singular models and blow-up $X$ as much as we want: if $\mu: \widetilde{X} \rightarrow X$ is a modification then $\mu_{*} \mathscr{\sigma}_{\widetilde{X}}=\mathscr{\sigma}_{X}$ and $R^{q} \mu_{*} \mathscr{\sigma}_{\widetilde{X}}$ is supported on a codimension 1 analytic subset (even codimension 2 if $X$ is smooth). As already observed in Section III 3, it follows from the Leray spectral sequence that the cohomology estimates for $L$ on $X$ or for $\widetilde{L}=\mu^{*} L$ on $\widetilde{X}$ differ by negligible terms, i.e.

$$
h^{q}\left(\widetilde{X}, \widetilde{L}^{\otimes m}\right)-h^{q}\left(X, L^{\otimes m}\right)=O\left(m^{n-1}\right) .
$$

Finally, singular holomorphic Morse inequalities (see Setion III 6) allow us to work with singular Hermitian metrics $h$; this is the reason why we will only require to have big line bundles rather than ample line bundles. In the case of linear subspaces $V \subset T_{X}$, we introduce singular Hermitian metrics as follows.

(0.6) Definition. A singular Hermitian metric on a linear subspace $V \subset T_{X}$ is a metric $h$ on the fibers of $V$ such that the function $\log h: \xi \mapsto \log |\xi|_{h}^{2}$ is locally integrable on the total space of $V$.

Such a metric can also be viewed as a singular Hermitian metric on the tautological line bundle $\mathscr{G}_{P(V)}(-1)$ on the projectivized bundle $P(V)=V \backslash\{0\} / \mathbb{C}^{*}$, and therefore its dual metric $h^{*}$ defines a curvature current $\Theta_{\Theta_{P(V)}(1), h^{*}}$ of type $(1,1)$ on $P(V) \subset P\left(T_{X}\right)$, such that

$$
p^{*} \Theta_{\Theta_{P(V)}(1), h^{*}}=\frac{i}{2 \pi} \partial \bar{\partial} \log h, \quad \text { where } p: V \backslash\{0\} \rightarrow P(V) .
$$

If $\log h$ is quasi-plurisubharmonic (or quasi-psh, which means psh modulo addition of a smooth function) on $V$, then $\log h$ is indeed locally integrable, and we have moreover

$$
\Theta_{\Theta_{P(V)}}(1), h^{*} \geqslant-C \omega
$$

for some smooth positive $(1,1)$-form on $P(V)$ and some constant $C>0$; conversely, if (0.7) holds, then $\log h$ is quasi-psh.

(0.8) Definition. We will say that a singular Hermitian metric $h$ on $V$ is admissible if $h$ can be written as $h=e^{\varphi} h_{0 \mid V}$ where $h_{0}$ is a smooth positive definite Hermitian on $T_{X}$ and $\varphi$ is a quasi-psh weight with analytic singularities on $X$, as in (0.6). Then $h$ can be seen as a singular Hermitian metric on $\mathscr{O}_{P(V)}(1)$, with the property that it induces a smooth positive definite metric on a Zariski open set $X^{\prime} \subset X \backslash \operatorname{Sing}(V)$; we will denote by $\operatorname{Sing}(h) \supset \operatorname{Sing}(V)$ the complement of the largest such Zariski open set $X^{\prime}$. 
If $h$ is an admissible metric, we define $\sigma_{h}\left(V^{*}\right)$ to be the sheaf of germs of holomorphic sections sections of $V_{\mid X \backslash \operatorname{Sing}(h)}^{*}$ which are $h^{*}$-bounded near $\operatorname{Sing}(h)$; by the assumption on the analytic singularities, this is a coherent sheaf (as the direct image of some coherent sheaf on $P(V)$ ), and actually, since $h^{*}=e^{-\varphi} h_{0}^{*}$, it is a subsheaf of the sheaf $\mathscr{G}\left(V^{*}\right):=\mathscr{O}_{h_{0}}\left(V^{*}\right)$ associated with a smooth positive definite metric $h_{0}$ on $T_{X}$. If $r$ is the generic rank of $V$ and $m$ a positive integer, we define similarly $K_{V, h}^{m}$ to be sheaf of germs of holomorphic sections of $\left(\operatorname{det} V_{\mid X^{\prime}}^{*}\right)^{\otimes m}=\left(\Lambda^{r} V_{\mid X^{\prime}}^{*}\right)^{\otimes m}$ which are $\operatorname{det} h^{*}$-bounded, and $K_{V}^{m}:=K_{V, h_{0}}^{m}$.

If $V$ is defined by $\alpha: X \rightarrow G_{r}\left(T_{X}\right)$, there always exists a modification $\mu: \widetilde{X} \rightarrow X$ such that the composition $\alpha \circ \mu: \widetilde{X} \rightarrow G_{r}\left(\mu^{*} T_{X}\right)$ becomes holomorphic, and then $\mu^{*} V_{\mid \mu^{-1}(X \backslash \operatorname{Sing}(V))}$ extends as a locally trivial subbundle of $\mu^{*} T_{X}$ which we will simply denote by $\mu^{*} V$. If $h$ is an admissible metric on $V$, then $\mu^{*} V$ can be equipped with the metric $\mu^{*} h=e^{\varphi \circ \mu} \mu^{*} h_{0}$ where $\mu^{*} h_{0}$ is smooth and positive definite. We may assume that $\varphi \circ \mu$ has divisorial singularities (otherwise just perform further blow-ups of $\widetilde{X}$ to achieve this). We then see that there is an integer $m_{0}$ such that for all multiples $m=p m_{0}$ the pull-back $\mu^{*} K_{V, h}^{m}$ is an invertible sheaf on $\widetilde{X}$, and $\operatorname{det} h^{*}$ induces a smooth non singular metric on it (when $h=h_{0}$, we can even take $m_{0}=1$ ). By definition we always have $K_{V, h}^{m}=\mu_{*}\left(\mu^{*} K_{V, h}^{m}\right)$ for any $m \geqslant 0$. In the sequel, however, we think of $K_{V, h}$ not really as a coherent sheaf, but rather as the "virtual" $\mathbb{Q}$-line bundle $\mu_{*}\left(\mu^{*} K_{V, h}^{m_{0}}\right)^{1 / m_{0}}$, and we say that $K_{V, h}$ is big if $h^{0}\left(X, K_{V, h}^{m}\right) \geqslant c m^{n}$ for $m \geqslant m_{1}$, with $c>0$, i.e. if the invertible sheaf $\mu^{*} K_{V, h}^{m_{0}}$ is big in the usual sense.

At this point, it is important to observe that "our" canonical sheaf $K_{V}$ differs from the sheaf $\mathscr{K}_{V}:=i_{*} \mathscr{O}\left(K_{V}\right)$ associated with the injection $i: X \backslash \operatorname{Sing}(V) \hookrightarrow X$, which is usually referred to as being the "canonical sheaf", at least when $V$ is the space of tangents to a foliation. In fact, $\mathscr{K}_{V}$ is always an invertible sheaf and there is an obvious inclusion $K_{V} \subset \mathscr{K}_{V}$. More precisely, the image of $\mathscr{G}\left(\Lambda^{r} T_{X}^{*}\right) \rightarrow \mathscr{K}_{V}$ is equal to $\mathscr{K}_{V} \otimes_{\mathscr{O}_{X}} \mathcal{F}$ for a certain coherent ideal $\mathscr{J} \subset \mathscr{O}_{X}$, and the condition to have $h_{0}$-bounded sections on $X \backslash \operatorname{Sing}(V)$ precisely means that our sections are bounded by Const $\sum\left|g_{j}\right|$ in terms of the generators $\left(g_{j}\right)$ of $\mathscr{K}_{V} \otimes_{\Theta_{X}} \mathscr{J}$, i.e. $K_{V}=\mathscr{K}_{V} \otimes_{\Theta_{X}} \overline{\mathcal{J}}$ where $\overline{\mathcal{J}}$ is the integral closure of $\mathscr{f}$. More generally,

$$
K_{V, h}^{m}=\mathscr{K}_{V}^{m} \otimes_{\Theta_{X}} \overline{\mathscr{J}}_{h, m_{0}}^{m / m_{0}}
$$

where $\overline{\mathscr{F}}_{h, m_{0}}^{m / m_{0}} \subset \mathscr{O}_{X}$ is the $\left(m / m_{0}\right)$-integral closure of a certain ideal sheaf $\mathscr{F}_{h, m_{0}} \subset \mathscr{O}_{X}$, which can itself be assumed to be integrally closed; in our previous discussion, $\mu$ is chosen so that $\mu^{*} \mathscr{J}_{h, m_{0}}$ is invertible on $\widetilde{X}$.

The discrepancy already occurs e.g. with the rank 1 linear space $V \subset T_{\mathbb{P}_{\mathbb{C}}^{n}}$ consisting at each point $z \neq 0$ of the tangent to the line $(0 z)$ (so that necessarily $V_{0}=\breve{T}_{\mathbb{P}_{\mathbb{C}}^{n}, 0}$ ). As a sheaf (and not as a linear space), $i_{*} \mathscr{O}(V)$ is the invertible sheaf generated by the vector field $\xi=\sum z_{j} \partial / \partial z_{j}$ on the affine open set $\mathbb{C}^{n} \subset \mathbb{P}_{\mathbb{C}}^{n}$, and therefore $\mathscr{K}_{V}:=i_{*} \mathscr{O}\left(V^{*}\right)$ is generated over $\mathbb{C}^{n}$ by the unique 1 -form $u$ such that $u(\xi)=1$. Since $\xi$ vanishes at 0 , the generator $u$ is unbounded with respect to a smooth metric $h_{0}$ on $T_{\mathbb{P}_{\mathbb{C}}^{n}}$, and it is easily seen that $K_{V}$ is the non invertible sheaf $K_{V}=\mathscr{K}_{V} \otimes \mathfrak{m}_{\mathbb{P}_{\mathbb{C}}^{n}, 0}$. We can make it invertible by considering the blow-up $\mu: \widetilde{X} \rightarrow X$ of $X=\mathbb{P}_{\mathbb{C}}^{n}$ at 0 , so that $\mu^{*} K_{V}$ is isomorphic to $\mu^{*} \mathscr{K}_{V} \otimes \mathscr{G}_{\widetilde{X}}(-E)$ where $E$ is the exceptional divisor. The integral curves $C$ of $V$ are of course lines through 0 , and when a standard parametrization is used, their derivatives do not vanish at 0 , while the sections of $i_{*} \mathscr{G}(V)$ do - another sign that $i_{*} \mathscr{G}(V)$ and $i_{*} \mathscr{G}\left(V^{*}\right)$ are the wrong objects to consider. Another standard example is obtained by taking a 
generic pencil of elliptic curves $\lambda P(z)+\mu Q(z)=0$ of degree 3 in $\mathbb{P}_{\mathbb{C}}^{2}$, and the linear space $V$ consisting of the tangents to the fibers of the rational map $\mathbb{P}_{\mathbb{C}}^{2} \rightarrow-\rightarrow \mathbb{P}_{\mathbb{C}}^{1}$ defined by $z \mapsto Q(z) / P(z)$. Then $V$ is given by

$$
0 \longrightarrow i_{*} \mathscr{G}(V) \longrightarrow \mathscr{O}\left(T_{\mathbb{P}_{\mathbb{C}}^{2}}\right) \stackrel{P d Q-Q d P}{\longrightarrow} \mathscr{O}_{\mathbb{P}_{\mathbb{C}}^{2}}(6) \otimes \mathscr{F}_{S} \longrightarrow 0
$$

where $S=\operatorname{Sing}(V)$ consists of the 9 points $\{P(z)=0\} \cap\{Q(z)=0\}$, and $\mathscr{F}_{S}$ is the corresponding ideal sheaf of $S$. Since $\operatorname{det} \mathscr{O}\left(T_{\mathbb{P}^{2}}\right)=\mathscr{O}(3)$, we see that $\mathscr{K}_{V}=\mathscr{O}(3)$ is ample, which seems to contradict (0.2) since all leaves are elliptic curves. There is however no such contradiction, because $K_{V}=\mathscr{K}_{V} \otimes \mathscr{F}_{S}$ is not big in our sense (it has degree 0 on all members of the elliptic pencil). A similar example is obtained with a generic pencil of conics, in which case $\mathscr{K}_{V}=\mathscr{O}(1)$ and card $S=4$.

For a given admissible Hermitian structure $(V, h)$, we define similarly the sheaf $E_{k, m}^{\mathrm{GG}} V_{h}^{*}$ to be the sheaf of polynomials defined over $X \backslash \operatorname{Sing}(h)$ which are " $h$-bounded". This means that when they are viewed as polynomials $P\left(z ; \xi_{1}, \ldots, \xi_{k}\right)$ in terms of $\xi_{j}=\left(\nabla_{h_{0}}^{1,0}\right)^{j} f(0)$ where $\nabla_{h_{0}}^{1,0}$ is the $(1,0)$-component of the induced Chern connection on $\left(V, h_{0}\right)$, there is a uniform bound

$$
\left|P\left(z ; \xi_{1}, \ldots, \xi_{k}\right)\right| \leqslant C\left(\sum\left\|\xi_{j}\right\|_{h}^{1 / j}\right)^{m}
$$

near points of $X \backslash X^{\prime}$ (see section 2 for more details on this). Again, by a direct image argument, one sees that $E_{k, m}^{\mathrm{GG}} V_{h}^{*}$ is always a coherent sheaf. The sheaf $E_{k, m}^{\mathrm{GG}} V^{*}$ is defined to be $E_{k, m}^{\mathrm{GG}} V_{h}^{*}$ when $h=h_{0}$ (it is actually independent of the choice of $h_{0}$, as follows from arguments similar to those given in section 2). Notice that this is exactly what is needed to extend the proof of the vanishing theorem 0.4 to the case of a singular linear space $V$; the value distribution theory argument can only work when the functions $P\left(f ; f^{\prime}, \ldots, f^{(k)}\right)(t)$ do not exhibit poles, and this is guaranteed here by the boundedness assumption.

Our strategy can be described as follows. We consider the Green-Griffiths bundle of $k$-jets $X_{k}^{\mathrm{GG}}=J^{k} V \backslash\{0\} / \mathbb{C}^{*}$, which by $(0.3)$ consists of a fibration in weighted projective spaces, and its associated tautological sheaf

$$
L=\mathscr{\sigma}_{X_{k}^{\mathrm{GG}}}(1)
$$

viewed rather as a virtual $\mathbb{Q}$-line bundle $\mathscr{C}_{X_{k}^{\mathrm{GG}}}\left(m_{0}\right)^{1 / m_{0}}$ with $m_{0}=\operatorname{lcm}(1,2, \ldots, k)$. Then, if $\pi_{k}: X_{k}^{\mathrm{GG}} \rightarrow X$ is the natural projection, we have

$$
E_{k, m}^{\mathrm{GG}}=\left(\pi_{k}\right)_{*} \mathscr{O}_{X_{k}^{\mathrm{GG}}}(m) \text { and } R^{q}\left(\pi_{k}\right)_{*} \mathscr{O}_{X_{k}^{\mathrm{GG}}}(m)=0 \text { for } q \geqslant 1 .
$$

Hence, by the Leray spectral sequence we get for every invertible sheaf $F$ on $X$ the isomorphism

$$
H^{q}\left(X, E_{k, m}^{\mathrm{GG}} V^{*} \otimes F\right) \simeq H^{q}\left(X_{k}^{\mathrm{GG}}, \mathscr{\sigma}_{X_{k}^{\mathrm{GG}}}(m) \otimes \pi_{k}^{*} F\right)
$$

The latter group can be evaluated thanks to holomorphic Morse inequalities. In fact we can associate with any admissible metric $h$ on $V$ a metric (or rather a natural family) of metrics on $L=\mathscr{G}_{X_{k}^{\mathrm{GG}}}(1)$. The space $X_{k}^{\mathrm{GG}}$ always possesses quotient singularities if 
$k \geqslant 2$ (and even some more if $V$ is singular), but we do not really care since Morse inequalities still work in this setting. As we will see, it is then possible to get nice asymptotic formulas as $k \rightarrow+\infty$. They appear to be of a probabilistic nature if we take the components of the $k$-jet (i.e. the successive derivatives $\xi_{j}=f^{(j)}(0), 1 \leqslant j \leqslant k$ ) as random variables. This probabilistic behaviour was somehow already visible in the Riemann-Roch calculation of [GG79]. In this way, assuming $K_{V}$ big, we produce a lot of sections $\sigma_{j}=H^{0}\left(X_{k}^{\mathrm{GG}}, \mathscr{O}_{X_{k}^{\mathrm{GG}}}(m) \otimes \pi_{k}^{*} F\right)$, corresponding to certain divisors $Z_{j} \subset X_{k}^{\mathrm{GG}}$. The hard problem which is left in order to complete a proof of the generalized GreenGriffiths-Lang conjecture is to compute the base locus $Z=\bigcap Z_{j}$ and to show that $Y=\pi_{k}(Z) \subset X$ must be a proper algebraic variety. Unfortunately we cannot address this problem at present.

\section{Hermitian geometry of weighted projective spaces}

The goal of this section is to introduce natural Kähler metrics on weighted projective spaces, and to evaluate the corresponding volume forms. Here we put $d^{c}=\frac{i}{4 \pi}(\bar{\partial}-\partial)$ so that $d d^{c}=\frac{i}{2 \pi} \partial \bar{\partial}$. The normalization of the $d^{c}$ operator is chosen such that we have precisely $\left(d d^{c} \log |z|^{2}\right)^{n}=\delta_{0}$ for the Monge-Ampère operator in $\mathbb{C}^{n}$; also, for every holomorphic or meromorphic section $\sigma$ of a Hermitian line bundle $(L, h)$ the LelongPoincaré can be formulated

$$
d d^{c} \log |\sigma|_{h}^{2}=\left[Z_{\sigma}\right]-\Theta_{L, h},
$$

where $\Theta_{L, h}=\frac{i}{2 \pi} D_{L, h}^{2}$ is the $(1,1)$-curvature form of $L$ and $Z_{\sigma}$ the zero divisor of $\sigma$. The closed $(1,1)$-form $\Theta_{L, h}$ is a representative of the first Chern class $c_{1}(L)$. Given a $k$-tuple of "weights" $a=\left(a_{1}, \ldots, a_{k}\right)$, i.e. of integers $a_{s}>0$ with $\operatorname{gcd}\left(a_{1}, \ldots, a_{k}\right)=1$, we introduce the weighted projective space $P\left(a_{1}, \ldots, a_{k}\right)$ to be the quotient of $\mathbb{C}^{k} \backslash\{0\}$ by the corresponding weighted $\mathbb{C}^{*}$ action:

$$
P\left(a_{1}, \ldots, a_{k}\right)=\mathbb{C}^{k} \backslash\{0\} / \mathbb{C}^{*}, \quad \lambda \cdot z=\left(\lambda^{a_{1}} z_{1}, \ldots, \lambda^{a_{k}} z_{k}\right) .
$$

As is well known, this defines a toric $(k-1)$-dimensional algebraic variety with quotient singularities. On this variety, we introduce the possibly singular (but almost everywhere smooth and non degenerate) Kähler form $\omega_{a, p}$ defined by

$$
\pi_{a}^{*} \omega_{a, p}=d d^{c} \varphi_{a, p}, \quad \varphi_{a, p}(z)=\frac{1}{p} \log \sum_{1 \leqslant s \leqslant k}\left|z_{s}\right|^{2 p / a_{s}},
$$

where $\pi_{a}: \mathbb{C}^{k} \backslash\{0\} \rightarrow P\left(a_{1}, \ldots, a_{k}\right)$ is the canonical projection and $p>0$ is a positive constant. It is clear that $\varphi_{p, a}$ is real analytic on $\mathbb{C}^{k} \backslash\{0\}$ if $p$ is an integer and a common multiple of all weights $a_{s}$. It is at least $C^{2}$ if $p$ is real and $p \geqslant \max \left(a_{s}\right)$, which will be more than sufficient for our purposes (but everything would still work for any $p>0$ ). The resulting metric is in any case smooth and positive definite outside of the coordinate hyperplanes $z_{s}=0$, and these hyperplanes will not matter here since they are of capacity zero with respect to all currents $\left(d d^{c} \varphi_{a, p}\right)^{\ell}$. In order to evaluate the volume $\int_{P\left(a_{1}, \ldots, a_{k}\right)} \omega_{a, p}^{k-1}$, one can observe that

$$
\int_{P\left(a_{1}, \ldots, a_{k}\right)} \omega_{a, p}^{k-1}=\int_{z \in \mathbb{C}^{k}, \varphi_{a, p}(z)=0} \pi_{a}^{*} \omega_{a, p}^{k-1} \wedge d^{c} \varphi_{a, p}
$$




$$
\begin{aligned}
& =\int_{z \in \mathbb{C}^{k}, \varphi_{a, p}(z)=0}\left(d d^{c} \varphi_{a, p}\right)^{k-1} \wedge d^{c} \varphi_{a, p} \\
& =\frac{1}{p^{k}} \int_{z \in \mathbb{C}^{k}, \varphi_{a, p}(z)<0}\left(d d^{c} e^{p \varphi_{a, p}}\right)^{k} .
\end{aligned}
$$

The first equality comes from the fact that $\left\{\varphi_{a, p}(z)=0\right\}$ is a circle bundle over $P\left(a_{1}, \ldots, a_{k}\right)$, together with the identities $\varphi_{a, p}(\lambda \cdot z)=\varphi_{a, p}(z)+\log |\lambda|^{2}$ and $\int_{|\lambda|=1} d^{c} \log |\lambda|^{2}=1$. The third equality can be seen by Stokes formula applied to the $(2 k-1)$-form

$$
\left(d d^{c} e^{p \varphi_{a, p}}\right)^{k-1} \wedge d^{c} e^{p \varphi_{a, p}}=e^{p \varphi_{a, p}}\left(d d^{c} \varphi_{a, p}\right)^{k-1} \wedge d^{c} \varphi_{a, p}
$$

on the pseudoconvex open set $\left\{z \in \mathbb{C}^{k} ; \varphi_{a, p}(z)<0\right\}$. Now, we find

$$
\begin{aligned}
\left(d d^{c} e^{p \varphi_{a, p}}\right)^{k}=\left(d d^{c} \sum_{1 \leqslant s \leqslant k}\left|z_{s}\right|^{2 p / a_{s}}\right)^{k} & =\prod_{1 \leqslant s \leqslant k}\left(\frac{p}{a_{s}}\left|z_{s}\right|^{\frac{p}{a_{s}}-1}\right)\left(d d^{c}|z|^{2}\right)^{k} \\
\int_{z \in \mathbb{C}^{k}, \varphi_{a, p}(z)<0}\left(d d^{c} e^{p \varphi_{a, p}}\right)^{k} & =\prod_{1 \leqslant s \leqslant k} \frac{p}{a_{s}}=\frac{p^{k}}{a_{1} \ldots a_{k}} .
\end{aligned}
$$

In fact, (1.5) and (1.6) are clear when $p=a_{1}=\ldots=a_{k}=1$ (this is just the standard calculation of the volume of the unit ball in $\left.\mathbb{C}^{k}\right)$; the general case follows by substituting formally $z_{s} \mapsto z_{s}^{p / a_{s}}$, and using rotational invariance together with the observation that the arguments of the complex numbers $z_{s}^{p / a_{s}}$ now run in the interval $\left[0,2 \pi p / a_{s}\right.$ [ instead of $[0,2 \pi[$ (say). As a consequence of (1.4) and (1.6), we obtain the well known value

$$
\int_{P\left(a_{1}, \ldots, a_{k}\right)} \omega_{a, p}^{k-1}=\frac{1}{a_{1} \ldots a_{k}}
$$

for the volume. Notice that this is independent of $p$ (as it is obvious by Stokes theorem, since the cohomology class of $\omega_{a, p}$ does not depend on $p$ ). When $p$ tends to $+\infty$, we have $\varphi_{a, p}(z) \mapsto \varphi_{a, \infty}(z)=\log \max _{1 \leqslant s \leqslant k}\left|z_{s}\right|^{2 / a_{s}}$ and the volume form $\omega_{a, p}^{k-1}$ converges to a rotationally invariant measure supported by the image of the polycircle $\prod\left\{\left|z_{s}\right|=1\right\}$ in $P\left(a_{1}, \ldots, a_{k}\right)$. This is so because not all $\left|z_{s}\right|^{2 / a_{s}}$ are equal outside of the image of the polycircle, thus $\varphi_{a, \infty}(z)$ locally depends only on $k-1$ complex variables, and so $\omega_{a, \infty}^{k-1}=0$ there by log homogeneity.

Our later calculations will require a slightly more general setting. Instead of looking at $\mathbb{C}^{k}$, we consider the weighted $\mathbb{C}^{*}$ action defined by

$$
\mathbb{C}^{|r|}=\mathbb{C}^{r_{1}} \times \ldots \times \mathbb{C}^{r_{k}}, \quad \lambda \cdot z=\left(\lambda^{a_{1}} z_{1}, \ldots, \lambda^{a_{k}} z_{k}\right) .
$$

Here $z_{s} \in \mathbb{C}^{r_{s}}$ for some $k$-tuple $r=\left(r_{1}, \ldots, r_{k}\right)$ and $|r|=r_{1}+\ldots+r_{k}$. This gives rise to a weighted projective space

$$
\begin{aligned}
& P\left(a_{1}^{\left[r_{1}\right]}, \ldots, a_{k}^{\left[r_{k}\right]}\right)=P\left(a_{1}, \ldots, a_{1}, \ldots, a_{k}, \ldots, a_{k}\right), \\
& \pi_{a, r}: \mathbb{C}^{r_{1}} \times \ldots \times \mathbb{C}^{r_{k}} \backslash\{0\} \longrightarrow P\left(a_{1}^{\left[r_{1}\right]}, \ldots, a_{k}^{\left[r_{k}\right]}\right)
\end{aligned}
$$


obtained by repeating $r_{s}$ times each weight $a_{s}$. On this space, we introduce the degenerate Kähler metric $\omega_{a, r, p}$ such that

$$
\pi_{a, r}^{*} \omega_{a, r, p}=d d^{c} \varphi_{a, r, p}, \quad \varphi_{a, r, p}(z)=\frac{1}{p} \log \sum_{1 \leqslant s \leqslant k}\left|z_{s}\right|^{2 p / a_{s}}
$$

where $\left|z_{s}\right|$ stands now for the standard Hermitian norm $\left(\sum_{1 \leqslant j \leqslant r_{s}}\left|z_{s, j}\right|^{2}\right)^{1 / 2}$ on $\mathbb{C}^{r_{s}}$. This metric is cohomologous to the corresponding "polydisc-like" metric $\omega_{a, p}$ already defined, and therefore Stokes theorem implies

$$
\int_{P\left(a_{1}^{\left[r_{1}\right]}, \ldots, a_{k}^{\left[r_{k}\right]}\right)} \omega_{a, r, p}^{|r|-1}=\frac{1}{a_{1}^{r_{1}} \ldots a_{k}^{r_{k}}} .
$$

Since $\left(d d^{c} \log \left|z_{s}\right|^{2}\right)^{r_{s}}=0$ on $\mathbb{C}^{r_{s}} \backslash\{0\}$ by homogeneity, we conclude as before that the weak $\operatorname{limit}_{\lim _{p \rightarrow+\infty}} \omega_{a, r, p}^{|r|-1}=\omega_{a, r, \infty}^{|r|-1}$ associated with

$$
\varphi_{a, r, \infty}(z)=\log \max _{1 \leqslant s \leqslant k}\left|z_{s}\right|^{2 / a_{s}}
$$

is a measure supported by the image of the product of unit spheres $\prod S^{2 r_{s}-1}$ in $P\left(a_{1}^{\left[r_{1}\right]}, \ldots, a_{k}^{\left[r_{k}\right]}\right)$, which is invariant under the action of $U\left(r_{1}\right) \times \ldots \times U\left(r_{k}\right)$ on $\mathbb{C}^{r_{1}} \times \ldots \times \mathbb{C}^{r_{k}}$, and thus coincides with the Hermitian area measure up to a constant determined by condition (1.11). In fact, outside of the product of spheres, $\varphi_{a, r, \infty}$ locally depends only on at most $k-1$ factors and thus, for dimension reasons, the top power $\left(d d^{c} \varphi_{a, r, \infty}\right)^{|r|-1}$ must be zero there. In the next section, the following change of variable formula will be needed. For simplicity of exposition we restrict ourselves to continuous functions, but a standard density argument would easily extend the formula to all functions that are Lebesgue integrable with respect to the volume form $\omega_{a, r, p}^{|r|-1}$.

(1.13) Proposition. Let $f(z)$ be a bounded function on $P\left(a_{1}^{\left[r_{1}\right]}, \ldots, a_{k}^{\left[r_{k}\right]}\right)$ which is continuous outside of the hyperplane sections $z_{s}=0$. We also view $f$ as a $\mathbb{C}^{*}$-invariant continuous function on $\prod\left(\mathbb{C}^{r_{s}} \backslash\{0\}\right)$. Then

$$
\begin{aligned}
& \int_{P\left(a_{1}^{\left[r_{1}\right]}, \ldots, a_{k}^{\left[r_{k}\right]}\right)} f(z) \omega_{a, r, p}^{|r|-1} \\
& =\frac{(|r|-1) !}{\prod_{s} a_{s}^{r_{s}}} \int_{(x, u) \in \Delta_{k-1} \times} \prod_{S^{2 r_{s}-1}} f\left(x_{1}^{a_{1} / 2 p} u_{1}, \ldots, x_{k}^{a_{k} / 2 p} u_{k}\right) \prod_{1 \leqslant s \leqslant k} \frac{x_{s}^{r_{s}-1}}{\left(r_{s}-1\right) !} d x d \mu(u)
\end{aligned}
$$

where $\Delta_{k-1}$ is the $(k-1)$-simplex $\left\{x_{s} \geqslant 0, \sum x_{s}=1\right\}, d x=d x_{1} \wedge \ldots \wedge d x_{k-1}$ its standard measure, and where $d \mu(u)=d \mu_{1}\left(u_{1}\right) \ldots d \mu_{k}\left(u_{k}\right)$ is the rotation invariant probability measure on the product $\prod_{s} S^{2 r_{s}-1}$ of unit spheres in $\mathbb{C}^{r_{1}} \times \ldots \times \mathbb{C}^{r_{k}}$. As a consequence

$$
\lim _{p \rightarrow+\infty} \int_{P\left(a_{1}^{\left[r_{1}\right]}, \ldots, a_{k}^{\left[r_{k}\right]}\right)} f(z) \omega_{a, r, p}^{|r|-1}=\frac{1}{\prod_{s} a_{s}^{r_{s}}} \int_{\prod_{S^{2 r_{s}-1}}} f(u) d \mu(u) .
$$

Proof. The area formula of the disc $\int_{|\lambda|<1} d d^{c}|\lambda|^{2}=1$ and a consideration of the unit disc bundle over $P\left(a_{1}^{\left[r_{1}\right]}, \ldots, a_{k}^{\left[r_{k}\right]}\right)$ imply that

$$
I_{p}:=\int_{P\left(a_{1}^{\left[r_{1}\right]}, \ldots, a_{k}^{\left[r_{k}\right]}\right)} f(z) \omega_{a, r, p}^{|r|-1}=\int_{z \in \mathbb{C}^{|r|}, \varphi_{a, r, p}(z)<0} f(z)\left(d d^{c} \varphi_{a, r, p}\right)^{|r|-1} \wedge d d^{c} e^{\varphi_{a, r, p}} .
$$


Now, a straightforward calculation on $\mathbb{C}^{|r|}$ gives

$$
\begin{aligned}
\left(d d^{c} e^{p \varphi_{a, r, p}}\right)^{|r|} & =\left(d d^{c} \sum_{1 \leqslant s \leqslant k}\left|z_{s}\right|^{2 p / a_{s}}\right)^{|r|} \\
& =\prod_{1 \leqslant s \leqslant k}\left(\frac{p}{a_{s}}\right)^{r_{s}+1}\left|z_{s}\right|^{2 r_{s}\left(p / a_{s}-1\right)}\left(d d^{c}|z|^{2}\right)^{|r|} .
\end{aligned}
$$

On the other hand, we have $\left(d d^{c}|z|^{2}\right)^{|r|}=\frac{|r| !}{r_{1} ! \ldots r_{k} !} \prod_{1 \leqslant s \leqslant k}\left(d d^{c}\left|z_{s}\right|^{2}\right)^{r_{s}}$ and

$$
\begin{aligned}
\left(d d^{c} e^{p \varphi_{a, r, p}}\right)^{|r|} & =\left(p e^{p \varphi_{a, r, p}}\left(d d^{c} \varphi_{a, r, p}+p d \varphi_{a, r, p} \wedge d^{c} \varphi_{a, r, p}\right)\right)^{|r|} \\
& =|r| p^{|r|+1} e^{|r| p \varphi_{a, r, p}}\left(d d^{c} \varphi_{a, r, p}\right)^{|r|-1} \wedge d \varphi_{a, r, p} \wedge d^{c} \varphi_{a, r, p} \\
& =|r| p^{|r|+1} e^{(|r| p-1) \varphi_{a, r, p}}\left(d d^{c} \varphi_{a, r, p}\right)^{|r|-1} \wedge d d^{c} e^{\varphi_{a, r, p}},
\end{aligned}
$$

thanks to the homogeneity relation $\left(d d^{c} \varphi_{a, r, p}\right)^{|r|}=0$. Putting everything together, we find

$$
I_{p}=\int_{z \in \mathbb{C}^{|r|}, \varphi_{a, r, p}(z)<0} \frac{(|r|-1) ! p^{k-1} f(z)}{\left(\sum_{s}\left|z_{s}\right|^{2 p / a_{s}}\right)^{|r|-1 / p}} \prod_{s} \frac{\left(d d^{c}\left|z_{s}\right|^{2}\right)^{r_{s}}}{r_{s} ! a_{s}^{r_{s}+1}\left|z_{s}\right|^{2 r_{s}\left(1-p / a_{s}\right)}} .
$$

A standard calculation in polar coordinates with $z_{s}=\rho_{s} u_{s}, u_{s} \in S^{2 r_{s}-1}$, yields

$$
\frac{\left(d d^{c}\left|z_{s}\right|^{2}\right)^{r_{s}}}{\left|z_{s}\right|^{2 r_{s}}}=2 r_{s} \frac{d \rho_{s}}{\rho_{s}} d \mu_{s}\left(u_{s}\right)
$$

where $\mu_{s}$ is the $U\left(r_{s}\right)$-invariant probability measure on $S^{2 r_{s}-1}$. Therefore

$$
\begin{aligned}
I_{p} & =\int_{\varphi_{a, r, p}(z)<0} \frac{(|r|-1) ! p^{k-1} f\left(\rho_{1} u_{1}, \ldots, \rho_{k} u_{k}\right)}{\left(\sum_{1 \leqslant s \leqslant k} \rho_{s}^{2 p / a_{s}}\right)^{|r|-1 / p}} \prod_{s} \frac{2 \rho_{s}^{2 p r_{s} / a_{s}} \frac{d \rho_{s}}{\rho_{s}} d \mu_{s}\left(u_{s}\right)}{\left(r_{s}-1\right) ! a_{s}^{r_{s}+1}} \\
& =\int_{u_{s} \in S^{2 r_{s}-1}, \sum t_{s}<1} \frac{(|r|-1) ! p^{-1} f\left(t_{1}^{a_{1} / 2 p} u_{1}, \ldots, t_{k}^{a_{k} / 2 p} u_{k}\right)}{\left(\sum_{1 \leqslant s \leqslant k} t_{s}\right)^{|r|-1 / p}} \prod_{s} \frac{t_{s}^{r_{s}-1} d t_{s} d \mu_{s}\left(u_{s}\right)}{\left(r_{s}-1\right) ! a_{s}^{r_{s}}}
\end{aligned}
$$

by putting $t_{s}=\left|z_{s}\right|^{2 p / a_{s}}=\rho_{s}^{2 p / a_{s}}$, i.e. $\left.\left.\rho_{s}=t_{s}^{a_{s} / 2 p}, t_{s} \in\right] 0,1\right]$. We use still another change of variable $t_{s}=t x_{s}$ with $t=\sum_{1 \leqslant s \leqslant k} t_{s}$ and $\left.\left.x_{s} \in\right] 0,1\right], \sum_{1 \leqslant s \leqslant k} x_{s}=1$. Then

$$
d t_{1} \wedge \ldots \wedge d t_{k}=t^{k-1} d x d t \quad \text { where } d x=d x_{1} \wedge \ldots \wedge d x_{k-1}
$$

The $\mathbb{C}^{*}$ invariance of $f$ shows that

$$
\begin{aligned}
I_{p} & =\int_{\substack{\left.\left.u_{s} \in S^{2 r_{s}-1} \\
\Sigma x_{s}=1, t \in\right] 0,1\right]}}(|r|-1) ! f\left(x_{1}^{a_{s} / 2 p} u_{1}, \ldots, x_{k}^{a_{k} / 2 p} u_{k}\right) \prod_{1 \leqslant s \leqslant k} \frac{x_{s}^{r_{s}-1} d \mu_{s}\left(u_{s}\right)}{\left(r_{s}-1\right) ! a_{s}^{r_{s}}} \frac{d x d t}{p t^{1-1 / p}} \\
& =\int_{\substack{u_{s} \in S^{2 r_{s}-1} \\
\sum_{x_{s}=1}}}(|r|-1) ! f\left(x_{1}^{a_{s} / 2 p} u_{1}, \ldots, x_{k}^{a_{k} / 2 p} u_{k}\right) \prod_{1 \leqslant s \leqslant k} \frac{x_{s}^{r_{s}-1} d \mu_{s}\left(u_{s}\right)}{\left(r_{s}-1\right) ! a_{s}^{r_{s}}} d x .
\end{aligned}
$$


This is equivalent to the formula given in Proposition 1.13. We have $x_{s}^{2 a_{s} / p} \rightarrow 1$ as $p \rightarrow+\infty$, and by Lebesgue's bounded convergence theorem and Fubini's formula, we get

$$
\lim _{p \rightarrow+\infty} I_{p}=\frac{(|r|-1) !}{\prod_{s} a_{s}^{r_{s}}} \int_{(x, u) \in \Delta_{k-1} \times \prod S^{2 r_{s}-1}} f(u) \prod_{1 \leqslant s \leqslant k} \frac{x_{s}^{r_{s}-1}}{\left(r_{s}-1\right) !} d x d \mu(u) .
$$

It can be checked by elementary integrations by parts and induction on $k, r_{1}, \ldots, r_{k}$ that

$$
\int_{x \in \Delta_{k-1}} \prod_{1 \leqslant s \leqslant k} x_{s}^{r_{s}-1} d x_{1} \ldots d x_{k-1}=\frac{1}{(|r|-1) !} \prod_{1 \leqslant s \leqslant k}\left(r_{s}-1\right) ! .
$$

This implies that $(|r|-1) ! \prod_{1 \leqslant s \leqslant k} \frac{x_{s}^{r_{s}-1}}{\left(r_{s}-1\right) !} d x$ is a probability measure on $\Delta_{k-1}$ and that

$$
\lim _{p \rightarrow+\infty} I_{p}=\frac{1}{\prod_{s} a_{s}^{r_{s}}} \int_{u \in \prod S^{2 r_{s}-1}} f(u) d \mu(u) .
$$

Even without an explicit check, Formula (1.14) also follows from the fact that we must have equality for $f(z) \equiv 1$ in the latter equality, if we take into account the volume formula (1.11).

\section{Probabilistic estimate of the curvature of $k$-jet bundles}

Let $(X, V)$ be a compact complex directed non singular variety. To avoid any technical difficulty at this point, we first assume that $V$ is a holomorphic vector subbundle of $T_{X}$, equipped with a smooth Hermitian metric $h$.

According to the notation already specified in the introduction, we denote by $J^{k} V$ the bundle of $k$-jets of holomorphic curves $f:(\mathbb{C}, 0) \rightarrow X$ tangent to $V$ at each point. Let us set $n=\operatorname{dim}_{\mathbb{C}} X$ and $r=\operatorname{rank}_{\mathbb{C}} V$. Then $J^{k} V \rightarrow X$ is an algebraic fiber bundle with typical fiber $\mathbb{C}^{r k}$ (see below). It has a canonical $\mathbb{C}^{*}$-action defined by $\lambda \cdot f:(\mathbb{C}, 0) \rightarrow X$, $(\lambda \cdot f)(t)=f(\lambda t)$. Fix a point $x_{0}$ in $X$ and a local holomorphic coordinate system $\left(z_{1}, \ldots, z_{n}\right)$ centered at $x_{0}$ such that $V_{x_{0}}$ is the vector subspace $\left\langle\partial / \partial z_{1}, \ldots, \partial / \partial z_{r}\right\rangle$ at $x_{0}$. Then, in a neighborhood $U$ of $x_{0}, V$ admits a holomorphic frame of the form

$$
\frac{\partial}{\partial z_{\beta}}+\sum_{r+1 \leqslant \alpha \leqslant n} a_{\alpha \beta}(z) \frac{\partial}{\partial z_{\alpha}}, \quad 1 \leqslant \beta \leqslant r, \quad a_{\alpha \beta}(0)=0 .
$$

Let $f(t)=\left(f_{1}(t), \ldots, f_{n}(t)\right)$ be a $k$-jet of curve tangent to $V$ starting from a point $f(0)=$ $x \in U$. Such a curve is entirely determined by its initial point and by the projection $\widetilde{f}(t):=\left(f_{1}(t), \ldots, f_{r}(t)\right)$ to the first $r$-components, since the condition $f^{\prime}(t) \in V_{f(t)}$ implies that the other components must satisfy the ordinary differential equation

$$
f_{\alpha}^{\prime}(t)=\sum_{1 \leqslant \beta \leqslant r} a_{\alpha \beta}(f(t)) f_{\beta}^{\prime}(t)
$$

This implies that the $k$-jet of $f$ is entirely determined by the initial point $x$ and the Taylor expansion

$$
\widetilde{f}(t)-\widetilde{x}=\xi_{1} t+\xi_{2} t^{2}+\ldots+\xi_{k} t^{k}+O\left(t^{k+1}\right)
$$


where $\xi_{s}=\left(\xi_{s \alpha}\right)_{1 \leqslant \alpha \leqslant r} \in \mathbb{C}^{r}$. The $\mathbb{C}^{*}$ action $(\lambda, f) \mapsto \lambda \cdot f$ is then expressed in coordinates by the weighted action

$$
\lambda \cdot\left(\xi_{1}, \xi_{2}, \ldots, \xi_{k}\right)=\left(\lambda \xi_{1}, \lambda^{2} \xi_{2}, \ldots, \lambda^{k} \xi_{k}\right)
$$

associated with the weight $a=\left(1^{[r]}, 2^{[r]}, \ldots, k^{[r]}\right)$. The quotient projectivized $k$-jet bundle

$$
X_{k}^{\mathrm{GG}}:=\left(J^{k} V \backslash\{0\}\right) / \mathbb{C}^{*}
$$

considered by Green and Griffiths [GG79] is therefore in a natural way a $P\left(1^{[r]}, 2^{[r]}, \ldots, k^{[r]}\right)$ weighted projective bundle over $X$. As such, it possesses a canonical sheaf $\mathscr{\sigma}_{X_{k}^{\mathrm{GG}}}(1)$ such that $\mathscr{\sigma}_{X_{k}^{\mathrm{GG}}}(m)$ is invertible when $m$ is a multiple of $\operatorname{lcm}(1,2, \ldots, k)$. Under the natural projection $\pi_{k}: X_{k}^{\mathrm{GG}} \rightarrow X$, the direct image $\left(\pi_{k}\right)_{*} \mathscr{O}_{X_{k}^{\mathrm{GG}}}(m)$ coincides with the sheaf of sections of the bundle $E_{k, m}^{\mathrm{GG}} V^{*}$ of jet differentials of order $k$ and degree $m$, namely polynomials

$$
P\left(z ; \xi_{1}, \ldots, \xi_{k}\right)=\sum_{\alpha_{\ell} \in \mathbb{N}^{r}, 1 \leqslant \ell \leqslant k} a_{\alpha_{1} \ldots \alpha_{k}}(z) \xi_{1}^{\alpha_{1}} \ldots \xi_{k}^{\alpha_{k}}
$$

of weighted degree $\left|\alpha_{1}\right|+2\left|\alpha_{2}\right|+\ldots+k\left|\alpha_{k}\right|=m$ on $J^{k} V$ with holomorphic coefficients. The jet differentials operate on germs of curves as differential operators

$$
P(f)(t)=\sum a_{\alpha_{1} \ldots \alpha_{k}}(f(t)) f^{\prime}(t)^{\alpha_{1}} \ldots f^{(k)}(t)^{\alpha_{k}} .
$$

In the sequel, we do not make any further use of coordinate frames as (2.1), because they need not be related in any way to the Hermitian metric $h$ of $V$. Instead, we choose a local holomorphic coordinate frame $\left(e_{\alpha}(z)\right)_{1 \leqslant \alpha \leqslant r}$ of $V$ on a neighborhood $U$ of $x_{0}$, such that

$$
\left\langle e_{\alpha}(z), e_{\beta}(z)\right\rangle=\delta_{\alpha \beta}+\sum_{1 \leqslant i, j \leqslant n, 1 \leqslant \alpha, \beta \leqslant r} c_{i j \alpha \beta} z_{i} \bar{z}_{j}+O\left(|z|^{3}\right)
$$

for suitable complex coefficients $\left(c_{i j \alpha \beta}\right)$. It is a standard fact that such a normalized coordinate system always exists, and that the Chern curvature tensor $\frac{i}{2 \pi} D_{V, h}^{2}$ of $(V, h)$ at $x_{0}$ is then given by

$$
\Theta_{V, h}\left(x_{0}\right)=-\frac{i}{2 \pi} \sum_{i, j, \alpha, \beta} c_{i j \alpha \beta} d z_{i} \wedge d \bar{z}_{j} \otimes e_{\alpha}^{*} \otimes e_{\beta} .
$$

Also, instead of defining the vectors $\xi_{s} \in \mathbb{C}^{r}$ as in (2.2), we consider a local holomorphic connection $\nabla$ on $V_{\mid U}$ (e.g. the one which turns $\left(e_{\alpha}\right)$ into a parallel frame), and take $\xi_{k}=\nabla^{k} f(0) \in V_{x}$ defined inductively by $\nabla^{1} f=f^{\prime}$ and $\nabla^{s} f=\nabla_{f^{\prime}}\left(\nabla^{s-1} f\right)$. This is just another way of parametrizing the fibers of $J^{k} V$ over $U$ by the vector bundle $V_{\mid U}^{k}$. Notice that this is highly dependent on $\nabla$ (the bundle $J^{k} V$ actually does not carry a vector bundle or even affine bundle structure); however, the expression of the weighted action (2.3) is unchanged in this new setting. Now, we fix a finite open covering $\left(U_{\alpha}\right)_{\alpha \in I}$ of $X$ by open coordinate charts such that $V_{\mid U_{\alpha}}$ is trivial, along with holomorphic connections $\nabla_{\alpha}$ on $V_{\mid U_{\alpha}}$. Let $\theta_{\alpha}$ be a partition of unity of $X$ subordinate to the covering $\left(U_{\alpha}\right)$. Let 
us fix $p>0$ and small parameters $1=\varepsilon_{1} \gg \varepsilon_{2} \gg \ldots \gg \varepsilon_{k}>0$. Then we define a global weighted exhaustion on $J^{k} V$ by putting for any $k$-jet $f \in J_{x}^{k} V$

$$
\Psi_{h, p, \varepsilon}(f):=\left(\sum_{\alpha \in I} \theta_{\alpha}(x) \sum_{1 \leqslant s \leqslant k} \varepsilon_{s}^{2 p}\left\|\nabla_{\alpha}^{s} f(0)\right\|_{h(x)}^{2 p / s}\right)^{1 / p}
$$

where \|\|$_{h(x)}$ is the Hermitian metric $h$ of $V$ evaluated on the fiber $V_{x}, x=f(0)$. The function $\Psi_{h, p, \varepsilon}$ satisfies the fundamental homogeneity property

$$
\Psi_{h, p, \varepsilon}(\lambda \cdot f)=\Psi_{h, p, \varepsilon}(f)|\lambda|^{2}
$$

with respect to the $\mathbb{C}^{*}$ action on $J^{k} V$, in other words, it induces a Hermitian metric on the dual $L^{*}$ of the tautological $\mathbb{Q}$-line bundle $L_{k}=\mathscr{G}_{X_{k}^{\mathrm{GG}}}(1)$ over $X_{k}^{\mathrm{GG}}$. The curvature of $L_{k}$ is given by

$$
\pi_{k}^{*} \Theta_{L_{k}, \Psi_{h, p, \varepsilon}^{*}}=d d^{c} \log \Psi_{h, p, \varepsilon}
$$

where $\pi_{k}: J^{k} V \backslash\{0\} \rightarrow X_{k}^{\mathrm{GG}}$ is the canonical projection. Our next goal is to compute precisely the curvature and to apply holomorphic Morse inequalities to $L \rightarrow X_{k}^{\mathrm{GG}}$ with the above metric. It might look a priori like an untractable problem, since the definition of $\Psi_{h, p, \varepsilon}$ is a rather unnatural one. However, the "miracle" is that the asymptotic behavior of $\Psi_{h, p, \varepsilon}$ as $\varepsilon_{s} / \varepsilon_{s-1} \rightarrow 0$ is in some sense uniquely defined and very natural. It will lead to a computable asymptotic formula, which is moreover simple enough to produce useful results.

(2.12) Lemma. On each coordinate chart $U$ equipped with a holomorphic connection $\nabla$ of $V_{\mid U}$, let us define the components of a $k$-jet $f \in J^{k} V$ by $\xi_{s}=\nabla^{s} f(0)$, and consider the rescaling transformation

$$
\rho_{\nabla, \varepsilon}\left(\xi_{1}, \xi_{2}, \ldots, \xi_{k}\right)=\left(\varepsilon_{1}^{1} \xi_{1}, \varepsilon_{2}^{2} \xi_{2}, \ldots, \varepsilon_{k}^{k} \xi_{k}\right) \quad \text { on } J_{x}^{k} V, x \in U
$$

(it commutes with the $\mathbb{C}^{*}$-action but is otherwise unrelated and not canonically defined over $X$ as it depends on the choice of $\nabla)$. Then, if $p$ is a multiple of $\operatorname{lcm}(1,2, \ldots, k)$ and $\varepsilon_{s} / \varepsilon_{s-1} \rightarrow 0$ for all $s=2, \ldots, k$, the rescaled function $\Psi_{h, p, \varepsilon} \circ \rho_{\nabla, \varepsilon}^{-1}\left(\xi_{1}, \ldots, \xi_{k}\right)$ converges towards

$$
\left(\sum_{1 \leqslant s \leqslant k}\left\|\xi_{s}\right\|_{h}^{2 p / s}\right)^{1 / p}
$$

on every compact subset of $J^{k} V_{\mid U} \backslash\{0\}$, uniformly in $C^{\infty}$ topology.

Proof. Let $U \subset X$ be an open set on which $V_{\mid U}$ is trivial and equipped with some holomorphic connection $\nabla$. Let us pick another holomorphic connection $\widetilde{\nabla}=\nabla+\Gamma$ where $\Gamma \in H^{0}\left(U, \Omega_{X}^{1} \otimes \operatorname{Hom}(V, V)\right.$. Then $\widetilde{\nabla}^{2} f=\nabla^{2} f+\Gamma(f)\left(f^{\prime}\right) \cdot f^{\prime}$, and inductively we get

$$
\widetilde{\nabla}^{s} f=\nabla^{s} f+P_{s}\left(f ; \nabla^{1} f, \ldots, \nabla^{s-1} f\right)
$$

where $P\left(x ; \xi_{1}, \ldots, \xi_{s-1}\right)$ is a polynomial with holomorphic coefficients in $x \in U$ which is of weighted homogeneous degree $s$ in $\left(\xi_{1}, \ldots, \xi_{s-1}\right)$. In other words, the corresponding change in the parametrization of $J^{k} V_{\mid U}$ is given by a $\mathbb{C}^{*}$-homogeneous transformation

$$
\widetilde{\xi}_{s}=\xi_{s}+P_{s}\left(x ; \xi_{1}, \ldots, \xi_{s-1}\right)
$$


Let us introduce the corresponding rescaled components

$$
\left(\xi_{1, \varepsilon}, \ldots, \xi_{k, \varepsilon}\right)=\left(\varepsilon_{1}^{1} \xi_{1}, \ldots, \varepsilon_{k}^{k} \xi_{k}\right), \quad\left(\widetilde{\xi}_{1, \varepsilon}, \ldots, \widetilde{\xi}_{k, \varepsilon}\right)=\left(\varepsilon_{1}^{1} \widetilde{\xi}_{1}, \ldots, \varepsilon_{k}^{k} \widetilde{\xi}_{k}\right)
$$

Then

$$
\begin{aligned}
\widetilde{\xi}_{s, \varepsilon} & =\xi_{s, \varepsilon}+\varepsilon_{s}^{s} P_{s}\left(x ; \varepsilon_{1}^{-1} \xi_{1, \varepsilon}, \ldots, \varepsilon_{s-1}^{-(s-1)} \xi_{s-1, \varepsilon}\right) \\
& =\xi_{s, \varepsilon}+O\left(\varepsilon_{s} / \varepsilon_{s-1}\right)^{s} O\left(\left\|\xi_{1, \varepsilon}\right\|+\ldots+\left\|\xi_{s-1, \varepsilon}\right\|^{1 /(s-1)}\right)^{s}
\end{aligned}
$$

and the error terms are thus polynomials of fixed degree with arbitrarily small coefficients as $\varepsilon_{s} / \varepsilon_{s-1} \rightarrow 0$. Now, the definition of $\Psi_{h, p, \varepsilon}$ consists of glueing the sums

$$
\sum_{1 \leqslant s \leqslant k} \varepsilon_{s}^{2 p}\left\|\xi_{k}\right\|_{h}^{2 p / s}=\sum_{1 \leqslant s \leqslant k}\left\|\xi_{k, \varepsilon}\right\|_{h}^{2 p / s}
$$

corresponding to $\xi_{k}=\nabla_{\alpha}^{s} f(0)$ by means of the partition of unity $\sum \theta_{\alpha}(x)=1$. We see that by using the rescaled variables $\xi_{s, \varepsilon}$ the changes occurring when replacing a connection $\nabla_{\alpha}$ by an alternative one $\nabla_{\beta}$ are arbitrary small in $C^{\infty}$ topology, with error terms uniformly controlled in terms of the ratios $\varepsilon_{s} / \varepsilon_{s-1}$ on all compact subsets of $V^{k} \backslash\{0\}$. This shows that in $C^{\infty}$ topology, $\Psi_{h, p, \varepsilon} \circ \rho_{\nabla, \varepsilon}^{-1}\left(\xi_{1}, \ldots, \xi_{k}\right)$ converges uniformly towards $\left(\sum_{1 \leqslant s \leqslant k}\left\|\xi_{k}\right\|_{h}^{2 p / s}\right)^{1 / p}$, whatever the trivializing open set $U$ and the holomorphic connection $\nabla$ used to evaluate the components and perform the rescaling are.

Now, we fix a point $x_{0} \in X$ and a local holomorphic frame $\left(e_{\alpha}(z)\right)_{1 \leqslant \alpha \leqslant r}$ satisfying (2.7) on a neighborhood $U$ of $x_{0}$. We introduce the rescaled components $\xi_{s}=\varepsilon_{s}^{s} \nabla^{s} f(0)$ on $J^{k} V_{\mid U}$ and compute the curvature of

$$
\Psi_{h, p, \varepsilon} \circ \rho_{\nabla, \varepsilon}^{-1}\left(z ; \xi_{1}, \ldots, \xi_{k}\right) \simeq\left(\sum_{1 \leqslant s \leqslant k}\left\|\xi_{s}\right\|_{h}^{2 p / s}\right)^{1 / p}
$$

(by Lemma 2.12, the errors can be taken arbitrary small in $C^{\infty}$ topology). We write $\xi_{s}=\sum_{1 \leqslant \alpha \leqslant r} \xi_{s \alpha} e_{\alpha}$. By $(2.7)$ we have

$$
\left\|\xi_{s}\right\|_{h}^{2}=\sum_{\alpha}\left|\xi_{s \alpha}\right|^{2}+\sum_{i, j, \alpha, \beta} c_{i j \alpha \beta} z_{i} \bar{z}_{j} \xi_{s \alpha} \bar{\xi}_{s \beta}+O\left(|z|^{3}|\xi|^{2}\right) .
$$

The question is to evaluate the curvature of the weighted metric defined by

$$
\begin{aligned}
\Psi\left(z ; \xi_{1}, \ldots, \xi_{k}\right) & =\left(\sum_{1 \leqslant s \leqslant k}\left\|\xi_{s}\right\|_{h}^{2 p / s}\right)^{1 / p} \\
& =\left(\sum_{1 \leqslant s \leqslant k}\left(\sum_{\alpha}\left|\xi_{s \alpha}\right|^{2}+\sum_{i, j, \alpha, \beta} c_{i j \alpha \beta} z_{i} \bar{z}_{j} \xi_{s \alpha} \bar{\xi}_{s \beta}\right)^{p / s}\right)^{1 / p}+O\left(|z|^{3}\right) .
\end{aligned}
$$

We set $\left|\xi_{s}\right|^{2}=\sum_{\alpha}\left|\xi_{s \alpha}\right|^{2}$. A straightforward calculation yields

$$
\begin{aligned}
& \log \Psi\left(z ; \xi_{1}, \ldots, \xi_{k}\right)= \\
& =\frac{1}{p} \log \sum_{1 \leqslant s \leqslant k}\left|\xi_{s}\right|^{2 p / s}+\sum_{1 \leqslant s \leqslant k} \frac{1}{s} \frac{\left|\xi_{s}\right|^{2 p / s}}{\sum_{t}\left|\xi_{t}\right|^{2 p / t}} \sum_{i, j, \alpha, \beta} c_{i j \alpha \beta} z_{i} \bar{z}_{j} \frac{\xi_{s \alpha} \bar{\xi}_{s \beta}}{\left|\xi_{s}\right|^{2}}+O\left(|z|^{3}\right) .
\end{aligned}
$$


By (2.11), the curvature form of $L_{k}=\mathscr{O}_{X_{k}^{G G}}(1)$ is given at the central point $x_{0}$ by the following formula.

(2.13) Proposition. With the above choice of coordinates and with respect to the rescaled components $\xi_{s}=\varepsilon_{s}^{s} \nabla^{s} f(0)$ at $x_{0} \in X$, we have the approximate expression

$$
\Theta_{L_{k}, \Psi_{h, p, \varepsilon}^{*}}\left(x_{0},[\xi]\right) \simeq \omega_{a, r, p}(\xi)+\frac{i}{2 \pi} \sum_{1 \leqslant s \leqslant k} \frac{1}{s} \frac{\left|\xi_{s}\right|^{2 p / s}}{\sum_{t}\left|\xi_{t}\right|^{2 p / t}} \sum_{i, j, \alpha, \beta} c_{i j \alpha \beta} \frac{\xi_{s \alpha} \bar{\xi}_{s \beta}}{\left|\xi_{s}\right|^{2}} d z_{i} \wedge d \bar{z}_{j}
$$

where the error terms are $O\left(\max _{2 \leqslant s \leqslant k}\left(\varepsilon_{s} / \varepsilon_{s-1}\right)^{s}\right)$ uniformly on the compact variety $X_{k}^{\mathrm{GG}}$. Here $\omega_{a, r, p}$ is the (degenerate) Kähler metric associated with the weight $a=$ $\left(1^{[r]}, 2^{[r]}, \ldots, k^{[r]}\right)$ of the canonical $\mathbb{C}^{*}$ action on $J^{k} V$.

Thanks to the uniform approximation, we can (and will) neglect the error terms in the calculations below. Since $\omega_{a, r, p}$ is positive definite on the fibers of $X_{k}^{\mathrm{GG}} \rightarrow X$ (at least outside of the axes $\left.\xi_{s}=0\right)$, the index of the $(1,1)$ curvature form $\Theta_{L_{k}, \Psi_{h, p, \varepsilon}^{*}}(z,[\xi])$ is equal to the index of the $(1,1)$-form

$$
\gamma_{k}(z, \xi):=\frac{i}{2 \pi} \sum_{1 \leqslant s \leqslant k} \frac{1}{s} \frac{\left|\xi_{s}\right|^{2 p / s}}{\sum_{t}\left|\xi_{t}\right|^{2 p / t}} \sum_{i, j, \alpha, \beta} c_{i j \alpha \beta}(z) \frac{\xi_{s \alpha} \bar{\xi}_{s \beta}}{\left|\xi_{s}\right|^{2}} d z_{i} \wedge d \bar{z}_{j}
$$

depending only on the differentials $\left(d z_{j}\right)_{1 \leqslant j \leqslant n}$ on $X$. The $q$-index integral of $\left(L_{k}, \Psi_{h, p, \varepsilon}^{*}\right)$ on $X_{k}^{\mathrm{GG}}$ is therefore equal to

$$
\begin{aligned}
& \int_{X_{k}^{\mathrm{GG}}\left(L_{k}, q\right)} \Theta_{L_{k}, \Psi_{h, p, \varepsilon}^{*}+k r-1}^{*}= \\
& =\frac{(n+k r-1) !}{n !(k r-1) !} \int_{z \in X} \int_{\xi \in P(1[r], \ldots, k[r])} \omega_{a, r, p}^{k r-1}(\xi) \mathbb{1}_{\gamma_{k}, q}(z, \xi) \gamma_{k}(z, \xi)^{n}
\end{aligned}
$$

where $\mathbb{1}_{\gamma_{k}, q}(z, \xi)$ is the characteristic function of the open set of points where $\gamma_{k}(z, \xi)$ has signature $(n-q, q)$ in terms of the $d z_{j}$ 's. Notice that since $\gamma_{k}(z, \xi)^{n}$ is a determinant, the product $\mathbb{1}_{\gamma_{k}, q}(z, \xi) \gamma_{k}(z, \xi)^{n}$ gives rise to a continuous function on $X_{k}^{\mathrm{GG}}$. Formula 1.14 with $r_{1}=\ldots=r_{k}=r$ and $a_{s}=s$ yields the slightly more explicit integral

$$
\begin{aligned}
& \int_{X_{k}^{\mathrm{GG}}\left(L_{k}, q\right)} \Theta_{L_{k}, \Psi_{h, p, \varepsilon}^{*}}^{n+k r-1}=\frac{(n+k r-1) !}{n !(k !)^{r}} \times \\
& \int_{z \in X} \int_{(x, u) \in \Delta_{k-1} \times\left(S^{2 r-1}\right)^{k}} \mathbb{1}_{g_{k}, q}(z, x, u) g_{k}(z, x, u)^{n} \frac{\left(x_{1} \ldots x_{k}\right)^{r-1}}{(r-1) !^{k}} d x d \mu(u),
\end{aligned}
$$

where $g_{k}(z, x, u)=\gamma_{k}\left(z, x_{1}^{1 / 2 p} u_{1}, \ldots, x_{k}^{k / 2 p} u_{k}\right)$ is given by

$$
g_{k}(z, x, u)=\frac{i}{2 \pi} \sum_{1 \leqslant s \leqslant k} \frac{1}{s} x_{s} \sum_{i, j, \alpha, \beta} c_{i j \alpha \beta}(z) u_{s \alpha} \bar{u}_{s \beta} d z_{i} \wedge d \bar{z}_{j}
$$

and $\mathbb{1}_{g_{k}, q}(z, x, u)$ is the characteristic function of its $q$-index set. Here

$$
d \nu_{k, r}(x)=(k r-1) ! \frac{\left(x_{1} \ldots x_{k}\right)^{r-1}}{(r-1) !^{k}} d x
$$


is a probability measure on $\Delta_{k-1}$, and we can rewrite

$$
\begin{aligned}
& \int_{X_{k}^{\mathrm{GG}}\left(L_{k}, q\right)} \Theta_{L_{k}, \Psi_{h, p, \varepsilon}^{*}}^{n+k r-1}=\frac{(n+k r-1) !}{n !(k !)^{r}(k r-1) !} \times \\
& \int_{z \in X} \int_{(x, u) \in \Delta_{k-1} \times\left(S^{2 r-1}\right)^{k}} \mathbb{1}_{g_{k}, q}(z, x, u) g_{k}(z, x, u)^{n} d \nu_{k, r}(x) d \mu(u) .
\end{aligned}
$$

Now, formula (2.15) shows that $g_{k}(z, x, u)$ is a "Monte Carlo" evaluation of the curvature tensor, obtained by averaging the curvature at random points $u_{s} \in S^{2 r-1}$ with certain positive weights $x_{s} / s$; we should then think of the $k$-jet $f$ as some sort of random parameter such that the derivatives $\nabla^{k} f(0)$ are uniformly distributed in all directions. Let us compute the expected value of $(x, u) \mapsto g_{k}(z, x, u)$ with respect to the probability measure $d \nu_{k, r}(x) d \mu(u)$. Since $\int_{S^{2 r-1}} u_{s \alpha} \bar{u}_{s \beta} d \mu\left(u_{s}\right)=\frac{1}{r} \delta_{\alpha \beta}$ and $\int_{\Delta_{k-1}} x_{s} d \nu_{k, r}(x)=\frac{1}{k}$, we find

$$
\mathbf{E}\left(g_{k}(z, \bullet, \bullet)\right)=\frac{1}{k r} \sum_{1 \leqslant s \leqslant k} \frac{1}{s} \cdot \frac{i}{2 \pi} \sum_{i, j, \alpha} c_{i j \alpha \alpha}(z) d z_{i} \wedge d \bar{z}_{j} .
$$

In other words, we get the normalized trace of the curvature, i.e.

$$
\mathbf{E}\left(g_{k}(z, \bullet, \bullet)\right)=\frac{1}{k r}\left(1+\frac{1}{2}+\ldots+\frac{1}{k}\right) \Theta_{\operatorname{det}\left(V^{*}\right), \operatorname{det} h^{*}}
$$

where $\Theta_{\operatorname{det}\left(V^{*}\right), \operatorname{det} h^{*}}$ is the $(1,1)$-curvature form of $\operatorname{det}\left(V^{*}\right)$ with the metric induced by $h$. It is natural to guess that $g_{k}(z, x, u)$ behaves asymptotically as its expected value $\mathbf{E}\left(g_{k}(z, \bullet, \bullet)\right)$ when $k$ tends to infinity. If we replace brutally $g_{k}$ by its expected value in (2.17), we get the integral

$$
\frac{(n+k r-1) !}{n !(k !)^{r}(k r-1) !} \frac{1}{(k r)^{n}}\left(1+\frac{1}{2}+\ldots+\frac{1}{k}\right)^{n} \int_{X} \mathbb{1}_{\eta, q} \eta^{n}
$$

where $\eta:=\Theta_{\operatorname{det}\left(V^{*}\right), \operatorname{det} h^{*}}$ and $\mathbb{1}_{\eta, q}$ is the characteristic function of its $q$-index set in $X$. The leading constant is equivalent to $(\log k)^{n} / n !(k !)^{r}$ modulo a multiplicative factor $1+O(1 / \log k)$. By working out a more precise analysis of the deviation, we will prove the following result.

(2.19) Probabilistic estimate. Fix smooth Hermitian metrics $h$ on $V$ and $\omega=$ $\frac{i}{2 \pi} \sum \omega_{i j} d z_{i} \wedge d \bar{z}_{j}$ on $X$. Denote by $\Theta_{V, h}=-\frac{i}{2 \pi} \sum c_{i j \alpha \beta} d z_{i} \wedge d \bar{z}_{j} \otimes e_{\alpha}^{*} \otimes e_{\beta}$ the curvature tensor of $V$ with respect to an h-orthonormal frame $\left(e_{\alpha}\right)$, and put

$$
\eta(z)=\Theta_{\operatorname{det}\left(V^{*}\right), \operatorname{det} h^{*}}=\frac{i}{2 \pi} \sum_{1 \leqslant i, j \leqslant n} \eta_{i j} d z_{i} \wedge d \bar{z}_{j}, \quad \eta_{i j}=\sum_{1 \leqslant \alpha \leqslant r} c_{i j \alpha \alpha} .
$$

Finally consider the $k$-jet line bundle $L_{k}=\mathscr{O}_{X_{k}^{\mathrm{GG}}}(1) \rightarrow X_{k}^{\mathrm{GG}}$ equipped with the induced metric $\Psi_{h, p, \varepsilon}^{*}$ (as defined above, with $\left.1=\varepsilon_{1} \gg \varepsilon_{2} \gg \ldots \gg \varepsilon_{k}>0\right)$. When $k$ tends to infinity, the integral of the top power of the curvature of $L_{k}$ on its $q$-index set $X_{k}^{\mathrm{GG}}\left(L_{k}, q\right)$ is given by

$$
\int_{X_{k}^{\mathrm{GG}}\left(L_{k}, q\right)} \Theta_{L_{k}, \Psi_{h, p, \varepsilon}^{*}}^{n+k r-1}=\frac{(\log k)^{n}}{n !(k !)^{r}}\left(\int_{X} \mathbb{1}_{\eta, q} \eta^{n}+O\left((\log k)^{-1}\right)\right)
$$


for all $q=0,1, \ldots, n$, and the error term $O\left((\log k)^{-1}\right)$ can be bounded explicitly in terms of $\Theta_{V}, \eta$ and $\omega$. Moreover, the left hand side is identically zero for $q>n$.

The final statement follows from the observation that the curvature of $L_{k}$ is positive along the fibers of $X_{k}^{\mathrm{GG}} \rightarrow X$, by the plurisubharmonicity of the weight (this is true even when the partition of unity terms are taken into account, since they depend only on the base); therefore the $q$-index sets are empty for $q>n$. We start with three elementary lemmas.

(2.20) Lemma. The integral

$$
I_{k, r, n}=\int_{\Delta_{k-1}}\left(\sum_{1 \leqslant s \leqslant k} \frac{x_{s}}{s}\right)^{n} d \nu_{k, r}(x)
$$

is given by the expansion

$$
I_{k, r, n}=\sum_{1 \leqslant s_{1}, s_{2}, \ldots, s_{n} \leqslant k} \frac{1}{s_{1} s_{2} \ldots s_{n}} \frac{(k r-1) !}{(r-1) !^{k}} \frac{\prod_{1 \leqslant i \leqslant k}\left(r-1+\beta_{i}\right) !}{(k r+n-1) !} .
$$

where $\beta_{i}=\beta_{i}(s)=\operatorname{card}\left\{j ; s_{j}=i\right\}, \sum \beta_{i}=n, 1 \leqslant i \leqslant k$. The quotient

$$
I_{k, r, n} / \frac{r^{n}}{k r(k r+1) \ldots(k r+n-1)}\left(1+\frac{1}{2}+\ldots+\frac{1}{k}\right)^{n}
$$

is bounded below by 1 and bounded above by

$$
1+\frac{1}{3} \sum_{m=2}^{n} \frac{2^{m} n !}{(n-m) !}\left(1+\frac{1}{2}+\ldots+\frac{1}{k}\right)^{-m}=1+O\left((\log k)^{-2}\right)
$$

As a consequence

$$
\begin{aligned}
I_{k, r, n} & =\frac{1}{k^{n}}\left(\left(1+\frac{1}{2}+\ldots+\frac{1}{k}\right)^{n}+O\left((\log k)^{n-2}\right)\right) \\
& =\frac{(\log k+\gamma)^{n}+O\left((\log k)^{n-2}\right)}{k^{n}}
\end{aligned}
$$

where $\gamma$ is the Euler-Mascheroni constant.

Proof. Let us expand the $n$-th power $\left(\sum_{1 \leqslant s \leqslant k} \frac{x_{s}}{s}\right)^{n}$. This gives

$$
I_{k, r, n}=\sum_{1 \leqslant s_{1}, s_{2}, \ldots, s_{n} \leqslant k} \frac{1}{s_{1} s_{2} \ldots s_{n}} \int_{\Delta_{k-1}} x_{1}^{\beta_{1}} \ldots x_{k}^{\beta_{k}} d \nu_{k, r}(x)
$$

and by definition of the measure $\nu_{k, r}$ we have

$$
\int_{\Delta_{k-1}} x_{1}^{\beta_{1}} \ldots x_{k}^{\beta_{k}} d \nu_{k, r}(x)=\frac{(k r-1) !}{(r-1) !^{k}} \int_{\Delta_{k-1}} x_{1}^{r+\beta_{1}-1} \ldots x_{k}^{r+\beta_{k}-1} d x_{1} \ldots d x_{k} .
$$


By Formula (1.14), we find

$$
\begin{aligned}
\int_{\Delta_{k-1}} x_{1}^{\beta_{1}} \ldots x_{k}^{\beta_{k}} d \nu_{k, r}(x) & =\frac{(k r-1) !}{(r-1) ! k} \frac{\prod_{1 \leqslant i \leqslant k}\left(r+\beta_{i}-1\right) !}{(k r+n-1) !} \\
& =\frac{r^{n} \prod_{i, \beta_{i} \geqslant 1}\left(1+\frac{1}{r}\right)\left(1+\frac{2}{r}\right) \ldots\left(1+\frac{\beta_{i}-1}{r}\right)}{k r(k r+1) \ldots(k r+n-1)}
\end{aligned}
$$

and $(2.20 \mathrm{a})$ follows from the first equality. The final product is minimal when $r=1$, thus

$$
\begin{array}{rl}
\frac{r^{n}}{k r(k r+1) \ldots(k r+n-1)} \leqslant \int_{\Delta_{k-1}} x_{1}^{\beta_{1}} \ldots x_{k}^{\beta_{k}} & d \nu_{k, r}(x) \\
& \leqslant \frac{r^{n} \prod_{1 \leqslant i \leqslant k} \beta_{i} !}{k r(k r+1) \ldots(k r+n-1)}
\end{array}
$$

Also, the integral is maximal when all $\beta_{i}$ vanish except one, in which case one gets

$$
\int_{\Delta_{k-1}} x_{j}^{n} d \nu_{k, r}(x)=\frac{r(r+1) \ldots(r+n-1)}{k r(k r+1) \ldots(k r+n-1)} .
$$

By (2.21), we find the lower and upper bounds

$$
\begin{aligned}
& I_{k, r, n} \geqslant \frac{r^{n}}{k r(k r+1) \ldots(k r+n-1)}\left(1+\frac{1}{2}+\ldots+\frac{1}{k}\right)^{n}, \\
& I_{k, r, n} \leqslant \frac{r^{n}}{k r(k r+1) \ldots(k r+n-1)} \sum_{1 \leqslant s_{1}, \ldots, s_{n} \leqslant k} \frac{\beta_{1} ! \ldots \beta_{k} !}{s_{1} \ldots s_{n}} .
\end{aligned}
$$

In order to make the upper bound more explicit, we reorganize the $n$-tuple $\left(s_{1}, \ldots, s_{n}\right)$ into those indices $t_{1}<\ldots<t_{\ell}$ which appear a certain number of times $\alpha_{i}=\beta_{t_{i}} \geqslant 2$, and those, say $t_{\ell+1}<\ldots<t_{\ell+m}$, which appear only once. We have of course $\sum \beta_{i}=$ $n-m$, and each choice of the $t_{i}$ 's corresponds to $n ! / \alpha_{1} ! \ldots \alpha_{\ell}$ ! possibilities for the $n$-tuple $\left(s_{1}, \ldots, s_{n}\right)$. Therefore we get

$$
\sum_{1 \leqslant s_{1}, \ldots, s_{n} \leqslant k} \frac{\beta_{1} ! \ldots \beta_{k} !}{s_{1} \ldots s_{n}} \leqslant n ! \sum_{m=0}^{n} \sum_{\ell, \Sigma \alpha_{i}=n-m} \sum_{\left(t_{i}\right)} \frac{1}{t_{1}^{\alpha_{1}} \ldots t_{\ell}^{\alpha_{\ell}}} \frac{1}{t_{\ell+1} \ldots t_{\ell+m}} .
$$

A trivial comparison series vs. integral yields

$$
\sum_{s<t<+\infty} \frac{1}{t^{\alpha}} \leqslant \frac{1}{\alpha-1} \frac{1}{s^{\alpha-1}}
$$

and in this way, using successive integrations in $t_{\ell}, t_{\ell-1}, \ldots$, we get inductively

$$
\sum_{1 \leqslant t_{1}<\ldots<t_{\ell}<+\infty} \frac{1}{t_{1}^{\alpha_{1}} \ldots t_{\ell}^{\alpha_{\ell}}} \leqslant \frac{1}{\prod_{1 \leqslant i \leqslant \ell}\left(\alpha_{\ell-i+1}+\ldots+\alpha_{\ell}-i\right)} \leqslant \frac{1}{\ell !},
$$


since $\alpha_{i} \geqslant 2$ implies $\alpha_{\ell-i+1}+\ldots+\alpha_{\ell}-i \geqslant i$. On the other hand

$$
\sum_{1 \leqslant t_{\ell+1}<\ldots<t_{\ell+m} \leqslant k} \frac{1}{t_{\ell+1} \ldots t_{\ell+m}} \leqslant \frac{1}{m !} \sum_{1 \leqslant s_{1}, \ldots, s_{m} \leqslant k} \frac{1}{s_{1} \ldots s_{m}}=\frac{1}{m !}\left(1+\frac{1}{2}+\ldots+\frac{1}{k}\right)^{m} .
$$

Since partitions $\alpha_{1}+\ldots+\alpha_{\ell}=n-m$ satisfying the additional restriction $\alpha_{i} \geqslant 2$ correspond to $\alpha_{i}^{\prime}=\alpha_{i}-2$ satisfying $\sum \alpha_{i}^{\prime}=n-m-2 \ell$, their number is equal to

$$
\left(\begin{array}{c}
n-m-2 \ell+\ell-1 \\
\ell-1
\end{array}\right)=\left(\begin{array}{c}
n-m-\ell-1 \\
\ell-1
\end{array}\right) \leqslant 2^{n-m-\ell-1}
$$

and we infer from this

$$
\sum_{1 \leqslant s_{1}, \ldots, s_{n} \leqslant k} \frac{\beta_{1} ! \ldots \beta_{k} !}{s_{1} \ldots s_{n}} \leqslant \sum_{\substack{\ell \geqslant 1 \\ 2 \ell+m \leqslant n}} \frac{2^{n-m-\ell-1} n !}{\ell ! m !}\left(1+\frac{1}{2}+\ldots+\frac{1}{k}\right)^{m}+\left(1+\frac{1}{2}+\ldots+\frac{1}{k}\right)^{n}
$$

where the last term corresponds to the special case $\ell=0, m=n$. Therefore

$$
\begin{aligned}
\sum_{1 \leqslant s_{i} \leqslant k} \frac{\beta_{1} ! \ldots \beta_{k} !}{s_{1} \ldots s_{n}} & \leqslant \frac{e^{1 / 2}-1}{2} \sum_{m=0}^{n-2} \frac{2^{n-m} n !}{m !}\left(1+\frac{1}{2}+\ldots+\frac{1}{k}\right)^{m}+\left(1+\frac{1}{2}+\ldots+\frac{1}{k}\right)^{n} \\
& \leqslant \frac{1}{3} \sum_{m=2}^{n} \frac{2^{m} n !}{(n-m) !}\left(1+\frac{1}{2}+\ldots+\frac{1}{k}\right)^{n-m}+\left(1+\frac{1}{2}+\ldots+\frac{1}{k}\right)^{n} .
\end{aligned}
$$

This estimate combined with $(2.23,2.24)$ implies the upper bound $(2.20 \mathrm{~b})$ (the lower bound 1 being now obvious). The asymptotic estimate $(2.20 \mathrm{c})$ follows immediately.

(2.25) Lemma. If $A$ is a Hermitian $n \times n$ matrix, set $\mathbb{1}_{A, q}$ to be equal to 1 if $A$ has signature $(n-q, q)$ and 0 otherwise. Then for all $n \times n$ Hermitian matrices $A, B$ we have the estimate

$$
\left|\mathbb{1}_{A, q} \operatorname{det} A-\mathbb{1}_{B, q} \operatorname{det} B\right| \leqslant\|A-B\| \sum_{0 \leqslant i \leqslant n-1}\|A\|^{i}\|B\|^{n-1-i},
$$

where $\|A\|,\|B\|$ are the Hermitian operator norms of the matrices.

Proof. We first check that the estimate holds for $|\operatorname{det} A-\operatorname{det} B|$. Let $\lambda_{1} \leqslant \ldots \leqslant \lambda_{n}$ be the eigenvalues of $A$ and $\lambda_{1}^{\prime} \leqslant \ldots \leqslant \lambda_{n}^{\prime}$ be the eigenvalues of $B$. We have $\left|\lambda_{i}\right| \leqslant\|A\|$, $\left|\lambda_{i}^{\prime}\right| \leqslant\|B\|$ and the minimax principle implies that $\left|\lambda_{i}-\lambda_{i}^{\prime}\right| \leqslant\|A-B\|$. We then get the desired estimate by writing

$$
\operatorname{det} A-\operatorname{det} B=\lambda_{1} \ldots \lambda_{n}-\lambda_{1}^{\prime} \ldots \lambda_{n}^{\prime}=\sum_{1 \leqslant i \leqslant n} \lambda_{1} \ldots \lambda_{i-1}\left(\lambda_{i}-\lambda_{i}^{\prime}\right) \lambda_{i+1}^{\prime} \ldots \lambda_{n}^{\prime} .
$$

This already implies (2.25) if $A$ or $B$ is degenerate. If $A$ and $B$ are non degenerate we only have to prove the result when one of them (say $A$ ) has signature $(n-q, q)$ and the other one (say $B$ ) has a different signature. If we put $M(t)=(1-t) A+t B$, the already established estimate for the determinant yields

$$
\left|\frac{d}{d t} \operatorname{det} M(t)\right| \leqslant n\|A-B\|\|M(t)\| \leqslant n\|A-B\|((1-t)\|A\|+t\|B\|)^{n-1} .
$$


However, since the signature of $M(t)$ is not the same for $t=0$ and $t=1$, there must exist $\left.t_{0} \in\right] 0,1\left[\right.$ such that $\left(1-t_{0}\right) A+t_{0} B$ is degenerate. Our claim follows by integrating the differential estimate on the smallest such interval $\left[0, t_{0}\right]$, after observing that $M(0)=A$, $\operatorname{det} M\left(t_{0}\right)=0$, and that the integral of the right hand side on $[0,1]$ is the announced bound.

(2.26) Lemma. Let $Q_{A}$ be the Hermitian quadratic form associated with the Hermitian operator $A$ on $\mathbb{C}^{n}$. If $\mu$ is the rotation invariant probability measure on the unit sphere $S^{2 n-1}$ of $\mathbb{C}^{n}$ and $\lambda_{i}$ are the eigenvalues of $A$, we have

$$
\int_{|\zeta|=1}\left|Q_{A}(\zeta)\right|^{2} d \mu(\zeta)=\frac{1}{n(n+1)}\left(\sum \lambda_{i}^{2}+\left(\sum \lambda_{i}\right)^{2}\right)
$$

The norm $\|A\|=\max \left|\lambda_{i}\right|$ satisfies the estimate

$$
\frac{1}{n^{2}}\|A\|^{2} \leqslant \int_{|\zeta|=1}\left|Q_{A}(\zeta)\right|^{2} d \mu(\zeta) \leqslant\|A\|^{2}
$$

Proof. The first identity is an easy calculation, and the inequalities follow by computing the eigenvalues of the quadratic form $\sum \lambda_{i}^{2}+\left(\sum \lambda_{i}\right)^{2}-c \lambda_{i_{0}}^{2}, c>0$. The lower bound is attained e.g. for $Q_{A}(\zeta)=\left|\zeta_{1}\right|^{2}-\frac{1}{n}\left(\left|\zeta_{2}\right|^{2}+\ldots+\left|\zeta_{n}\right|^{2}\right)$ when we take $i_{0}=1$ and $c=1+\frac{1}{n}$.

Proof of the Probabilistic estimate 2.19. Take a vector $\zeta \in T_{X, z}, \zeta=\sum \zeta_{i} \frac{\partial}{\partial z_{i}}$, with $\|\zeta\|_{\omega}=1$, and introduce the trace free sesquilinear quadratic form

$$
Q_{z, \zeta}(u)=\sum_{i, j, \alpha, \beta} \widetilde{c}_{i j \alpha \beta}(z) \zeta_{i} \bar{\zeta}_{j} u_{\alpha} \bar{u}_{\beta}, \quad \widetilde{c}_{i j \alpha \beta}=c_{i j \alpha \beta}-\frac{1}{r} \eta_{i j} \delta_{\alpha \beta}, \quad u \in \mathbb{C}^{r}
$$

where $\eta_{i j}=\sum_{1 \leqslant \alpha \leqslant r} c_{i j \alpha \alpha}$. We consider the corresponding trace free curvature tensor

$$
\widetilde{\Theta}_{V}=\frac{i}{2 \pi} \sum_{i, j, \alpha, \beta} \widetilde{c}_{i j \alpha \beta} d z_{i} \wedge d \bar{z}_{j} \otimes e_{\alpha}^{*} \otimes e_{\beta}
$$

As a general matter of notation, we adopt here the convention that the canonical correspondence between Hermitian forms and (1,1)-forms is normalized as $\sum a_{i j} d z_{i} \otimes d \bar{z}_{j} \leftrightarrow$ $\frac{i}{2 \pi} \sum a_{i j} d z_{i} \wedge d \bar{z}_{j}$, and we take the liberty of using the same symbols for both types of objects; we do so especially for $g_{k}(z, x, u)$ and $\eta(z)=\frac{i}{2 \pi} \sum \eta_{i j}(z) d z_{i} \wedge d \bar{z}_{j}=\operatorname{Tr} \Theta_{V}(z)$. First observe that for all $k$-tuples of unit vectors $u=\left(u_{1}, \ldots, u_{k}\right) \in\left(S^{2 r-1}\right)^{k}, u_{s}=\left(u_{s \alpha}\right)_{1 \leqslant \alpha \leqslant r}$, we have

$$
\int_{\left(S^{2 r-1}\right)^{k}}\left|\sum_{1 \leqslant s \leqslant k} \frac{1}{s} x_{s} \sum_{i, j, \alpha, \beta} \widetilde{c}_{i j \alpha \beta}(z) \zeta_{i} \bar{\zeta}_{j} u_{s \alpha} \bar{u}_{s \beta}\right|^{2} d \mu(u)=\sum_{1 \leqslant s \leqslant k} \frac{x_{s}^{2}}{s^{2}} \mathbf{V}\left(Q_{z, \zeta}\right)
$$

where $\mathbf{V}\left(Q_{z, \zeta}\right)$ is the variance of $Q_{z, \zeta}$ on $S^{2 r-1}$. This is so because we have a sum over $s$ of independent random variables on $\left(S^{2 r-1}\right)^{k}$, all of which have zero mean value (Lemma 2.26 shows that the variance $\mathbf{V}(Q)$ of a trace free Hermitian quadratic form 
$Q(u)=\sum_{1 \leqslant \alpha \leqslant r} \lambda_{\alpha}\left|u_{\alpha}\right|^{2}$ on the unit sphere $S^{2 r-1}$ is equal to $\frac{1}{r(r+1)} \sum \lambda_{\alpha}^{2}$, but we only give the formula to fix the ideas). Formula (2.22) yields

$$
\int_{\Delta_{k-1}} x_{s}^{2} d \nu_{k, r}(x)=\frac{r+1}{k(k r+1)} .
$$

Therefore, according to notation (2.15), we obtain the partial variance formula

$$
\begin{aligned}
\int_{\Delta_{k-1} \times\left(S^{2 r-1}\right)^{k}} \mid g_{k}(z, x, u)(\zeta) & -\left.\bar{g}_{k}(z, x)(\zeta)\right|^{2} d \nu_{k, r}(x) d \mu(u) \\
& =\frac{(r+1)}{k(k r+1)}\left(\sum_{1 \leqslant s \leqslant k} \frac{1}{s^{2}}\right) \sigma_{h}\left(\widetilde{\Theta}_{V}(\zeta, \zeta)\right)^{2}
\end{aligned}
$$

in which

$$
\begin{aligned}
& \bar{g}_{k}(z, x)(\zeta)=\sum_{1 \leqslant s \leqslant k} \frac{1}{s} x_{s} \frac{1}{r} \sum_{i j \alpha} c_{i j \alpha \alpha} \zeta_{i} \bar{\zeta}_{j}=\left(\sum_{1 \leqslant s \leqslant k} \frac{1}{s} x_{s}\right) \frac{1}{r} \eta(z)(\zeta), \\
& \sigma_{h}\left(\widetilde{\Theta}_{V}(\zeta, \zeta)\right)^{2}=\mathbf{V}\left(u \mapsto\left\langle\widetilde{\Theta}_{V}(\zeta, \zeta) u, u\right\rangle_{h}\right)=\int_{u \in S^{2 r-1}}\left|\left\langle\widetilde{\Theta}_{V}(\zeta, \zeta) u, u\right\rangle_{h}\right|^{2} d \mu(u)
\end{aligned}
$$

By integrating over $\zeta \in S^{2 n-1} \subset \mathbb{C}^{n}$ and applying the left hand inequality in Lemma 2.26 we infer

$$
\begin{array}{rl}
\int_{\Delta_{k-1} \times\left(S^{2 r-1}\right)^{k}}\left\|g_{k}(z, x, u)-\bar{g}_{k}(z, x)\right\|_{\omega}^{2} & d \nu_{k, r}(x) d \mu(u) \\
& \leqslant \frac{n^{2}(r+1)}{k(k r+1)}\left(\sum_{1 \leqslant s \leqslant k} \frac{1}{s^{2}}\right) \sigma_{\omega, h}\left(\widetilde{\Theta}_{V}\right)^{2}
\end{array}
$$

where $\sigma_{\omega, h}\left(\widetilde{\Theta}_{V}\right)$ is the standard deviation of $\widetilde{\Theta}_{V}$ on $S^{2 n-1} \times S^{2 r-1}$ :

$$
\sigma_{\omega, h}\left(\widetilde{\Theta}_{V}\right)^{2}=\int_{|\zeta|_{\omega}=1,|u|_{h}=1}\left|\left\langle\widetilde{\Theta}_{V}(\zeta, \zeta) u, u\right\rangle_{h}\right|^{2} d \mu(\zeta) d \mu(u) .
$$

On the other hand, brutal estimates give the Hermitian operator norm estimates

$$
\begin{aligned}
\left\|\bar{g}_{k}(z, x)\right\|_{\omega} & \leqslant\left(\sum_{1 \leqslant s \leqslant k} \frac{1}{s} x_{s}\right) \frac{1}{r}\|\eta(z)\|_{\omega}, \\
\left\|g_{k}(z, x, u)\right\|_{\omega} & \leqslant\left(\sum_{1 \leqslant s \leqslant k} \frac{1}{s} x_{s}\right)\left\|\Theta_{V}\right\|_{\omega, h}
\end{aligned}
$$

where

$$
\left\|\Theta_{V}\right\|_{\omega, h}=\sup _{|\zeta|_{\omega}=1,|u|_{h}=1}\left|\left\langle\Theta_{V}(\zeta, \zeta) u, u\right\rangle_{h}\right| .
$$

We use these estimates to evaluate the $q$-index integrals. The integral associated with $\bar{g}_{k}(z, x)$ is much easier to deal with than $g_{k}(z, x, u)$ since the characteristic function of the $q$-index set depends only on $z$. By Lemma 2.25 we find

$$
\begin{aligned}
& \left|\mathbb{1}_{g_{k}, q}(z, x, u) \operatorname{det} g_{k}(z, x, u)-\mathbb{1}_{\eta, q}(z) \operatorname{det} \bar{g}_{k}(z, x)\right| \\
& \quad \leqslant\left\|g_{k}(z, x, u)-\bar{g}_{k}(z, x)\right\|_{\omega} \sum_{0 \leqslant i \leqslant n-1}\left\|g_{k}(z, x, u)\right\|_{\omega}^{i}\left\|\bar{g}_{k}(z, x)\right\|_{\omega}^{n-1-i} .
\end{aligned}
$$


The Cauchy-Schwarz inequality combined with $(2.28-2.30)$ implies

$$
\begin{aligned}
& \int_{\Delta_{k-1} \times\left(S^{2 r-1}\right)^{k}}\left|\mathbb{1}_{g_{k}, q}(z, x, u) \operatorname{det} g_{k}(z, x, u)-\mathbb{1}_{\eta, q}(z) \operatorname{det} \bar{g}_{k}(z, x)\right| d \nu_{k, r}(x) d \mu(u) \\
& \leqslant\left(\int_{\Delta_{k-1} \times\left(S^{2 r-1}\right)^{k}}\left\|g_{k}(z, x, u)-\bar{g}_{k}(z, x)\right\|_{\omega}^{2} d \nu_{k, r}(x) d \mu(u)\right)^{1 / 2} \times \\
&\left(\int_{\Delta_{k-1} \times\left(S^{2 r-1}\right)^{k}}\left(\sum_{0 \leqslant i \leqslant n-1}\left\|g_{k}(z, x, u)\right\|_{\omega}^{i}\left\|\bar{g}_{k}(z, x)\right\|_{\omega}^{n-1-i}\right)^{2} d \nu_{k, r}(x) d \mu(u)\right)^{1 / 2} \\
& \leqslant \frac{n(1+1 / r)^{1 / 2}}{(k(k+1 / r))^{1 / 2}}\left(\sum_{1 \leqslant s \leqslant k} \frac{1}{s^{2}}\right)^{1 / 2} \sigma_{\omega, h}\left(\widetilde{\Theta}_{V}\right) \sum_{1 \leqslant i \leqslant n-1}\left\|\Theta_{V}\right\|_{\omega, h}^{i}\left(\frac{1}{r}\|\eta(z)\|_{\omega}\right)^{n-1-i} \\
& \times\left(\int_{\Delta_{k-1}}\left(\sum_{1 \leqslant s \leqslant k} \frac{x_{s}}{s}\right)^{2 n-2} d \nu_{k, r}(x)\right)^{1 / 2}=O\left(\frac{(\log k)^{n-1}}{k^{n}}\right)
\end{aligned}
$$

by Lemma 2.20 with $n$ replaced by $2 n-2$. This is the essential error estimate. As one can see, the growth of the error mainly depends on the final integral factor, since the initial multiplicative factor is uniformly bounded over $X$. In order to get the principal term, we compute

$$
\begin{aligned}
\int_{\Delta_{k-1}} \operatorname{det} \bar{g}_{k}(z, x) d \nu_{k, r}(x) & =\frac{1}{r^{n}} \operatorname{det} \eta(z) \int_{\Delta_{k-1}}\left(\sum_{1 \leqslant s \leqslant k} \frac{x_{s}}{s}\right)^{n} d \nu_{k, r}(x) \\
& \sim \frac{(\log k)^{n}}{r^{n} k^{n}} \operatorname{det} \eta(z) .
\end{aligned}
$$

From there we conclude that

$$
\begin{aligned}
\int_{z \in X} \int_{(x, u) \in \Delta_{k-1} \times\left(S^{2 r-1}\right)^{k}} \mathbb{1}_{g_{k}, q}(z, x, u) & g_{k}(z, x, u)^{n} d \nu_{k, r}(x) d \mu(u) \\
& =\frac{(\log k)^{n}}{r^{n} k^{n}} \int_{X} \mathbb{1}_{\eta, q} \eta^{n}+O\left(\frac{(\log k)^{n-1}}{k^{n}}\right)
\end{aligned}
$$

The probabilistic estimate 2.19 follows by $(2.17)$.

(2.31) Remark. If we take care of the precise bounds obtained above, the proof gives in fact the explicit estimate

$$
\int_{X_{k}^{\mathrm{GG}}\left(L_{k}, q\right)} \Theta_{L_{k}, \Psi_{h, p, \varepsilon}^{*}}^{n+k r-1}=\frac{(n+k r-1) ! I_{k, r, n}}{n !(k !)^{r}(k r-1) !}\left(\int_{X} \mathbb{1}_{\eta, q} \eta^{n}+\varepsilon_{k, r, n} J\right)
$$

where

$$
J=n(1+1 / r)^{1 / 2}\left(\sum_{s=1}^{k} \frac{1}{s^{2}}\right)^{1 / 2} \int_{X} \sigma_{\omega, h}\left(\widetilde{\Theta}_{V}\right) \sum_{i=1}^{n-1} r^{i+1}\left\|\Theta_{V}\right\|_{\omega, h}^{i}\|\eta(z)\|_{\omega}^{n-1-i} \omega^{n}
$$


and

$$
\begin{aligned}
\left|\varepsilon_{k, r, n}\right| \leqslant & \frac{\left(\int_{\Delta_{k-1}}\left(\sum_{s=1}^{k} \frac{x_{s}}{s}\right)^{2 n-2} d \nu_{k, r}(x)\right)^{1 / 2}}{(k(k+1 / r))^{1 / 2} \int_{\Delta_{k-1}}\left(\sum_{s=1}^{k} \frac{x_{s}}{s}\right)^{n} d \nu_{k, r}(x)} \\
\leqslant & \frac{\left(1+\frac{1}{3} \sum_{m=2}^{2 n-2} \frac{2^{m}(2 n-2) !}{(2 n-2-m) !}\left(1+\frac{1}{2}+\ldots+\frac{1}{k}\right)^{-m}\right)^{1 / 2}}{1+\frac{1}{2}+\ldots+\frac{1}{k}} \sim \frac{1}{\log k}
\end{aligned}
$$

by the lower and upper bounds of $I_{k, r, n}, I_{k, r, 2 n-2}$ obtained in Lemma 2.20. As $(2 n-2) ! /(2 n-2-m) ! \leqslant(2 n-2)^{m}$, one easily shows that

$$
\left|\varepsilon_{k, r, n}\right| \leqslant \frac{(31 / 15)^{1 / 2}}{\log k} \quad \text { for } k \geqslant e^{5 n-5} .
$$

Also, we see that the error terms vanish if $\widetilde{\Theta}_{V}$ is identically zero, but this is of course a rather unexpected circumstance. In general, since the form $\widetilde{\Theta}_{V}$ is trace free, Lemma 2.23 applied to the quadratic form $u \mapsto\left\langle\widetilde{\Theta}_{V}(\zeta, \zeta) u, u\right\rangle$ on $\mathbb{C}^{r}$ implies

$$
\sigma_{\omega, h}\left(\widetilde{\Theta}_{V}\right) \leqslant(r+1)^{-1 / 2}\left\|\widetilde{\Theta}_{V}\right\|_{\omega, h}
$$

This yields the simpler bound

$$
J \leqslant n r^{1 / 2}\left(\sum_{s=1}^{k} \frac{1}{s^{2}}\right)^{1 / 2} \int_{X}\left\|\widetilde{\Theta}_{V}\right\|_{\omega, h} \sum_{i=1}^{n-1} r^{i}\left\|\Theta_{V}\right\|_{\omega, h}^{i}\|\eta(z)\|_{\omega}^{n-1-i} \omega^{n} .
$$

It will be useful to extend the above estimates to the case of sections of

$$
L_{k}=\mathscr{\sigma}_{X_{k}^{\mathrm{GG}}}(1) \otimes \pi_{k}^{*} \mathscr{\odot}\left(\frac{1}{k r}\left(1+\frac{1}{2}+\ldots+\frac{1}{k}\right) F\right)
$$

where $F \in \operatorname{Pic}_{\mathbb{Q}}(X)$ is an arbitrary $\mathbb{Q}$-line bundle on $X$ and $\pi_{k}: X_{k}^{\mathrm{GG}} \rightarrow X$ is the natural projection. We assume here that $F$ is also equipped with a smooth Hermitian metric $h_{F}$. In formulas (2.17-2.19), the renormalized curvature $\eta_{k}(z, x, u)$ of $L_{k}$ takes the form

$$
\eta_{k}(z, x, u)=\frac{1}{\frac{1}{k r}\left(1+\frac{1}{2}+\ldots+\frac{1}{k}\right)} g_{k}(z, x, u)+\Theta_{F, h_{F}}(z),
$$

and by the same calculations its expected value is

$$
\eta(z):=\mathbf{E}\left(\eta_{k}(z, \bullet, \bullet)\right)=\Theta_{\operatorname{det} V^{*}, \operatorname{det} h^{*}}(z)+\Theta_{F, h_{F}}(z)
$$

Then the variance estimate for $\eta_{k}-\eta$ is unchanged, and the $L^{p}$ bounds for $\eta_{k}$ are still valid, since our forms are just shifted by adding the constant smooth term $\Theta_{F, h_{F}}(z)$. The probabilistic estimate 2.18 is therefore still true in exactly the same form, provided 
we use $(2.34-2.36)$ instead of the previously defined $L_{k}, \eta_{k}$ and $\eta$. An application of holomorphic Morse inequalities gives the desired cohomology estimates for

$$
\begin{aligned}
h^{q}\left(X, E_{k, m}^{\mathrm{GG}} V^{*}\right. & \left.\otimes \odot\left(\frac{m}{k r}\left(1+\frac{1}{2}+\ldots+\frac{1}{k}\right) F\right)\right) \\
& =h^{q}\left(X_{k}^{\mathrm{GG}}, \sigma_{X_{k}^{\mathrm{GG}}}(m) \otimes \pi_{k}^{*} \odot\left(\frac{m}{k r}\left(1+\frac{1}{2}+\ldots+\frac{1}{k}\right) F\right)\right),
\end{aligned}
$$

provided $m$ is sufficiently divisible to give a multiple of $F$ which is a $\mathbb{Z}$-line bundle.

(2.37) Theorem. Let $(X, V)$ be a directed manifold, $F \rightarrow X$ a $\mathbb{Q}$-line bundle, $(V, h)$ and $\left(F, h_{F}\right)$ smooth Hermitian structure on $V$ and $F$ respectively. We define

$$
\begin{aligned}
& L_{k}=\mathscr{G}_{X_{k}^{\mathrm{GG}}}(1) \otimes \pi_{k}^{*} \mathscr{O}\left(\frac{1}{k r}\left(1+\frac{1}{2}+\ldots+\frac{1}{k}\right) F\right), \\
& \eta=\Theta_{\operatorname{det} V^{*}, \operatorname{det} h^{*}}+\Theta_{F, h_{F}} \text {. }
\end{aligned}
$$

Then for all $q \geqslant 0$ and all $m \gg k \gg 1$ such that $m$ is sufficiently divisible, we have

$$
\begin{aligned}
h^{q}\left(X_{k}^{\mathrm{GG}}, \mathscr{O}\left(L_{k}^{\otimes m}\right)\right) \leqslant \frac{m^{n+k r-1}}{(n+k r-1) !} \frac{(\log k)^{n}}{n !(k !)^{r}}\left(\int_{X(\eta, q)}(-1)^{q} \eta^{n}+O\left((\log k)^{-1}\right)\right) \\
h^{0}\left(X_{k}^{\mathrm{GG}}, \mathscr{O}\left(L_{k}^{\otimes m}\right)\right) \geqslant \frac{m^{n+k r-1}}{(n+k r-1) !} \frac{(\log k)^{n}}{n !(k !)^{r}}\left(\int_{X(\eta, \leqslant 1)} \eta^{n}-O\left((\log k)^{-1}\right)\right) \\
\chi\left(X_{k}^{\mathrm{GG}}, \mathscr{O}\left(L_{k}^{\otimes m}\right)\right)=\frac{m^{n+k r-1}}{(n+k r-1) !} \frac{(\log k)^{n}}{n !(k !)^{r}}\left(c_{1}\left(V^{*} \otimes F\right)^{n}+O\left((\log k)^{-1}\right)\right) .
\end{aligned}
$$

Green and Griffiths [GG79] already checked the Riemann-Roch calculation $(2.37 \mathrm{c})$ in the special case $V=T_{X}^{*}$ and $F=\mathscr{\sigma}_{X}$. Their proof is much simpler since it relies only on Chern class calculations, but it cannot provide any information on the individual cohomology groups, except in very special cases where vanishing theorems can be applied; in fact in dimension 2, the Euler characteristic satisfies $\chi=h^{0}-h^{1}+h^{2} \leqslant h^{0}+h^{2}$, hence it is enough to get the vanishing of the top cohomology group $H^{2}$ to infer $h^{0} \geqslant \chi$; this works for surfaces by means of a well-known vanishing theorem of Bogomolov which implies in general

$$
\left.H^{n}\left(X, E_{k, m}^{\mathrm{GG}} T_{X}^{*} \otimes \odot\left(\frac{m}{k r}\left(1+\frac{1}{2}+\ldots+\frac{1}{k}\right) F\right)\right)\right)=0
$$

as soon as $K_{X} \otimes F$ is big and $m \gg 1$.

In fact, thanks to Bonavero's singular holomorphic Morse inequalities [Bon93], everything works almost unchanged in the case where $V \subset T_{X}$ has singularities and $h$ is an admissible metric on $V$ (see (0.8)). We only have to find a blow-up $\mu: \widetilde{X}_{k} \rightarrow X_{k}$ so that the resulting pull-backs $\mu^{*} L_{k}$ and $\mu^{*} V$ are locally free, and $\mu^{*} \operatorname{det} h^{*}, \mu^{*} \Psi_{h, p, \varepsilon}$ only have divisorial singularities. Then $\eta$ is a $(1,1)$-current with logarithmic poles, and we have to deal with smooth metrics on $\mu^{*} L_{k}^{\otimes m} \otimes \mathscr{O}\left(-m E_{k}\right)$ where $E_{k}$ is a certain effective divisor on 
$X_{k}$ (which, by our assumption (0.8), does not project onto $X$ ). The cohomology groups involved are then the twisted cohomology groups

$$
H^{q}\left(X_{k}^{\mathrm{GG}}, \mathscr{O}\left(L_{k}^{\otimes m}\right) \otimes \mathscr{F}_{k, m}\right)
$$

where $\mathscr{f}_{k, m}=\mu_{*}\left(\mathscr{O}\left(-m E_{k}\right)\right)$ is the corresponding multiplier ideal sheaf, and the Morse integrals need only be evaluated in the complement of the poles, that is on $X(\eta, q) \backslash S$ where $S=\operatorname{Sing}(V) \cup \operatorname{Sing}(h)$. Since

$$
\left.\left(\pi_{k}\right)_{*}\left(\circlearrowleft\left(L_{k}^{\otimes m}\right) \otimes \mathscr{J}_{k, m}\right) \subset E_{k, m}^{\mathrm{GG}} V^{*} \otimes \odot\left(\frac{m}{k r}\left(1+\frac{1}{2}+\ldots+\frac{1}{k}\right) F\right)\right)
$$

we still get a lower bound for the $H^{0}$ of the latter sheaf (or for the $H^{0}$ of the un-twisted line bundle $\mathcal{G}\left(L_{k}^{\otimes m}\right)$ on $\left.X_{k}^{\mathrm{GG}}\right)$. If we assume that $K_{V} \otimes F$ is big, these considerations also allow us to obtain a strong estimate in terms of the volume, by using an approximate Zariski decomposition on a suitable blow-up of $(X, V)$. The following corollary implies in particular Theorem 0.5 .

(2.38) Corollary. If $F$ is an arbitrary $\mathbb{Q}$-line bundle over $X$, one has

$$
\begin{aligned}
& h^{0}\left(X_{k}^{\mathrm{GG}}, \mathscr{\sigma}_{X_{k}^{\mathrm{GG}}}(m) \otimes \pi_{k}^{*} \mathscr{G}\left(\frac{m}{k r}\left(1+\frac{1}{2}+\ldots+\frac{1}{k}\right) F\right)\right) \\
& \geqslant \frac{m^{n+k r-1}}{(n+k r-1) !} \frac{(\log k)^{n}}{n !(k !)^{r}}\left(\operatorname{Vol}\left(K_{V} \otimes F\right)-O\left((\log k)^{-1}\right)\right)-o\left(m^{n+k r-1}\right),
\end{aligned}
$$

when $m \gg k \gg 1$, in particular there are many sections of the $k$-jet differentials of degree $m$ twisted by the appropriate power of $F$ if $K_{V} \otimes F$ is big.

Proof. The volume is computed here as usual, i.e. after performing a suitable modification $\mu: \widetilde{X} \rightarrow X$ which converts $K_{V}$ into an invertible sheaf. There is of course nothing to prove if $K_{V} \otimes F$ is not big, so we can assume $\operatorname{Vol}\left(K_{V} \otimes F\right)>0$. Let us fix smooth Hermitian metrics $h_{0}$ on $T_{X}$ and $h_{F}$ on $F$. They induce a metric $\mu^{*}\left(\operatorname{det} h_{0}^{-1} \otimes h_{F}\right)$ on $\mu^{*}\left(K_{V} \otimes F\right)$ which, by our definition of $K_{V}$, is a smooth metric. By the result of Fujita [Fuj94] on approximate Zariski decomposition, for every $\delta>0$, one can find a modification $\mu_{\delta}: \widetilde{X}_{\delta} \rightarrow X$ dominating $\mu$ such that

$$
\mu_{\delta}^{*}\left(K_{V} \otimes F\right)=\sigma_{\widetilde{X}_{\delta}}(A+E)
$$

where $A$ and $E$ are $\mathbb{Q}$-divisors, $A$ ample and $E$ effective, with

$$
\operatorname{Vol}(A)=A^{n} \geqslant \operatorname{Vol}\left(K_{V} \otimes F\right)-\delta
$$

If we take a smooth metric $h_{A}$ with positive definite curvature form $\Theta_{A, h_{A}}$, then we get a singular Hermitian metric $h_{A} h_{E}$ on $\mu_{\delta}^{*}\left(K_{V} \otimes F\right)$ with poles along $E$, i.e. the quotient $h_{A} h_{E} / \mu^{*}\left(\operatorname{det}_{\widetilde{X}} h_{0}^{-1} \otimes h_{F}\right)$ is of the form $e^{-\varphi}$ where $\varphi$ is quasi-psh with log poles log $\left|\sigma_{E}\right|^{2}$ $\left(\bmod C^{\infty}\left(\widetilde{X}_{\delta}\right)\right)$ precisely given by the divisor $E$. We then only need to take the singular metric $h$ on $T_{X}$ defined by

$$
h=h_{0} e^{\frac{1}{r}\left(\mu_{\delta}\right)^{*} \varphi}
$$


(the choice of the factor $\frac{1}{r}$ is there to correct adequately the metric on $\operatorname{det} V$ ). By construction $h$ induces an admissible metric on $V$ and the resulting curvature current $\eta=\Theta_{K_{V}, \operatorname{det} h^{*}}+\Theta_{F, h_{F}}$ is such that

$$
\mu_{\delta}^{*} \eta=\Theta_{A, h_{A}}+[E], \quad[E]=\text { current of integration on } E .
$$

Then the 0-index Morse integral in the complement of the poles is given by

$$
\int_{X(\eta, 0) \backslash S} \eta^{n}=\int_{\widetilde{X}_{\delta}} \Theta_{A, h_{A}}^{n}=A^{n} \geqslant \operatorname{Vol}\left(K_{V} \otimes F\right)-\delta
$$

and (2.38) follows from the fact that $\delta$ can be taken arbitrary small.

(2.39) Example. In some simple cases, the above estimates can lead to very explicit results. Take for instance $X$ to be a smooth complete intersection of multidegree $\left(d_{1}, d_{2}, \ldots, d_{s}\right)$ in $\mathbb{P}_{\mathbb{C}}^{n+s}$ and consider the absolute case $V=T_{X}$. Then

$$
K_{X}=\mathscr{O}_{X}\left(d_{1}+\ldots+d_{s}-n-s-1\right)
$$

Assume that $X$ is of general type, i.e. $\sum d_{j}>n+s+1$. Let us equip $V=T_{X}$ with the restriction of the Fubini-Study metric $h=\Theta_{\mathscr{O}(1)}$; a better choice might be the KählerEinstein metric but we want to keep the calculations as elementary as possible. The standard formula for the curvature tensor of a submanifold gives

$$
\Theta_{T_{X}, h}=\left(\Theta_{T_{\mathbb{P} n+s}, h}\right)_{\mid X}+\beta^{*} \wedge \beta
$$

where $\beta \in C^{\infty}\left(\Lambda^{1,0} T_{X}^{*} \otimes \operatorname{Hom}\left(T_{X}, \bigoplus \mathcal{O}\left(d_{j}\right)\right)\right)$ is the second fundamental form. In other words, by the well known formula for the curvature of projective space, we have

$$
\left\langle\Theta_{T_{X}, h}(\zeta, \zeta) u, u\right\rangle=|\zeta|^{2}|u|^{2}+|\langle\zeta, u\rangle|^{2}-|\beta(\zeta) \cdot u|^{2}
$$

The curvature $\rho$ of $\left(K_{X}\right.$, det $\left.h^{*}\right)$ (i.e. the opposite of the Ricci form $\left.\operatorname{Tr} \Theta_{T_{X}, h}\right)$ is given by

$$
\rho=-\operatorname{Tr} \Theta_{T_{X}, h}=\operatorname{Tr}\left(\beta \wedge \beta^{*}\right)-(n+1) h \geqslant-(n+1) h
$$

We take here $F=\mathscr{G}_{X}(-a), a \in \mathbb{Q}_{+}$, and we want to determine conditions for the existence of sections

$$
H^{0}\left(X, E_{k, m}^{\mathrm{GG}} T_{X}^{*} \otimes \odot\left(-a \frac{m}{k r}\left(1+\frac{1}{2}+\ldots+\frac{1}{k}\right)\right)\right), \quad m \gg 1 .
$$

We have to choose $K_{X} \otimes \mathscr{\sigma}_{X}(-a)$ ample, i.e. $\sum d_{j}>n+s+a+1$, and then (by an appropriate choice of the metric of $\left.F=\mathscr{O}_{X}(-a)\right)$, the form $\eta=\Theta_{K_{X} \otimes \mathscr{O}_{X}(-a)}$ can be taken to be any positive form cohomologous to $\left(\sum d_{j}-(n+s+a+1)\right) h$. We use remark 2.31 and estimate the error terms by considering the Kähler metric

$$
\omega=\rho+(n+s+2) h \equiv\left(\sum d_{j}+1\right) h
$$


Inequality (2.40) shows that $\omega \geqslant 2 h$ and also that $\omega \geqslant \operatorname{Tr}\left(\beta \wedge \beta^{*}\right)$. From this, one easily concludes that $\|\eta\|_{\omega} \leqslant 1$ by an appropriate choice of $\eta$, as well as $\left\|\Theta_{T_{X}, h}\right\|_{\omega, h} \leqslant 1$ and $\left\|\widetilde{\Theta}_{T_{X}, h}\right\|_{\omega, h} \leqslant 2$. By (2.33), we obtain for $n \geqslant 2$

$$
J \leqslant n^{3 / 2} \frac{\pi}{\sqrt{6}} \times 2 \frac{n^{n}-1}{n-1} \int_{X} \omega^{n}<\frac{4 \pi}{\sqrt{6}} n^{n+1 / 2} \int_{X} \omega^{n}
$$

where $\int_{X} \omega^{n}=\left(\sum d_{j}+1\right)^{n} \operatorname{deg}(X)$. On the other hand, the leading term $\int_{X} \eta^{n}$ equals $\left(\sum d_{j}-n-s-a-1\right)^{n} \operatorname{deg}(X)$ with $\operatorname{deg}(X)=d_{1} \ldots d_{s}$. By the bound (2.32) on the error term $\varepsilon_{k, r, n}$, we find that the leading coefficient of the growth of our spaces of sections is strictly controlled below by a multiple of

$$
\left(\sum d_{j}-n-s-a-1\right)^{n}-4 \pi\left(\frac{31}{90}\right)^{1 / 2} \frac{n^{n+1 / 2}}{\log k}\left(\sum d_{j}+1\right)^{n}
$$

if $k \geqslant e^{5 n-5}$. A sufficient condition for the existence of sections in (2.41) is thus

$$
k \geqslant \exp \left(7.38 n^{n+1 / 2}\left(\frac{\sum d_{j}+1}{\sum d_{j}-n-s-a-1}\right)^{n}\right) .
$$

This is good in view of the fact that we can cover arbitrary smooth complete intersections of general type. On the other hand, even when the degrees $d_{j}$ tend to $+\infty$, we still get a large lower bound $k \sim \exp \left(7.38 n^{n+1 / 2}\right)$ on the order of jets, and this is far from being optimal: Diverio [Div08, Div09] has shown e.g. that one can take $k=n$ for smooth hypersurfaces of high degree. It is however not unlikely that one could improve estimate (2.42) with more careful choices of $\omega, h$. 


\section{References}

[ABP73] M.F. Atiyah, R. Bott and V.K. Patodi. - On the heat equation and the index theorem, Invent. Math., 19 (1973), 279-330.

[AN54] Y. Akizuki, S. Nakano. - Note on Kodaira-Spencer's proof of Lefschetz theorems, Proc. Jap. Acad., 30 (1954), 266-272.

[AG62] A. Andreotti, H. Grauert. - Théorèmes de finitude pour la cohomologie des espaces complexes, Bull. Soc. Math. France, 90 (1962), 193-259.

[AV65] A. Andreotti, E. Vesentini. - Carleman estimates for the Laplace-Beltrami equation in complex manifolds, Publ. Math. I.H.E.S., 25 (1965), 81-130.

[Ang96] F. Angelini. - An algebraic version of Demailly's asymptotic Morse inequalities, Proc. Amer. Math. Soc., 124 (1996), 3265-3269.

[Ber10] G. Bérczi. - Thom polynomials and the Green-Griffiths conjecture, arXiv:1011.4710, 61p.

[BeKi10] G. Bérczi, F. Kirwan. - A geometric construction for invariant jet differentials, arXiv: 1012.1797, 42p.

[BmD09] R. Berman, J.-P. Demailly. - Regularity of plurisubharmonic upper envelopes in big cohomology classes, arXiv:math.CV/0905.1246v1.

[Blo26] A. Bloch. - Sur les systèmes de fonctions uniformes satisfaisant à l'équation d'une variété algébrique dont l'irrégularité dépasse la dimension, J. de Math, 5 (1926), 19-66.

[Boc48] S. Bochner. - Curvature and Betti numbers (I) and (II), Ann. of Math., 49 (1948), 379-390; 50 (1949), 77-93.

[Bog79] F.A. Bogomolov. - Holomorphic tensors and vector bundles on projective varieties, Math. USSR Izvestija, 13/3 (1979), 499-555.

[Bom70] E. Bombieri. - Algebraic values of meromorphic maps, Invent. Math., 10 (1970), 267-287 and Addendum, Invent. Math., 11 (1970), 163-166.

[Bon93] L. Bonavero. - Inégalités de Morse holomorphes singulières, C. R. Acad. Sci. Paris Sér. I Math., 317 (1993), 1163-1166.

[Bon98] L. Bonavero. - Inégalités de Morse holomorphes singulières, J. Geom. Anal., 8 (1998), 409-425.

[BU00] D. Borthwick, A. Uribe. - Nearly Kählerian embeddings of symplectic manifolds, Asian J. Math., 4 (2000), 599-620.

[Bot57] R. Bott. - Homogeneous vector bundles, Ann. of Math., 66 (1957), 203-248.

[Bou89] Th. Bouche. - Inégalités de Morse pour la d"'-cohomologie sur une variété holomorphe non compacte, Ann. Sci. École Norm. Sup., 22 (1989), 501-513.

[Bou90] Th. Bouche. - Convergence de la métrique de Fubini-Study d'un fibré linéaire positif, Ann. Inst. Fourier (Grenoble), 40 (1990), 117-130.

[Bck02] S. Boucksom. - Cônes positifs des variétés complexes compactes, Thesis, Grenoble 2002.

[BDPP04] S. Boucksom, J.-P. Demailly, M. Păun, Th. Peternell. - The pseudo-effective cone of a compact Kähler manifold and varieties of negative Kodaira dimension, manuscript May 2004, math.AG/0405285.

[Cle86] H. Clemens. - Curves on generic hypersurfaces, Ann. Sci. Éc. Norm. Sup, 19 (1986), 629-636, Erratum: Ann. Sci. Éc. Norm. Sup. 20 (1987) 281.

[CG76] M. Cowen, P. Griffiths. - Holomorphic curves and metrics of negative curvature, J. Analyse Math, 29 (1976), 93-153. 
[CP04] F. Campana, Th. Peternell. - Geometric stability of the cotangent bundle and the universal cover of a projective manifold, arXiv:math.AG/0405093.

[Dem82] J.-P. Demailly. - Estimations $L^{2}$ pour l'opérateur $\bar{\partial}$ d'un fibré vectoriel holomorphe semi-positif au dessus d'une variété kählérienne complète, Ann. Sci. Ec. Norm. Sup., 15 (1982), 457-511.

[Dem85] J.-P. Demailly. — Champs magnétiques et inégalités de Morse pour la d" -cohomologie, Ann. Inst. Fourier (Grenoble), 35 (1985), 189-229.

[Dem90] J.-P. Demailly. - Singular Hermitian metrics on positive line bundles, Proc. Conf. Complex algebraic varieties (Bayreuth, April 2-6, 1990), edited by K. Hulek, T. Peternell, M. Schneider, F. Schreyer, Lecture Notes in Math., Vol. 1507, Springer-Verlag, Berlin, 1992.

[Dem91] J.-P. Demailly. — Holomorphic Morse inequalities, Lectures given at the AMS Summer Institute on Complex Analysis held in Santa Cruz, July 1989, Proceedings of Symposia in Pure Mathematics, Vol. 52, Part 2 (1991), 93-114.

[Dem92] J.-P. Demailly. — Regularization of closed positive currents and Intersection Theory, J. Alg. Geom., 1 (1992), 361-409.

[Dem93] J.-P. Demailly. - A numerical criterion for very ample line bundles, J. Differential Geom., 37 (1993), 323-374.

[Dem94] J.-P. Demailly. - $L^{2}$ vanishing theorems for positive line bundles and adjunction theory, Lecture Notes of the CIME Session "Transcendental methods in Algebraic Geometry", Cetraro, Italy, July 1994, Ed. F. Catanese, C. Ciliberto, Lecture Notes in Math., Vol. 1646, $1-97$.

[Dem95] J.-P. Demailly. - Algebraic criteria for Kobayashi hyperbolic projective varieties and jet differentials, AMS Summer School on Algebraic Geometry, Santa Cruz 1995, Proc. Symposia in Pure Math., ed. by J. Kollár and R. Lazarsfeld, 76p.

[Dem97] J.-P. Demailly. - Variétés hyperboliques et équations différentielles algébriques, Gaz. Math, 73 (juillet 1997), 3-23.

[Dem01] J.-P. Demailly. — Multiplier ideal sheaves and analytic methods in algebraic geometry, Lecture Notes, School on "Vanishing theorems and effective results in Algebraic Geometry, ICTP Trieste, April 2000, Publications of ICTP, 2001.

[Dem10a] J.-P. Demailly. - Holomorphic Morse inequalities and asymptotic cohomology groups: a tribute to Bernhard Riemann, Milan Journal of Mathematics, 78 (2010), 265-277 and arXiv: math.CV/1003.5067.

[Dem10b] J.-P. Demailly. - A converse to the Andreotti-Grauert theorem, Ann. Faculté des Sciences de Toulouse, Volume spécial en l'honneur de Nguyen Thanh Van, 20 (2011), 123135.

[DEG00] J.-P. Demailly, J. El Goul. - Hyperbolicity of generic surfaces of high degree in projective 3-space, Amer. J. Math, 122 (2000), 515-546.

[DEL00] J.-P. Demailly, L. Ein, R. Lazarsfeld. - A subadditivity property of multiplier ideals, Michigan Math. J., 2000, to appear.

[DK01] J.-P. Demailly, J. Kollár. - Semicontinuity of complex singularity exponents and Kähler-Einstein metrics on Fano orbifolds, math.AG/9910118; Ann. Ec. Norm. Sup., 34 (2001), 525-556.

[Div08] S. Diverio. - Differential equations on complex projective hypersurfaces of low dimension, Compos. Math, 144 (2008), 920-932.

[Div09] S. Diverio. - Existence of global invariant jet differentials on projective hypersurfaces of high degree, Math. Ann, 344 (2009), 293-315.

[DMR10] S. Diverio, J. Merker, E. Rousseau. - Effective algebraic degeneracy, Invent. Math, 180 (2010), 161-223.

[DP04] J.-P. Demailly, M. Păun. - Numerical characterization of the Kähler cone of a compact Kähler manifold, arXiv: math.AG/0105176, Annals of Math, 159 (2004), 1247-1274. 
[DPS94] J.-P. Demailly, Th. Peternell, M. Schneider. - Compact complex manifolds with numerically effective tangent bundles, J. Algebraic Geometry, 3 (1994), 295-345.

[DPS01] J.-P. Demailly, Th. Peternell, M. Schneider. — Pseudo-effective line bundles on compact Kähler manifolds, Internat. J. Math, 12 (2001), 689-741.

[DT10] S. Diverio, S. Trapani. - A remark on the codimension of the Green-Griffiths locus of generic projective hypersurfaces of high degree, J. Reine Angew. Math, 649 (2010), 55-61.

[FKL07] T. de Fernex, A. Küronya, R. Lazarsfeld. - Higher cohomology of divisors on a projective variety, Math. Ann., 337 (2007), 443-455.

[Fuj94] T. Fujita. — Approximating Zariski decomposition of big line bundles, Kodai Math. J., 17 (1994), 1-3.

[Get83] E. Getzler. - Pseudodifferential operators on supermanifolds and the Atiyah-Singer index theorem, Comm. Math. Phys., 92 (1983), 167-178.

[Get87] E. Getzler. — Inégalités asymptotiques de Demailly pour les fibrés vectoriels, C. R. Acad. Sci., 304 (1987), 475-478.

[Get89] E. Getzler. - An analogue of Demailly's inequality for strictly pseudoconvex CR manifolds, J. Diff. Geom., 29 (1989), 231-244.

[GR70] H. Grauert, O. Riemenschneider. - Verschwindungssätze für analytische Kohomologiegruppen auf komplexen Räumen, Invent. Math., 11 (1970), 263-292.

[GG79] M. Green, P. Griffiths. - Two applications of algebraic geometry to entire holomorphic mappings, The Chern Symposium 1979, Proc. Internal. Sympos. Berkeley, CA, 1979, Springer-Verlag, New York, 41-74, 1980.

[Gri69] P.A. Griffiths. - Hermitian differential geometry, Chern classes and positive vector bundles, Global Analysis, papers in honor of K. Kodaira, Princeton Univ. Press, Princeton, 1969, 181-251.

[Har70] R. Hartshorne. - Ample subvarieties of algebraic varieties, Lecture Notes in Math., Vol. 156, Springer-Verlag, Berlin (1970).

[HeSU80] Hess H., Schrader R., Uhlenbock D.A.. - Kato's inequality and the spectral distribution of Laplacians on compact Riemannian manifolds, J. Diffential Geom, 15 (1980), $27-38$.

[Hir64] H. Hironaka. - Resolution of singularities of an algebraic variety over a field of characteristic zero, Ann. of Math., 79 (1964), 109-326.

[Hör65] L. Hörmander. - $L^{2}$ estimates and existence theorems for the $\bar{\partial}$ operator, Acta Math., 113 (1965), 89-152.

[Hov79] A.G. Hovanski. — Geometry of convex bodies and algebraic geometry, Uspehi Mat. Nauk, 34 (4) (1979), 160-161.

[Huy99] D. Huybrechts. - Compact Hyperkähler Manifolds: Basic Results, Invent. Math., 135 (1999), 63-113.

[Huy01] D. Huybrechts. - The Projectivity Criterion for Hyperkähler manifolds as a Consequence of the Demailly-Păun Theorem, personal communication, manuscript Köln University, May 2001, 3 p, to appear in Invent. Math.

[Ji89] S. Ji. - Inequality for distortion function of invertible sheaves on abelian varieties, Duke Math. J., 58 (1989), 657-667.

[Kem89] G. Kempf. - Metrics on invertible sheaves on abelian varieties, Topics in algebraic geometry (Guanajuato, 1989), Aportaciones Mat. Notas Investigación, 5, Soc. Mat. Mexicana, México, 1992, 107-108.

[Kod53] K. Kodaira. - On a differential geometric method in the theory of analytic stacks, Proc. Nat. Acad. Sci. USA, 39 (1953), 1268-1273.

[Kod54] K. Kodaira. - On Kähler varieties of restricted type, Ann. of Math., 60 (1954), 28-48.

[Kür06] A. Küronya. - Asymptotic cohomological functions on projective varieties, Amer. J. Math., 128 (2006), 1475-1519. 
[Lae02] L. Laeng. - Estimations spectrales asymptotiques en géométrie hermitienne, Thèse de Doctorat de l'Université de Grenoble I, octobre 2002, http://www-fourier.ujf-grenoble.fr/THESE/ps/laeng.ps.gz and http://tel.archives-ouvertes.fr/tel-00002098/en/.

[Laz04] R. Lazarsfeld. - Positivity in Algebraic Geometry I.-II, Ergebnisse der Mathematik und ihrer Grenzgebiete, Vols. 48-49, Springer Verlag, Berlin, 2004.

[Lel57] P. Lelong. - Intégration sur un ensemble analytique complexe, Bull. Soc. Math. France, 85 (1957), 239-262.

[Lel69] P. Lelong. - Plurisubharmonic functions and positive differential forms, Gordon and Breach, New-York, and Dunod, Paris, 1969.

[Man93] L. Manivel. - Un théorème de prolongement $L^{2}$ de sections holomorphes d'un fibré vectoriel, Math. Zeitschrift, 212 (1993), 107-122.

[McQ98] M. McQuillan. - Diophantine approximation and foliations, Inst. Hautes Études Sci. Publ. Math, 87 (1998), 121-174.

[McQ99] M. McQuillan. - Holomorphic curves on hyperplane sections of 3-folds, Geom. Funct. Anal, 9 (1999), 370-392.

[Mer10] J. Merker. - Algebraic differential equations for entire holomorphic curves in projective hypersurfaces of general type, arXiv:1005.0405, 89 pages.

[Mil63] J. Milnor. - Morse theory, Based on lecture notes by M. Spivak and R. Wells, Annals of Mathematics Studies, No. 51, Princeton University Press, Princeton, N.J., 1963, 153 pp.

[Nad89] A.M. Nadel. - Multiplier ideal sheaves and Kähler-Einstein metrics of positive scalar curvature, Proc. Nat. Acad. Sci. U.S.A., 86 (1989), 7299-7300 and Annals of Math., 132 (1990), 549-596.

[Nak55] S. Nakano. - On complex analytic vector bundles, J. Math. Soc. Japan, 7 (1955), 1-12.

[Ohs88] T. Ohsawa. - On the extension of $L^{2}$ holomorphic functions, II, Publ. RIMS, Kyoto Univ., 24 (1988), 265-275.

[OT87] T. Ohsawa, K. Takegoshi. - On the extension of $L^{2}$ holomorphic functions, Math. Zeitschrift, 195 (1987), 197-204.

[Pop08] D. Popovici. - Regularization of currents with mass control and singular Morse inequalities, J. Differential Geom., 80 (2008), 281-326.

[Ser54] J.-P. Serre. — Fonctions automorphes: quelques majorations dans le cas où X/G est compact, Sém. Cartan (1953-54) 2-1 à 2-9.

[Ser55] J.-P. Serre. — Un théorème de dualité, Comment. Math., 29 (1955), 9-26.

[SZ02] B. Shiffman, S. Zelditch. - Asymptotics of almost holomorphic sections of ample line bundles on symplectic manifolds, J. Reine Angew. Math., 544 (2002), 181-222.

[Sie55] C.L. Siegel. — Meromorphic Funktionen auf kompakten Mannigfaltigkeiten, Nachrichten der Akademie der Wissenschaften in Göttingen, Math.-Phys. Klasse, 4 (1955), 71-77.

[Siu74] Y.T. Siu. - Analyticity of sets associated to Lelong numbers and the extension of closed positive currents, Invent. Math., 27 (1974), 53-156.

[Siu84] Y.T. Siu. - A vanishing theorem for semi-positive line bundles over non-Kähler manifolds, J. Differential Geometry, 19 (1984), 431-452.

[Siu85] Y.T. Siu. - Some recent results in complex manifold theory related to vanishing theorems for the semi-positive case, Proceedings of the Math. Arbeitstagung 1984, Lecture Notes in Mathematics, 1111 (1985), 169-192.

[Siu90] Y.T. Siu. - Calculus inequalities derived from holomorphic Morse inequalities, Math. Annalen, 286 (1990), 549-558.

[Siu93] Y.T. Siu. - An effective Matsusaka big theorem, Ann. Inst. Fourier, 43 (1993), 1387-1405.

[Siu97] Y.T.Siu. - A proof of the general Schwarz lemma using the logarithmic derivative lemma, Communication personnelle, avril 1997. 
[Siu02] Y.T. Siu. - Some recent transcendental techniques in algebraic and complex geometry, Proceedings of the International Congress of Mathematicians, Vol. I (Beijing, 2002), Higher Ed. Press, Beijing, (2002), 439-448.

[Siu04] Y.T. Siu. - Hyperbolicity in complex geometry, The legacy of Niels Henrik Abel, Springer, Berlin, (2004), 543-566.

[SY96a] Y.T. Siu, S.K. Yeung. - Hyperbolicity of the complement of a generic smooth curve of high degree in the complex projective plane, Invent. Math, 124 (1996), 573-618.

[SY96b] Y.T. Siu, S.K. Yeung. — Defects for ample divisors of Abelian varieties, Schwarz lemma and hyperbolic surfaces of low degree, Preprint (preprint, autumn 1996).

[Sko72] H. Skoda. - Sous-ensembles analytiques d'ordre fini ou infini dans $\mathbb{C}^{n}$, Bull. Soc. Math. France, 100 (1972), 353-408.

[Sko75] H. Skoda. - Estimations $L^{2}$ pour l'opérateur $\bar{\partial}$ et applications arithmétiques, Séminaire P. Lelong (Analyse), année 1975/76, Lecture Notes in Math., Springer-Verlag, Berlin, 538 (1977), 314-323.

[Tei79] B. Teissier. - Du théorème de l'index de Hodge aux inégalités isopérimétriques, C. R. Acad. Sc. Paris, sér. A, 288 (29 Janvier 1979), 287-289.

[Tei82] B. Teissier. - Bonnesen-type inequalities in algebraic geometry, Sem. on Diff. Geom., edited by S.T. Yau, 1982, Princeton Univ. Press, 85-105.

[Tia90] G. Tian. - On a set of polarized Khler metrics on algebraic manifolds, J. Differential Geom., 32 (1990)), 99-130.

[Tot10] B. Totaro. - Line bundles with partially vanishing cohomology, July 2010, arXiv: math.AG/1007.3955.

[Tra95] S. Trapani. - Numerical criteria for the positivity of the difference of ample divisors, Math. Zeitschrift, 219 (1995), 387-401.

[Voi96] C. Voisin. - On a conjecture of Clemens on rational curves on hypersurfaces, J. Diff. Geom, 44 (1996), 200-213. Correction: J. Diff. Geom. 49 (1998), 601-611.

[Wit82] E. Witten. - Supersymmetry and Morse theory, J. Diff. Geom., 17 (1982), 661-692. 